\title{
Luminescent Quantum Dots for High Temperature Sensing
}

\author{
A Dissertation \\ Presented to \\ the faculty of the School of Engineering and Applied Science \\ University of Virginia \\ in partial fulfillment \\ of the requirements for the degree \\ Doctor of Philosophy
}

by

Devin Pugh-Thomas

May

2012 


\section{APPROVAL SHEET}

The dissertation

is submitted in partial fulfillment of the requirements

for the degree of

Doctor of Philosophy

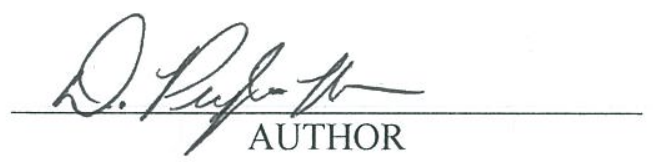

The dissertation has been read and approved by the examining committee:
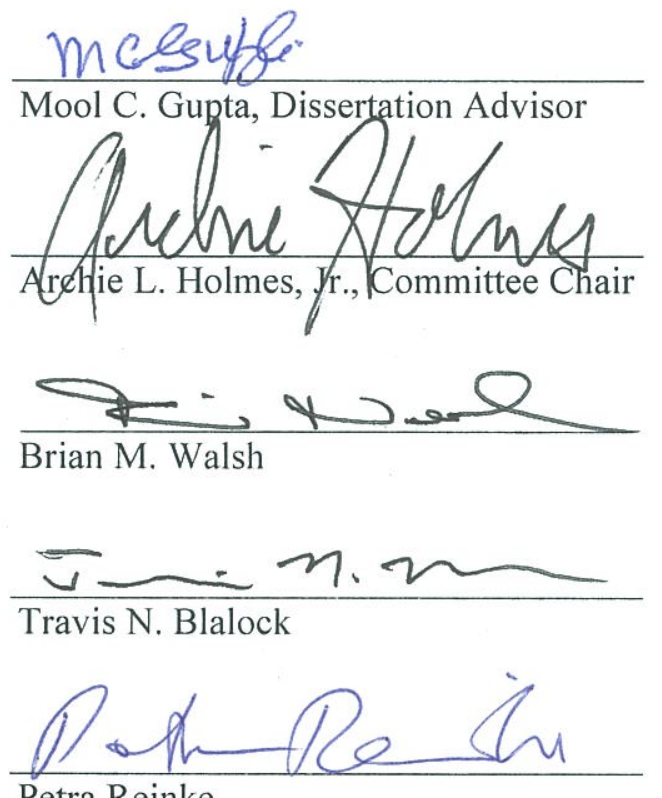

Petra Reinke

Accepted for the School of Engineering and Applied Science:

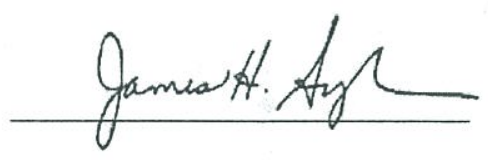

Dean, School of Engineering and Applied Science 


\section{TABLE OF CONTENTS}

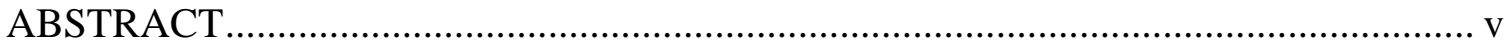

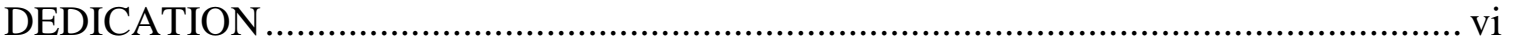

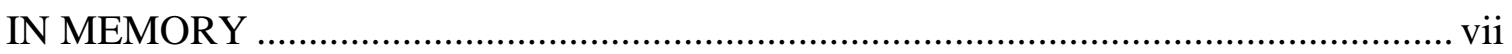

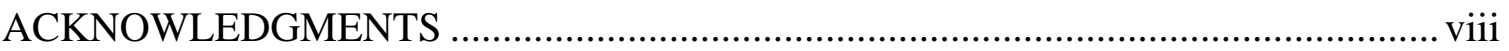

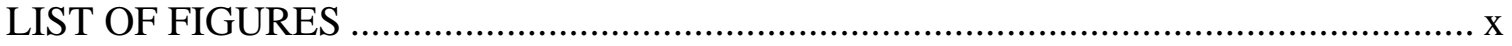

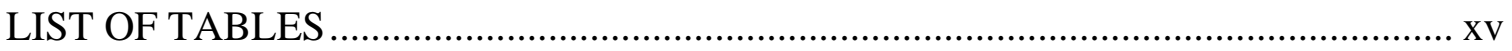

LIST OF ABBREVIATIONS ....................................................................... xvi

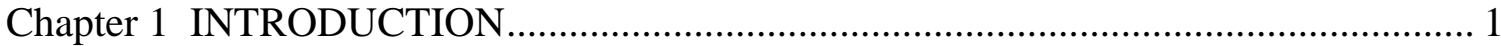

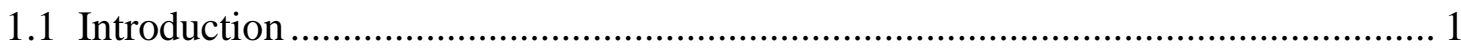

1.2 Background and Relevant Literature Review ................................................. 3

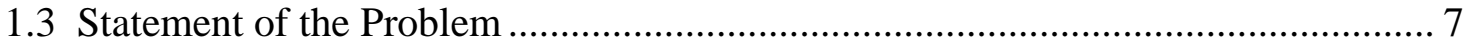

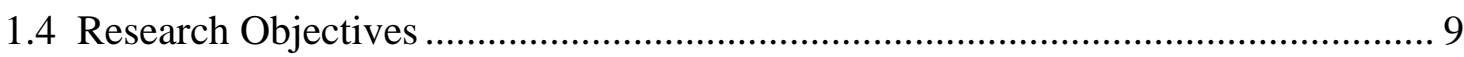

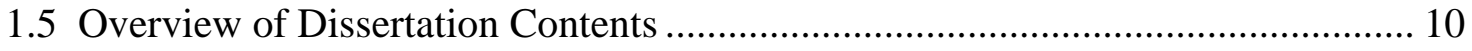

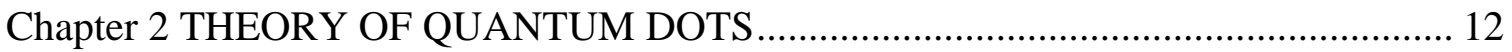

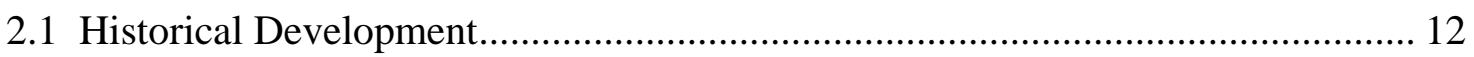

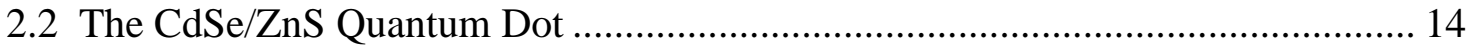

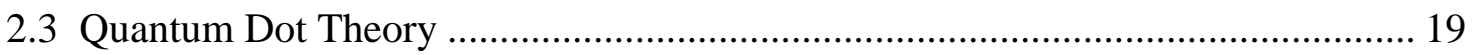

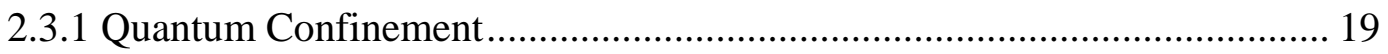

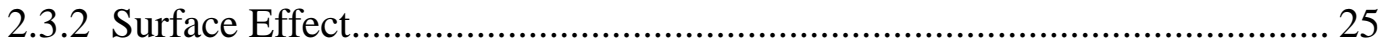

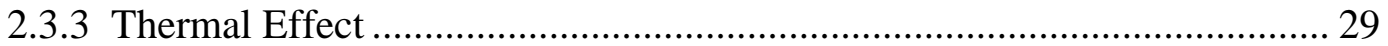

2.4 Quantum Dot Luminescence Thermometry .............................................. 31

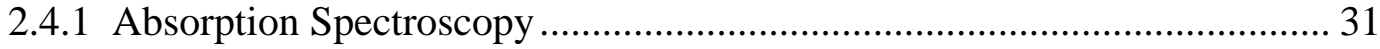

2.4.2 Photoluminescence Spectroscopy .................................................... 34

2.4.3 Time-resolved Photoluminescence Spectroscopy.................................... 37

Chapter 3 QUANTUM DOT THIN FILM FABRICATION AND

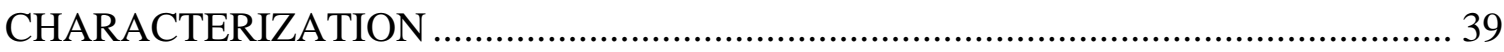

3.1 Sol-gel Preparation of CdSe/ZnS Quantum Dot Thin Films................................ 39

3.2 Characterization using Atomic Force Microscopy (AFM) ................................. 48

Chapter 4 QUANTUM DOT LUMINESCENCE THERMOMETRY ........................... 55 
4.1 Optical Absorption Measurement System........................................................ 55

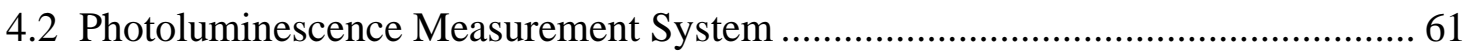

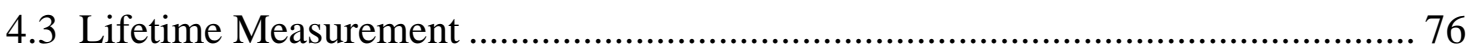

Chapter 5 THERMOMETRY USING QUANTUM DOT CdSe/ZnS:PMMA

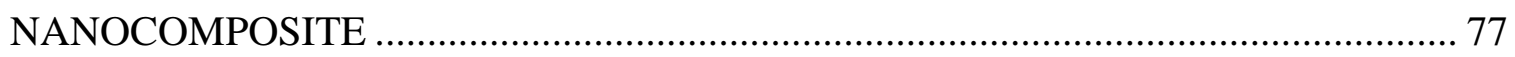

5.1 Temperature Dependent Absorption Spectroscopy........................................... 77

5.2 Temperature Dependent Photoluminescence Spectroscopy …........................... 90

5.3 Temperature Dependent Lifetime Analysis .................................................... 99

Chapter 6 THERMOMETRY USING QUANTUM DOT CdSe/ZnS:SiO 2

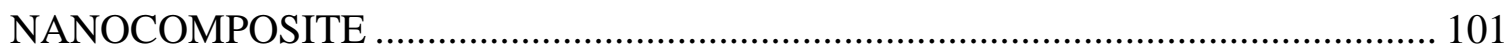

6.1 Temperature Dependent Absorption Spectroscopy ......................................... 101

6.2 Temperature Dependent Photoluminescence Spectroscopy ............................... 114

6.3 Temperature Dependent Lifetime Analysis ................................................ 129

Chapter 7 FIBER OPTIC QD-LUMINESCENCE THERMAL SENSOR .................. 131

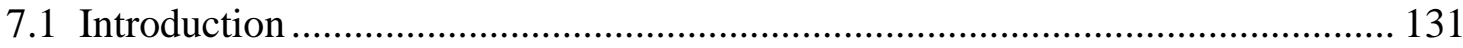

7.2 Fiber Optic Based Quantum Dot Thermal Sensor Design and Fabrication ........ 132

7.3 Fiber Optic Based QD-Thermal Sensor Characterization ................................. 136

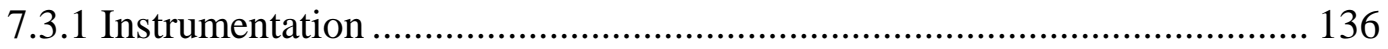

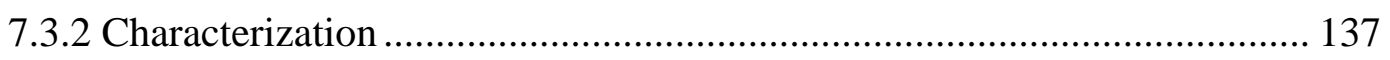

Chapter 8 CONCLUSIONS AND FUTURE WORK ............................................. 142

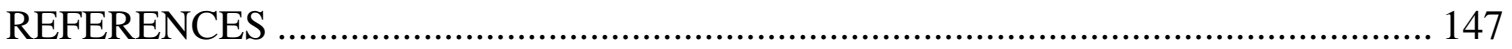

APPENDIX A Advanced Optical Instrumentation for ExplorationVehicles: Orion Le-X

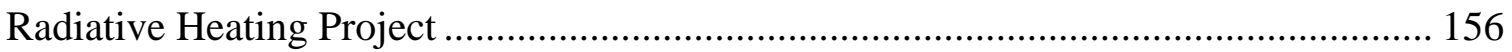

APPENDIX B Research Proposal Submitted to NASA LaRC C\&I ........................... 175

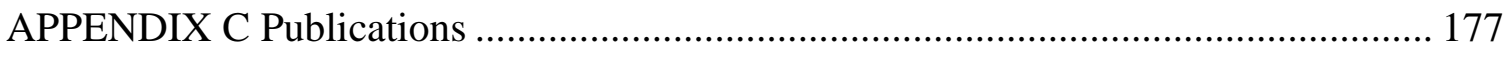

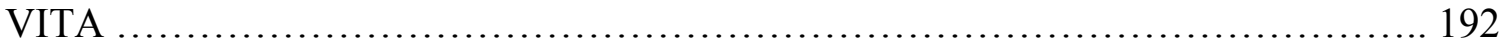




\begin{abstract}
Quantum dots (QDs), semiconductor nanometer sized particles have captured the attention of the scientific community. QDs have unique electrical, optical, and structural properties compared to bulk semiconductors. These properties can be harnessed for optoelectronic device applications. High fluorescent quantum yield, excellent photostability, and narrow-band photoluminescence that can be tuned from the ultraviolet to the near infrared make quantum dots attractive for opto-electronic sensor applications. One of the main factors contributing to the current interest in semiconductor QDs is the need for future miniaturization of electronic and optical devices. For many potential applications of semiconductor quantum dots, the working temperature will be above $295 \mathrm{~K}$. It is therefore of practical interest to study the optical properties of these materials at elevated temperatures.
\end{abstract}

A detailed study of the high temperature ( $\mathrm{T}>295 \mathrm{~K}$ ) characteristics of zinc sulphide encapsulated cadmium selenide quantum dots $(\mathrm{CdSe} / \mathrm{ZnS})$ has been carried out. Optical spectroscopy is used to investigate $\mathrm{CdSe} / \mathrm{ZnS}$ QDs at thermal extremes. The spectral characteristics of $\mathrm{CdSe} / \mathrm{ZnS}$ quantum dots over a range of temperatures is investigated using UV-vis absorption spectroscopy, photoluminescence spectroscopy, and lifetime analysis. The results of this study extend the thermal sensing limit of $\mathrm{CdSe} / \mathrm{ZnS}$ quantum dots and provides framework for sensor development. When embedded into a specialty high temperature matrix, CdSe/ZnS quantum dots exhibit high sensitivity and attractive properties for luminescence thermometry in aerospace applications. Initial work done in collaboration with NASA includes the design, fabrication, and test of specialty aerospace fiber optic sensors. Results pioneered development of a high temperature luminescence-based quantum dot thermal sensor. A fundamental understanding of the temperature dependent optical properties of $\mathrm{CdSe} / \mathrm{ZnS}$ QDs is accomplished. The design, fabrication, and test of a new luminescence-based quantum dot high temperature sensor was carried out. By combining microstructured silica optical fiber with $\mathrm{CdSe} / \mathrm{ZnS}$ QDs, a framework is established that enables investigation of QD properties for application specific fiber optic thermal sensors. 


\section{DEDICATION}

This dissertation is dedicated to my father, Major Isaiah Pugh. 


\section{IN MEMORY}

Mrs. Emma Lou Thomas, "Granny"

Rev. Dr. William J. Marshall 


\section{ACKNOWLEDGMENTS}

"For I know the plans I have for you. Plans to prosper you and not to harm you, plans to give you hope and a future," Jeremiah 29:11. My Lord and Savior Jesus Christ is the strength of my life and my ever-present help at all times.

I thank my husband, Shelley. Thank you for allowing me these years for our future. Thank you for encouraging me to leave comforts and pursue my goals. My heart bursts with appreciation and gratitude for you ... for us.

I thank my mother and sister, Kimberly who have always been there to provide encouragement in the valleys and to celebrate at the peaks.

I express appreciation to my dissertation advisor, Dr. Mool C. Gupta. Thank you for contributing your knowledge and time towards this dissertation. Criticisms, remarks, and advice were kneaded into better self-confidence and an acceptance of independent thought. Thank you for your support.

I thank my technical advisor and mentor, Dr. Brian M. Walsh. Dr. Walsh opened his laboratory at NASA Langley Research Center and gave his time to help me. A fish without water best describes many aspects of my experiences in the UVA-NIA graduate program. Dr. Walsh provided a pond with increasing depth which enabled me to learn, study, and to ultimately define my scientific interests. Thank you for supporting my research interests and laboratory efforts.

I thank Dr. Archie L. Holmes, Dr. Travis N. Blalock, and Dr. Petra Reinke for serving on my dissertation committee.

I thank William C. Edwards and William S. Luck of NASA Langley for supporting my research through equipment support and helpful discussions. I appreciate the many people I've met and worked with at NASA Langley Research Center who prove daily that anything is possible. 
I acknowledge support for my course-work and stipend support from the following: a 1 year National Institute of Aerospace (NIA) Graduate Fellowship and a 2 year NASA Graduate Student Researchers Program (GSRP) fellowship. I am very thankful for these prestigious fellowship opportunities.

The outstanding NASA Langley Student Career Experiences Program, often referred to as the Cooperative (Co-op) Education Program supported my final years of doctoral study and enabled me to complete a life goal. 


\section{LIST OF FIGURES}

Figure 2-1: (a.) CdSe hexagonal wurtzite crystal structure [43]. The gray spheres represent $\mathrm{Cd}$ atoms and the yellow spheres are Se atoms. The sides of the unit cell form a diamond shape. (b.) Extracted from the bold-faced unit cell in (a.), $A_{b}$ is the area of the base, $\mathrm{h}$ is the height of the unit cell, and $\mathrm{V}_{\mathrm{c}}$ is the volume of the unit cell.

Figure 2-2: $\mathrm{CdSe} / \mathrm{ZnS}$ core-shell quantum dot (modified from [45]). Red and blue spheres represent the Cadmium and Selenium atoms. Yellow and green spheres represent the Zinc and Sulphur atoms. 18

Figure 2-3: Cross sectional view of the bottom of the conduction band (CB) and top of the valence band (VB) for (a.) type-I and (b.) type-II QD heterostructures. 18

Figure 2-4: Density of states function versus energy plot for 3-D bulk (blue), 2-D quantum well (red), 1-D quantum wire (green), and 0-D quantum dot (black) structures[48].

Figure 2-5: Schematic of the band structure of a bulk semiconductor (left) and a quantum dot (right).

Figure 2-6: Absorption versus wavelength for bare $\mathrm{CdSe}$ (dashed lines) and $\mathrm{CdSe}(\mathrm{ZnS})$ (solid lines) a $2.3 \mathrm{~nm}, \mathbf{b} 4.2 \mathrm{~nm}, \mathbf{c} 4.8 \mathrm{~nm}$, and d $5.5 \mathrm{~nm}$ quantum dots. Measurements were taken at room temperature [61].

Figure 2-7: Photoluminescence versus wavelength for $4.2 \mathrm{~nm} \mathrm{CdSe} / \mathrm{ZnS}$ dots with a 0 , b 0.65 , c1.3, d 2.6, and e 5.3 monolayer $\mathrm{ZnS}$ coverage. Inset is the Q.Y. versus monolayers of $\mathrm{ZnS}$ shell material. Measurements were taken at room temperature [61] ................... 28

Figure 2-8: Configurational coordinate diagram for $\mathrm{CdSe}$ quantum dots. The blue arrows indicate (1), (2) direct interband transitions, (3) direct intra-subband transitions, and (4) transitions from quantum-confined states to continuum states.

Figure 2-9: Jablonski energy level diagram showing the photoluminescence processes [8].

Figure 3-1: Preparation of $\mathrm{CdSe} / \mathrm{ZnS}: \mathrm{SiO}_{2}$ thin films by spin coating process. 41

Figure 3-2: Calibration curve generated for spin-coating $\mathrm{CdSe} / \mathrm{ZnS}: \mathrm{SiO}_{2} \mathrm{QD}$ films..... 42 
Figure 3-3: (a.) Partially submerged QDs at air-liquid interface and liquid meniscus. The curvature of each local meniscus creates a pressure such that as the droplet dries, the meniscus curvature decreases setting up a pressure gradient which acts as a suction force.

(b.) Outer edge QD rings form due to capillary flow induced by pinned edges 45

Figure 3-5: Preparation of $\mathrm{CdSe} / \mathrm{ZnS}: \mathrm{SiO}_{2}$ films by drop-coating process. 47

Figure 3-6: AFM. The inset is a magnified view of the QD sample mounted on the stage. A Laser diode B Head C Tip D Sample Stage E Mode Selector Switch F Motor Control Switch G Base H Digital Display 50

Figure 3-7: A 20 × $20 \mu \mathrm{m}$ perspective view (top image) and top view (bottom image) of $\mathrm{CdSe} / \mathrm{ZnS}: \mathrm{SiO}_{2}$ film topography. AFM line scans $\mathbf{A}, \mathbf{B}$, and $\mathbf{C}$ are plotted in the following figure.

Figure 3-8: Line profile measurements of AFM image. The regions scanned to generate Height Profile [A], [B], and [C] are shown in Figure 3-7 ..................................... 52

Figure 3-9: (a.) $\mathrm{CdSe} / \mathrm{ZnS}: \mathrm{SiO}_{2} \mathrm{AFM}$ image and (b.) height profile of individual QDs.

Figure 3-10: QD size distribution obtained from several AFM images. ....................... 54

Figure 4-1: Absorption spectroscopy workstation.................................................. 56

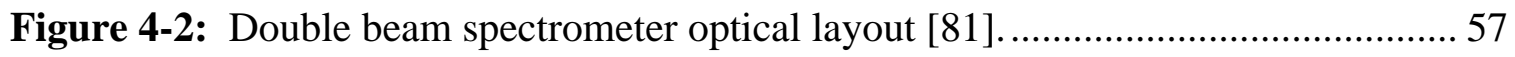

Figure 4-3: Quantum dot thin films mounted in the spectrometer sample chamber..... 59

Figure 4-4: Temperature controller, sample mount, heater, and ceramic apertures....... 60

Figure 4-5: Quantum Dot Luminescence Thermometry Laboratory. A Sample Mount B Optical Fiber C Laser D Collection Lens and Optical Chopper E Spectrometer F PMT G Lock-in Amplifier H Power Supply I Computer

Figure 4-6: Schematic of the measurement system for quantum dot luminescence thermometry 64

Figure 4-7: SPEX 500-M monochromator calibration plot. 66 
Figure 4-8: (a.) Heater cell surrounds the heating element with leads to the temperature controller shown and (b.) A schematic of the aluminum nitride (AIN) heater and macor stage located inside the heater cell.

Figure 4-9: Microheater system electrical flow diagram.

Figure 4-10: Copper terminal leads secure the secondary wires to the terminal blocks. Round-base lug screws hold the braided wires in place. The terminal blocks are screwed onto a macor base plate....

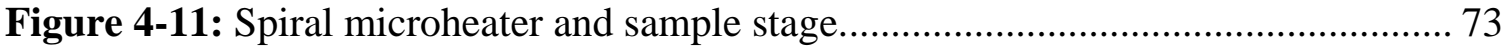

Figure 4-12: \% Power output versus current from the B-PAN control unit. 74

Figure 4-13: Thermocouple temperature reading versus B-PAN control unit temperature.

Figure 5-1: (a.) Room temperature CdSe/ZnS:PMMA nanocomposite absorption spectrum. (b.) Energy level diagram of the optical absorption transitions in CdSe/ZnS quantum dots. The energy values were extracted from (a.)....

Figure 5-3: Absorption spectra of the CdSe/ZnS:PMMA quantum dot at different temperatures.

Figure 5-4: Variation in the first exciton absorption peak wavelength with temperature for $\mathrm{CdSe} / \mathrm{ZnS}$ quantum dots in PMMA.

Figure 5-5: Variation in the full width at half maximum (FWHM) with temperature for $\mathrm{CdSe} / \mathrm{ZnS}$ quantum dots in PMMA.

Figure 5-6: The peak absorption ratio, $1 \mathrm{~S}_{\mathrm{e}} \rightarrow 1 \mathrm{~S}_{3 / 2} / 1 \mathrm{~S}_{\mathrm{e}} \rightarrow 2 \mathrm{~S}_{3 / 2}$ versus temperature. 86

Figure 5-7: Absorption cross section at $\mathrm{T}=295 \mathrm{~K}$ and $\mathrm{T}=325 \mathrm{~K}$ for a $\mathrm{CdSe} / \mathrm{ZnS}: \mathrm{PMMA}$ nanocomposite thin film.

Figure 5-7: Absorption and photoluminescence emission at $295 \mathrm{~K}$ for CdSe/ZnS:PMMA nanocomposite film.

Figure 5-8: $\mathrm{CdSe} / \mathrm{ZnS}$ : PMMA photoluminescence emission measured as a function of temperature. The red arrow in the figure inset represents the CdSe/ZnS QD energy band gap. 
Figure 5-9: Dependence of photoluminescence peak intensity with respect to temperature for CdSe/ZnS:PMMA film. 93

Figure 5-10: PL peak wavelength (circles) versus temperature. Experimental energy band gap for the QD sample (squares) and best fit curve (line).

Figure 5-11: Dependence of the CdSe/ZnS:PMMA full width at half maximum (FWHM) with respect to temperature.

Figure 6-1: Room temperature $\mathrm{CdSe} / \mathrm{ZnS}: \mathrm{CH}_{3} \mathrm{OH}$ nanocomposite solution absorption spectra for Sample A, Sample B, Sample C, and Sample D. 102

Figure 6-2: Absorption spectrum of $\mathrm{CdSe} / \mathrm{ZnS}: \mathrm{SiO}_{2}$ at $\mathrm{T}=295 \mathrm{~K}$ The yellow dashed line is the absorption spectrum and the solid colored lines represent the fit. 104

Figure 6-3: Bulk CdSe sample absorption spectra [93]. 105

Figure 6-4: Absorption coefficient and absorption cross section for a $50 \mu \mathrm{m}$ $\mathrm{CdSe} / \mathrm{ZnS}: \mathrm{SiO}_{2}$ nanocomposite film 108

Figure 6-5: Absorption cross section versus temperature at the $1 \mathrm{~S}_{3 / 2} \rightarrow 1 \mathrm{~S}_{\mathrm{e}}$. 109

Figure 6-6: Temperature dependent absorption spectra from 295-550 K. 111

Figure 6-7: Variation in the first absorption peak wavelength with temperature 112

Figure 6-8: Full width at half maximum (FWHM) versus temperature. 113

Figure 6-9: Normalized photoluminescence emission and absorption spectrum at $295 \mathrm{~K}$ for $50 \mu \mathrm{m}$ thick $\mathrm{CdSe} / \mathrm{ZnS}: \mathrm{SiO}_{2}$ nanocomposite film. 115

Figure 6-10: $\mathrm{CdSe} / \mathrm{ZnS}: \mathrm{SiO}_{2} \mathrm{QD}$ energy band diagram. 116

Figure 6-11: (a.) $\mathrm{CdSe} / \mathrm{ZnS}: \mathrm{SiO}_{2}$ photoluminescence emission measured as a function of temperature and (b.) Ultra-high temperature CdSe/ZnS: $\mathrm{SiO}_{2} \mathrm{PL}$ signal from $450-675 \mathrm{~K}$.

Figure 6-12: $\mathrm{CdSe} / \mathrm{ZnS}: \mathrm{SiO}_{2}$ photoluminescence emission peak wavelength measured as a function of temperature.

Figure 6-13: Temperature dependent energy band gap of CdSe/ZnS:SiO ${ }_{2}$ QD film.... 122 
Figure 6-14: Temperature dependent energy band gap of $\mathrm{CdSe} / \mathrm{ZnS}: \mathrm{SiO}_{2} \mathrm{QD}$ film from 423- $625 \mathrm{~K}$.

Figure 6-15: Variation in the full width at half maximum with respect to temperature.126

Figure 6-16: Variation in the PL intensity with respect to temperature. 128

Figure 7-1: Compact $\mathrm{CdSe} / \mathrm{ZnS}: \mathrm{SiO}_{2}$ thermal sensor design.

Figure 7-2: $\mathrm{CdSe} / \mathrm{ZnS}: \mathrm{SiO}_{2}$ end tip coated optical fiber imaged under (a.) 100X, (b.) and (c.) $200 \mathrm{X}$, and (d-f) 500X microscope objective. Images were acquired with the microscope in (a.) transmission mode, (b-e.) transmission + UV mode, and (f.) UV mode. UV-mode means that the fiber was under UV illumination during imaging. 134

Figure 7-3: $\mathrm{CdSe} / \mathrm{ZnS}: \mathrm{SiO}_{2} \mathrm{QD}$ coated optical fiber on optical microscope stage...... 135

Figure 7-4: Experimental setup used to characterize thermal sensor. 136

Figure 7-5: PL signal output for QD-fiber optic sensor from 295- $625 \mathrm{~K}$ 138

Figure 7-6: Peak intensity versus temperature for $\mathrm{CdSe} / \mathrm{ZnS}: \mathrm{SiO}_{2}$ thermal sensor. ..... 139

Figure 7-7: Peak wavelength versus temperature for $\mathrm{CdSe} / \mathrm{ZnS}: \mathrm{SiO}_{2}$ thermal sensor. Heating (circles) and cool down (squares). 140 


\section{LIST OF TABLES}

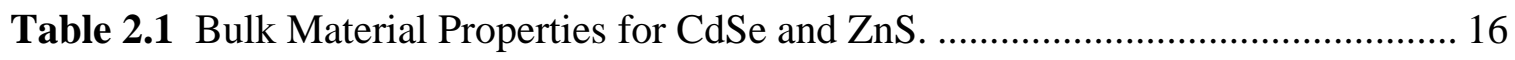

Table 5.1 CdSe(ZnS):PMMA Thermal Sensor Response ....................................... 97

Table 5.2 Oscillator strength and radiative lifetime of $1 \mathrm{~S}_{3 / 2} \rightarrow 1 \mathrm{~S}_{\mathrm{e}}$ first absorption peak.

Table 6.1 Reported colloidal QD optical absorption cross sections.

106

Table 6.2 Optical absorption cross sections for $\mathrm{CdSe} / \mathrm{ZnS}: \mathrm{SiO}_{2}$ quantum dots at different

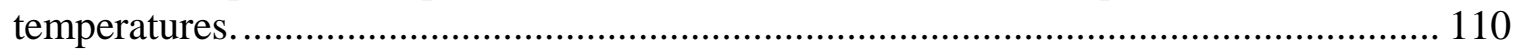

Table 6-3 Comparison of Varshni coefficients for CdSe/ZnS quantum dots. .............. 121

Table 6.4 Oscillator strength and radiative lifetime of $\mathrm{CdSe} / \mathrm{ZnS}: \mathrm{SiO}_{2} \mathrm{QD}$

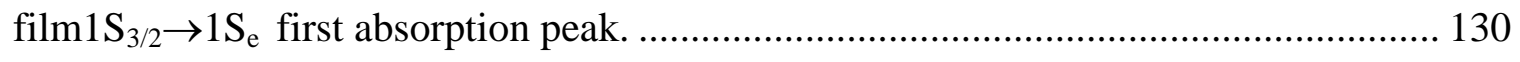

Table 7.1 Comparison of thermal QD sensor to bulk crystal response........................ 141 


\section{LIST OF ABBREVIATIONS}

\section{GENERAL}

$\begin{array}{ll}\text { AFM } & \text { Atomic Force Microscope } \\ \text { CEV } & \text { Crew Exploration Vehicle } \\ \text { EMA } & \text { Effective Mass Model } \\ \text { FWHM } & \text { Full Width at Half Maximum } \\ \text { NASA } & \text { National Aeronautics and Space Administration } \\ \text { NPs } & \text { Nanoparticles } \\ \text { NWs } & \text { Nanowires } \\ \text { PL } & \text { Pholuminescence } \\ \text { PMT } & \text { Photomultiplier Tube } \\ \text { QDs } & \text { Quantum Dots } \\ \text { Sol } & \text { Solution } \\ \text { SOG } & \text { Spin on Glass } \\ \text { T.C. } & \text { Thermocouple } \\ \text { TPS } & \text { Thermal Protection System } \\ & \end{array}$

\section{CHEMICAL}

$\begin{array}{ll}\mathrm{AlN} & \text { Aluminum Nitride } \\ \mathrm{Ar} & \text { Argon } \\ \mathrm{BeAl}_{2} \mathrm{O}_{4}: \mathrm{Cr}^{3+} & \text { Alexandrite } \\ \mathrm{CH}_{3} \mathrm{OH} & \text { Methanol } \\ \mathrm{CdSe} & \text { Cadmium Selenide } \\ \mathrm{CdSe} / \mathrm{ZnS} & \text { Cadmium Selenide/Zinc Sulphide } \\ \mathrm{PMMA} & \text { Poly (methyl methacrylate) } \\ \mathrm{SiO}_{2} & \text { Silicon Dioxide } \\ \mathrm{ZnS} & \text { Zinc Sulphide }\end{array}$




\section{Chapter 1 \\ INTRODUCTION}

"I would like to describe a field in which little has been done, but in which an enormous amount can be done in principle. Furthermore, ... it would have an enormous number of technical applications."

Richard P. Feynman

\subsection{Introduction}

Nanotechnology can be defined as the design and creation of new man-made materials, devices, and functional systems at the nanometer scale. Nanoscience involves the fundamental study of phenomena associated with atomic and molecular interactions in structures sized between $1-100 \mathrm{~nm}$ in at least one dimension. Nanotechnology is the meeting ground of engineering, physics, chemistry, biology, and medicine. In 1959, Feynman postulated on how decreasing the size of things such as shrinking the volumes of an encyclopedia onto the head of a pin and how diagnosing disease through the ability to "swallow the surgeon" would tell us much of great interest about strange phenomena that occur in complex situations at the atomic-scale [1]. Nearly fifteen years later, Norio Taniguchi would coin the term nanotechnology [2]. Today the field is sought to be the primary driver of the $21^{\text {st }}$ century and the new economy. The promise of nanostructures, devices, and systems with at least one novel characteristic is projected to have strong implications in computing, renewable energy, health care, opto-electronics, microelectronics, and sensing. 
Quantum dots (QDs) are nanomaterials with a localized volume termed a dot. These tiny crystalline nanoparticles (NPs) have excitons that are confined in three spatial dimensions. Quantum dots are typically semiconductor nanocrystallites $\sim 2-10 \mathrm{~nm}$ in diameter. Their size is smaller than the materials' bulk-exciton Bohr radius. QDs have distinctly different and remarkably novel optical and electronic properties compared to bulk materials. Quantum dots are readily described as a cluster of atoms because they bridge the gap between small molecules and large crystals. The quantum dot is distinguished from all other nanoparticles by their characteristics of size and composition-dependent optical and electronic properties. Semiconductor quantum dots and the applications derived from them are still in the early stages of technical development. The field is new; with less than 30 years since the discovery of quantum dots. Although the field is growing worldwide, much work still needs to be done in fundamental science towards the ultimate realization of on-the-market practical devices. The expected breakthroughs lie in medical imaging \& diagnosis, real-time sensing, and light emitting diodes. QDs have potential for new anti-counterfeiting technologies and numerous other defense applications. New exploration pathways in aeronautics and aerospace are sought using quantum dots. Namely, the National Aeronautics and Space Administration's (NASA) next-generation technologies include quantum dot lasers for the eye-safe 1.4-2.3 $\mu \mathrm{m}$ range, quantum dot-based photovoltaics, and quantum dot-based opto-electronic sensors [3]. Opto-electronics QD-based sensors for aircraft crack detection, radiation exposure monitoring, and chemical sensing are of interest to NASA. QD-based sensors have potential to monitor instrument heating and material degradation 
under thermal extremes. The potential impact of quantum dots for high temperature measurement motivated this dissertation research.

\subsection{Background and Relevant Literature Review}

Traditional temperature measurement instruments consist of liquid-in glass thermometers, thermocouples, resistance thermometers, and radiation thermometers. As matured technologies, the major sources of their instability, drift, and error are well documented [4]. These instruments find use in many areas because they are readily available off the shelf. However, there exists other optical sensing methods that may exceed the applicability of conventional instruments for application specific thermal sensing. One such method is luminescence thermometry.

Luminescence thermometry is a versatile optical technique for the measurement of temperature. It exploits temperature-dependent changes in the photoluminescent properties of an emissive compound to deduce the temperature of the local environment. The idea of using luminescent materials for temperature measurement was first cited in early 1937 [5]. Luminescence thermometry was first demonstrated in the 1950s when Bradley obtained temperature distributions on a flat phosphor-coated wedge in a supersonic flow field [6]. In another early investigation, a thin sheet of phosphor was mounted onto an aircraft wing in a wind tunnel to image aerodynamic heating [7]. Over the past two decades, luminescence thermometry has leveraged advances in solid state physics, optical fiber technology, and now nanotechnology. Since the luminescencebased fiber-optic sensor market greatly relies on the development of technologies gained from laser development, research has been rooted in rare earth (RE) [8-10]doped optical 
crystals and phosphors [11]. Allison and Gilles measured lifetime decay in yttrium oxide $\left(\mathrm{Y}_{2} \mathrm{O}_{3}\right)$ doped with europium $\left(\mathrm{Y}_{2} \mathrm{O}_{3}: \mathrm{Eu}\right)$, europium doped yttrium vanadate $\left(\mathrm{YVO}_{4}: \mathrm{Eu}\right)$, YAG:Dy and YAG:Tm powders at $1000^{\circ} \mathrm{C}$ [11]. However, no reports have been found on the incorporation of these ultra-high temperature luminescent materials, termed "thermographic phosphors" into practical sensor devices. The published ultra-high temperature luminescent thermometry works have been single-study lifetime decay demonstrations. Chromium-doped phosphors, such as ruby $\left(\mathrm{Al}_{2} \mathrm{O}_{3}: \mathrm{Cr}^{3+}\right)$, spinel $\left(\mathrm{MgAl}_{2} \mathrm{O}_{4}: \mathrm{Cr}^{3+}\right)$, and yttrium aluminum garnet $\left(\mathrm{YAG}: \mathrm{Cr}^{3+}\right)$ have been studied due to relatively long PL lifetimes, chemical, and thermal stability [12-14]. The optical absorption and PL from $\mathrm{Cr}^{3+}$ ions are strongly affected by the host materials because energy levels of d-electrons of $\mathrm{Cr}^{3+}$ ions vary greatly with the crystal field strength and symmetry of oxygen coordination.

Luminescence thermometry has shown potential for temperature measurement in extraordinary conditions. A host of glass and crystalline samples doped with rare-earth ions or transition metal ions have been used as optically active centers for luminescence thermometry [15-17]. Doped fiber and fiber preforms have been fabricated by groups for fiber optic luminescence thermometry [13, 18-21] . Investigations show that temperature sensing is limited by the properties of the material and experimental considerations. Phosphors and bulk crystals have grain sizes on the order of microns. For non-contact thermometry, the phosphors are mixed with the binder material and coated onto the surface of a part of which the temperature is to be measured. The phosphor grain size limits resolution by scattering the excitation light and the emitted light. Thick coatings pose a disadvantage to accurate temperature measurement because 
it acts as an insulating layer, yielding temperature measurement results that cannot be applied to similar uncoated parts. In theory, quantum dots exhibit potential to rival traditionally used rare-earth ions, transition metal ions, and traditional luminescent dyes as optically active centers for thermal sensing.

According to Rayleigh's approximation, the intensity of light scattered from isotropic particles is proportional to the sixth power of the particle diameter [22]. Hence, a $100 \mathrm{~nm}$ particle will scatter one million times more than a $10 \mathrm{~nm}$ particle such as a quantum dot. Compared to the scattering in traditional micrometer-sized particles, the scattering in nanoparticles is near zero. This suggests that a significantly higher spatial resolution is possible by using quantum dot nanoparticles for fluorescence thermometry. Quantum dots theoretically have higher quantum efficiency than conventional bulk crystals and phosphors. The oscillator strength is an important optical parameter that determines the absorption cross-section, recombination rate, and the radiative lifetime. The oscillator strength of an exciton is:

$$
f=\frac{2 m}{\hbar} \Delta E|\mu|^{2}|U(0)|^{2}
$$

where, $\mathrm{m}$ is the electron mass, $\hbar$ is the reduced Planck's constant, $\Delta \mathrm{E}$ is the transition energy, $\mu$ is the transition dipole moment, and $U(0)$ denotes the overlap factor. The overlap factor is the probability of finding an electron and hole at the same site. On the nanoscale, the overlap factor increases largely due to quantum confinement (reference Chapter 2), thus yielding an increase in oscillator strength. An enhancement of the oscillator strength is expected due to the exciton recombination rate in nanostructure 
materials being larger than bulk. The luminescence efficiency is also proportional to the exciton oscillator strength. Therefore, quantum dots have the potential for enhancement via size quantum confinement.

Recently, temperature dependent photoluminescence (PL) was demonstrated using zinc sulfide encapsulated cadmium selenide (CdSe/ZnS) quantum dots [23, 24] . Walker et al. report the first quantum dot luminescence temperature probe by cooling CdSe/ZnS-PLMA, poly(lauryl methacrylate) thin films from 315-100 K [24]. A thin disk of CdSe/ZnS-PLMA was mounted into a cryostat and excited by a $480 \mathrm{~nm}$ laser beam. The PL emission peak wavelength blue shifts by $20 \mathrm{~nm}$ and exhibits a linear and reversible temperature dependence. CdSe, CdSe/ZnS and CdTe quantum dots with different size and subsequently different emission wavelengths have been applied in various ways to detect temperature in the low range, from 4 to $140 \mathrm{~K}$ and 100 to $300 \mathrm{~K}$ [23-25]. PL thermometry has been reported within a narrow range of ambient temperatures from 283 to $300 \mathrm{~K}$ [26]. Other works have shown that the optical properties of quantum dots are retained in solution and certain polymer matrices. The spectral and temporal modes show a temperature dependence for low $(<295 \mathrm{~K})$ temperature sensing [27-29]. These investigations opened the field for QD-based luminescence thermometry. However, the high temperature response has never been thoroughly investigated. In addition, an extensive literature review reveals that the majority of QD-luminescence thermometry studies have little to no theoretical analysis and limited explanations of observations. 


\subsection{Statement of the Problem}

Heat shield technology is a critical component to manned spaceflight. The Orion Crew Exploration Vehicle (CEV) requires thermal protection system (TPS) beyond the current state of the art. A key challenge in TPS advancement is the ability to diagnose TPS performance and incorporate the data into future designs. New approaches to spacecraft health monitoring require advanced photonics technologies. The NASA Orion Le-X TPS Radiative Heating Project was started to develop advanced optical instrumentation for (1) TPS performance characterization and (2) flight instrument heat monitoring. The project background and goals are presented in Appendix A. Work carried out included optical test coupon fabrication, experimental design, and arc jet tests. The results (reference Appendix A) of the Orion Le-X project show for the first time in the history of NASA the use of specialty fiber optic sensors in an arc jet. The study and work on Le-X aided in the conception, framework, and focus for research carried out in this dissertation.

An opportunity to enhance research on quantum dots for luminescence thermometry was granted through the NASA Langley Research Center (LaRC) Creativity and Innovation (C\&I) Fund. The proposal, written by D. Pugh-Thomas is presented in Appendix B.

In this thesis, Cadmium Selenide/Zinc Sulfide $(\mathrm{CdSe} / \mathrm{ZnS})$ core-shell quantum dots are studied in order to understand the basic physics and to probe new and perhaps unexpected phenomenon that occur in quantum confined structures under thermal extremes. The behavior of quantum dots at elevated temperatures and explanation of 
these processes supports QD-based next generation opto-electronic thermal sensors. Critical questions, both in terms of fundamental science and practical device applications are: (1) how to matrix quantum dots into a media to yield a thermally stable, rigid, and optically transparent QD nanocomposite, (2) how does the QD optical spectrum evolve with temperature, and (3) what is the physics framework that supports the data? Then, an extension of the science towards device application is sought by addressing question (4) how to use the data to demonstrate a fiber optic luminescence-based QD thermal sensor and other applications?

At $295 \mathrm{~K}$, the bulk CdSe absorption spectrum exhibits a featureless continuum above the band gap of the material. In contrast, the room temperature CdSe quantum dot absorption spectrum is highly structured. Extensive work on the comparison of experiments to the theory of quantum-confined electronic states in semiconductor quantum dots have provided a description of the nanocrystal band gap variation with particle size [30-32]. It is well known that the band gap of bulk semiconductors is temperature dependent. For example, the band gap of bulk CdSe is $1.75 \mathrm{eV}$ at $300 \mathrm{~K}$ and $1.85 \mathrm{eV}$ at $0 \mathrm{~K}$. However, as previously discussed, the literature on the quantum dot CdSe energy band gap variation with temperature is slim. During the course of this research, the behavior of semiconductor quantum dots under high temperature has become increasingly more important to the scientific community [33, 34]. For many potential applications of semiconductor quantum dots, the working temperature will be above $295 \mathrm{~K}$.

As this thesis will show, the Cadmium Selenide/ Zinc Sulfide (CdSe/ZnS) coreshell quantum dot is a complex system. A plethora of competing and complimentary 
processes work to make $\mathrm{CdSe} / \mathrm{ZnS}$ quantum dots an attractive material for luminescence thermometry. CdSe/ZnS quantum dots have high damage thresholds which establish them as robust emitters. High PL quantum yield has been demonstrated [35]. Due to their versatile surface chemistry, CdSe/ZnS QDs can be dispersed into various hosts [27]. However, the technology of robust, reproducible QD thin film fabrication that can withstand thermal extremes has yet to be mastered. Charge carrier energy transfer in quantum dots coupled with elevated temperatures has remained unexplored. These topics will be addressed as they arise in the context of this thesis.

\subsection{Research Objectives}

The goal of this research is to investigate $\mathrm{CdSe} / \mathrm{ZnS}$ semiconductor quantum dots for application to thermal sensing. Towards this application, the following objectives are set:

1. To investigate fiber optic sensors for advanced photonics flight instrumentation in collaboration with NASA (reference Appendix A).

2. To investigate bulk-crystal alexandrite as a baseline luminescence thermometry study.

3. To investigate CdSe/ZnS:PMMA nanocomposite optical absorption, photoluminescence, and lifetime properties as a function of temperature.

4. To develop the methodology for $\mathrm{CdSe} / \mathrm{ZnS}: \mathrm{SiO}_{2}$ nanocomposite thin film fabrication. To characterize $\mathrm{CdSe} / \mathrm{ZnS}: \mathrm{SiO}_{2}$ nanocomposite film via atomic force microscopy (AFM).

5. To investigate $\mathrm{CdSe} / \mathrm{ZnS}: \mathrm{SiO}_{2}$ nanocomposite optical absorption, photoluminescence, and lifetime properties as a function of temperature.

6. To demonstrate a $\mathrm{CdSe} / \mathrm{ZnS}: \mathrm{SiO}_{2}$ fiber optic luminescence-based high temperature sensor. 


\subsection{Overview of Dissertation Contents}

Chapter 1 begins with an introduction to nanotechnology and semiconductor quantum dots. The field of luminescence thermometry is introduced and the limitations with the current state of the art in luminescence thermometry using bulk crystals are discussed. QD-based luminescence thermometry research is presented in a literature review. The literature search reveals the potential for new research on quantum dots for thermal sensing. A statement of the problem is brought forward through basic research questions which help shape the direction of this thesis.

Chapter 2 begins with some historical perspective from early investigations on small particles to recent steps toward understanding quantum confined structures. Then the quantum dot material under study in this thesis, Cadmium Selenide/Zinc Sulphide $(\mathrm{CdSe} / \mathrm{ZnS})$ is introduced. The theory of quantum dots is presented. Background on optical spectroscopy and the temperature effect on quantum dots is presented.

In Chapter 3, the CdSe/ZnS core-shell quantum dot as the selected quantum dot material is further defined. The sol-gel fabrication of $\mathrm{CdSe} / \mathrm{ZnS}$ QD thin films is outlined and the experimental techniques are presented. Material characterization was carried out using atomic force microscopy (AFM). AFM results and analysis on the CdSe/ZnS QD nanocomposite films are presented.

Chapter 4 includes experimental apparatus, laboratory procedures, and calibration reports for luminescence thermometry. Luminescence thermometry involves extensive experimental apparatus and technical know-how. Luminescence thermometry experiments were carried out after the design and fabrication of two heating units. The 
details and specifications for carrying out absorption measurements, continuous wave photoluminescence, and pulsed photoluminescence measurements are reported.

Chapter 5 includes the results and analysis of temperature-dependent optical absorption, photoluminescence, and lifetime analysis on CdSe/ZnS:PMMA nanocomposite thin films. The CdSe/ZnS quantum dots are characterized up to the thermal limit of the CdSe/ZnS PMMA nanocomposite.

Chapter 6 includes results and analysis of temperature-dependent optical absorption, photoluminescence, and lifetime analysis on $\mathrm{CdSe} / \mathrm{ZnS}: \mathrm{SiO}_{2}$ thin films are presented. The $\mathrm{CdSe} / \mathrm{ZnS}$ quantum dots are characterized near the thermal limit of the high temperature resistant $\mathrm{SiO}_{2}$ matrix.

In Chapter 7, the results and analysis of a $\mathrm{CdSe} / \mathrm{ZnS}: \mathrm{SiO}_{2}$ QD nanocomposite optical fiber luminescence-based high temperature thermal sensor are presented. A compact sensor design and experimental details are presented. The results are compared against the current standard in luminescence-based thermal sensing.

Chapter 8 summarizes the research and suggestions for future work are outlined. 


\section{Chapter 2 \\ THEORY OF QUANTUM DOTS}

"So, as we go down and fiddle around with the atoms down there, we are working with different laws, . . . expect to do different things."

Richard P. Feynman

This chapter begins with some historical perspective on quantum dots. The $\mathrm{CdSe} / \mathrm{ZnS}$ quantum dot is introduced in Section 2.2. The primary quantum mechanical model used to explain concepts such as energy band gap, exciton Bohr radius, and quantum confinement in low-dimensional structures is presented in Section 2.3. Background on the surface effect and the thermal effect conclude the section. The science of quantum dot luminescence thermometry is presented in Section 2.4.

\subsection{Historical Development}

Although Feynman is credited for having first outlined the possibilities of science and technology at the nanoscale, the usage of nano-sized materials dates back to ancient times. In ancient times metal nanoparticles were formed in molten glass to make stained glass objects. In 1856, Faraday recorded the first experiments on the preparation and physical properties of metal colloids [36]. Colloidal quantum dots emerged from a few research laboratories in the 1980s. The size dependence of the optical properties of semiconductor quantum dots was discovered experimentally in the early 1980s in two different experiments: A. I. Ekimov using doped glass and in colloidal solutions by L. E. 
Brus [37-39]. Ekimov and colleagues at the Ioffe Physical-Technical Institute in St. Petersburg observed unusual optical spectra from samples of heat treated glass containing cadmium sulfide (CdS), cadmium selenide (CdSe) and copper chloride ( $\mathrm{CuCl})$ [37]. Ekimov et al. hypothesized that heat caused the semiconductor crystallites to precipitate in the glass and that confinement of electrons in the crystallites caused the unusual optical behavior. Another first hint into zero-dimensional quantum confinement was made at Bell Labs by Brus and coworkers while investigating CdS crystallites formed in aqueous solution [39]. Brus and Efros [40, 41] demonstrated that the band gap of a semiconductor can shift to larger values when the particle size was decreased. Other field pioneers include A. Henglein, A. P. Alivisatos, V. I. Klimov, and M. G. Bawendi. The mid-1990s yielded the synthesis of the first high quality colloidal quantum dots by Bawendi's research group [42] . Prototype opto-electronic QD-based devices such as bench-top lasers and infra-red photodetectors have been reported within the past decade. The new century has to date been marked by a push for improvements in controlled syntheses. Global efforts are being made in fabrication, processing, and optimization of material properties of quantum dots using two main techniques: epitaxial growth and solution phase growth often referred to as colloidal chemistry. Colloidal means that quantum dots are suspended in a chemical mixture. The focus here is on colloidal dots because colloidal QDs are the type of quantum dots used in this thesis. QD preparation techniques, film fabrication, and materials characterization is brought forth in Chapter 3. 


\subsection{The CdSe/ZnS Quantum Dot}

It is instructive to begin with a brief description of bulk CdSe and then introduce the $\mathrm{CdSe} / \mathrm{ZnS}$ quantum dot. CdSe is a binary semiconductor compound of cadmium and selenide. CdSe falls in the II-VI subcategory of semiconductor compounds due to the position of cadmium and selenium on the periodic table. CdSe is a direct band gap semiconductor. Three crystalline forms of CdSe are known: wurtzite (hexagonal), sphalerite (cubic), and rock salt (cubic). The sphalerite CdSe structure is unstable and converts to the wurtzite form upon moderate $\left(\mathrm{T} \sim 95^{\circ} \mathrm{C}\right)$ heating. The rock-salt structure is only observed under high pressure. The wurtzite (w) structure is stable at high temperatures. The w-structure is a member of the hexagonal crystal system and consists of tetrahedrally coordinated $\mathrm{Cd}$ and Se atoms. The wurtzite structure has a hexagonal unit cell with two lattice parameters, a and c. For a real w-structure, bonds between nearest neighbor anion and cation have two distinct lengths. Along the $\mathrm{c}$ axis, the length of the axial bond is defined as $\mathrm{R}^{(1)}=\mathrm{uc}$. Three other bonds have length defined by: $\mathrm{R}^{(2)}=\mathrm{a}\left[1 / 3+(1 / 2-\mathrm{u})^{2}(\mathrm{c} / \mathrm{a})\right]^{1 / 2}$, where $\mathrm{u}$ is a dimensionless internal cell structure parameter. For an ideal w-structure, $c / a=(8 / 3)^{1 / 2}$ and $u=3 / 8$ such that $R^{(1)}=R^{(2)}$. The internal parameter, $\mathrm{u}$ is defined as the length of the bond parallel to the c-axis (anioncation distance or nearest neighbor distance) divided by the $\mathrm{c}$ lattice parameter. The wCdSe has a multi atom basis. The two element, two atom basis for element 1 is $(0,0,0)$ and element 2 is $(2 / 3,1 / 3,1 / 2)$. Figure $2-1$ is a schematic of the crystal structure. The unit cell, (solid-black lines in Figure 2-1a) contains 4 atoms per unit cell, 2 atoms from element 1 and 2 atoms from element 2. Bulk properties for $\mathrm{CdSe}$ and $\mathrm{ZnS}$ are listed in Table 2.1. Throughout the text the notation CdSe is understood to be w-CdSe. 


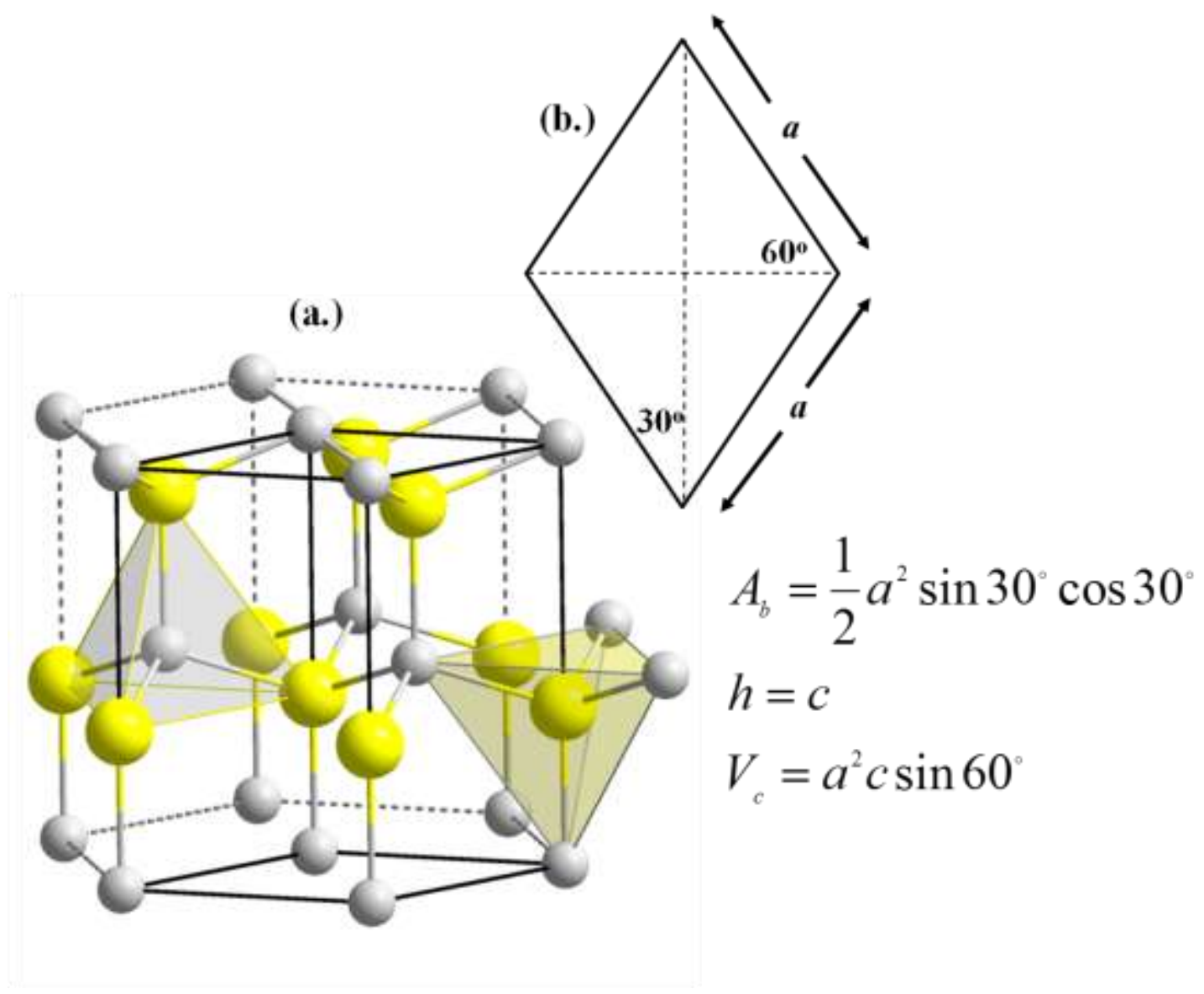

Figure 2-1: (a.) CdSe hexagonal wurtzite crystal structure [43]. The gray spheres represent $\mathrm{Cd}$ atoms and the yellow spheres are Se atoms. The sides of the unit cell form a diamond shape. (b.) Extracted from the bold-faced unit cell in (a.), $A_{b}$ is the area of the base, $h$ is the height of the unit cell, and $V_{c}$ is the volume of the unit cell. 
Table 2.1 Bulk Material Properties for CdSe and $\mathrm{ZnS}$.

\begin{tabular}{|l|c|c|}
\hline Material Property & CdSe & $\mathrm{ZnS}$ \\
\hline \hline Lattice Parameters & $\mathrm{a}=4.2985 \AA, \mathrm{c}=7.0105 \AA$ & $\mathrm{a}=3.811 \AA, \mathrm{c}=6.234 \AA$ \\
\hline Formula Units/ Cell (z) & $\mathrm{z}=2$ & $\mathrm{z}=2$ \\
\hline Density & $5.816 \mathrm{~g} / \mathrm{cm}^{3}$ & $3.98 \mathrm{~g} / \mathrm{cm}^{3}$ \\
\hline Melting Point & $1541 \mathrm{~K}\left(1268^{\circ} \mathrm{C}\right)$ & $1458 \mathrm{~K}\left(1185^{\circ} \mathrm{C}\right)$ \\
\hline Crystal Structure & Hexagonal wurtzite & Hexagonal wurtzite \\
\hline Band gap & $1.74 \mathrm{eV}$ at $300 \mathrm{~K}$ & $3.91 \mathrm{eV}$ at $300 \mathrm{~K}$ \\
\hline Refractive index & 2.5 & $2.356,2.378$ \\
\hline
\end{tabular}

\begin{tabular}{|c||c|c|}
\hline \hline Element & Atomic Weight $(\mathrm{g} / \mathrm{mol})$ & $\begin{array}{c}\text { Covalent Radius }(\AA) \\
{[44]}\end{array}$ \\
\hline \hline $\mathrm{Cd}$ & 112.411 & 1.44 \\
\hline $\mathrm{Se}$ & 78.96 & 1.20 \\
\hline $\mathrm{Zn}$ & 65.38 & 1.22 \\
\hline $\mathrm{S}$ & 32.065 & 1.05 \\
\hline
\end{tabular}


Figure 2-2 is a schematic of a colloidal $\mathrm{CdSe} / \mathrm{ZnS}$ quantum dot heterostructure. The quantum dot is composed of a semiconductor nanocrystal called the core and a thin layer of a different semiconductor nanocrystal called the shell. Cadmium Selenide $(\mathrm{CdSe})$ is the core material. Zinc Sulfide $(\mathrm{ZnS})$ is the shell material. An inorganic outer layer of molecules called ligands (not shown in Figure 2-2) surrounds the core. Ligands are functional groups that enable QDs to be soluble in various solvents. Ligands typically have surface trap sites that act as non-radiative de-excitation channels. Overcoating the core with a layer of a second semiconductor with higher energy band gap is a technique used to improve QD-core stability and enhance photoluminescence quantum yield [22]. The enhancement in PL quantum yield is due in part to band alignment. The CdSe/ZnS quantum dot has type-I band alignment.

The electron-hole (e- h) pair photogenerated in the core remains in the confined spatial region of the core. This type of confinement describes type-I band alignment. Figure 2-3 illustrates the two common QD heteropair band alignments. By definition, in type-I heteropairs, the band gap of one material lies inside of the band gap of another material. Limited mobility of electron (e)- hole (h) to the same spatial region allows for enhanced radiative decay. Therefore, type-I are more suitable than type-II structures for luminescence-based thermal sensing. Type-II band alignment separates e - $\mathrm{h}$ into different spatial regions. 


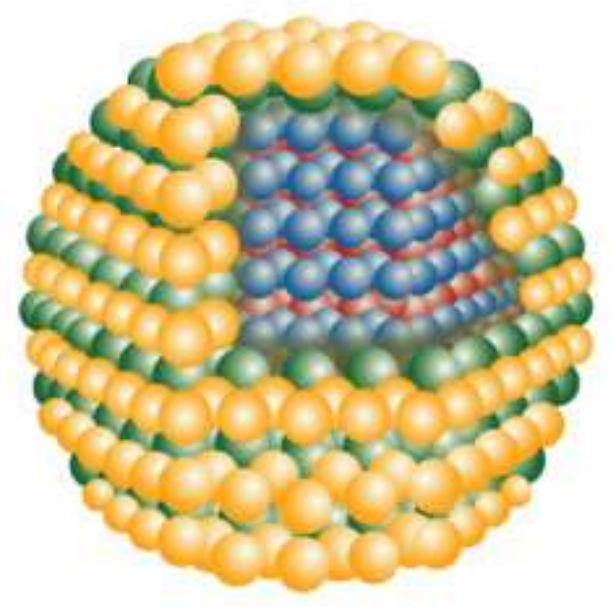

Figure 2-2: CdSe/ZnS core-shell quantum dot (modified from [45]). Red and blue spheres represent the Cadmium and Selenium atoms. Yellow and green spheres represent the Zinc and Sulphur atoms.

(a.)

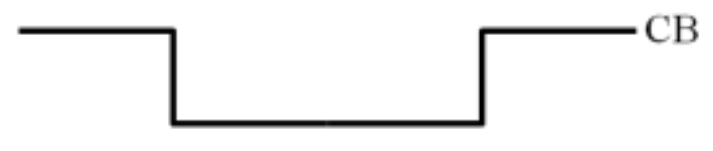

Shell
Shell

Core

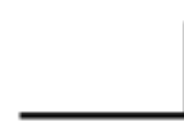

(b.)

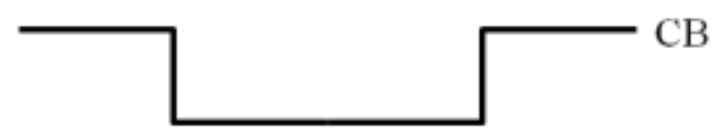

Shell
Shell

Core

VB

Figure 2-3: Cross sectional view of the bottom of the conduction band (CB) and top of the valence band (VB) for (a.) type-I and (b.) type-II QD heterostructures. 


\subsection{Quantum Dot Theory}

From quantum mechanics, electron and hole wave functions are confined by the boundaries set by a potential well. For a particle confined to a well its motion becomes quantized and its energy increases. Similarly, in semiconductor quantum dots, the confinement of excitons is set by the boundaries of a spherical potential well. In this study, two quantum mechanical models are presented: the effective mass approximation (EMA) model and effective mass with Coulomb interaction model. A more comprehensive theoretical discussion into the energy states of carriers in quantum dots is provided in reviews by Klimov and Murray [46, 47]. An explanation of concepts including energy band gap, exciton, exciton Bohr radius, density of states, and quantum confinement follows.

\subsubsection{Quantum Confinement}

Quantum confinement causes the increase in the band gap of a material as the particle size decreases. Typically, the particle size is $100 \mathrm{~nm}$ or less. Localization of charge carriers results in quantization of the energy levels. Quantum confinement can occur in 2-D (confinement in two spatial dimensions), 1-D (confinement in one spatial dimension), and 0-D structures (confinement in three spatial dimensions). In 2-D, charge carriers are restricted in one dimension and result in planar structures called quantum wells. 1-D confinement occurs in nanowires. Confinement of charge carriers in all three spatial dimensions, 0-D confinement is only found in quantum dots. 


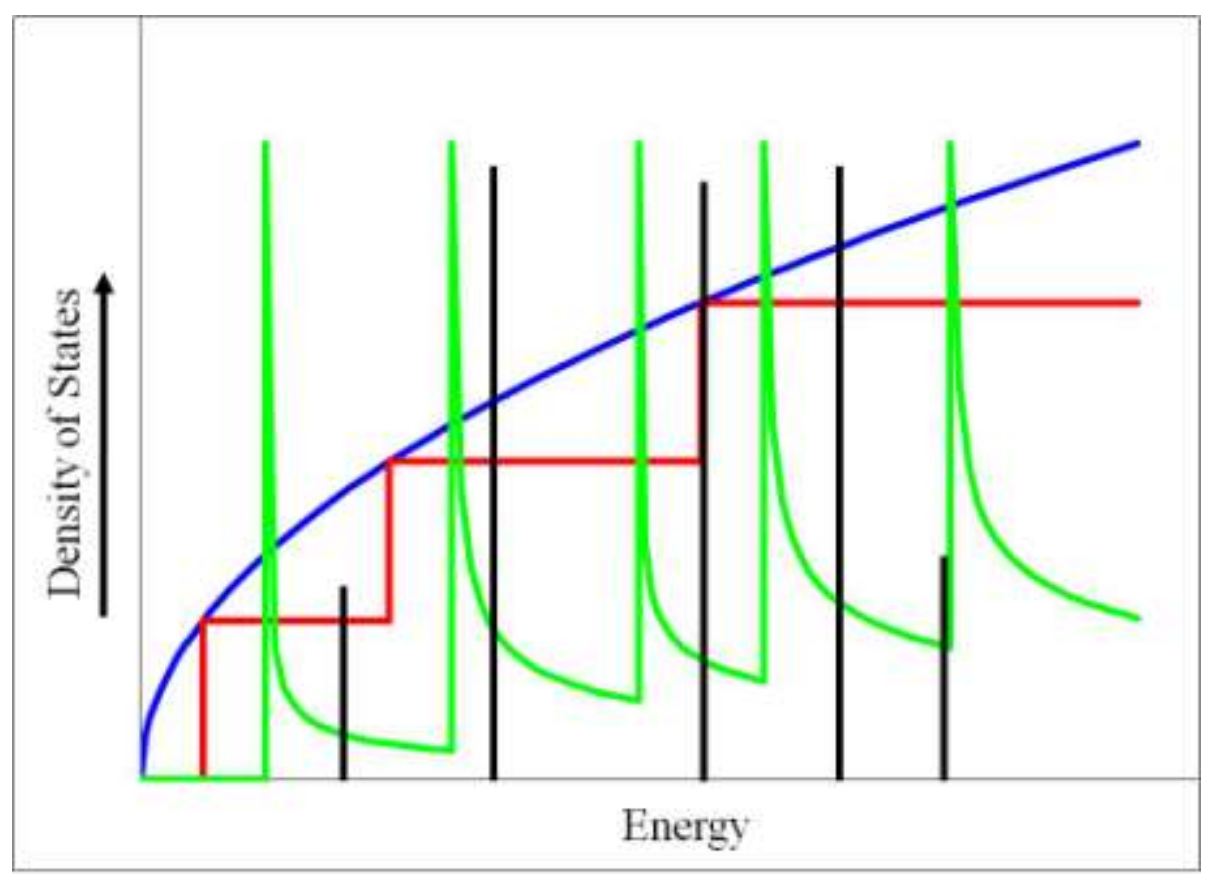

Figure 2-4: Density of states function versus energy plot for 3-D bulk (blue), 2-D quantum well (red), 1-D quantum wire (green), and 0-D quantum dot (black) structures[48].

Similarly to atoms, the energy levels in a QD are quantized due to quantum confinement. Hence, quantum dots are often called "artificial atoms" because the quantized electronic states bear analogy to atomic electronic states. Like atoms, the energy states of quantum dots are discrete. The density of states functions versus energy for 0-D, 1-D, 2-D, and 3D are plotted in Figure 2-4. For a bulk semiconductor (3-D) the density of states goes as $E^{1 / 2}$. Illustrated in Figure 2-4, a series of nearby transitions occurring at slightly different energies in the bulk will be compressed into a single intense transition in a quantum dot. 
The band gap is the forbidden range of electron energy levels located between the top of the valance band and the bottom of the conduction band in a semiconductor or insulator. It is the energy required to create an exciton pair far enough apart such that their Coulomb attraction is negligible. When an electron is promoted from the valance band to the conduction band a hole is created in the valance band. The positive hole forms a bound state with the electron through Coulomb attraction. In quantum dots, this bound state is called a Mott-Wannier exciton. The Mott-Wannier exciton is analogous to the proton and the electron of the hydrogen atom. The spatial occupation of this hydrogen atom-like system can be expressed in terms of the exciton Bohr radius $\left(a_{\mathrm{b}}\right)$ :

$$
a_{b}=\frac{\hbar^{2} \varepsilon}{e^{2}}\left[\frac{1}{m_{e}}+\frac{1}{m_{h}}\right]
$$

where $\hbar=\frac{h}{2 \pi}$ is the reduced Planck's constant, $\varepsilon$ is the dielectric constant, e represents the charge of an electron, and $\mathrm{m}_{\mathrm{e}}\left(\mathrm{m}_{\mathrm{h}}\right)$ are the electron (hole) effective mass. The CdSe effective mass of the electron and hole have been measured to $0.13 \mathrm{~m}_{\mathrm{o}}$ and $0.45 \mathrm{~m}_{\mathrm{o}}$ respectively, where $m_{o}$ is the free electron mass [49]. The effective mass dictates the quantum confinement regimes in quantum dots. The confinement regimes are classified as weak, intermediate, and strong confinement. Weak confinement regime corresponds to the case in which the QD particle size is comparable to the de Broglie wavelength but larger than the Bohr radius of the exciton $\left(a_{\mathrm{b}}<\mathrm{r}_{\mathrm{QD}} \sim \lambda\right.$, where $a_{\mathrm{b}}$ is the exciton Bohr radius, $\mathrm{r}_{\mathrm{QD}}$ is the size of the quantum dot, and $\lambda$ denotes the de Broglie wavelength). Intermediate confinement regime means that $a_{\mathrm{b}} \sim \mathrm{r}_{\mathrm{QD}}$. The strong confinement regime 
describes the case when physical dimensions of the nanocrystal are smaller than the exciton Bohr radius ( $\left.\mathrm{r}_{\mathrm{QD}}<a_{\mathrm{b}}\right)$. The optical properties of quantum dots are most affected and studied in the strong confinement regime. CdSe has a bulk Bohr radius $\sim 9.6 \mathrm{~nm}$. The $\mathrm{CdSe} / \mathrm{ZnS}$ core-shell quantum dots used in this thesis exhibit strong quantum confinement.

In the strong confinement regime, the effective mass approximation (EMA) quantitatively predicts a wavelength blue-shift in the absorption spectrum with respect to bulk. Since the physical dimensions of the quantum dot can be smaller than the exciton diameter, the physics of QDs is describes as an infinite 3-dimensional potential well or "particle in a sphere". Assuming spherical dots, the Wannier exciton is described by the hydrogen-like Hamiltonian as [40, 50]:

$$
H=-\frac{\hbar^{2}}{2 m_{e}^{*}} \nabla_{e}^{2}-\frac{\hbar^{2}}{2 m_{h}^{*}} \nabla_{h}^{2}-\frac{e^{2}}{\varepsilon\left|\vec{r}_{e}-\vec{r}_{h}\right|}+V
$$

The first two terms in Equation 2.2 represent the kinetic energies of the electron and the hole, where $m_{e}^{*}\left(m_{h}^{*}\right)$ is the effective mass of the electron (hole). The third term is the Coulomb potential energy, where $\varepsilon$ is the relative dielectric constant and $\overrightarrow{r_{e}}\left(\overrightarrow{r_{h}}\right)$ is the electrons (hole) coordinate. The last term takes into account the effect of the surrounding matrix. It is described by a spherical potential well of radius $a$ where,

$$
V=\left\{\begin{array}{l}
V_{o} \quad \text { if } r \leq a \\
0 \text { if } r>a
\end{array}\right.
$$


Ignoring the Coulomb interaction, the electron and the hole act as two independent particles inside a spherical well. The wave function is given by [51, 52]:

$$
\psi\left(\vec{r}_{e} \vec{r}_{h}\right)=A \phi\left(\vec{r}_{e}\right) \phi\left(\vec{r}_{h}\right) \exp \left(-\frac{\vec{r}_{e, h}}{\alpha}\right)
$$

where, $\mathrm{A}$ is the normalization constant, $\phi\left(\mathrm{r}_{\mathrm{e}}\right)$ and $\phi\left(\mathrm{r}_{\mathrm{h}}\right)$ are the electron and hole wave functions, and $\alpha$ is a constant. Within the effective mass model, the QD energy band gap is approximated:

$$
E=E_{g(\text { bulk })}+\frac{\hbar^{2} \pi^{2}}{2 R^{2}}\left(\frac{1}{m_{e}^{*}}+\frac{1}{m_{h}^{*}}\right)
$$

where $\mathrm{E}_{\mathrm{g}(\text { bulk })}$ is the bulk material band gap, $\hbar$ is the reduced Planck's constant, $\mathrm{R}$ is the particle size, and the effective mass of the electron (hole) is denoted by $m_{e}^{*}\left(m_{h}^{*}\right)$. From EMA, the exciton energy is quantized and the QD band gap increases as the dot radius decreases. However, the $1 / \mathrm{R}^{2}$ term only approximates the kinetic energy contribution to the e-h pair total energy [22] . Since the electron and hole attract each other, confining them lowers their net potential energy and the Coulomb interaction energy decreases as 1/R. Brus derived the effective mass model with Coulomb interaction $[40,53]$ :

$$
E=E_{g(b u l k)}+\frac{\hbar^{2} \pi^{2}}{2 R^{2}}\left(\frac{1}{m_{e}^{*}}+\frac{1}{m_{h}^{*}}\right)-\frac{1}{R}\left(\frac{1.786 e^{2}}{4 \pi \varepsilon_{o} \varepsilon_{r}}\right)
$$

where, as define in Equation 2.5, $\mathrm{E}_{\mathrm{g}}$ (bulk) is the band gap of bulk CdSe, $\hbar$ is the reduced Planck's constant, $\mathrm{R}$ is the particle size, and the effective mass is denoted by $m_{e}^{*}\left(m_{h}^{*}\right)$. In the third term, e represents the electron charge, $\varepsilon_{0}$ is the permittivity of free space, and 
$\varepsilon_{\mathrm{r}}$ is the relative dielectric constant. The QD energy band gap approximation takes into account the bulk energy band gap. The density of states in a bulk semiconductor is low at the band edge. The density of states in a quasi-continuum of energy levels increases rapidly above the conduction band edge and below the valence band edge. This gives rise to a structureless absorption continuum in a bulk CdSe semiconductor. The situation is different for the CdSe quantum dot. Due to a limited number of atoms $(\sim 100-10,000$ atoms) in a quantum dot, discrete atomic-like energy levels show in the conduction and valence band edges. Figure 2-5 gives a physical picture of the bulk semiconductor and QD band structures.

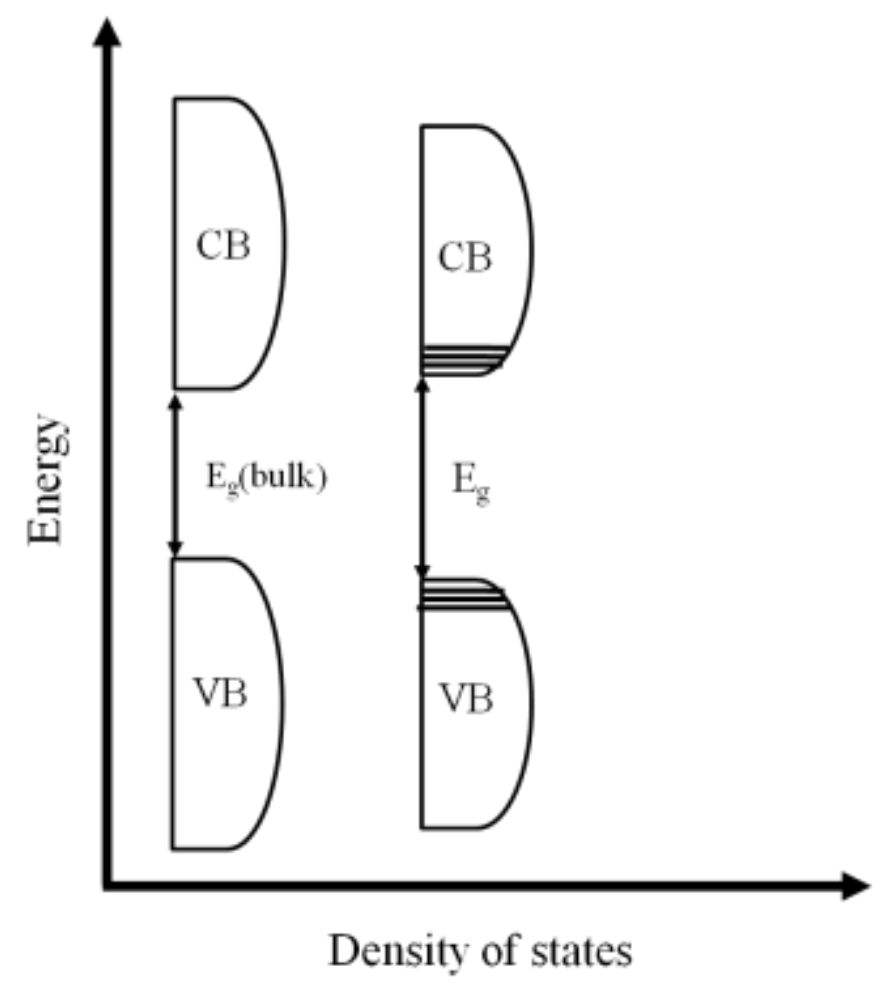

Figure 2-5: Schematic of the band structure of a bulk semiconductor (left) and a quantum dot (right). 
Over the past two decades, extensive experimental and theoretical research has been carried out to refine the EMA as well as propose new quantum mechanical models that better match experimentally determined band gap energies to CdSe QD particle sizes. Murray et al. argue that EMA overestimates the first absorbance peak energy for CdSe quantum dots with radii smaller than $4 \mathrm{~nm}$ [54]. Krishana et al. have proposed pseudopotential models for heavy-hole light-hole effect and band simulation for $\mathrm{CdSe}$ [55]. In more recent work, Zunger proposed that an accurate description of the size dependence of the optical band gap should include polarization effects [56]. Those models are not presented here because the EMA well quantifies the CdSe/ZnS quantum dot sizes when compared against experimental results presented in Chapter 3. The surface effect and the thermal effect for QD-based luminescence thermometry are considered below.

\subsubsection{Surface Effect}

The large surface to volume ratio in semiconductor quantum dots results in strong influence of surface states on the optical properties of QDs. Small particle sizes can result in surface imperfections and aggregates that act as traps for photogenerated electrons and holes. These factors contributed to low yields $(\sim 5-15 \%)$ of photoluminescence in CdSe quantum dots. Passivation is a chemical process in which the core surface atoms are bonded to another material. The shell provides efficient passivation of the surface trap states which gives rise to an enhanced fluorescence quantum yield upwards of $50-80 \%$ [57-59] . In addition, the shell provides a physical barrier between the optically active core and the surrounding medium which makes QDs 
less sensitive to environmental changes such as photo-oxidation or the presence of gases $[57,60]$. Quantum dots capped with an inorganic shell are more robust and have greater tolerance to processing necessary for device applications. A careful characterization of overgrowth of CdSe nanocrystals with $\mathrm{ZnS}$ was carried out by Dabbousi [61]. Figure 2-6 shows the absorption spectra for different size bare CdSe dots in hexane solution compared against the absorption measurements taken on $\mathrm{CdSe} / \mathrm{ZnS}$ core-shell QDs. 


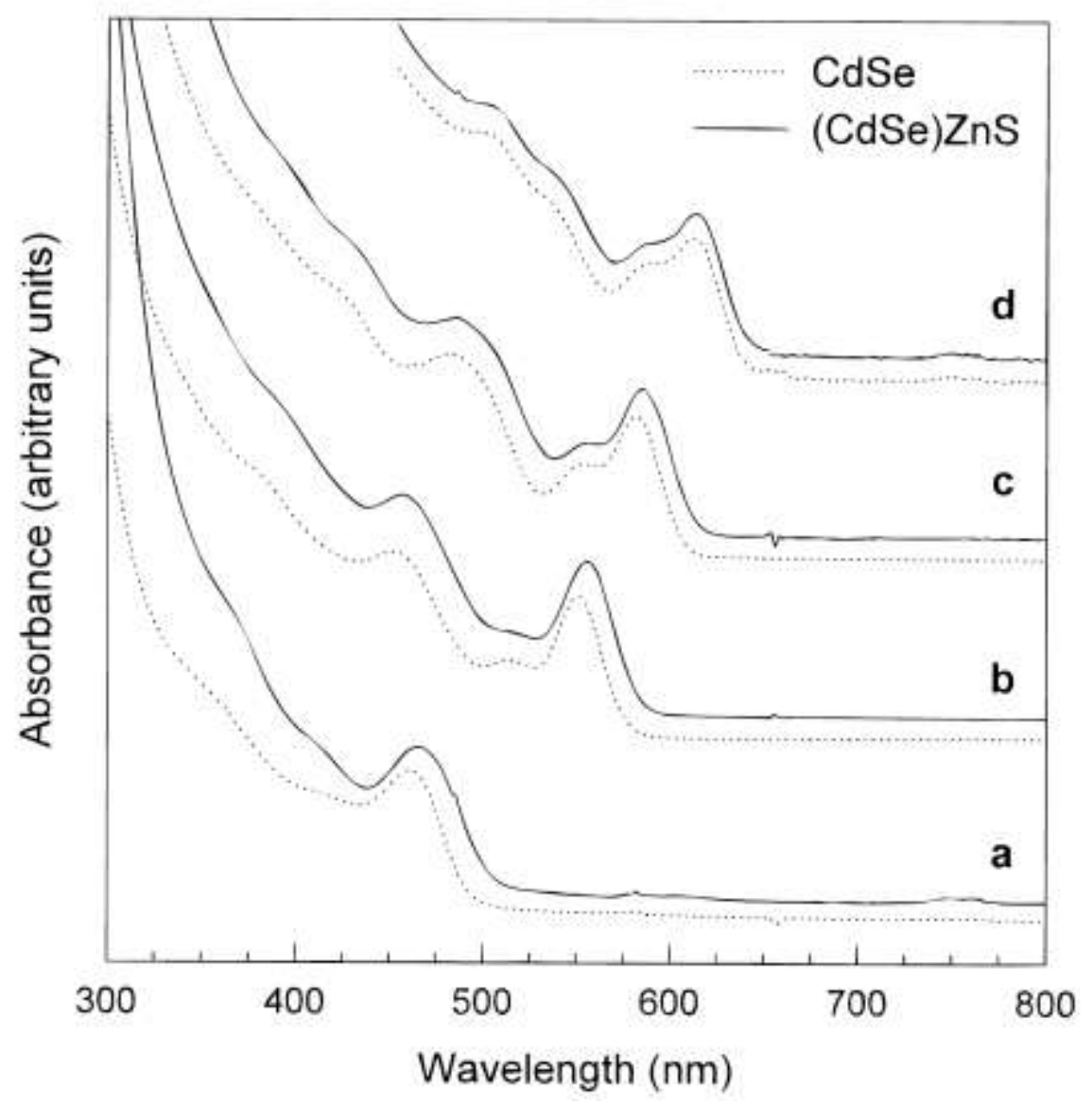

Figure 2-6: Absorption versus wavelength for bare $\mathrm{CdSe}$ (dashed lines) and $\mathrm{CdSe}(\mathrm{ZnS})$ (solid lines) a $2.3 \mathrm{~nm}, \mathbf{b} 4.2 \mathrm{~nm}, \mathbf{c} 4.8 \mathrm{~nm}$, and d $5.5 \mathrm{~nm}$ quantum dots. Measurements were taken at room temperature [61]. 


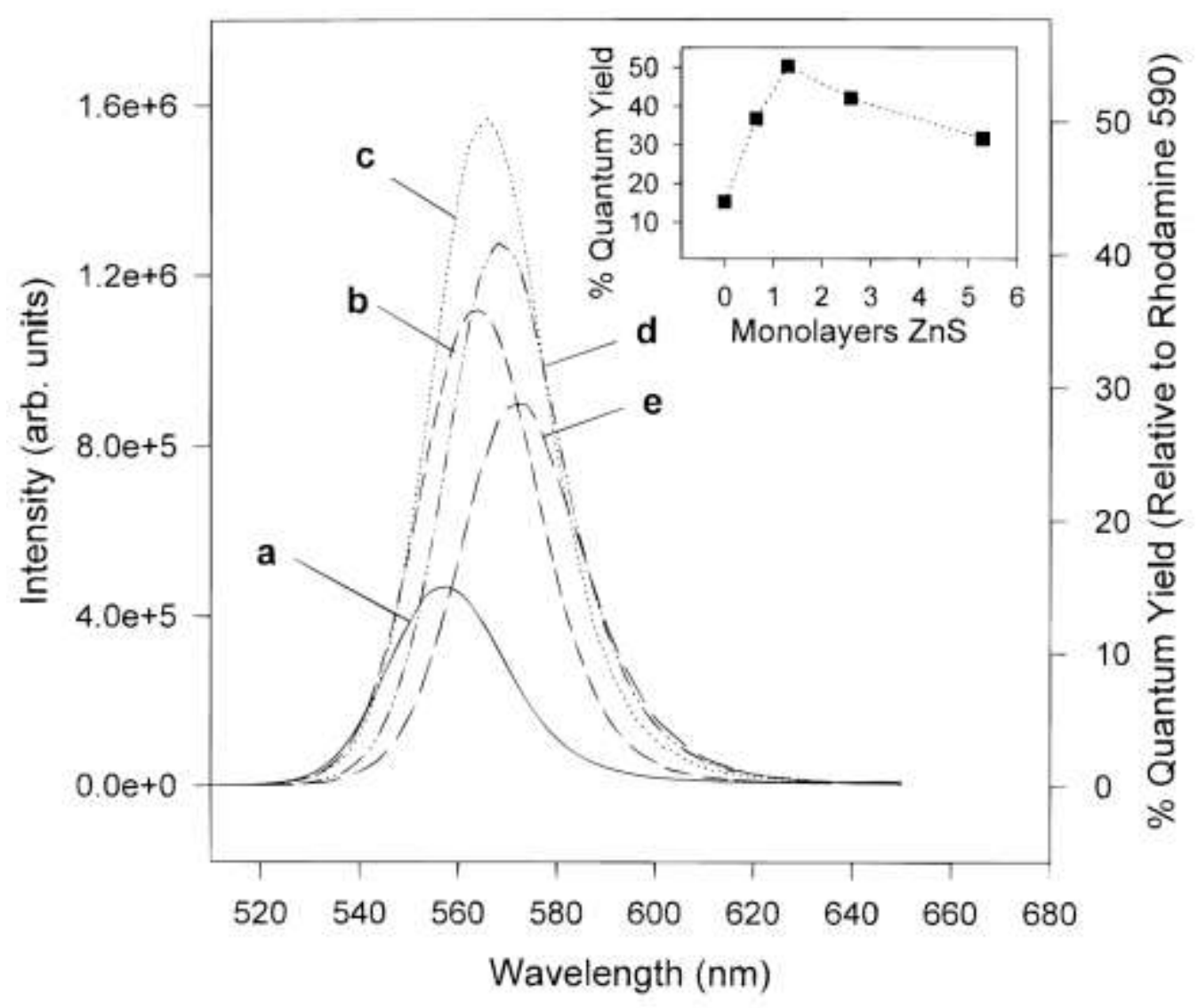

Figure 2-7: Photoluminescence versus wavelength for $4.2 \mathrm{~nm} \mathrm{CdSe} / \mathrm{ZnS}$ dots with a 0 , b $0.65, \mathbf{c} 1.3, \mathbf{d} 2.6$, and $\mathbf{e} 5.3$ monolayer $\mathrm{ZnS}$ coverage. Inset is the Q.Y. versus monolayers of $\mathrm{ZnS}$ shell material. Measurements were taken at room temperature [61]. 
A 1 monolayer $(3.1 \AA$ ) thick shell is optimum. Enhanced absorption and a blue-shift in the absorbance are reported for the $\mathrm{ZnS}$-capped CdSe dots. In Figure 2-7 the PL quantum yield is charted as a function of shell thickness. A 50\% enhancement in quantum yield is reported with a 1.3 monolayer shell. PL quenches with thicker shells.

The dielectric effect is another interesting surface property that manifests in quantum dots. The interface between the QD and the surrounding medium gives rise to interesting confinement effects. When surrounded by dielectrics such air, polymers, or glasses which have much lower refractive indices than the quantum dot, the field intensity near or at the surface can be significantly enhanced compared to the incident intensity $[62,63]$. This enhancement termed the dielectric confinement effect impacts optical spectroscopy of $\mathrm{CdSe} / \mathrm{ZnS}$ quantum dots. Torchynska et al. drop cast CdSe/ZnS QDs onto silicon wafers and observed no change with temperatures from 10-300 K in the 2.37 and $2.68 \mathrm{eV}$ PL bands. Torchynska explained the constant PL emission as a result of electron-hole recombination via interface states [63].

\subsubsection{Thermal Effect}

The melting temperature of low-dimensional structures depends on size. For freestanding particles, thin films, and nanowires the melting temperature decreases with decreasing particle size [64]. In contrast, the melting temperature of embedded nanoparticles can be higher than the bulk melting point for certain matrixes [65]. The enhancement or depression of the melting temperature of embedded nanoparticles depends on the epitaxy between the nanoparticle and the embedding matrix. Nanda et al. reported that superheating occurs when nanomaterials with lower surface energy are 
embedded into a matrix material of higher surface energy. The relationship between the melting temperature of nanoparticles and their corresponding size is given by [66]:

$$
\frac{T_{m}(r)}{T_{m}(\infty)}=\exp \left[-\frac{(\alpha-1)}{\left(r / r_{o}-1\right)}\right]
$$

where $T_{m}(r)$ and $T_{m}(\infty)$ are the melting temperatures of the QDs with radius $r$ and bulk material. The critical radius at which all atoms are located on the surface of the particle is $r_{o}$ and $\alpha$ is the ratio of the mean square displacement of atoms on the surface. If $\alpha>1$, $\mathrm{T}_{\mathrm{m}}(\mathrm{r}) / \mathrm{T}_{\mathrm{m}}(\infty)<1$, then $\mathrm{T}_{\mathrm{m}}(\mathrm{r})$ decreases as $\mathrm{r}$ decreases. When $\alpha<1, \mathrm{~T}_{\mathrm{m}}(\mathrm{r}) / \mathrm{T}_{\mathrm{m}}(\infty)>1$, then $T_{m}(r)$ increases as $r$ increases. In this research, the thermal effect is investigated for two material systems. The temperature dependent optical properties of $\mathrm{CdSe} / \mathrm{ZnS}$ quantum dots are investigated in a low temperature dielectric polymethlmethacrylate (PMMA). The temperature dependent optical properties of CdSe/ZnS quantum dots embedded in a high temperature resistant silicon dioxide $\left(\mathrm{SiO}_{2}\right)$ dielectric is characterized via quantum dot luminescence thermometry. 


\subsection{Quantum Dot Luminescence Thermometry}

\subsubsection{Absorption Spectroscopy}

As a beam of light passes through a bulk semiconductor material it attenuates due to the interaction of light with the material in two basic ways: scattering and absorption. Figure 2-8 illustrates a configurational coordinate diagram of absorption processes in direct bandgap semiconductor quantum dot CdSe. For light incident normal to a quantum dot thin film of thickness $x$, the fraction of light absorbed by the quantum dots is given by the Beer-Lambert Law:

$$
I=I_{o} \exp (-\alpha x)
$$

where I is the intensity of the transmitted beam, $I_{0}$ is the incident beam, and $\alpha$ represents the linear absorption coefficient. For unpolarized light at normal incidence the reflected light is approximated by $R=(n-1)^{2} /(n+1)^{2}$, where $\mathrm{n}$ is the index of refraction of the material. The transmitted light reduces to:

$$
I=I_{o}(1-R) \exp (-\alpha x)
$$

For a nonisotropic material the absorption coefficient can vary with direction. Quantum dots are taken as isotropic. The absorption coefficient is related to the absorption cross section, $\sigma_{\mathrm{a}}$ by:

$$
\alpha=\sigma_{a} N
$$




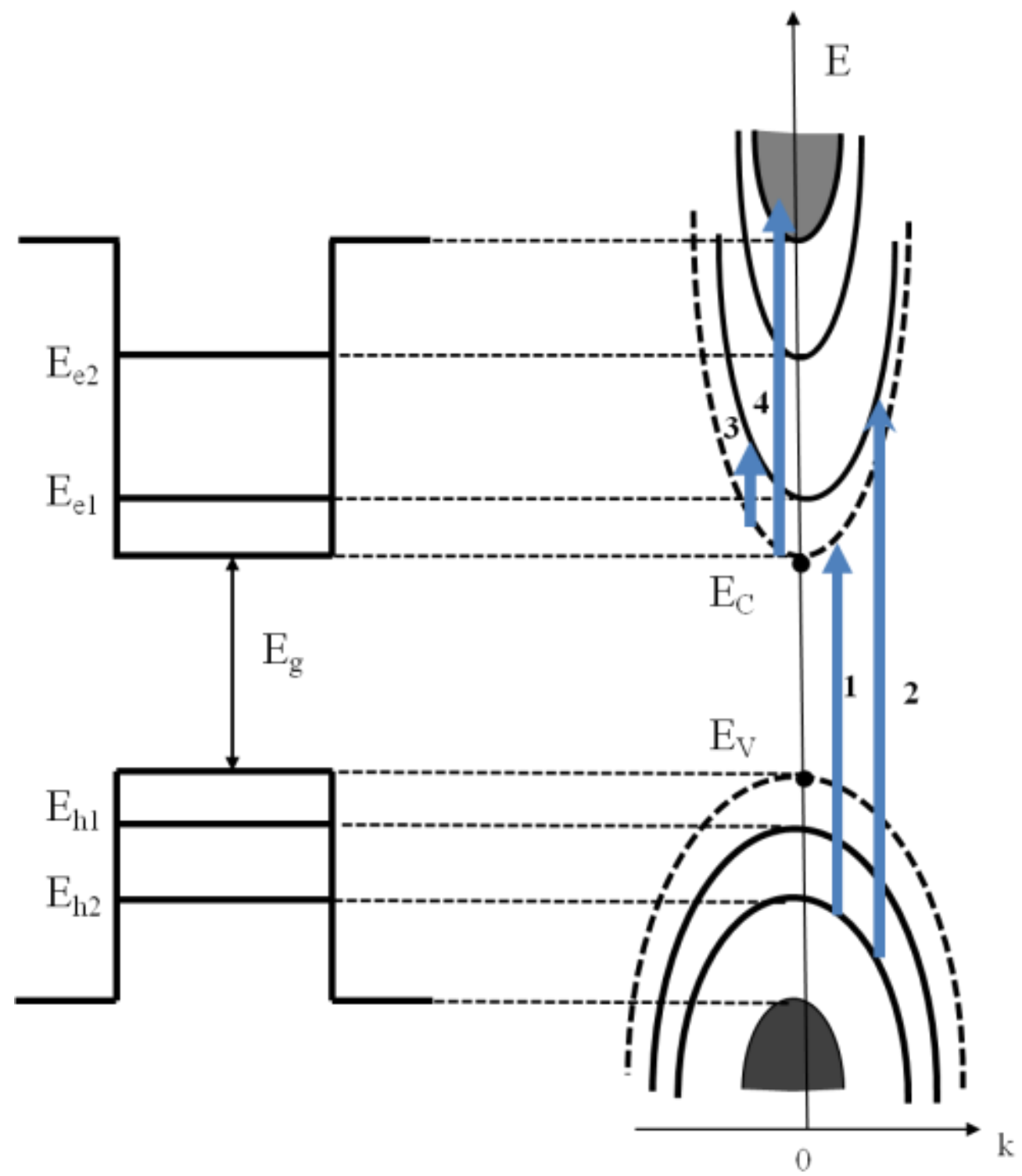

Figure 2-8: Configurational coordinate diagram for CdSe quantum dots. The blue arrows indicate (1), (2) direct interband transitions, (3) direct intra-subband transitions, and (4) transitions from quantum-confined states to continuum states. 
where, $\mathrm{N}$ is the concentration of absorbers. For molecular-like systems in solution such as $\mathrm{CdSe} / \mathrm{ZnS}$ quantum dots, the form of Beer's Law commonly used is:

$$
A=\varepsilon C l
$$

where $\mathrm{A}$ is the optical density, $\varepsilon$ is the molar extinction coefficient, $\mathrm{C}$ is the concentration and $l$ is the optical path length. Equation 2.9 can be written in terms of A, where $\mathrm{A}=\log \left(\mathrm{I}_{\mathrm{o}} / \mathrm{I}\right)$. Equating 2.8 to 2.11 and solving for the absorption cross section from Eqn. 2.10 gives:

$$
\sigma_{a}=\frac{2.3026(1000)}{N} \varepsilon
$$

where $\mathrm{C}(\mathrm{mol} / \mathrm{liter})$ and $\mathrm{N}\left(\mathrm{mol} / \mathrm{m}^{3}\right)$. For small particles, the absorption cross section is directly proportional to the nanocrystal volume, V. For an isotropically polarized, spherical particle QD ensemble the cross section is expressed in local field approximation as $[67,68]$ :

$$
\sigma_{a}=V \frac{n_{1}}{n_{2}}|f(\omega)|^{2} \alpha_{b u l k}(\omega)
$$

where, $V=\frac{4}{3} \pi r^{3}$ is the volume of the nanoparticle with radius $\mathrm{r}, \mathrm{n}_{1}, \mathrm{n}_{2}$ are the real parts of the nanoparticle and solvent refractive index, respectively. The absorption coefficient of the bulk material is denoted by $\alpha_{\text {bulk }}$ and $f$ denotes the local field factor. The local field 
correction factor accounts for the difference in the electric field inside and outside of the nanoparticle and is defined as:

$$
f=\frac{3 \varepsilon_{1}}{2 \varepsilon_{1}+\varepsilon_{2}}
$$

where, $\varepsilon_{1}$ and $\varepsilon_{2}$ are the surrounding medium and quantum dot relative permittivity.

\subsubsection{Photoluminescence Spectroscopy}

Fluorescence is the emission of light from the $S_{1}$ singlet-excited state in which the electron in the excited orbital is paired (opposite sign) to another electron in the groundstate orbital. The spontaneous decay from the excited state to a lower level via emission of a photon on a timescale less than $10^{-9} \mathrm{sec}$ is called fluorescence. Phosphorescence occurs from the lowest triplet state on a timescale $\sim 10^{-3}-10 \mathrm{~s}$. According to Kasha's Rule, the electron first returns to the lowest vibrational level of the first excited state via non-radiative decay. The electron can then return to the ground state by various deexcitation pathways. The Jablonski energy level diagram in Figure 2-9 shows the pathways. 


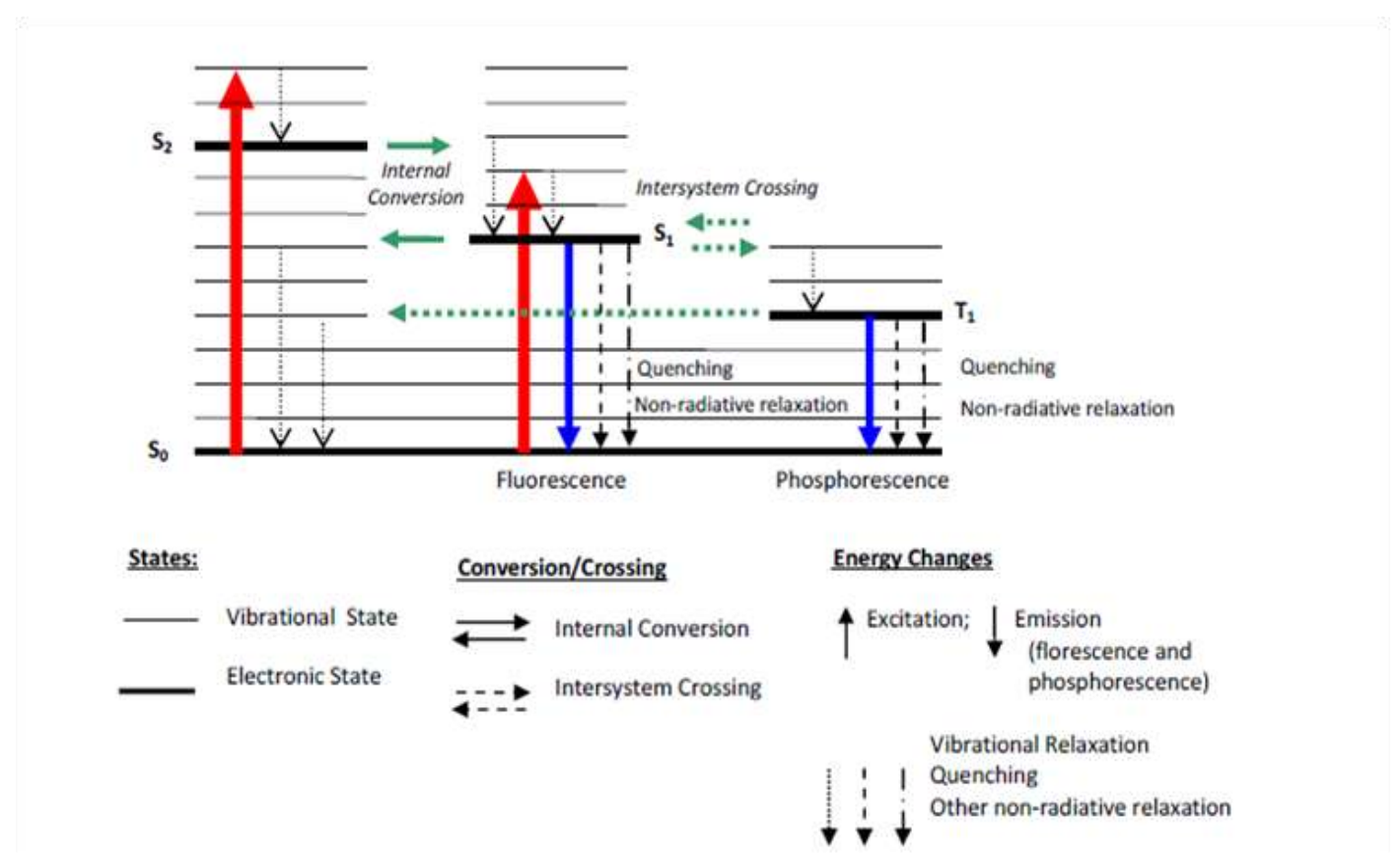

Figure 2-9: Jablonski energy level diagram showing the photoluminescence processes [8].

In the excited state, the electron occupies an electronic energy level according to the temperature dependent Boltzmann probability function. Under thermal equilibrium, the population of energy levels is given:

$$
\frac{N_{1}}{N_{0}}=\exp \left[-\frac{\left(E_{1}-E_{0}\right)}{k_{B} T}\right]
$$

where $\mathrm{N}_{1}$ and $\mathrm{N}_{0}$ represents the number of atoms in the excited and ground states, $\mathrm{E}_{1}$ and $\mathrm{E}_{0}$ are the energies of the excited state and ground state, $\mathrm{k}_{\mathrm{B}}$ is Boltzmann's constant, and $\mathrm{T}$ is the temperature. Some energy is lost through heat and lattice vibrations such that the 
emitted energy is less than the excitation energy. The energy difference $\Delta=h v_{a b s}-h v_{e m}$ is a measure of the Stokes shift. From the Stokes shift an understanding of quantum efficiency is obtained.

An intensity $I_{o}$ enters the quantum dot and an intensity I passes through. The emitted intensity $\mathrm{I}_{\mathrm{em}}$ is written:

$$
I_{e m}=\eta\left(I_{o}-I\right)
$$

where $\mathrm{I}_{e m}, \mathrm{I}_{0}, \mathrm{I}$ are in photons per second and $\eta$ is the quantum efficiency (Q.E.). Q.E. represents the ratio between the emitted photons and absorbed photons. The Q.E. exhibits a temperature dependence. Spectral transition energy, line intensity, full-width half maximum (FWHM), lifetime, quantum yield, and absorption cross sections can vary with temperature.

The temperature dependence of the energy band gap for bulk semiconductors is described by the Varshni relation [69], which has also been shown to be valid for semiconductor nanostructures $[70,71]$ :

$$
E_{g}(T)=E_{g}(0)-\frac{\alpha T^{2}}{T+\beta}
$$

where $\mathrm{E}_{\mathrm{g}}(0)$ is the transition energy at $0 \mathrm{~K}, \alpha$ and $\beta$ are the material dependent parameters termed Varshni's coefficients. Specifically, $\alpha$ is the temperature coefficient and $\beta$ is a fitting parameter close to the Debye temperature of the crystal. 
The band broadening with increasing temperature in semiconductor quantum dots is explained by scattering with acoustic and optical phonons. The temperature dependence of the spectral linewidth due to exciton scattering with acoustic and longitudinal optical (LO) phonons is described as the sum of three terms [72-74]:

$$
\Gamma(T)=\Gamma_{i n h}+\sigma T+\Gamma_{L O}\left(\mathrm{e}^{E_{L O} / k_{B} T}-1\right)^{-1}
$$

where $\Gamma_{i n h}$ is the inhomogeneous line width that is temperature independent. Inhomogeneous line width results from fluctuation in size, shape, and compositions of the quantum dot materials. The exciton acoustic phonon coupling coefficient is denoted by $\sigma, \Gamma_{\mathrm{LO}}$ represents the exciton-LO coupling coefficient, $\mathrm{E}_{\mathrm{LO}}$ is the LO-phonon energy, $\mathrm{k}_{\mathrm{B}}$ is Boltzmann's constant, and $\mathrm{T}$ is temperature.

\subsubsection{Time-resolved Photoluminescence Spectroscopy}

Pulsed wave excitation promotes a density of quantum dot centers denoted by $\mathrm{N}$ into the excited state. The excited QD centers can decay to the ground state by nonradiative and radiative processes, giving rise to a decay-time intensity signal. Timeresolved photoluminescence is insensitive to non-uniform excitation and applicable to high temperature environments. The rate of decay population is expressed as:

$$
\frac{d N(t)}{d t}=-A_{T} N(t)
$$




$$
A_{T}=A+A_{n r}
$$

where $A_{T}$ represent the total decay probability, $A$ is the radiative rate, and $A_{n r}$ is the nonradiative rate. The solution to Eqn. 2.19 gives the density of excited quantum dot centers at a time $\mathrm{t}$ :

$$
N(t)=N_{o} \exp \left(-A_{T} t\right)
$$

where, $\mathrm{N}(\mathrm{t})$ is the number of excited QD centers at some time $t$, and $\mathrm{N}_{\mathrm{o}}$ is the initial number of excited centers at $\mathrm{t}=0$. The mean lifetime, $\tau_{m}$ of QD centers in the excited state is given by

$$
\tau_{m}=\frac{1}{A_{T}}
$$

From Eqn. 2.21, the quantum efficiency can now be written

$$
\eta=\frac{A}{A+A_{n r}}
$$

where, for quantum dot center, theory predicts $A_{n r}$ very small and $\eta$ can approach unity. 


\title{
Chapter 3 \\ QUANTUM DOT THIN FILM FABRICATION AND CHARACTERIZATION
}

\begin{abstract}
"The chemist does a mysterious thing when he wants to make a molecule. He sees that it has got that ring, so he mixes this and that, and he shakes it...

And, at the end of a difficult process, he usually does succeed in synthesizing what he wants."
\end{abstract}

Richard P. Feynman

This chapter presents quantum dot film fabrication techniques, experimental procedures, and results. Sol-gel process is used to obtain thin films of CdSe/ZnS quantum dots embedded in $\mathrm{SiO}_{2}$. Two film fabrication procedures were used. $\mathrm{CdSe} / \mathrm{ZnS}: \mathrm{SiO}_{2}$ QD films were fabricated using spin-coating and drop coating techniques. The samples were characterized by atomic force microscopy (AFM). A discussion on the lessons learned through experimentation with colloidal quantum dots is integrated into this chapter.

\subsection{Sol-gel Preparation of CdSe/ZnS Quantum Dot Thin Films}

CdSe/ZnS:PMMA dots were investigated as received. The films were provided by Ocean Nanotechnology, LLC. A wet chemical synthesis method that included blending of $\mathrm{CdSe} / \mathrm{ZnS}$ quantum dots with the polymer was used. The technical details were proprietary per Ocean Nanotechnology. The concentration of QDs in the PMMA was $5 \mathrm{mg} / \mathrm{mL}$. 
High optical quality quartz wafers (14 mm diameter x $3 \mathrm{~mm}$ thickness) were used as substrates for the $\mathrm{CdSe} / \mathrm{ZnS}: \mathrm{SiO}_{2}$ films The wafers were manufactured by Advantiv Corporation. Each wafer was cleaned using a four-step cleaning process. First, the wafer was bathed in acetone. An ethanol and isopropyl alcohol (IPO) rinse followed. Care was taken in the drying process to ensure no residue was left on the wafer. Holding the wafer with plastic tongs at about a $45^{\circ}$ angle, the wafer was spray-dried with Nitrogen gas. The residue slid downward, off the wafer. The wafer dried on air for several minutes prior to film deposition. A $50 \mathrm{mg}$ vial of powdered colloidal CdSe/ZnS quantum dots were purchased from Ocean Nanotechnology. Spin-on-glass (SOG) was purchased from Filmtronics Incorporated. Figure 3-1 outlines the procedures used to prepare $\mathrm{CdSe} / \mathrm{ZnS}: \mathrm{SiO}_{2}$ films using the spin-coating technique. CdSe/ZnS samples were measured out using a Mettler-UMT2 microbalance. The QD sample was then added to 35-50 $\mu \mathrm{L}$ of the SOG solution. The colloidal suspension was mounted into an ultrasonic bath for 90 minutes. A disposable-microliter pipette was used to dispense $25-50 \mu \mathrm{L}$ solution onto the quartz substrate. The sol-gel was spin-coated onto the substrate. Film thickness versus spin coater speed is plotted in Figure 3-2. The QD-silica coating was subjected to thermal annealing. The baking times and temperatures ranged from 140$250^{\circ} \mathrm{C}$ depending on the film thickness. After heat treatment, the film cooled to a thin glass silica film. Clusters of quantum dots were visible with the unaided eye. The glass functioned to protect the QDs from oxidation (reference Chapter 6). 
PROCESSING

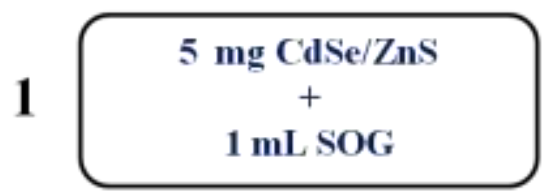

2 Sonicate for 1.5 hours.

3 Mount wafer onto chuck. Stability Run. $4 \begin{aligned} & \text { Deposit } 25 \mu \mathrm{L} \text { of sol-gel. } \\ & \text { Spin Coat. }\end{aligned}$

$5 \begin{aligned} & \text { Transfer wafer to hot } \\ & \text { plate. } 20 \text { minute bake at } \\ & 240^{\circ} \mathrm{C}\end{aligned}$
OBSERVATIONS

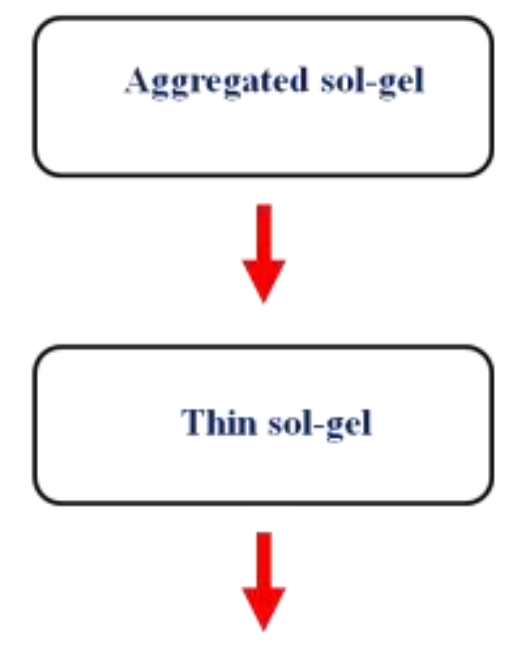

Nanocomposite $\mathrm{SiO}_{2}$ doped thin film with quantum dots. Ready for characterization.

Figure 3-1: Preparation of $\mathrm{CdSe} / \mathrm{ZnS}: \mathrm{SiO}_{2}$ thin films by spin coating process. 


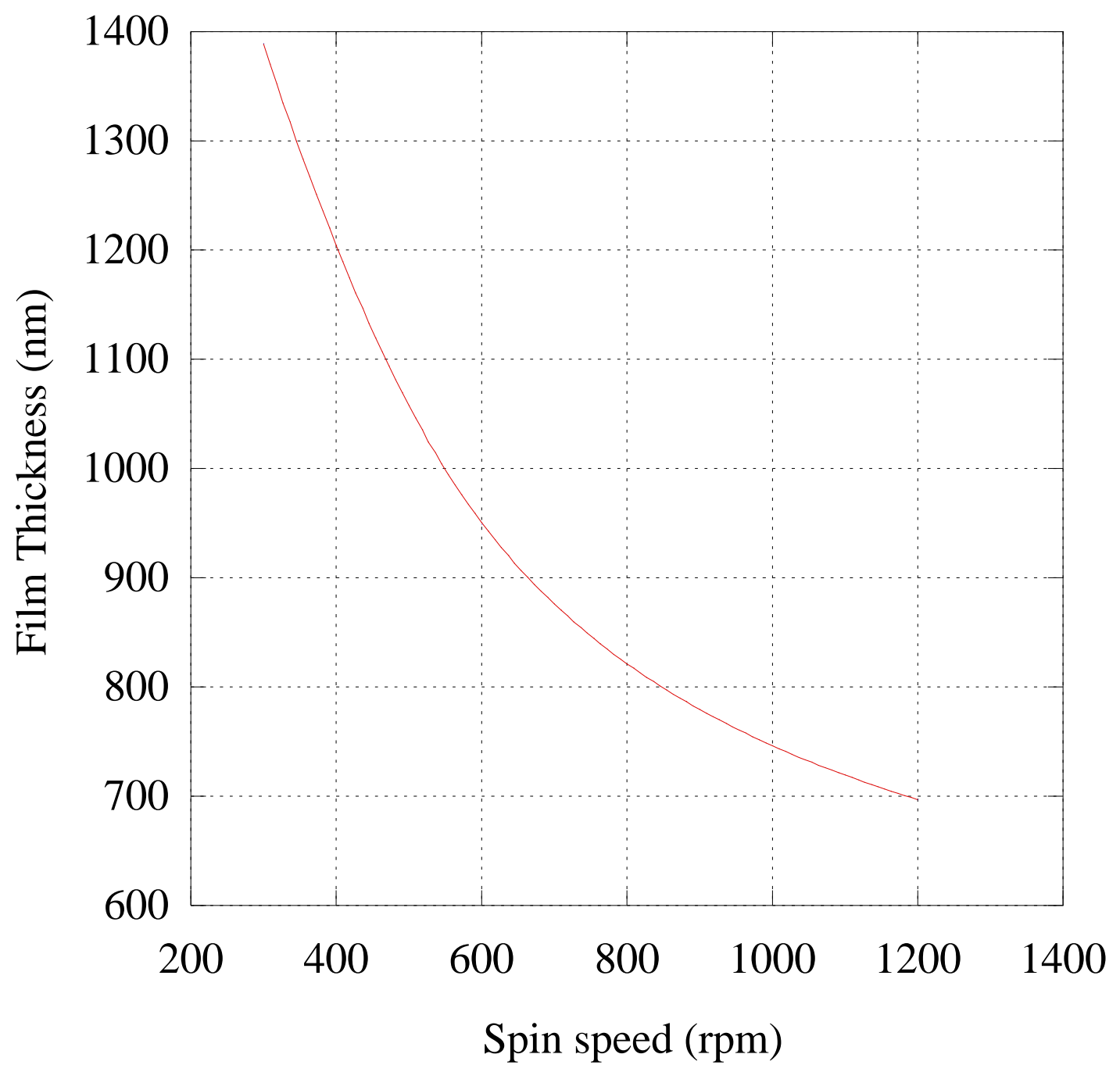

Figure 3-2: Calibration curve generated for spin-coating $\mathrm{CdSe} / \mathrm{ZnS}: \mathrm{SiO}_{2} \mathrm{QD}$ films. 
A significant amount of quantum dots are wasted using spin coating. This is a key limitation given the expense of commercial quantum dots. A less wasteful film fabrication method was accomplished using drop coating. Drop coating also gave potential for fabricating thicker films than spin coating.

Drop casting was initially carried out with $\mathrm{CdSe} / \mathrm{ZnS}$ quantum dots dispersed in toluene. The concentration of quantum dots in toluene is $2.5 \mathrm{mg} / \mathrm{mL}$. When dispensed from the pipette the sol drops onto the substrate to a round liquid droplet. As the droplet begins to dry, a slight curvature is observed. After 1 hour the droplet dries to a cluster distribution of the CdSe/ZnS quantum dots with the bulk of the QDs densely packed at the outer edge of the droplet. Low-powered microscope observations of the final distribution after evaporation of toluene reveals multiple layers of CdSe/ZnS QDs at the periphery of the area originally occupied by the droplet. As the $\mathrm{CdSe} / \mathrm{ZnS}$ quantum dot droplet dries, the droplet contact line remains pinned and the fluid carrying the QDs flows outward from the center of the drop. The QDs are transported in the bulk suspension and along the air-liquid interface settling into a multi-ring pattern after solvent evaporation. This observation is explained as the coffee ring effect.

The physics of the coffee-ring effect has focused on micrometer-scale and larger particles [75-77]. Few literature references have surfaced on the coffee-ring phenomenon in colloidal CdSe/ZnS quantum dots [78]. Since large batches of quantum dots can be synthesized via colloidal chemistry, colloidal semiconductor QDs have catalyzed the broad use of nanometer-sized particles. Due to this scalability and the convenience of bench-top laboratory set-ups, colloidal synthetic methods are readily used. However, preparation of large scale, inexpensive ordered CdSe/ZnS QD particle 
arrays on a substrate remains a significant obstacle to practical quantum dot-based device applications. In a Nature 2011 paper, Yunker et al. attacked the coffee-ring problem by changing the size of polystyrene (PS) particles from spheres to ellipsoids [79]. Experimental observations show that micrometer-sized PS spheres stretched asymmetrically and dispersed in water resist capillary flow. An increased inter-particle attraction deforms the air-water interface such that the PS ellipsoids fell to the bottom of the meniscus and resulted in a uniformly deposited quantum dot film.

As explained in Chapter 2, the quantum dot particle size dictates optical properties. Hence, a traditional approach to reverse or ameliorate the coffee-ring effect was investigated. Capillary flow control was investigated for the CdSe/ZnS quantum dot suspensions. Figure 3-3 (a.) and (b.) are schematics of the liquid meniscus and QD ring formation. A sample deposition cell was configured for drop coating CdSe/ZnS QDs. It consists of two brackets and a micro-chamber. The substrate was mounted between two 7.6 x $2.5 \mathrm{~cm}$ brackets. An o-ring with a $1.07 \mathrm{~cm}$ inner diameter and $1.25 \mathrm{~cm}$ metal gasket were sandwiched between the brackets to create a micro-chamber. The micro-chamber functioned as a reservoir to restrict capillary flow of the sol-gel droplet. Fume hood air flow and laboratory room draft were eliminated by drying the films in an enclosed petri dish. The sol-gel droplet dried to a $\mathrm{CdSe} / \mathrm{ZnS}$ nanocomposite thin film with uniform dispersion at center of the droplet. 


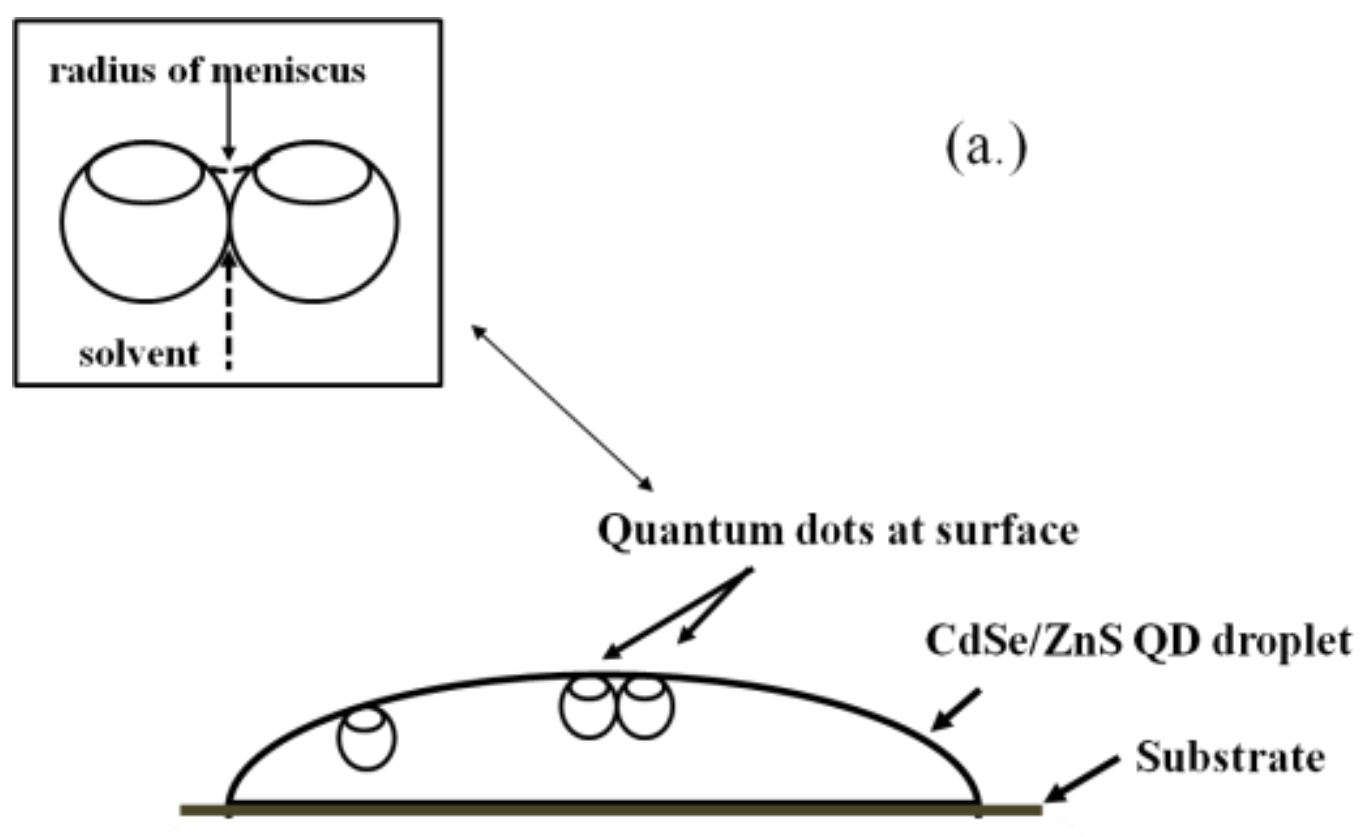

(b.)

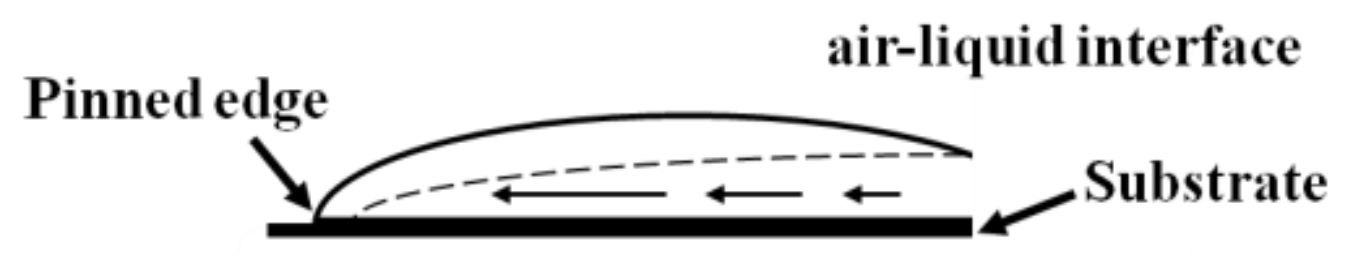

Figure 3-3: (a.) Partially submerged QDs at air-liquid interface and liquid meniscus. The curvature of each local meniscus creates a pressure such that as the droplet dries, the meniscus curvature decreases setting up a pressure gradient which acts as a suction force. (b.) Outer edge QD rings form due to capillary flow induced by pinned edges. 
Sonicating the CdSe/ZnS QD sol for long periods of time ( $>4 \mathrm{hr}$ ) failed to produce an aggregation-free CdSe/ZnS:SOG nanocomposite. Methanol is miscible with SOG. Methanol soluble CdSe/ZnS quantum dots were custom fabricated by Intelligent Optical Systems Company. The concentration of QDs in methanol was $2.5 \mathrm{mg} / \mathrm{mL}$. The PL emission wavelengths were customer-specified to 520, 560, 610, 650, and $680 \mathrm{~nm}$. Figure 3-5 outlines the procedures used to prepare $\mathrm{CdSe} / \mathrm{ZnS}: \mathrm{SiO}_{2}$ films using the dropcasting technique. Film morphology and particle size were characterized using atomic force microscopy (AFM). 
PROCESSING

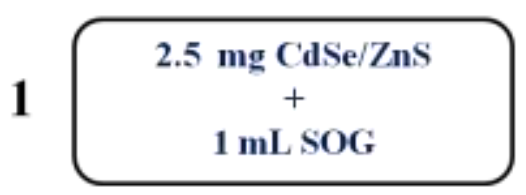

2 Drop cast $2 \mathrm{~mL}$ of sol-gel.

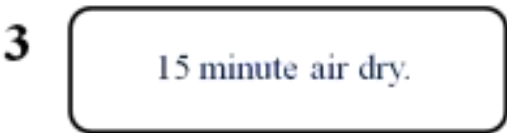

$4 \begin{aligned} & \text { Transfer wafer to hot plate. } \\ & 20 \text { minute bake at } 240^{\circ} \mathrm{C} \text {. }\end{aligned}$
OBSERVATIONS

Thin sol-gel (minimized aggregation
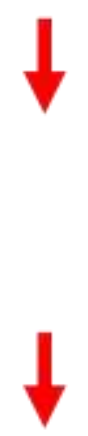

Nanocomposite $\mathrm{SiO}_{2}$ doped thin film with quantum dots. Ready for characterization.

Figure 3-5: Preparation of $\mathrm{CdSe} / \mathrm{ZnS}: \mathrm{SiO}_{2}$ films by drop-coating process. 


\subsection{Characterization using Atomic Force Microscopy (AFM)}

AFM was used to gain both qualitative and quantitative analysis of the CdSe/ZnS quantum dots and $\mathrm{CdSe} / \mathrm{ZnS}: \mathrm{SiO}_{2}$ nanocomposite films. Individual quantum dots and clusters of quantum dots were imaged. Particle shape, size, film thickness, and morphology were characterized. Particle distribution and particle count statistics were recorded.

A Veeco Nanoscope Atomic Force Microscope was used for all measurements. The two main components of the AFM are the probe and the scanner. The probe is the point of interface between the AFM and the sample. The scanner controls the precise position of the probe in relation to the surface, both vertically and laterally. A piezoelectric ceramic changes its geometry under an applied voltage. The voltage is proportional to the mechanical movement. The probe is a micro-fabricated cantilever with a sharp tip. By convention, large $\sim 10 \mathrm{~nm}$ radius Silicon Nitride tips are used. The $\mathrm{CdSe} / \mathrm{ZnS}$ quantum dot particle size was manufactured-specified to 3 - $5 \mathrm{~nm}$. Therefore, a much sharper tip was used to reduce the minimum detectable feature size. A new Bruker Model MPP-21100-10 silicon tip with a $5 \mathrm{~nm}$ tip radius of curvature and $17.5 \mathrm{~nm}$ tip height was mounted onto the cantilever. The front and back sidewall angles are $15^{\circ}$ and $25^{\circ}$, respectively. Figure 3-6 is a picture of the AFM laboratory. The CdSe/ZnS QD substrate was mounted onto a circular metal stage using double sided tape. The microscope scanning tip is raised to provide clearance and the stage was placed onto the AFM stage under microscope tip. Using the two front screws on the AFM as a coarse adjustment, the screws were turned counterclockwise to lower the tip closer to the QD 
sample. The progression was viewed in real-time on a video screen. Veeco's Nanoscope software was used to set the scan parameters and control the instrument. WSXM scanning probe microscopy software was used to analyze the AFM results [80].

AFM images of the $\mathrm{CdSe} / \mathrm{ZnS}: \mathrm{SiO}_{2}$ quantum dot nanocomposite thin film on a quartz wafer are shown in Figure 3-7. The topography of the film reveals a uniform distribution of QDs. The white areas in the 2-D map are regions of clustered QDs. The larger particles are made up of agglomeration of smaller quantum dots, which made it challenging to determine the mean particle size. However, the particle size distribution gives an average $4 \mathrm{~nm}$ dot size. Line profiles are reported in Figure 3-8. Smaller quantum dots $\sim 2 \mathrm{~nm}$ and larger agglomerated quantum dots up to $40 \mathrm{~nm}$ were resolved. Clusters of $\mathrm{CdSe} / \mathrm{ZnS}$ quantum dots are imaged revealing the nanocomposite inhomogeneity in the $20 \times 20 \mu \mathrm{m}$ scan.

In Figure 3-9, two QDs separated by less than $50 \mathrm{~nm}$ are imaged. The height profile is shown in Figure 3-9b. Figure 3-10 charts the dot size distribution. The QD films under AFM yield an average quantum dot particle size of $3.6 \mathrm{~nm}$. EMA quantum mechanical model predicts a $3.5 \mathrm{~nm}$ QD particle size. This is in good agreement with experimental data obtained. 


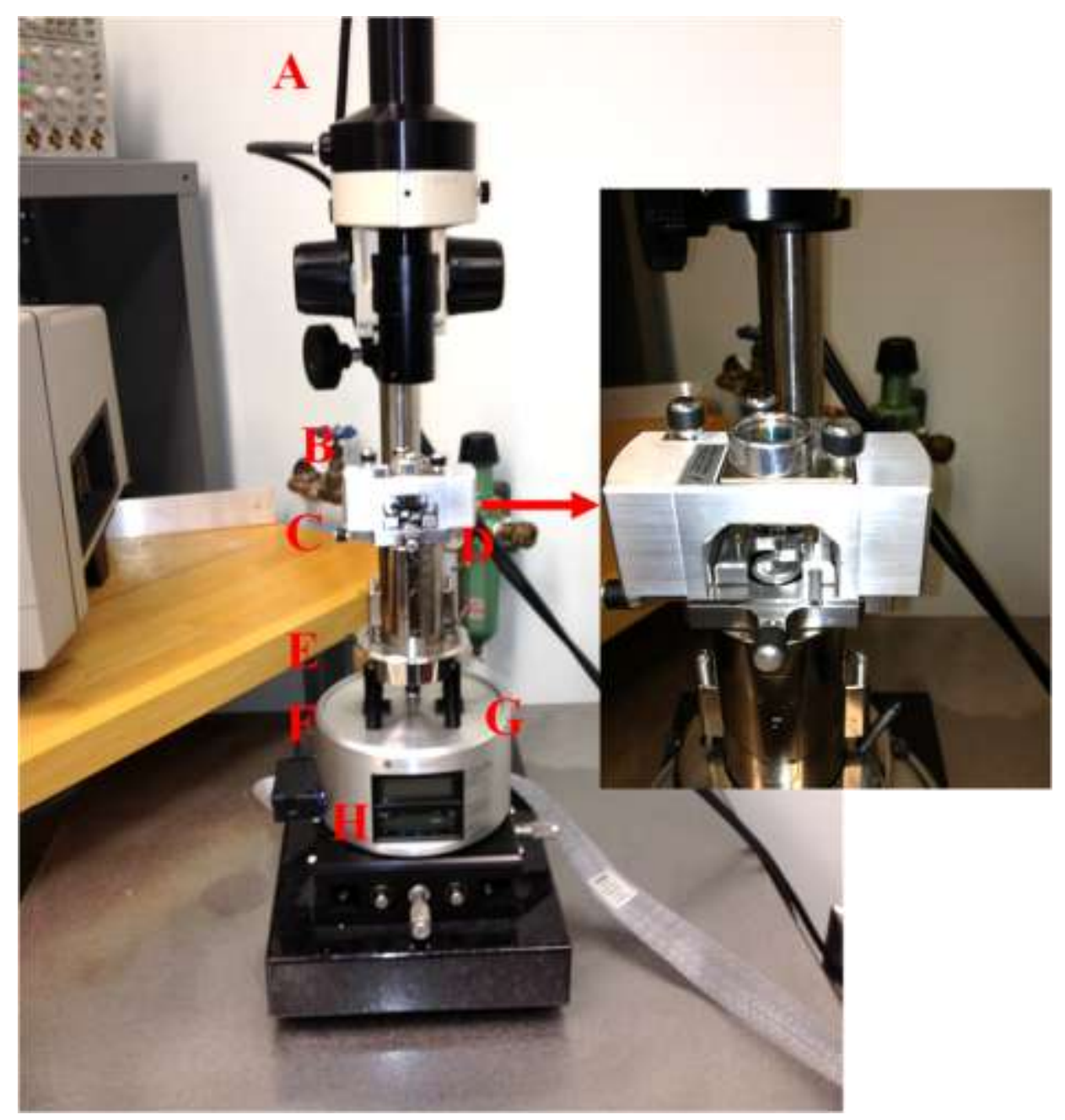

Figure 3-6: AFM. The inset is a magnified view of the QD sample mounted on the stage.
A Laser diode
B Head
C Tip
D Sample Stage
E Mode Selector Switch
F Motor Control Switch
G Base
H Digital Display 

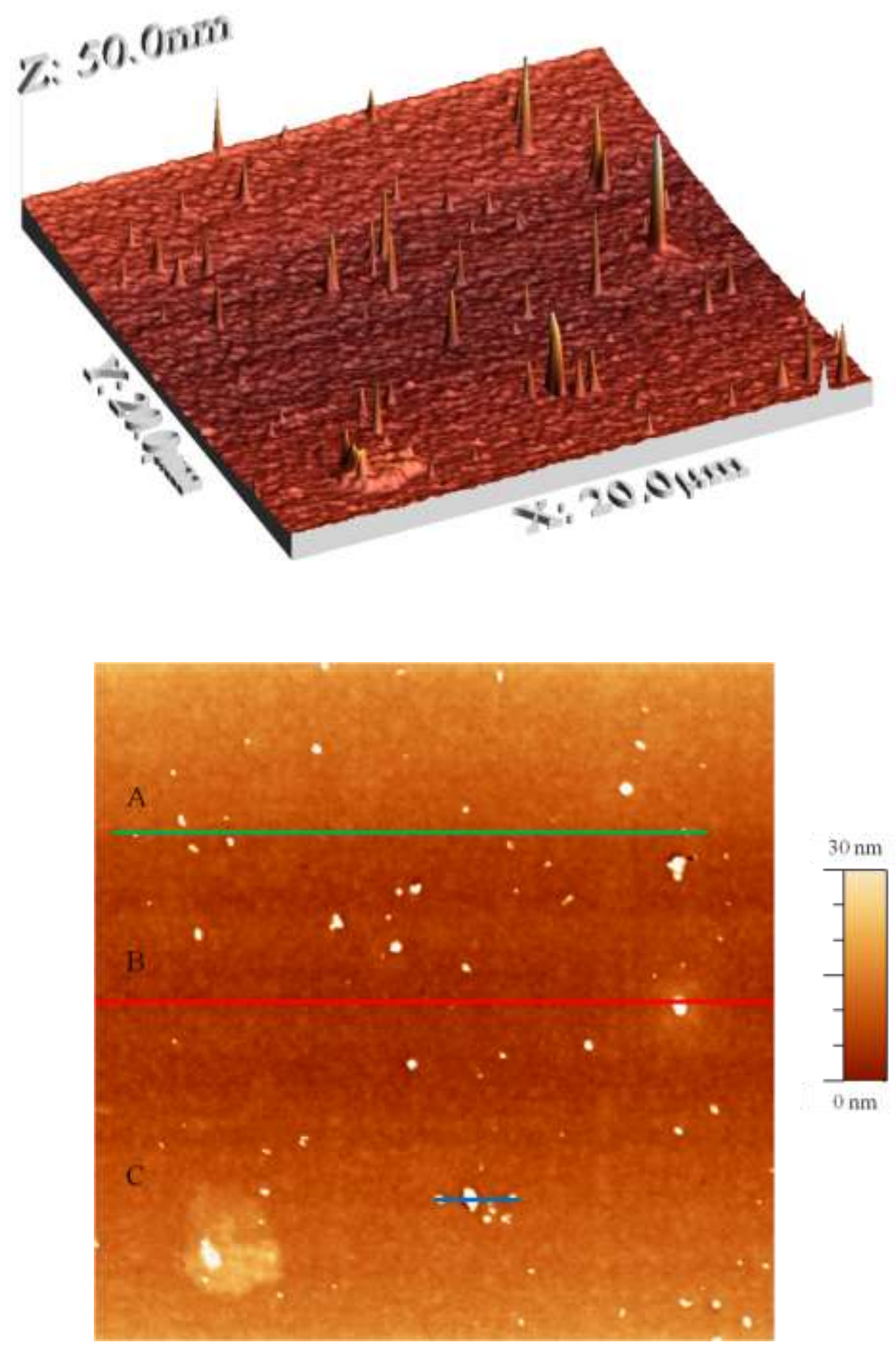

Figure 3-7: A 20 × $20 \mu \mathrm{m}$ perspective view (top image) and top view (bottom image) of $\mathrm{CdSe} / \mathrm{ZnS}: \mathrm{SiO}_{2}$ film topography. AFM line scans $\mathbf{A}, \mathbf{B}$, and $\mathbf{C}$ are plotted in the following figure. 

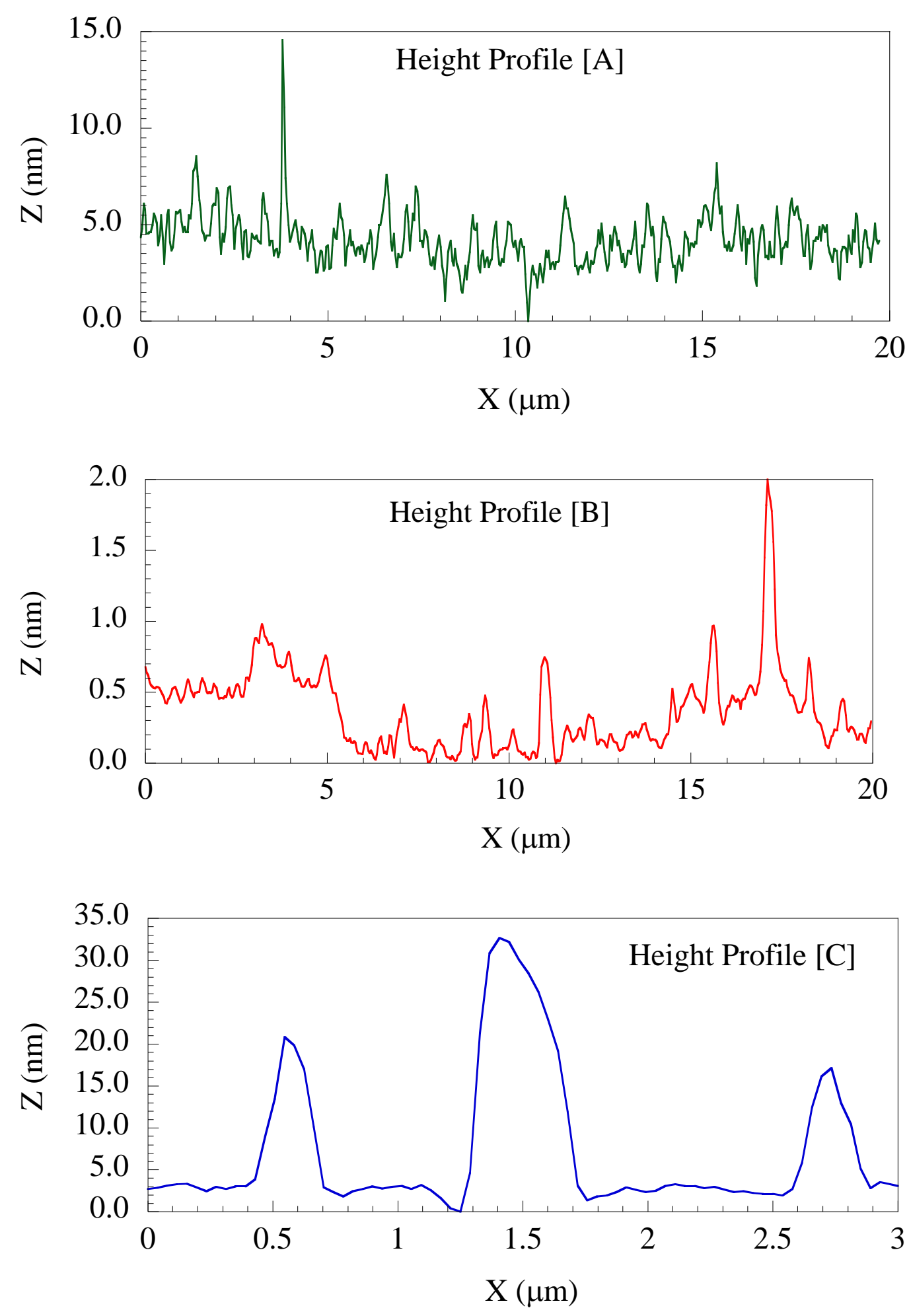

Figure 3-8: Line profile measurements of AFM image. The regions scanned to generate Height Profile [A], [B], and [C] are shown in Figure 3-7. 

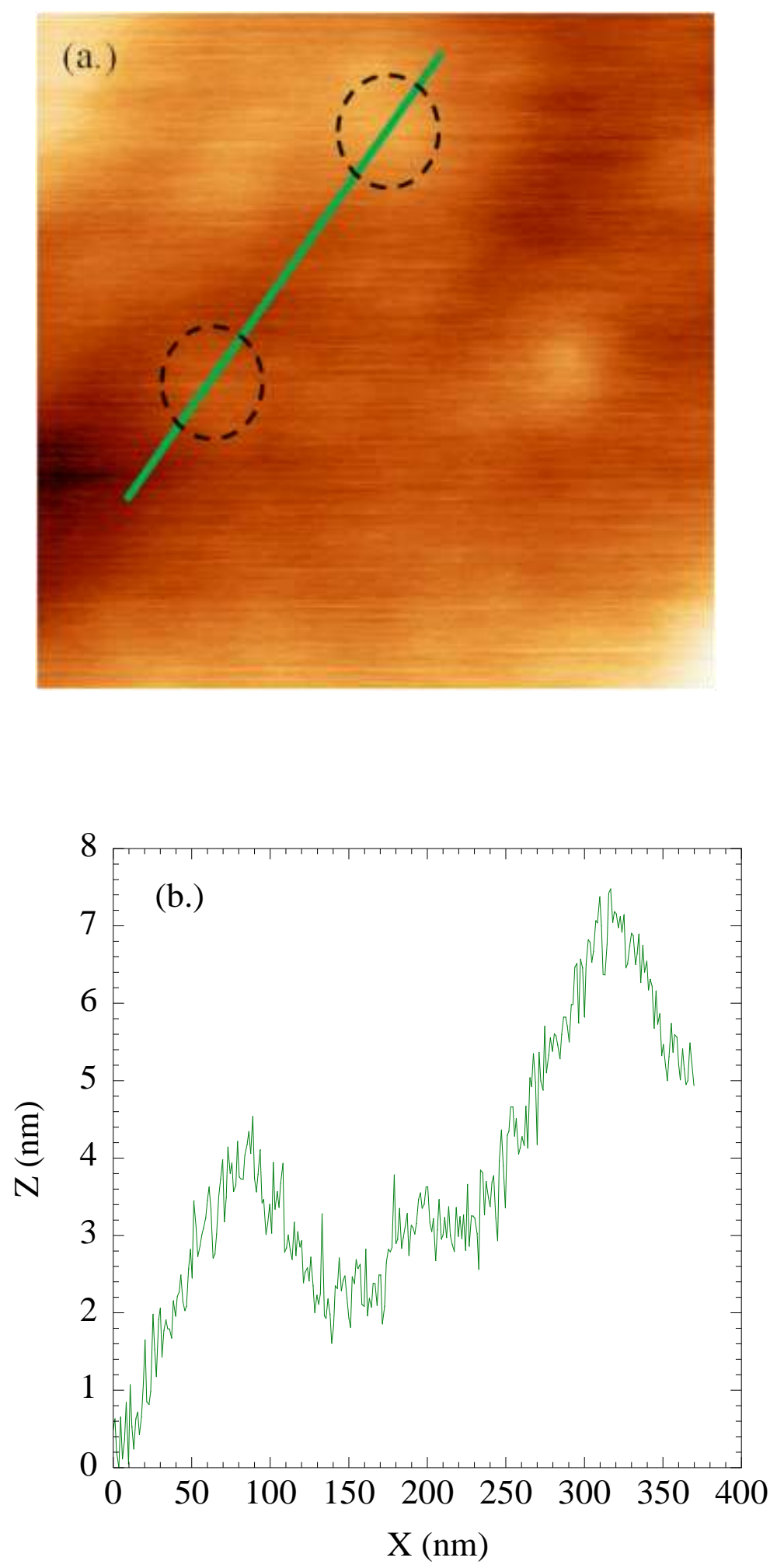

Figure 3-9: (a.) $\mathrm{CdSe} / \mathrm{ZnS}: \mathrm{SiO}_{2} \mathrm{AFM}$ image and (b.) height profile of individual QDs. 


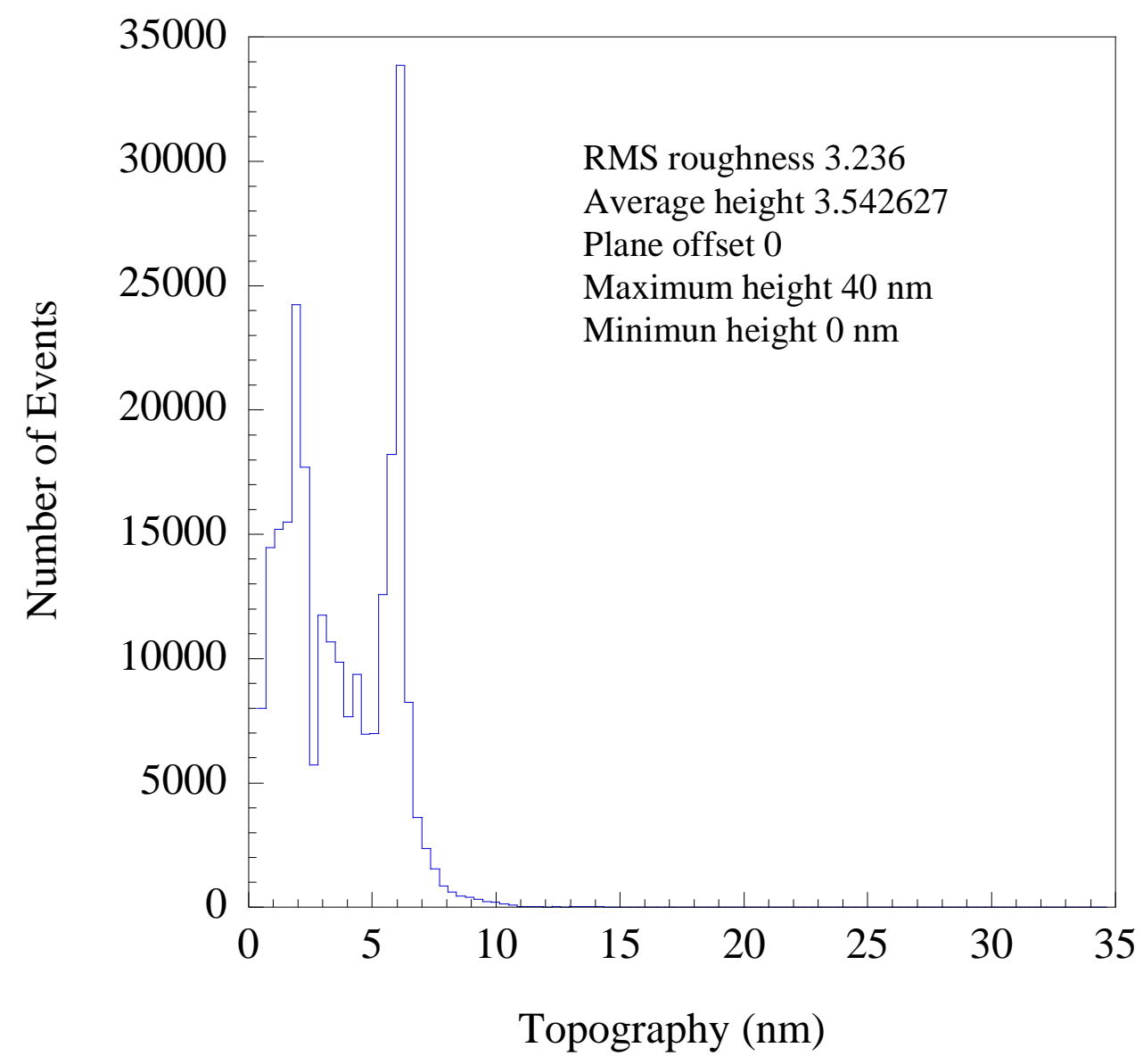

Figure 3-10: QD size distribution obtained from several AFM images. 


\section{Chapter 4 QUANTUM DOT LUMINESCENCE THERMOMETRY}

"A scientist in his laboratory is not only a technician: he is also a child placed before natural phenomena which impress him like a fairy tale."

Marie Curie

This chapter presents experimental apparatus, laboratory procedures, and calibration reports for luminescence thermometry. The details on the experimental setups that were designed and built for quantum dot luminescence thermometry are provided.

\subsection{Optical Absorption Measurement System}

A Perkin Elmer Lambda 900 UV/Vis/NIR spectrophotometer was used for the absorption measurements. The Lambda 900 incorporates two juxtaposed monochromators. Each monochromator is equipped with two gratings and two detectors: a photomultiplier tube (PMT), and a lead sulfide ( $\mathrm{PbS})$ detector. A deuterium lamp and a halogen lamp covered the working wavelength range, 175-3300 $\mathrm{nm}$ of the spectrometer. The instrument resolution is $0.05 \mathrm{~nm}$ in the UV/Vis and $0.20 \mathrm{~nm} \mathrm{NIR}$. The UV WinLab software allowed fully automated control of scan input parameters and instrument optics. The spectrophotometer workstation and a schematic of the double beam optical system layout are shown in Figures 4-1 and 4-2, respectively. 


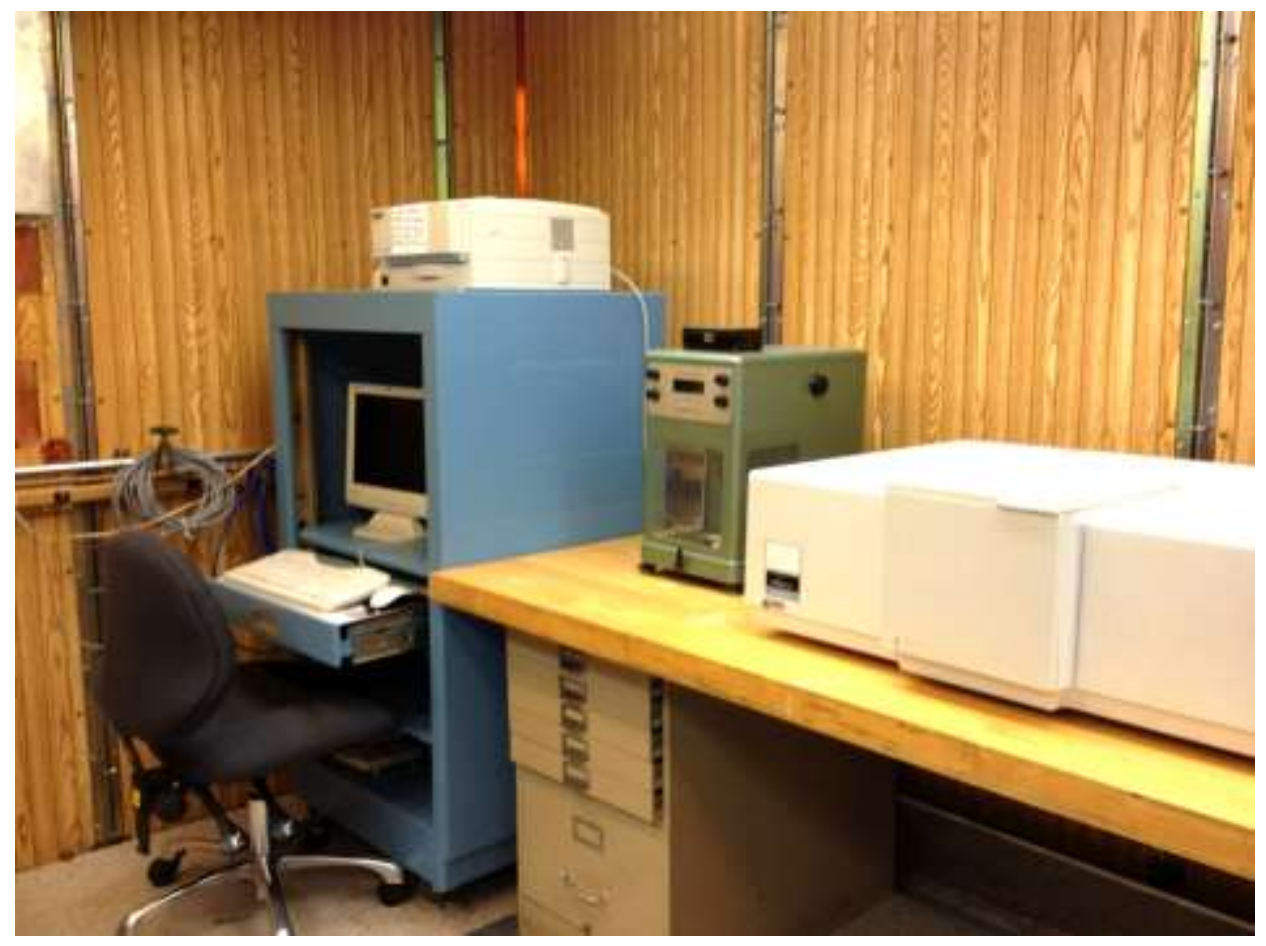

Figure 4-1: Absorption spectroscopy workstation. 


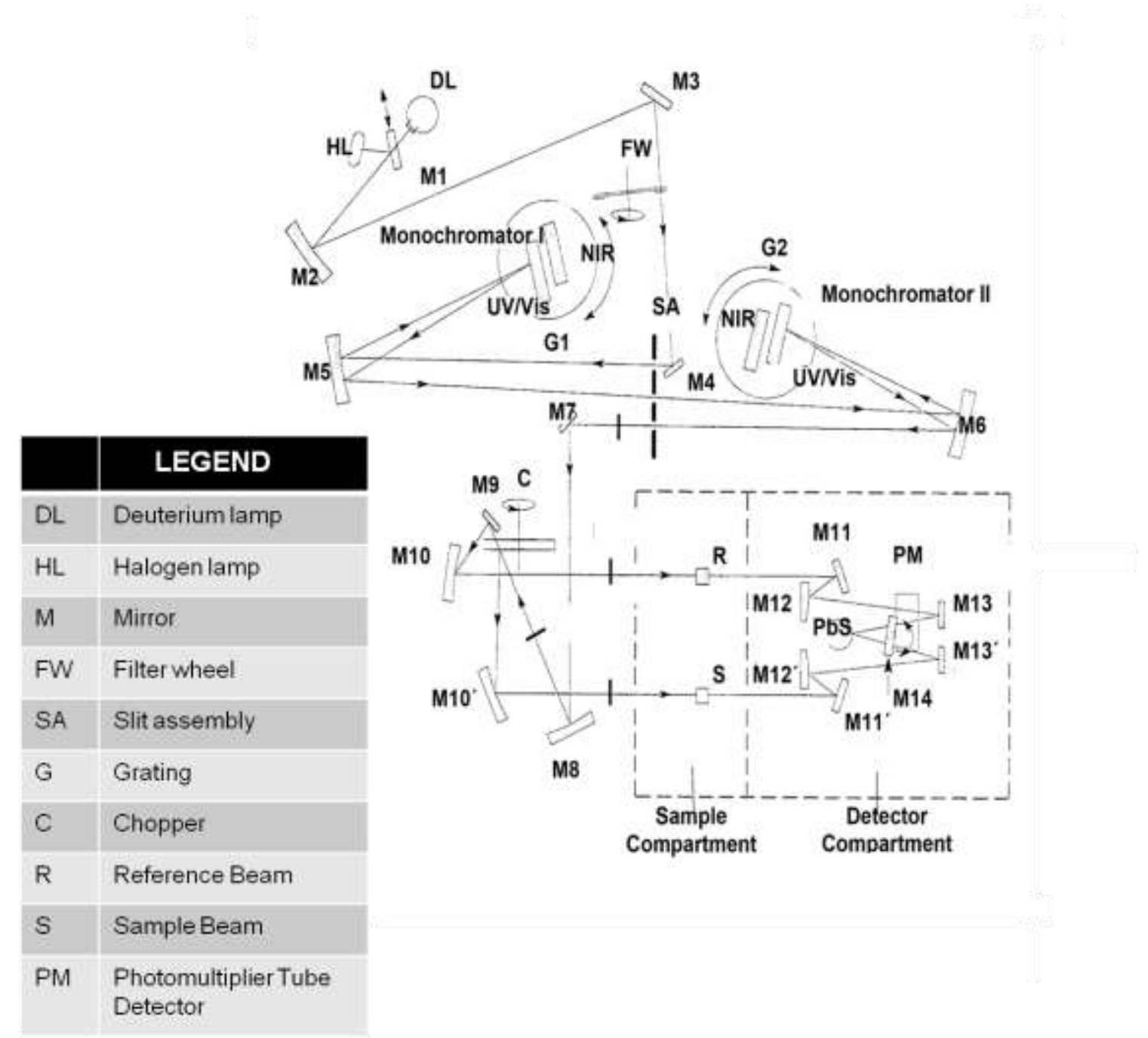

Figure 4-2: Double beam spectrometer optical layout [81]. 
Two $1.27 \mathrm{~cm}$ cuvettes and the cuvette-holder sampling accessory were mounted inside the sample chamber for absorption measurements on the fabricated quantum dot solutions. The quantum dot solutions were dispensed with a dropper into disposable specialty cuvettes manufactured by BrandTech. The BrandTech semi-micro cuvettes are UV-transparent and chemical resistant. An empty cuvette was positioned in the reference beam path and the cuvette with the QD-solution was inserted into the optical signal beam pathway.

The quantum dot thin films were mounted in the sample chamber as shown in Figure 4-3. Fig. 4-3 is viewed from the top of the sample compartment. The QD film sample holder was fabricated in-house. It consists of a double-ring aluminum mount with a 1/16 inch set screw used to secure the quartz substrate and aperture. The outer ring held the QD substrate and the inner ring held the aperture in place. Alumina (96\% $\mathrm{Al}_{2} \mathrm{O}_{3}$ ) flat disk apertures were custom fabricated and purchased from Stellar Industries Corporation. All disk apertures were $25.4 \mathrm{~mm}$ in diameter and $0.64 \mathrm{~mm}$ thick. The aperture sizes range from $2-4 \mathrm{~mm}$. The base of the sample holder was ground flat such that the mount would sit level on the heating plate. A Waltlow Ultramic 600 thin film heating element was purchased. The heater was a 2.54 x $2.54 \mathrm{~cm}$ square plate constructed of aluminum nitride (AIN). The AlN heater had a $650 \mathrm{~K}$ maximum operating temperature value. The temperature was controlled using a Watlow EZ-Zone programmable temperature controller with ramp and soak profile. The components labeled in Fig. 4-3 are clearly shown in Figure 4-4. The quantum dot thin film sample mount, reference beam mount, apertures, heating element, temperature controller, and optical stages are pictured. 


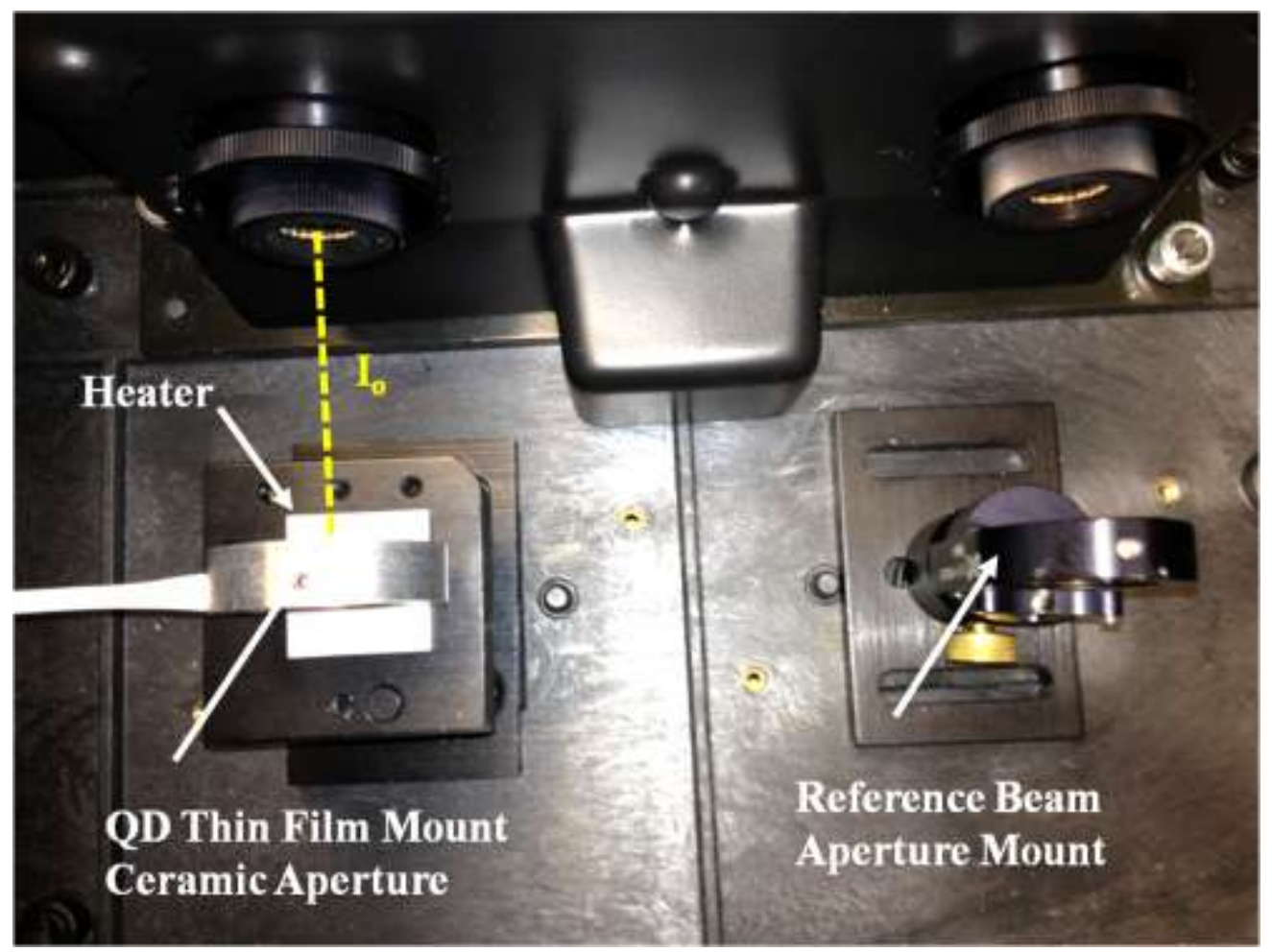

Figure 4-3: Quantum dot thin films mounted in the spectrometer sample chamber. 


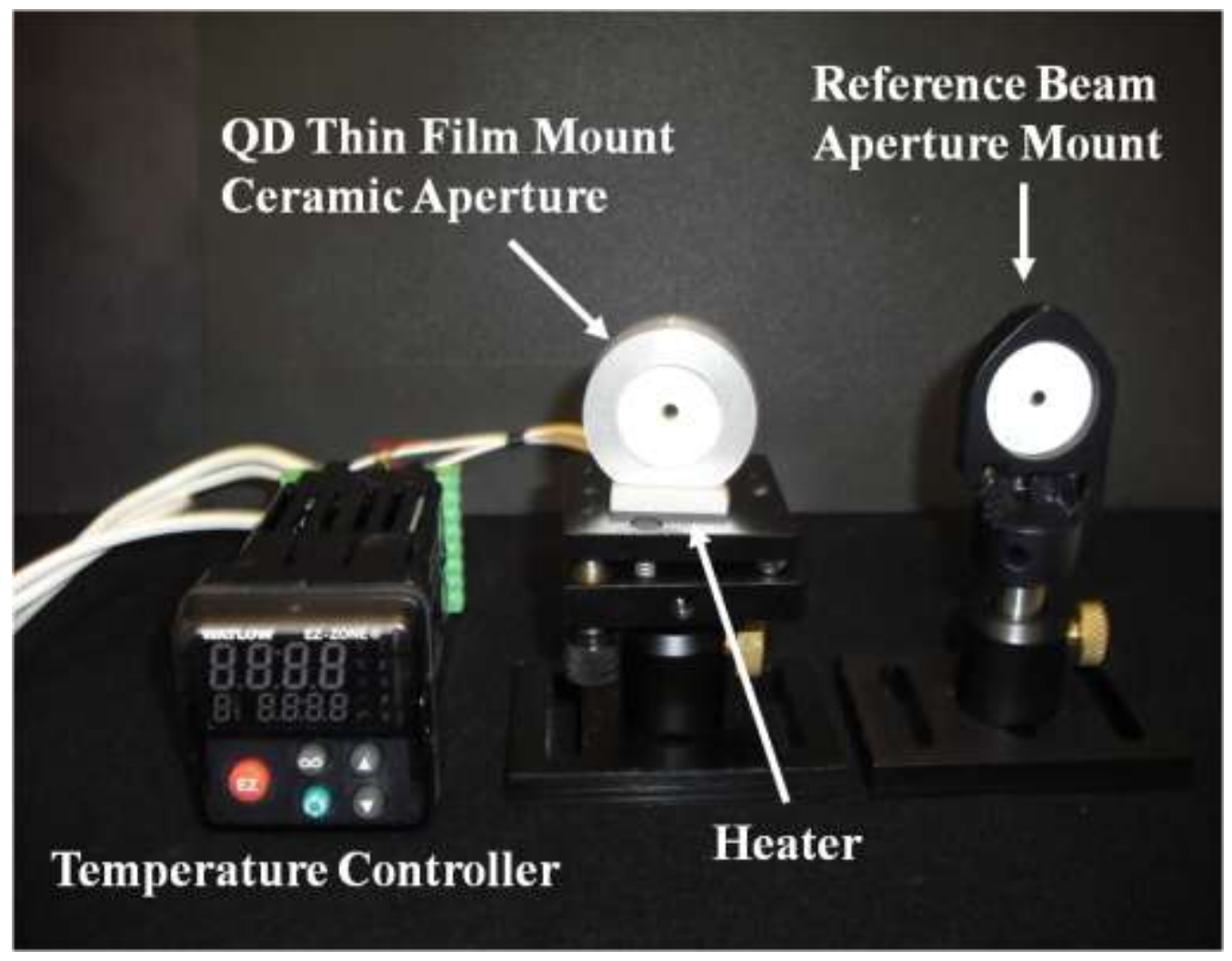

Figure 4-4: Temperature controller, sample mount, heater, and ceramic apertures. 
Temperature dependent absorption on select bulk crystals were investigated and the results are recorded in Appendix C. These measurements were carried out using the heating element described above. The crystals were placed directly onto the AIN heater which was mounted onto an optical stage. The crystals were aligned in the beam path with the orientation axis of interest perpendicular to the optical path of the radiation source. For example, when measuring the optical transmission along the c-axis of biaxial alexandrite, $\mathrm{BeAl}_{2} \mathrm{O}_{4}: \mathrm{Cr}^{3+}$ (reference Appendix C), the crystal was placed on the sample stage with the c-axis perpendicular to the beam.

\subsection{Photoluminescence Measurement System}

Quantum dot thin film photoluminescence (PL) measurements were carried out using the equipment pictured in Figure 4-5. This experimental set-up was designed and built for steady-state luminescence thermometry on quantum dot thin films. Figure 4-6 is a schematic of the experimental apparatus labeled in Fig. 4-5. An Omnichrome Model 532-AP diode pumped solid state laser powered by an Omnichrome Model 150 power supply was used as the excitation source. The laser was operated at $532 \mathrm{~nm}$ and had a $500 \mathrm{~mW}$ maximum output power. The sample was excited at $\sim 45^{\circ}$ with respect to normal of the film. A one-to-one imaging technique was implemented for PL signal collection. A $5.00 \mathrm{~cm}$ focal length lens was used to collect the signal and focus it into a $600 \mu \mathrm{m}$ optical collection fiber. The opposite end-tip of the collection fiber had a cylindrical end-tip with a $0.75 \times 0.05 \mathrm{~cm}$ slit. The signal was modulated by a chopper placed at the entrance to a monochromator. The Stanford Research Systems, Incorporated optical chopper system included a 4 inch diameter, 5/6 slot chopper wheel 
and a model SR540 chopper controller. An RG580 long pass glass colored filter blocked the pump beam. A $11.43 \mathrm{~cm}$ focal length lens was used to focus the luminescence into a 500M SPEX spectrometer. A $1200 \mathrm{gr}-\mathrm{mm}^{-1}$ grating blazed at $1.2 \mu \mathrm{m}$ wavelength was used in the spectrometer. A $0.10 \mathrm{~nm}$ resolution was obtained with a $50 \mu \mathrm{m}$ and $100 \mu \mathrm{m}$ spectrometer slit width. An Oriel model 70685 side-on photomultiplier tube (PMT) detected the signal. The PMT was driven at $1200 \mathrm{~V}$ supplied by a Model 3000R EMI Gencom Incorporated high voltage power supply. The chopper operating at $150 \mathrm{~Hz}$ provided the reference signal to a Stanford Research System Model 530 lock-in amplifier. Data storage and processing were carried out using the $\mathrm{Cd} 2 \mathrm{~A}$ data acquisition computer program. 


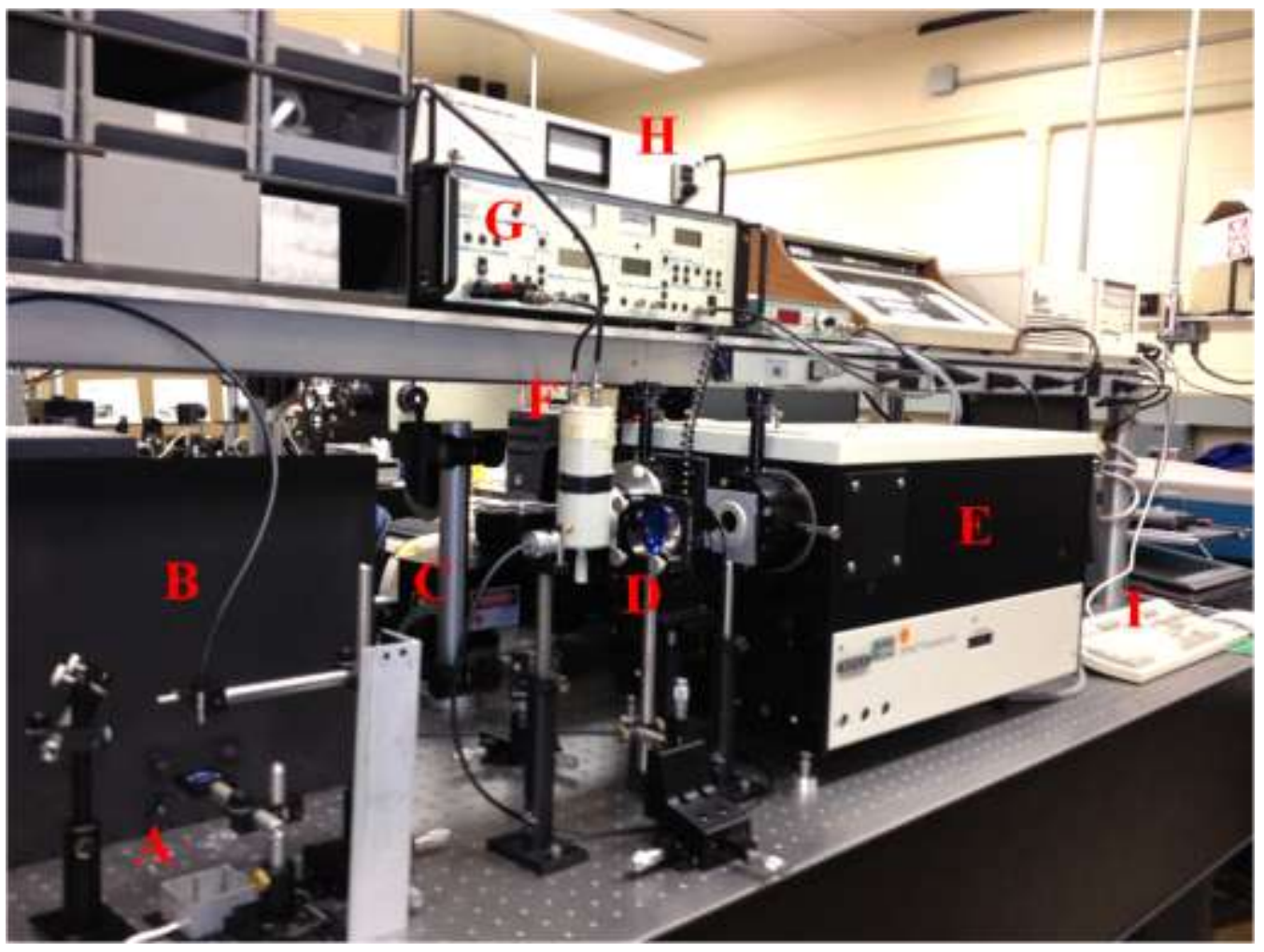

Figure 4-5: Quantum Dot Luminescence Thermometry Laboratory.

A Sample Mount

B Optical Fiber

C Laser

D Collection Lens and Optical Chopper

E Spectrometer

F PMT

G Lock-in Amplifier

H Power Supply

I Computer 


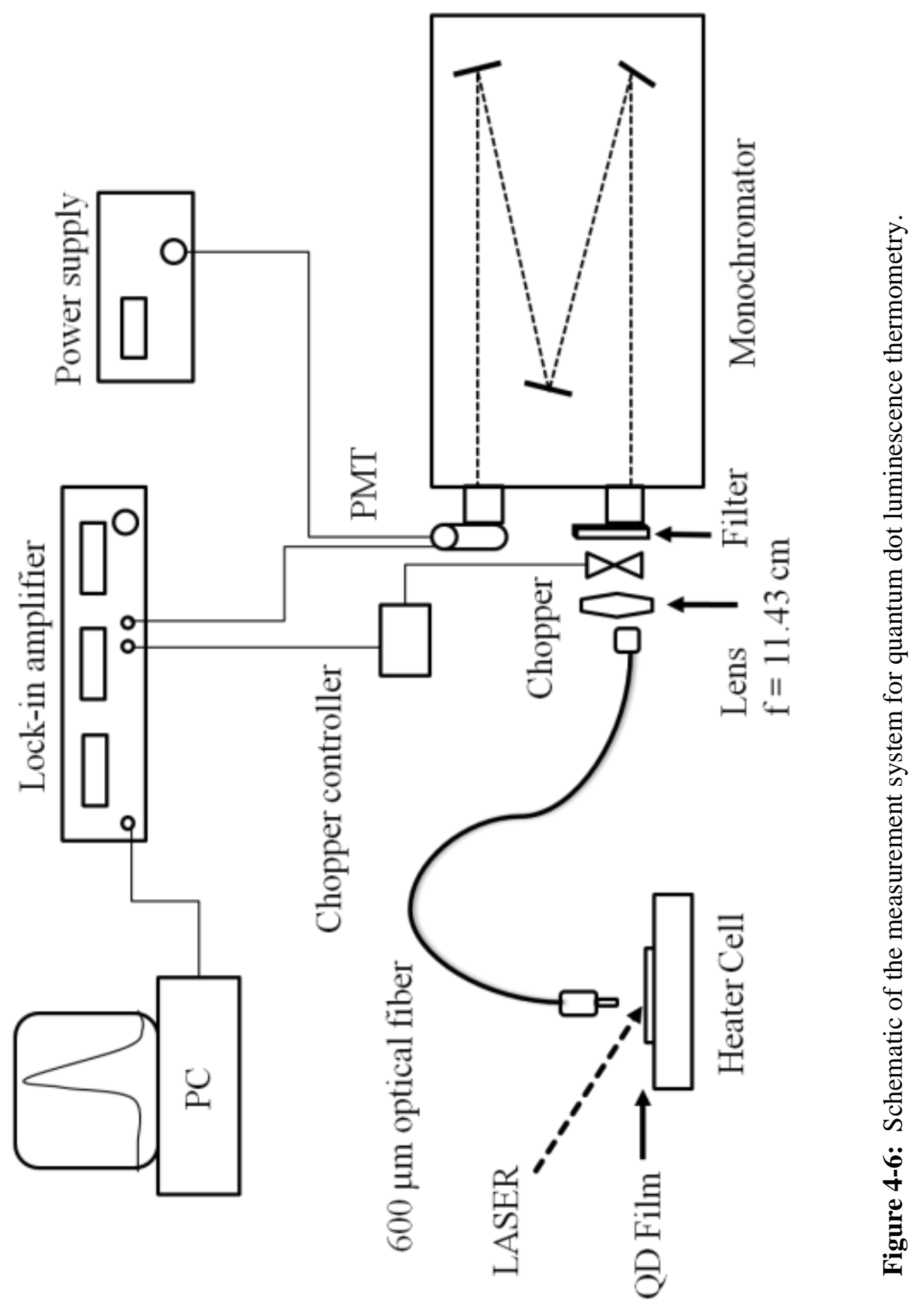


The SPEX monochromator was calibrated using a Mercury (Hg) lamp. The $\mathrm{Hg}$ lamp and a collection lens were inserted just outside of the spectrometer entrance in front of the chopper. Approximately $20 \mathrm{Hg}$-lamp emission lines ranging from 3600 to

$11000 \AA$ were chosen from the calibration standards table. The monochromator scans were taken within $\pm 5 \AA$ of the expected peak and the measured emission line was recorded. $\mathrm{A} \sim 0.10 \mathrm{~nm}$ resolution was obtained with a $50 \mu \mathrm{m}$ and $100 \mu \mathrm{m}$ slit width. The calibration curve is plotted in Figure 4-7. 


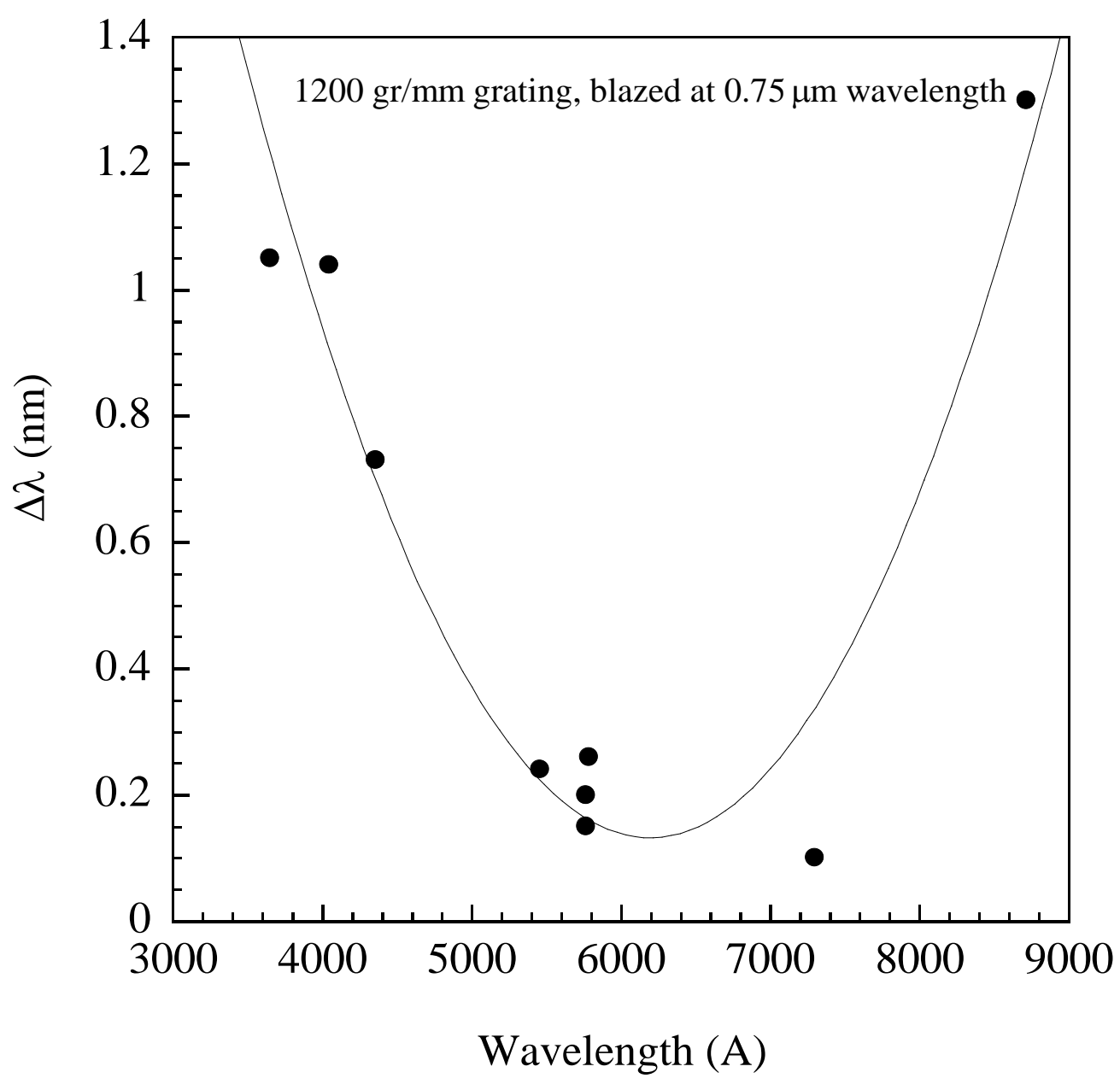

Figure 4-7: SPEX 500-M monochromator calibration plot. 
Temperature-dependent QD emission spectra up to $550 \mathrm{~K}$ were acquired by incorporating a heating element and heat-bath cell assembly into the QD luminescence experimental setup. The heat-bath cell prevented air draft and maintained a uniform temperature distribution on the quantum dot thin film. The heat-bath cell was designed and constructed in-house. The aluminum cell dimensions were 5.08 x 5.08 x $2.54 \mathrm{~cm}$. The top of the cell consisted of transparent glass. The inside of the cell was painted black to reduce laser beam scatter. The samples were mounted on the heater plate and enclosed with the heat-bath cell. The same heating element (Watlow Ultramic 600 ceramic plate) that was used for the absorption spectroscopy was incorporated into the PL thermometry measurement system. The heater was secured to a machinable ceramic (macor) base plate. The metal mounting screws were isolated from the heating element by inserting thin ceramic strips between the screw-head and the heater. A picture of the cell assembly that surrounds the heater is shown in Figure 4-8a. A schematic of the aluminum nitride (AlN) heater and macor stage mount is shown in Figure 4-8b. 


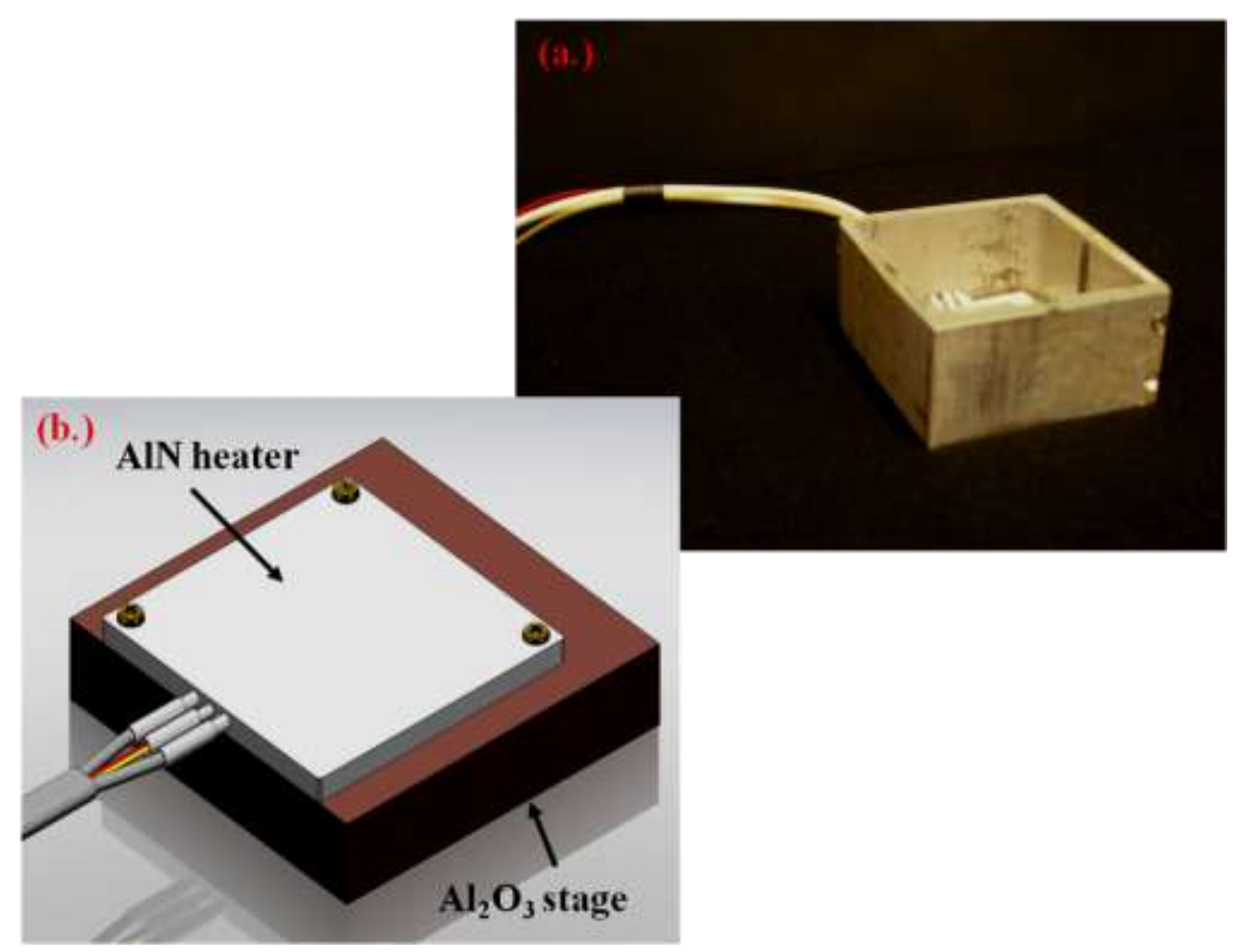

Figure 4-8: (a.) Heater cell surrounds the heating element with leads to the temperature controller shown and (b.) A schematic of the aluminum nitride (AlN) heater and macor stage located inside the heater cell. 
Extension of QD luminescence thermometry into ultra-high temperature regime $(\sim 650 \mathrm{~K})$ was accomplished by using a custom designed high-temperature heating system. A microheater, BPAN-O-2010 control unit, transformer, and thermocouple (TC) were purchased as a high temperature heating elements package from Micropyretics Heaters International (MHI) Incorporated. First, the control unit and transformer were hard wired. Then a high temperature resistant stage was designed and built for the microheater and quantum dot sample. The unit was then tested and calibrated for controlled heating extending to $\sim 650 \mathrm{~K}$.

The primary power $(120 \mathrm{~V})$ was connected to the BPAN-O control panel. The transformer was used to step down to the heater voltage. Two high-temperature secondary wires (16 gauge with fiberglass jacket) connected the transformer to the heater. A schematic of the closed loop control system is shown in Figure 4-9. The MHI microheater was a freely radiating disk. It consisted of 1.5 inch spiral center ceramic heating element and two 2 inch leads extending from either side. Two aluminum hightemperature braided wires and two terminal blocks (lugs) were supplied with the heater. The braided wire and lugs were used to mount the heating element. The braided wire had a loop with a bolt at one end and the other end was free. The bolted end connected the heater leads and the free end connected the terminal blocks. The terminal blocks isolated the heater from the secondary wires off the transformer. This prevented excess stress on the microheater. The wires were connected to the terminal blocks as shown in Figure 4-10.

Two macor fractalin boards were purchased from Mattech Corporation. One board ( $4 \times 4 \times 0.5$ in.) was used to mount the lugs and the microheater. The second board 
$(12 \times 12 \times 0.5$ in.) was used to isolate the two secondary wires from the optical table. Both boards were $99 \% \mathrm{Al}_{2} \mathrm{O}_{3}$ and were manufactured-specified to support a maximum use temperature of $1470 \mathrm{~K}\left(1200^{\circ} \mathrm{C}\right)$. The microheater and sample stage are shown in Figure 4-11.

Calibration reports were generated for the heater. The $\%$ output power versus current from the controller is plotted in Figure 4-12. For the high temperature range $50 \mathrm{~A}$ maximum current was required through the wires. This was found to be $\sim 80 \%$ output power. The unit was set to $80 \%$ output power during the tests. The temperature on the disk was monitored as a function of the thermocouple (T.C.) readout. Temperature versus T.C. output is reported in Figure 4-13. 


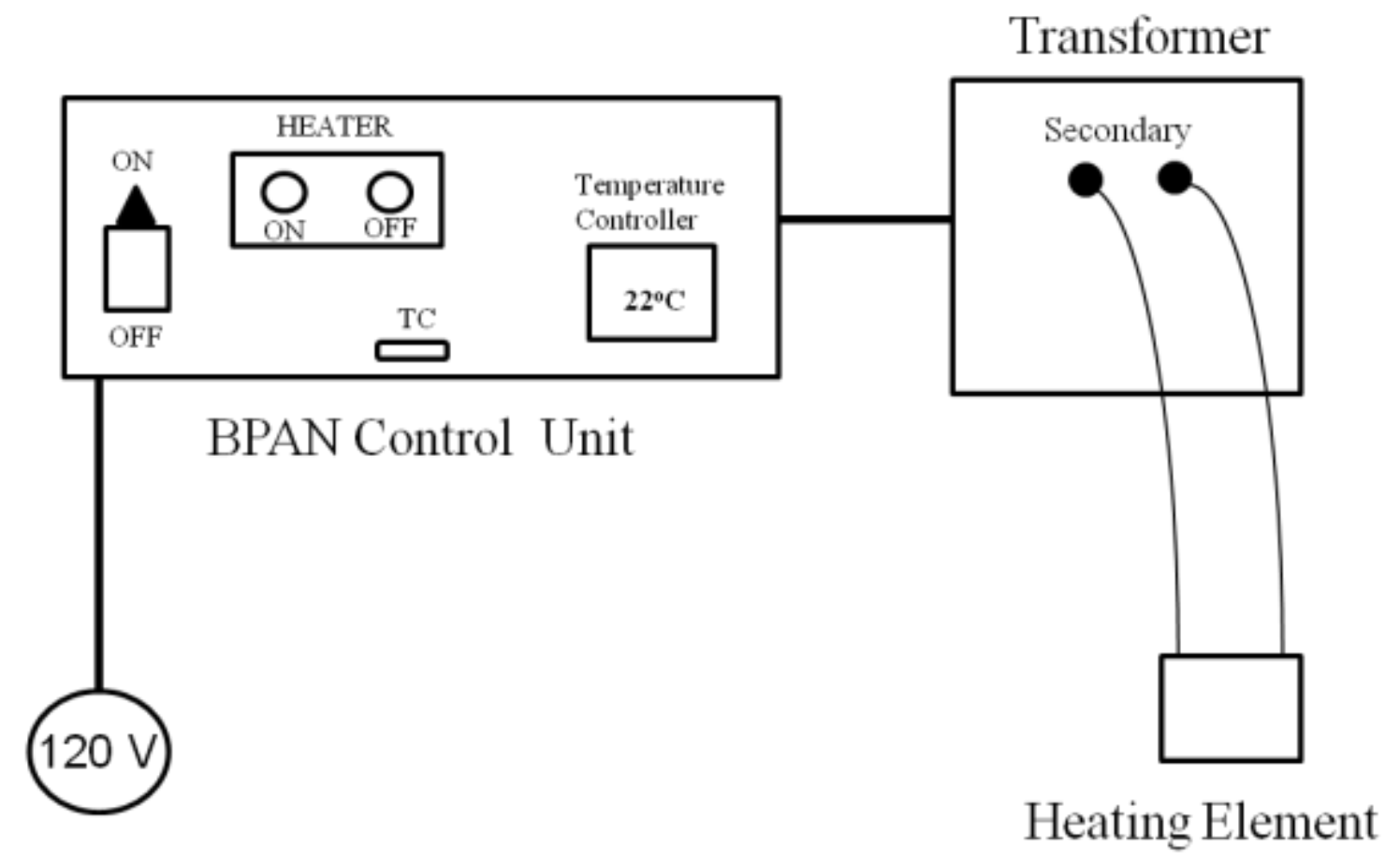

Figure 4-9: Microheater system electrical flow diagram. 


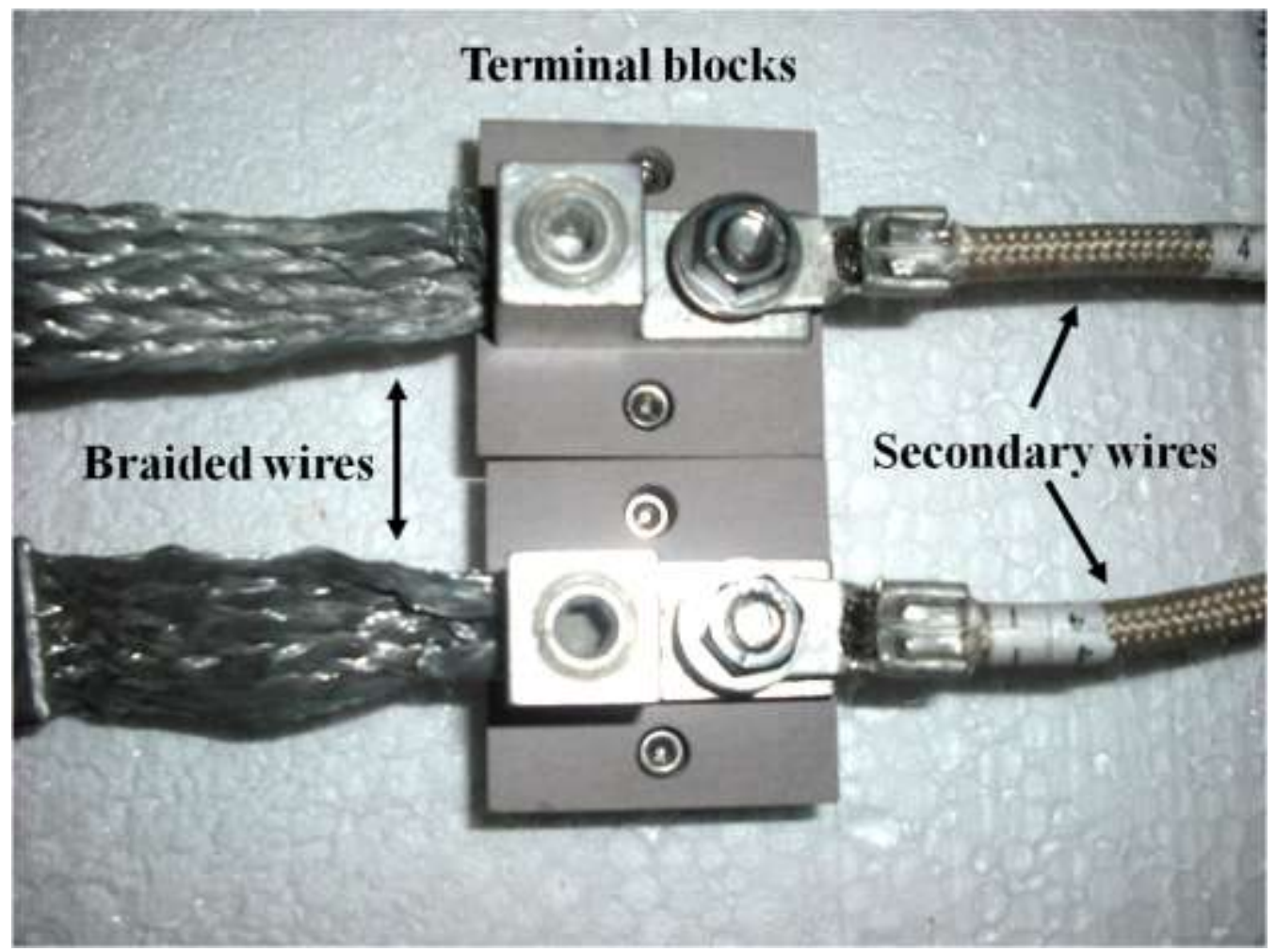

Figure 4-10: Copper terminal leads secure the secondary wires to the terminal blocks. Round-base lug screws hold the braided wires in place. The terminal blocks are screwed onto a macor base plate. 


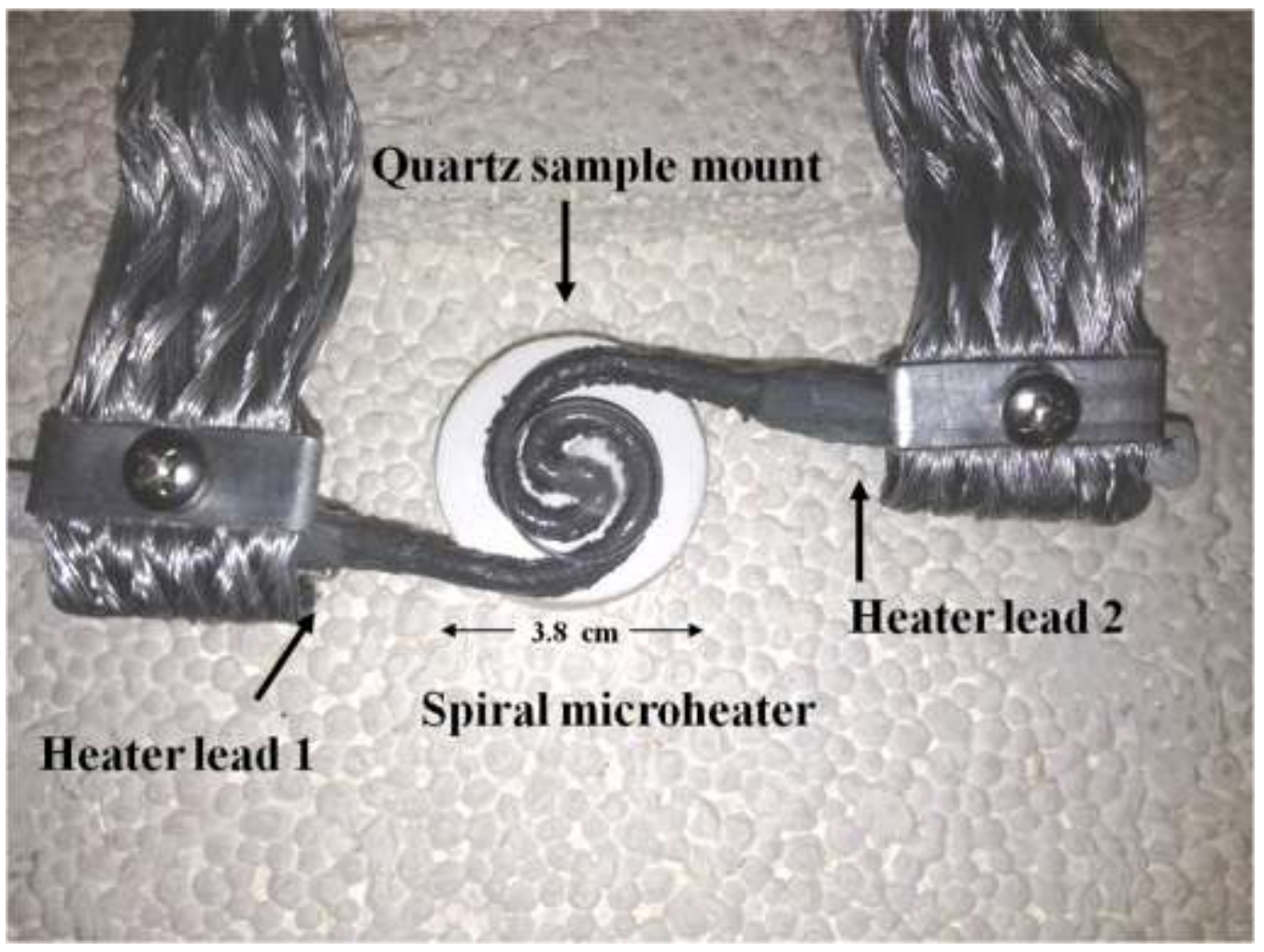

Figure 4-11: Spiral microheater and sample stage. 


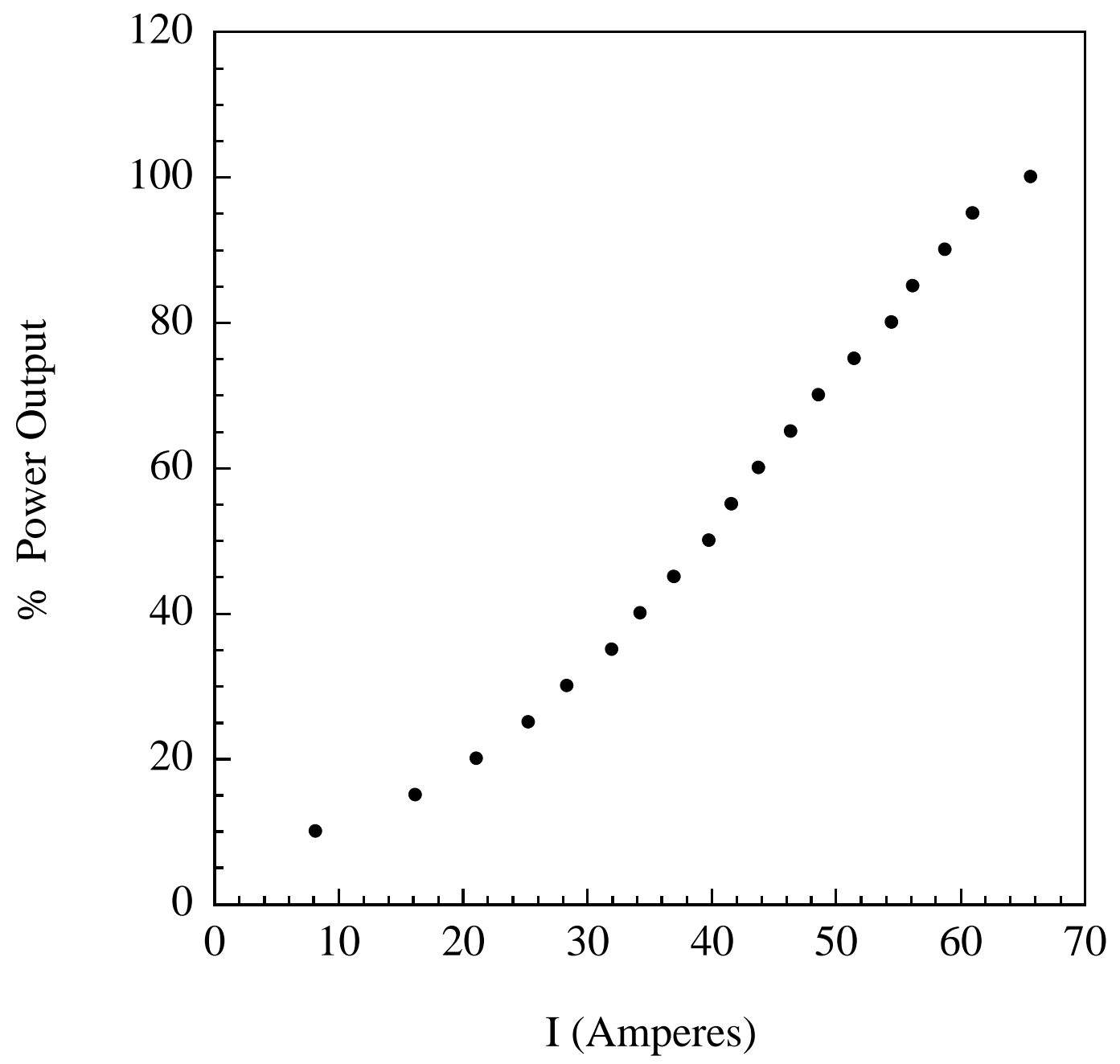

Figure 4-12: \% Power output versus current from the B-PAN control unit. 


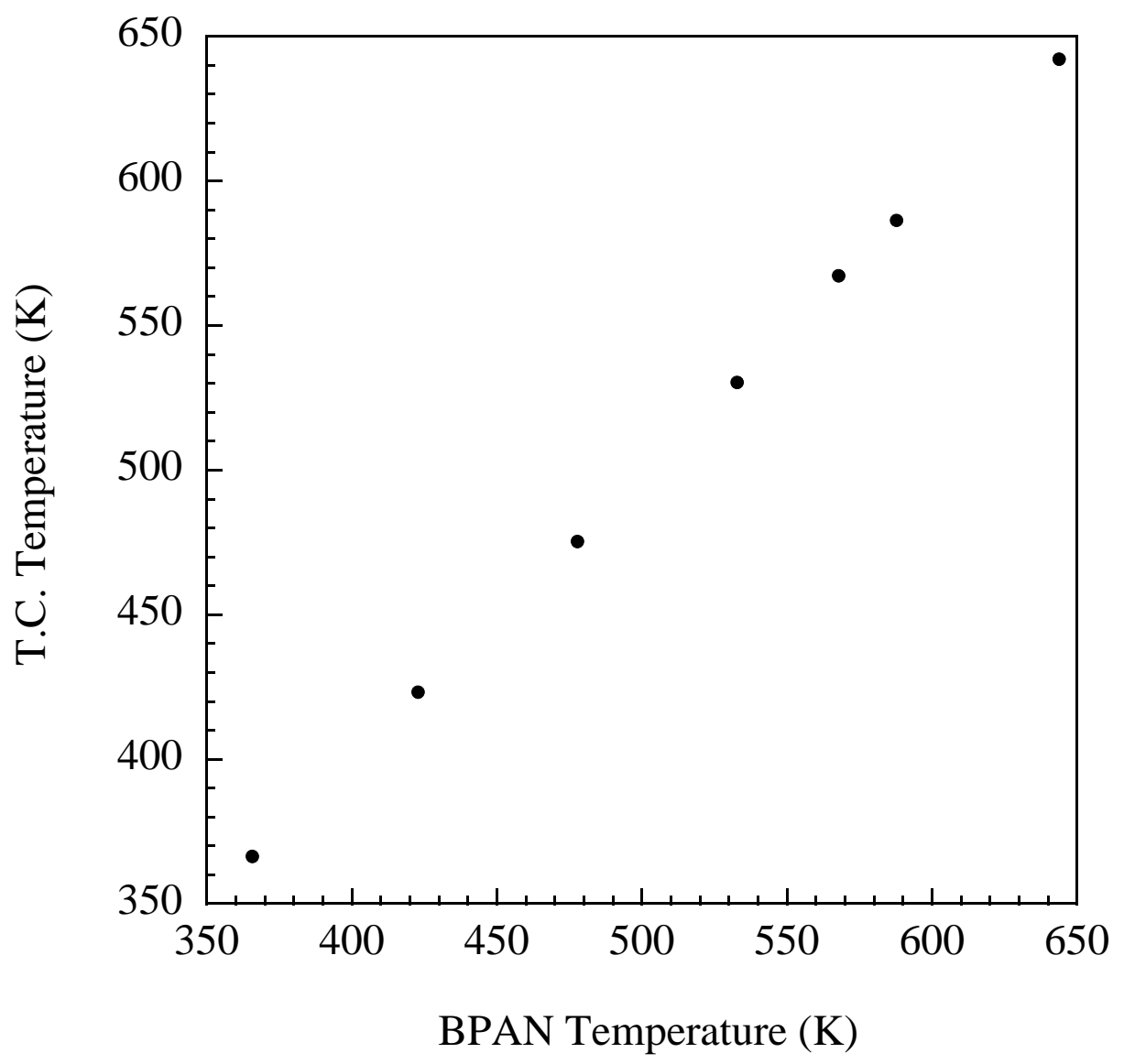

Figure 4-13: Thermocouple temperature reading versus B-PAN control unit temperature. 


\subsection{Lifetime Measurement}

Temperature dependent time-resolved luminescence on bulk crystal alexandrite was investigated. The complete study is presented in a paper attached in Appendix C. The lifetime measurement system consisted of an excitation source, an optical collection system, and an amplification and detection system. The main system components specifically designated for lifetime measurements included a pulsed laser, optical lenses, filters, a monochromator, and a PMT. A detector and an oscilloscope captured, amplified, and stored the signal. An Opotek Vibrant LD 355II pulsed laser was wavelength tuned by adjusting the crystal in the laser cavity. Opotek control software V1.090 provided fully automated control of wavelength selection and pulse duration. The signal off the PMT was feed into an oscilloscope via a standard BNC connection for processing and data storage. 


\section{Chapter 5 \\ THERMOMETRY USING QUANTUM DOT CdSe/ZnS:PMMA NANOCOMPOSITE}

"Science investigates; religion interprets. Science gives man knowledge which is power; religion gives man wisdom which is control."

Martin Luther King Jr.

\subsection{Temperature Dependent Absorption Spectroscopy}

UV-vis transmission spectra on CdSe/ZnS:PMMA thin films were recorded using the Perkin-Elmer Lambda 900 spectrophotometer described in Chapter 4. The samples were mounted into the sample compartment as explained in Section 4.1. The room temperature CdSe/ZnS:PMMA quantum dot optical absorption spectrum is shown in Figure 5-1a. The transitions in the $\mathrm{CdSe} / \mathrm{ZnS}$ quantum dot are defined by the optical absorption spectrum peaks. Shown in Figure 5-1b, the peaks correspond to transitions between discrete electronic states within the valence band and the conduction band. The exciton energy levels lie in the low-energy region of the optical absorption spectrum. The first exciton absorption band is located at a peak wavelength of $614 \mathrm{~nm}(2.02 \mathrm{eV})$. The bound electron-hole pairs extend over several unit cells of the hexagonal wurtzite crystal structure. The hexagonal wurtzite CdSe structure has a $9.6 \mathrm{~nm}$ Bohr radius. Weaker Wannier-Mott exciton interactions are observed at higher energies in the room temperature measurement. 


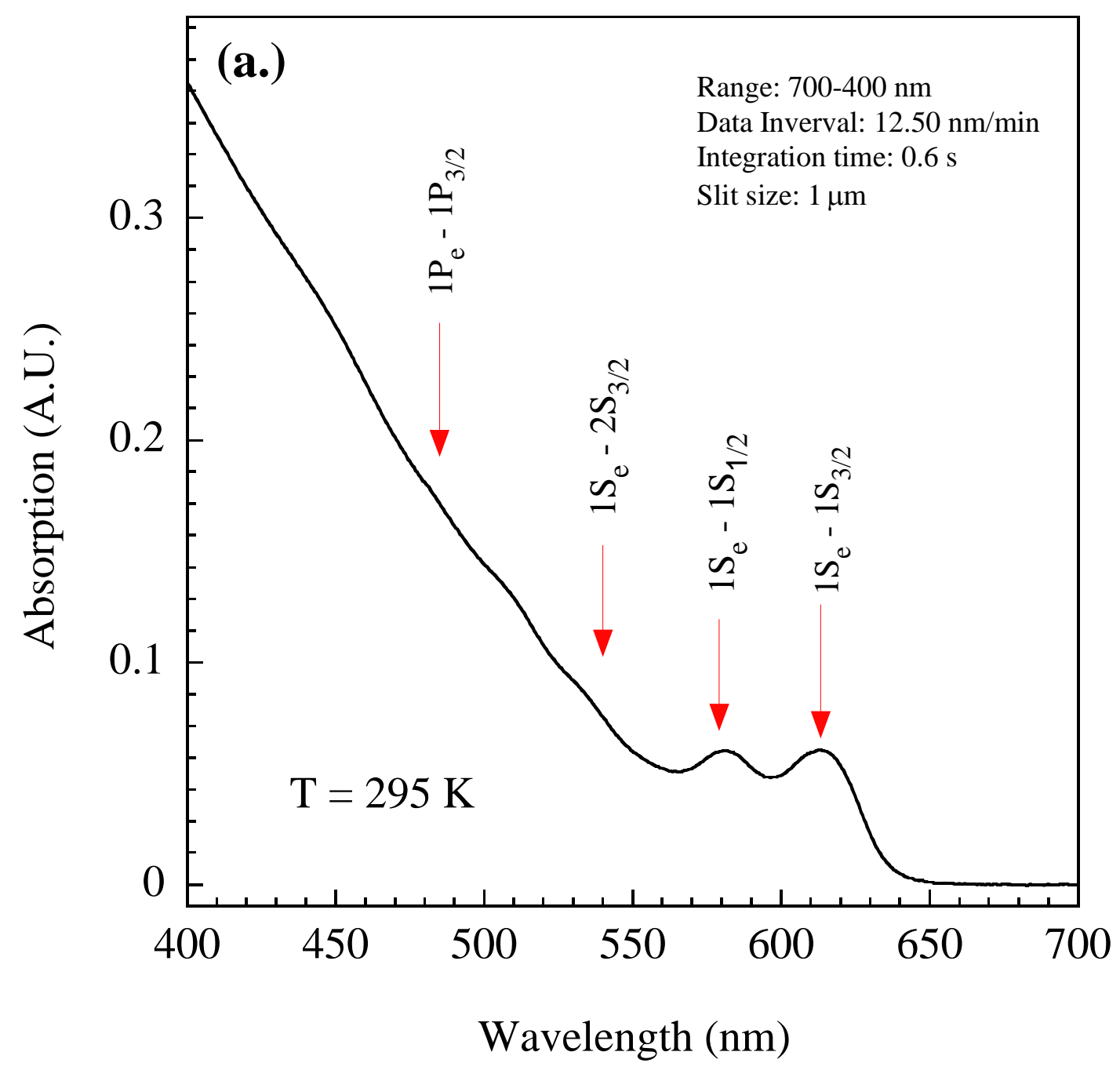


(b.)

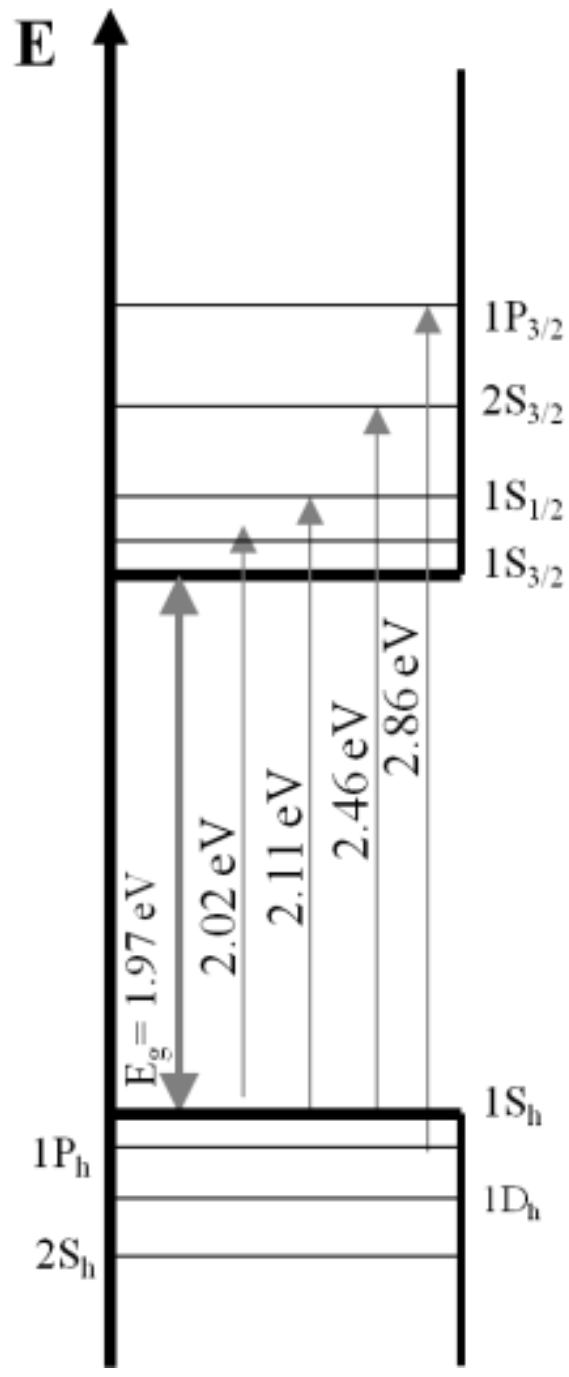

Figure 5-1: (a.) Room temperature CdSe/ZnS:PMMA nanocomposite absorption spectrum. (b.) Energy level diagram of the optical absorption transitions in $\mathrm{CdSe} / \mathrm{ZnS}$ quantum dots. The energy values were extracted from (a.). 
It was possible to resolve a good deal of fine structure in the CdSe/ZnS:PMMA nanocomposite. This is a strong indicator of a uniform QD size distribution. The fine structure was resolved using a convolution of a Lorentzian homogeneous line profile with an inhomogeneous lineshape function given by:

$$
\begin{aligned}
& f(v)=f_{L}(v) \otimes f_{G}(v)=\frac{\alpha_{L}}{\pi} \frac{1}{\left(v-v_{o}\right)^{2}+\alpha_{L}} \\
& \otimes \frac{1}{\alpha_{G} \sqrt{\pi}} \exp \left[-\left(\frac{v-v_{o}}{\alpha_{G}}\right)^{2}\right]
\end{aligned}
$$

where, $f_{\mathrm{L}}(\mathrm{v})$ and $f_{\mathrm{G}}(\mathrm{v})$ are the Lorentzian and Gaussian profiles, respectively, $\alpha_{\mathrm{L}}$, and $\alpha_{G}$ are the half-width. The spectral wavelength range is expressed in terms of the initial and final frequency, $v$ and $v_{0}$. The optical transitions near the band edge are resolved at 615.42, 585.43 nm peak absorption wavelengths. Higher energy transitions are identified at 503.55, 481.43, and $433.27 \mathrm{~nm}$. The results are shown in Figure 5-2. 


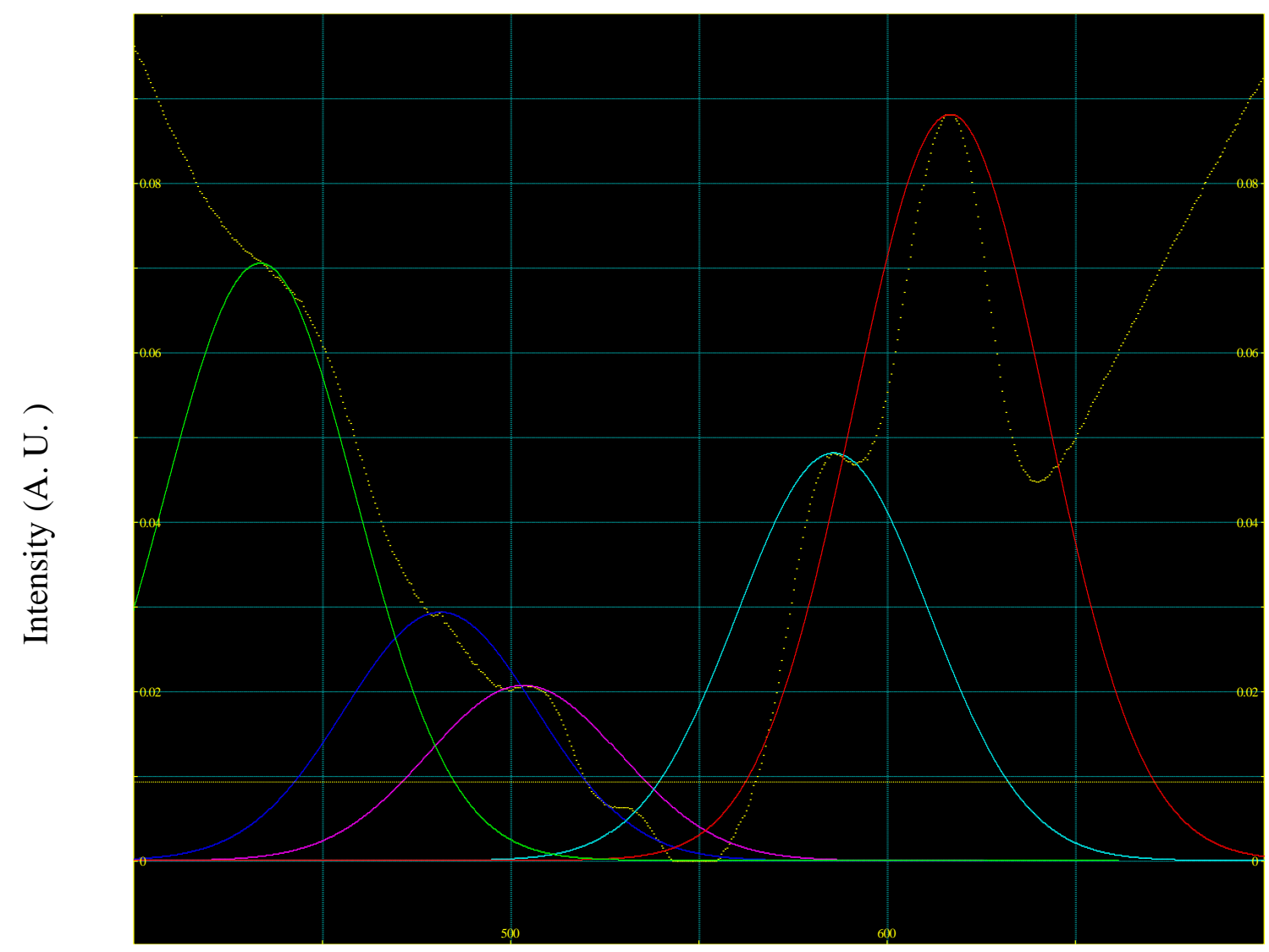

Wavelength (nm)

Figure 5-2: Voigt fit results for CdSe/ZnS:PMMA absorption spectrum at $\mathrm{T}=295 \mathrm{~K}$. The dashed yellow line represents the recorded absorption spectrum and the solid colored lines represent the fit. 
The CdSe/ZnS:PMMA quantum dot nanocomposite was heated from room temperature up to $325 \mathrm{~K}$ and absorption spectra were recorded at 10 degree increments. Figure 5-3 shows the absorption spectra of the CdSe/ZnS QD within the temperature range 295-325 K. The figure inset magnifies the decrease in the first absorption peak. The absorption spectra are observed to red shift with increasing temperature. Plotted in Figure 5-4, the $1 \mathrm{~S}_{\mathrm{e}} \rightarrow 1 \mathrm{~S}_{3 / 2}$ first absorption peak wavelength red shifts by $2 \mathrm{~nm}$ over the temperature range. A quantitative analysis of the temperature dependent change in the $1 S_{3 / 2} \rightarrow 1 S_{e}$ optical transition is complicated because this low energy transition may contain temperature-dependent contributions from higher energy transitions. As shown in Figure 5-5, the contributions show up as a slight broadening in the full width at half maximum (FWHM). Analysis of the $1 S_{e} \rightarrow 2 S_{3 / 2}$ second absorption peak is carried out. Unlike most CdSe/ZnS QD nanocomposite thin films, the CdSe/ZnS:PMMA QD nanocomposite film absorption spectrum clearly shows the first two optical transitions. The peak absorption ratio $\left(1 \mathrm{~S}_{\mathrm{e}} \rightarrow 1 \mathrm{~S}_{3 / 2} \quad / 1 \mathrm{~S}_{\mathrm{e}} \rightarrow 2 \mathrm{~S}_{3 / 2}\right)$ is used as a self-referenced temperature measurement technique for the CdSe/ZnS:PMMA QDs. The temperature dependence of the ratio, $1 \mathrm{~S}_{\mathrm{e}} \rightarrow 1 \mathrm{~S}_{3 / 2} / 1 \mathrm{~S}_{\mathrm{e}} \rightarrow 2 \mathrm{~S}_{3 / 2}$ varies linearly with a 0.98 correlation factor as shown in Figure 5-6. 


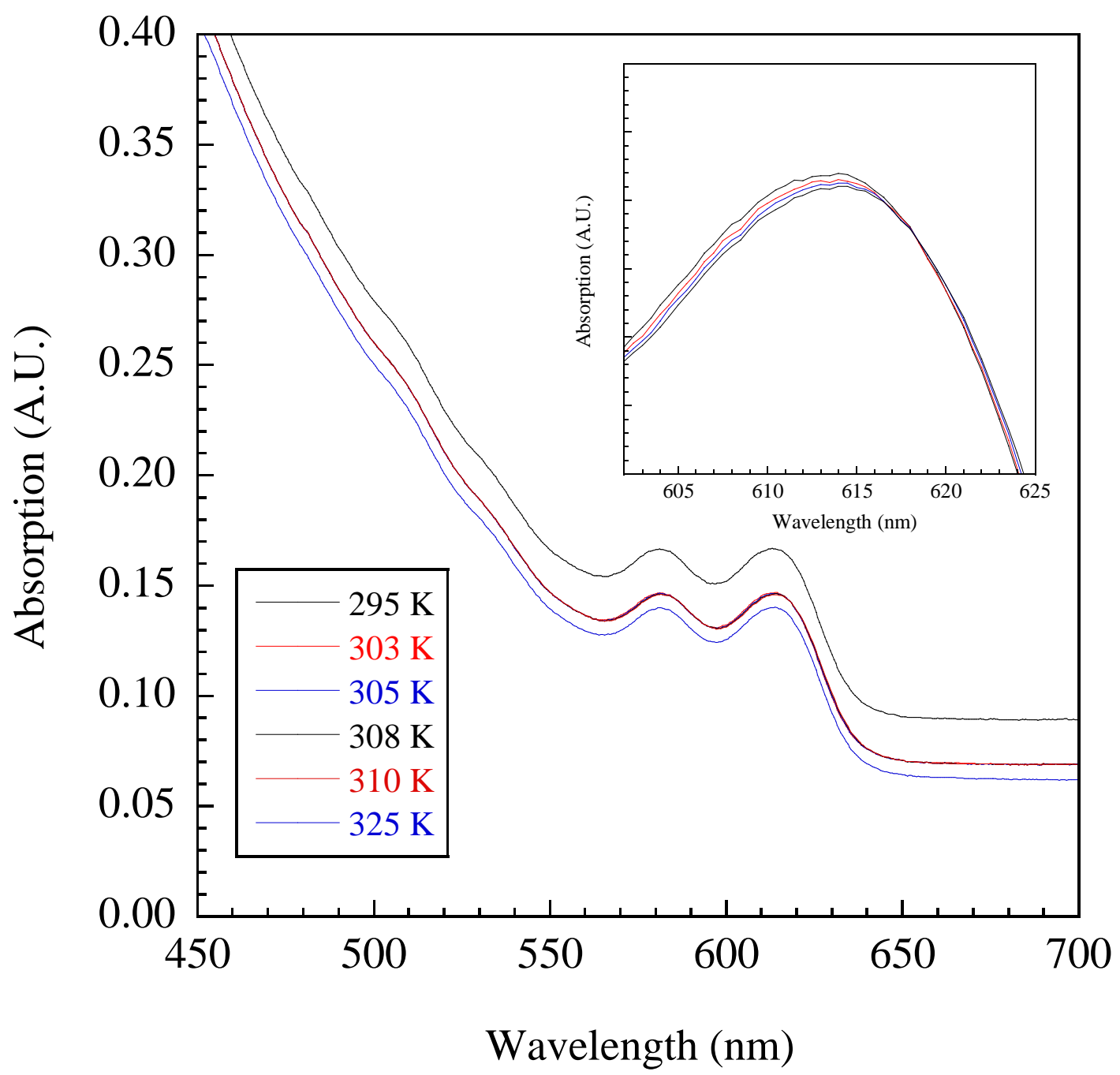

Figure 5-3: Absorption spectra of the CdSe/ZnS:PMMA quantum dot at different temperatures. 


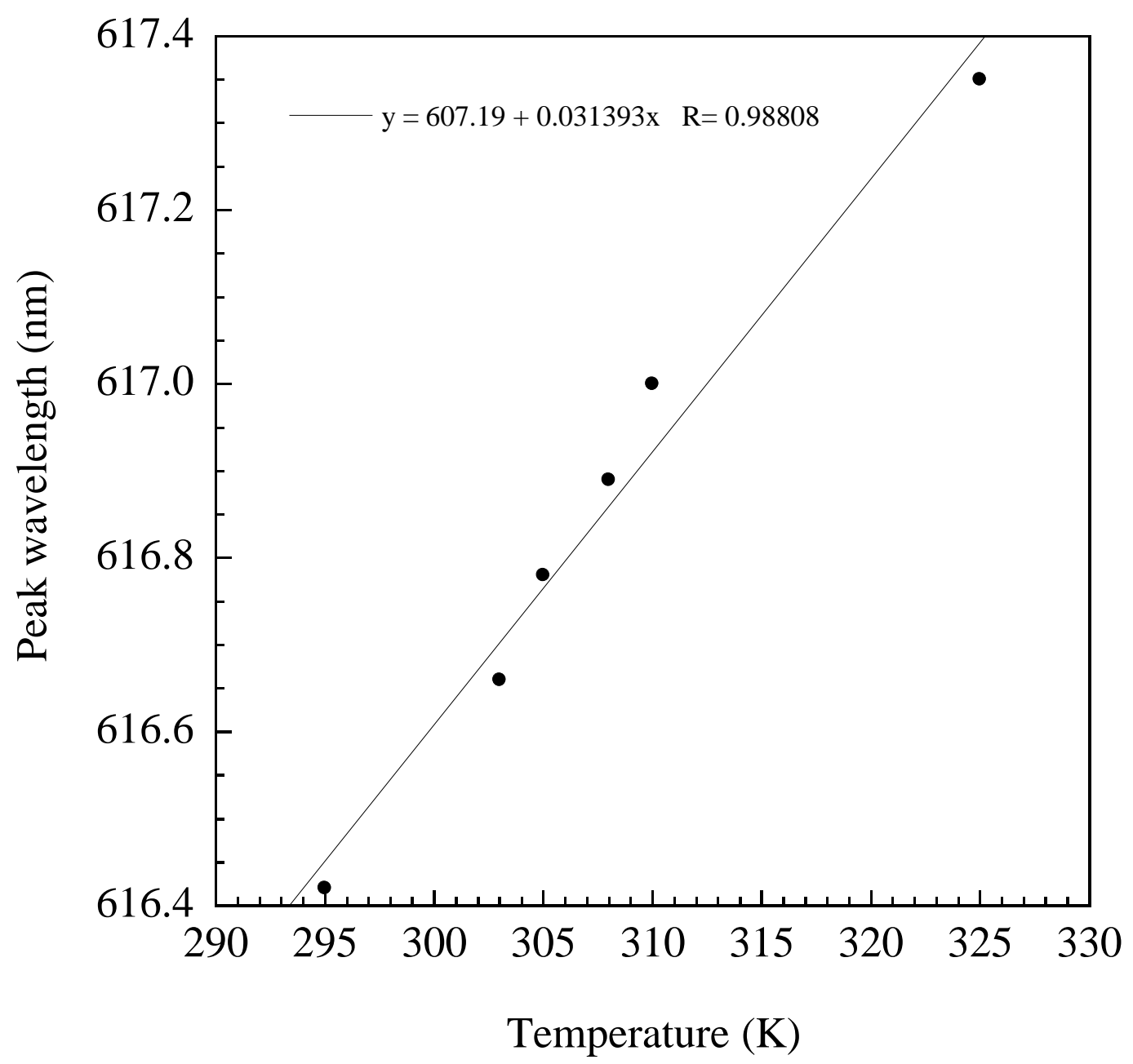

Figure 5-4: Variation in the first exciton absorption peak wavelength with temperature for $\mathrm{CdSe} / \mathrm{ZnS}$ quantum dots in PMMA. 


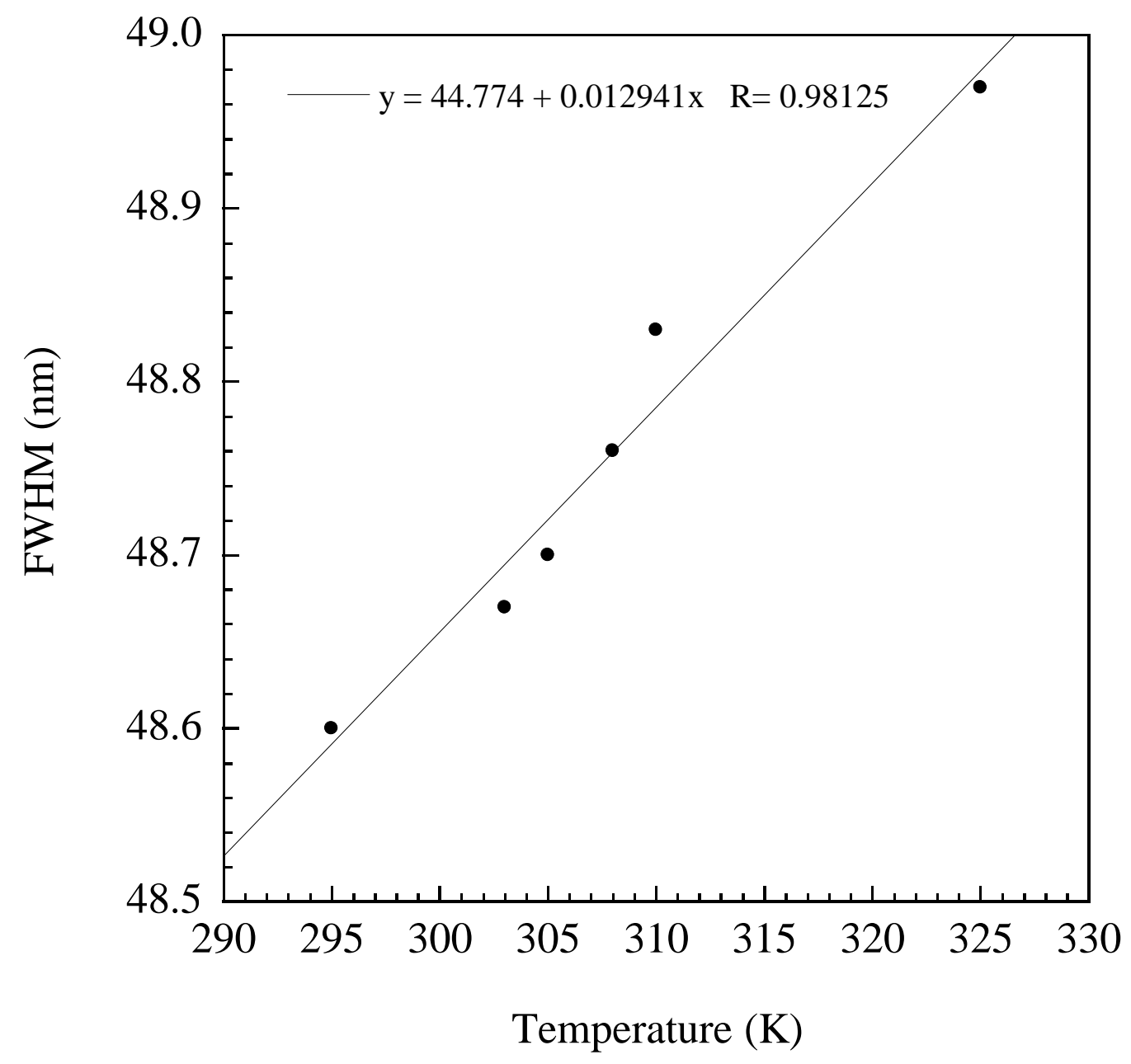

Figure 5-5: Variation in the full width at half maximum (FWHM) with temperature for $\mathrm{CdSe} / \mathrm{ZnS}$ quantum dots in PMMA. 


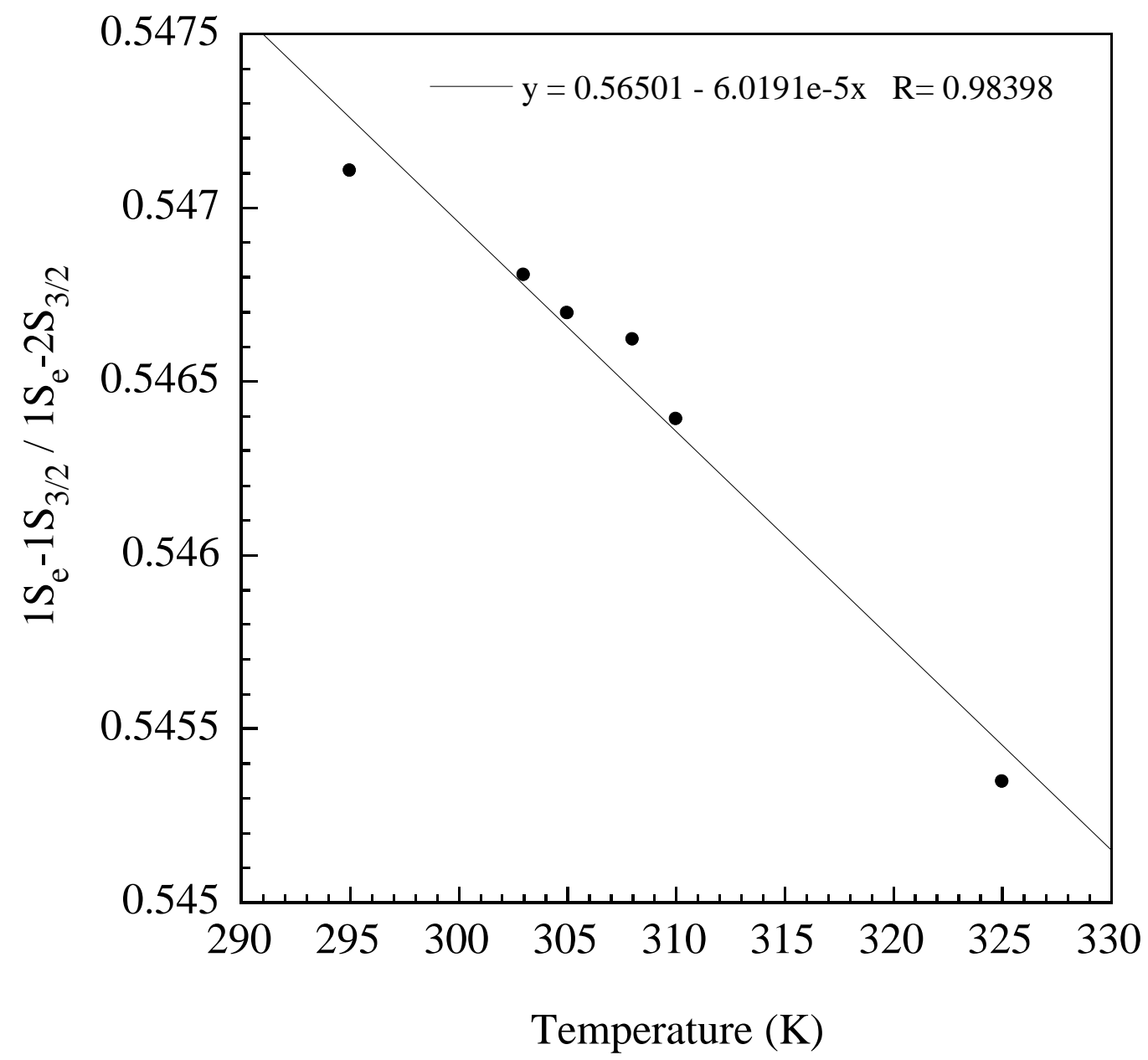

Figure 5-6: The peak absorption ratio, $1 \mathrm{~S}_{\mathrm{e}} \rightarrow 1 \mathrm{~S}_{3 / 2} / 1 \mathrm{~S}_{\mathrm{e}} \rightarrow 2 \mathrm{~S}_{3 / 2}$ versus temperature. 
Temperature dependent absorption cross section calculations were carried out from first principles. The quantum dot radius is calculated from the effective mass approximation model adapted from Equation 2.6:

$$
E=E_{g(b u l k)}+\frac{\hbar^{2} \pi^{2}}{2 \mu R^{2}}
$$

where, $\mathrm{E}$ is the quantum dot energy band gap, $\mathrm{E}_{\mathrm{g}(b u l k)}$ is the band gap of bulk CdSe, $\hbar$ is the reduced Planck's constant, $\mathrm{R}$ is the particle size, and $\mu$ denotes the effective mass. The parameters used for the CdSe/ZnS:PMMA quantum dot nanocomposite are $\mathrm{E}_{\mathrm{g}(b u l k)}=1.74 \mathrm{eV}, \hbar=1.05 \times 10^{-34} \mathrm{Js}$, and $\mu=9.187 \times 10^{-32} \mathrm{~kg}$. A $4.02 \mathrm{~nm}$ particle size is calculated from the $628 \mathrm{~nm}(\mathrm{E}=1.97 \mathrm{eV})$ absorption onset.

The concentration of quantum dot absorption sites $(\mathrm{N})$ is the total sample mass of the QD nanocomposite divided by the mass of a one quantum dot. The mass of the CdSe/ZnS:PMMA QD film is measured to $0.23 \mathrm{~g}$. For spherical quantum dots, the volume of one quantum dot $\left(\mathrm{V}_{\mathrm{QD}}\right)$ is given by $\mathrm{V}_{\mathrm{QD}}=4 / 3 \pi \mathrm{r}^{3}$, where $\mathrm{r}$ is the dot size. From Chapter 2, Figure 2.1 the volume of a CdSe unit cell is $V_{c}=a^{2} \operatorname{csin} 60^{\circ}$, where $a=$ $4.29 \AA$ and $\mathrm{c}=7.01 \AA$ are the lattice parameters. The number of units cells in the QD is calculated $\mathrm{V}_{\mathrm{QD}} / \mathrm{V}_{\mathrm{c}}$. The total atoms in the $\mathrm{QD}$ are calculated by multiplying the number of unit cells by 4 , the total atoms in a single wurtzite unit cell. Using the number of CdSe unit cells $\left(\mathrm{N}_{c}\right)$ and the molecular weight of a CdSe molecule $\left(\mathrm{w}_{C d S e}\right)$, the mass $\left(\mathrm{m}_{Q D}\right)$ of a $4.0 \mathrm{~nm}$ sized CdSe quantum dot is approximated as: 


$$
\begin{aligned}
& w_{Q D}=N_{c} w_{C d S e} \\
& m_{Q D}=w_{Q D} / N_{A}
\end{aligned}
$$

where, $\mathrm{N}_{A}$ is Avogadro's number. The mass of a $4.0 \mathrm{~nm}$ sized CdSe quantum dot is calculated to $7.72 \times 10^{-19} \mathrm{~g}$. The concentration of QD absorbers, $\mathrm{N}=3.85 \times 10^{17} \mathrm{~cm}^{-3}$. Beer's Law is used to calculate the absorption cross sections, $\sigma_{a}$. Defined in Chapter 2, Equation 2.12 can be written:

$$
\sigma_{a}=-\frac{1}{N l} \ln (T)
$$

where, $\mathrm{N}$ is the concentration of absorbers, $l$ is the film thickness, and $\mathrm{T}$ is the measured transmission. The optical absorption cross section at room temperature and $325 \mathrm{~K}$ is plotted in Figure 5-7. 


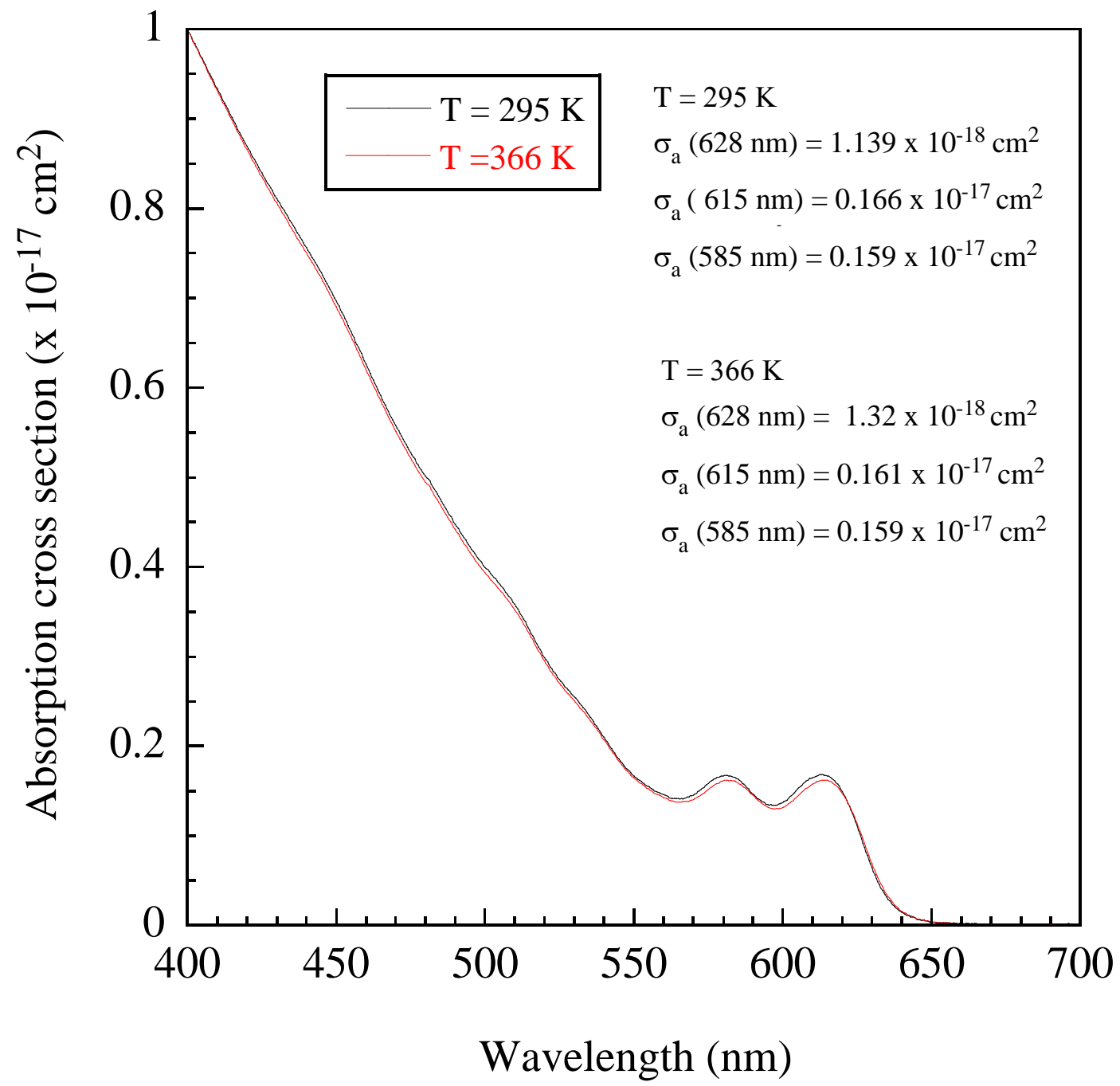

Figure 5-7: Absorption cross section at $\mathrm{T}=295 \mathrm{~K}$ and $\mathrm{T}=325 \mathrm{~K}$ for a $\mathrm{CdSe} / \mathrm{ZnS}: \mathrm{PMMA}$ nanocomposite thin film. 
The results at the absorption onset are fitted to local field approximation theory presented in Chapter 2 where $\sigma_{\mathrm{a}}$ is expanded to

$$
\sigma_{a}=\left(\frac{4}{3} \pi a^{3}\right) \frac{n_{1}}{n_{2}}\left|\frac{3 \varepsilon_{1}}{2 \varepsilon_{1}+\varepsilon_{2}}\right|^{2}\left(2 n_{1} k_{1}\right)
$$

where $a$ is the QD radius, $n_{1}=2.5$ and $n_{2}=1.49$ are the refractive index of the QD and the matrix, $\varepsilon_{1}=10.2$ and $\varepsilon_{2}=3.9$ are the QD and medium relative permittivity [82], and the product $2 \mathrm{n}_{1} k_{1}$ is the bulk CdSe absorption coefficient. At $628 \mathrm{~nm}$, the room temperature experimental QD absorption cross section is $\sigma_{\mathrm{a}}=1.14 \times 10^{-17} \mathrm{~cm}^{2}$. This value is 6 orders of magnitude stronger than CdSe bulk absorption cross section, $\sigma_{\mathrm{a}}=5.57 \times 10^{-23} \mathrm{~cm}^{2}$.

\subsection{Temperature Dependent Photoluminescence Spectroscopy}

Figure 5-7 shows the room temperature measurement of the steady state photoluminescence spectrum of the $\mathrm{CdSe} / \mathrm{ZnS}$ quantum dot nanocomposite in a polymethl-methacrylate (PMMA) matrix. As shown in the figure, the peak PL emission Stokes shifts by $14 \mathrm{~nm}$ with respect to the first QD exciton absorption peak. The dots were excited well above the band edge with a diode pumped solid state (DPSS) laser operating at $532 \mathrm{~nm}$. The experimental test bed used is shown in the previous chapter, Figure 4-6. The band-edge exciton recombination gives rise to a Gaussian emission profile. The temperature dependence of the PL emission is plotted in Figure 5-8. In a typical run, a disk of CdSe/ZnS:PMMA quantum dots were cycled between 295 to $366 \mathrm{~K}$. The dots were irradiated during the entire experiment and the PL spectra were measured 


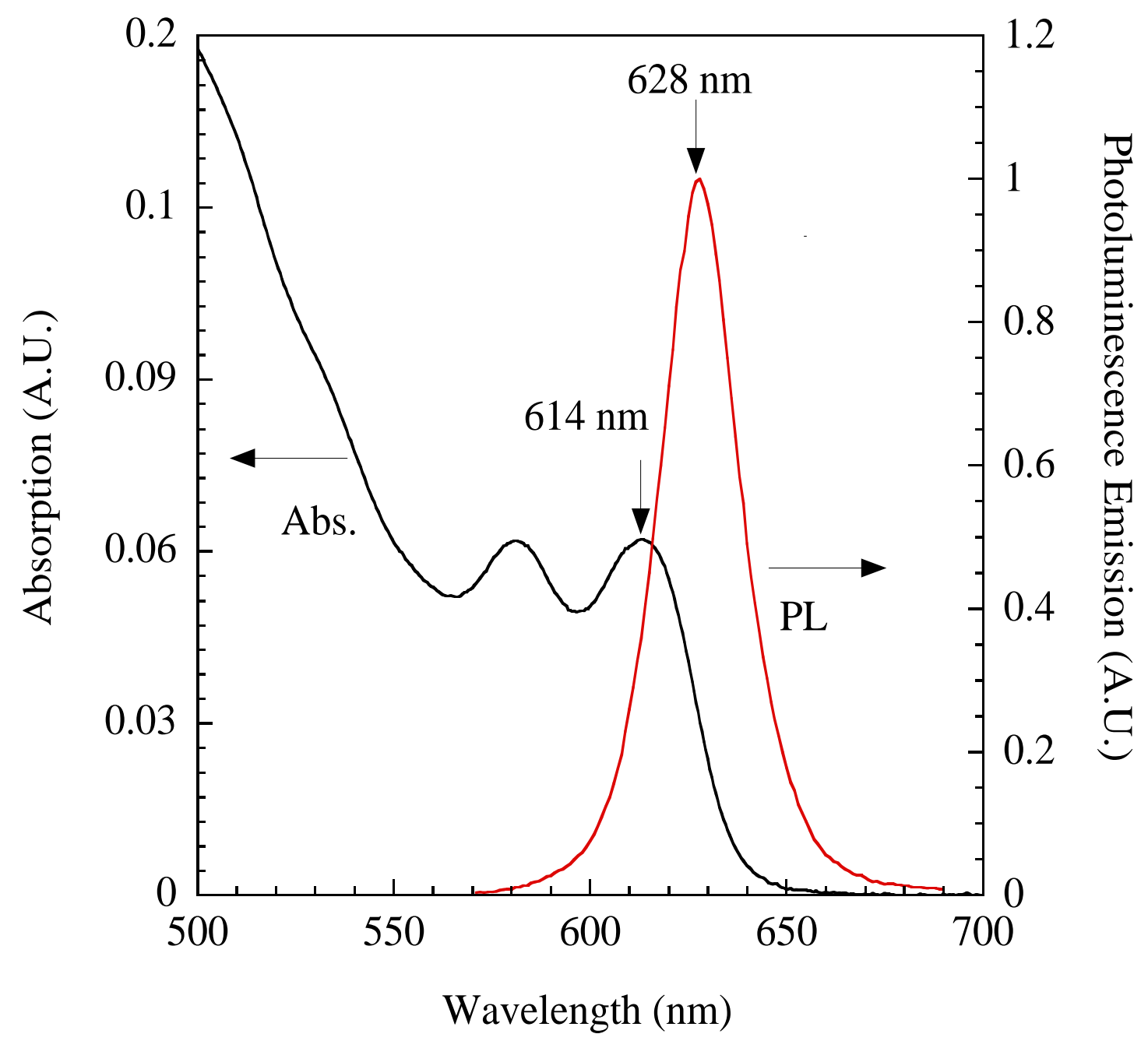

Figure 5-7: Absorption and photoluminescence emission at $295 \mathrm{~K}$ for CdSe/ZnS:PMMA nanocomposite film. 


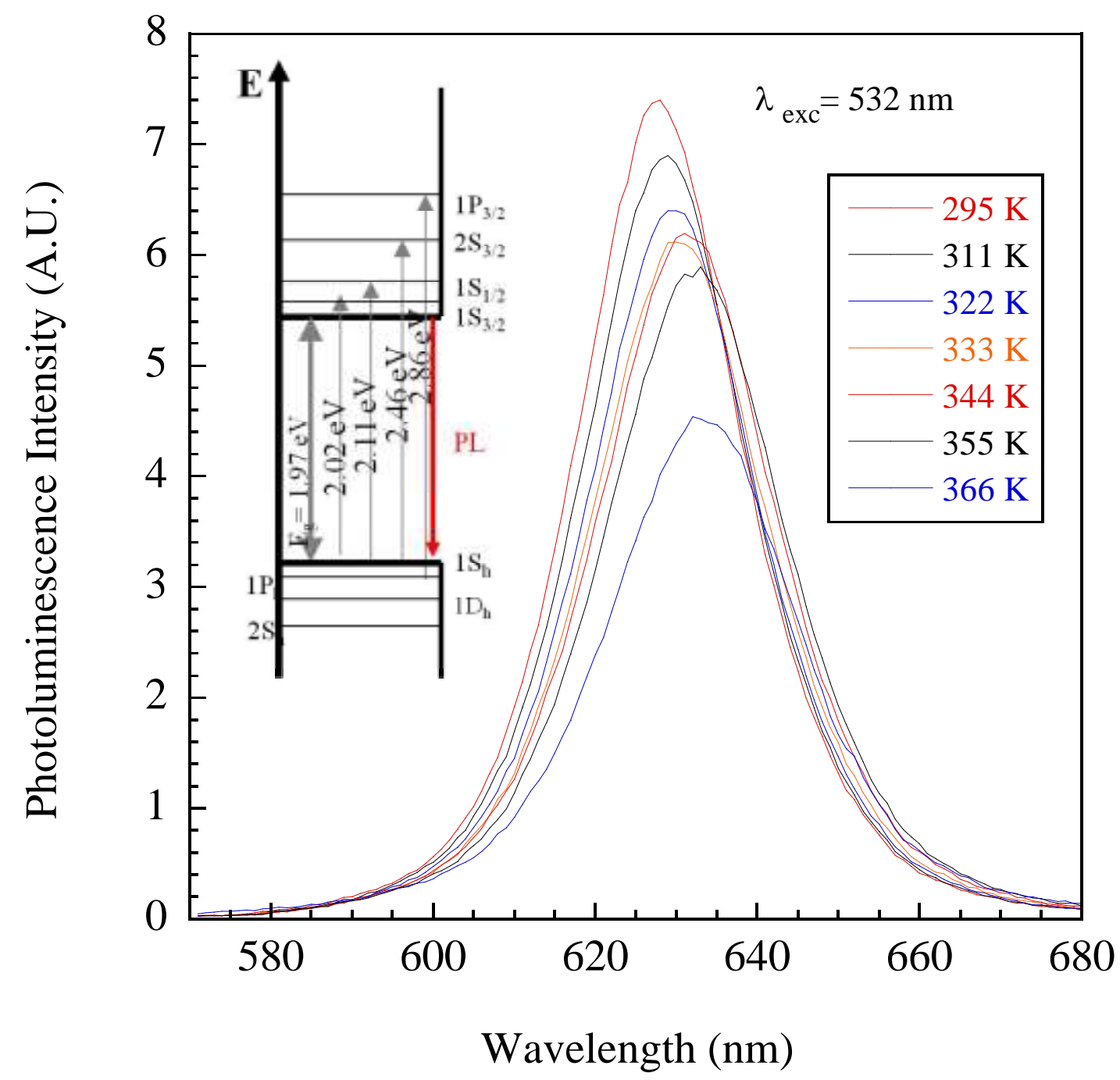

Figure 5-8: $\mathrm{CdSe} / \mathrm{ZnS}$ : PMMA photoluminescence emission measured as a function of temperature. The red arrow in the figure inset represents the CdSe/ZnS QD energy band gap. 


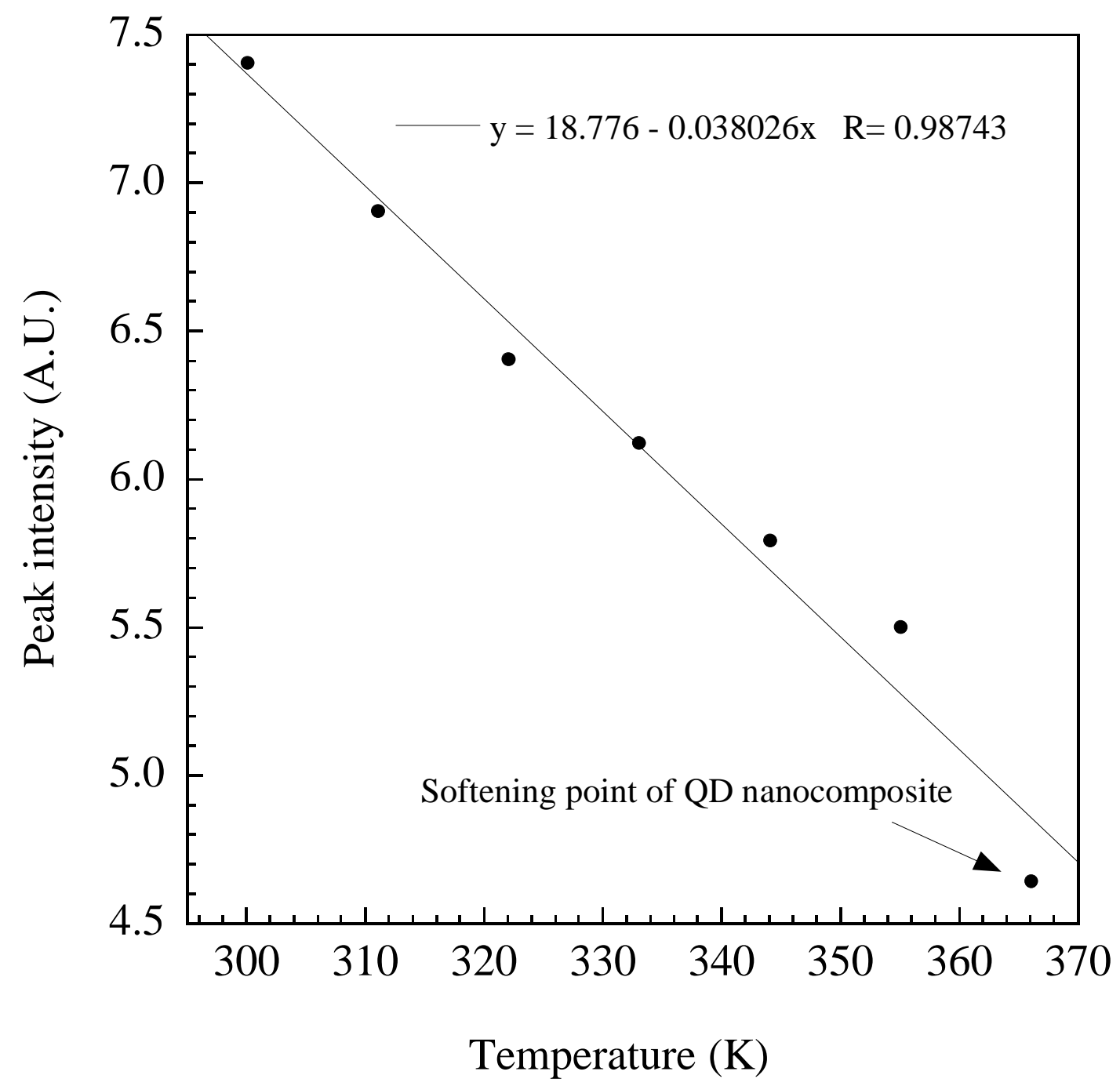

Figure 5-9: Dependence of photoluminescence peak intensity with respect to temperature for CdSe/ZnS:PMMA film. 
at each temperature. The peak PL emission wavelength red shifts by $6 \mathrm{~nm}$ over the temperature range. A linear variation in the peak PL intensity with temperature on the order of $4.1 \% /{ }^{\circ} \mathrm{C}$ is shown in Figure 5-9. Thermal activation of the surface trap sites and multiple phonon-assisted thermal escape of excitons has been used to explain a quick decrease in the PL intensity in quantum dots with increasing temperature [23, 83]. However, for the CdSe/ZnS:PMMA nanocomposite, the PL intensity slowly decreases with increasing temperature. This is due to passivation of nonradiative centers by the $\mathrm{ZnS}$ shell. Hence, the temperature dependent band gap energy offset is due to quantum confinement and is characterized using the Varshni relation. Shown in Figure 5-10, a best fit for the experimental data to theory was found for $\alpha=0.25 \times 10^{-4} \mathrm{eV} / \mathrm{K}$ and $\beta=$ $175 \pm 0.5 \mathrm{~K}$. For bulk CdSe materials $\alpha=3.7 \times 10^{-4} \mathrm{eV} / \mathrm{K}$ and $\beta=150 \pm 10 \mathrm{~K}$ [84]. The QD $\beta$-parameter is comparable to the bulk $\beta$ values. However, the CdSe/ZnS:PMMA quantum dot $\alpha$ parameter is significantly lower than bulk. This implies that CdSe/ZnS:PMMA quantum dot nanocomposite has the advantage of better thermal stability than bulk-semiconductor materials which is a benefit for thermal sensing.

The experimental values of the photoluminescence full width at half maximum (FWHM) were obtained from a Gaussian fit of each spectra. Figure 5-11 clearly shows an increase in the FWHM with increasing temperature. The FWHM broadens by $4 \mathrm{~nm}$. To gain some insight into the carrier-phonon scattering processes involved with the CdSe/ZnS:PMMA nanocomposite system, the experimental FWHM data was fitted to Equation 2.18 (also shown in Figure 5-11). A strong agreement between the experimental data and the best-fit curve is obtained with $\Gamma_{\mathrm{inh}}=9 \mathrm{meV}, \Gamma_{\mathrm{LO}}=50 \mathrm{meV}$, and $\gamma_{\mathrm{AC}}=0.015 \mathrm{meV} / \mathrm{K}$ 


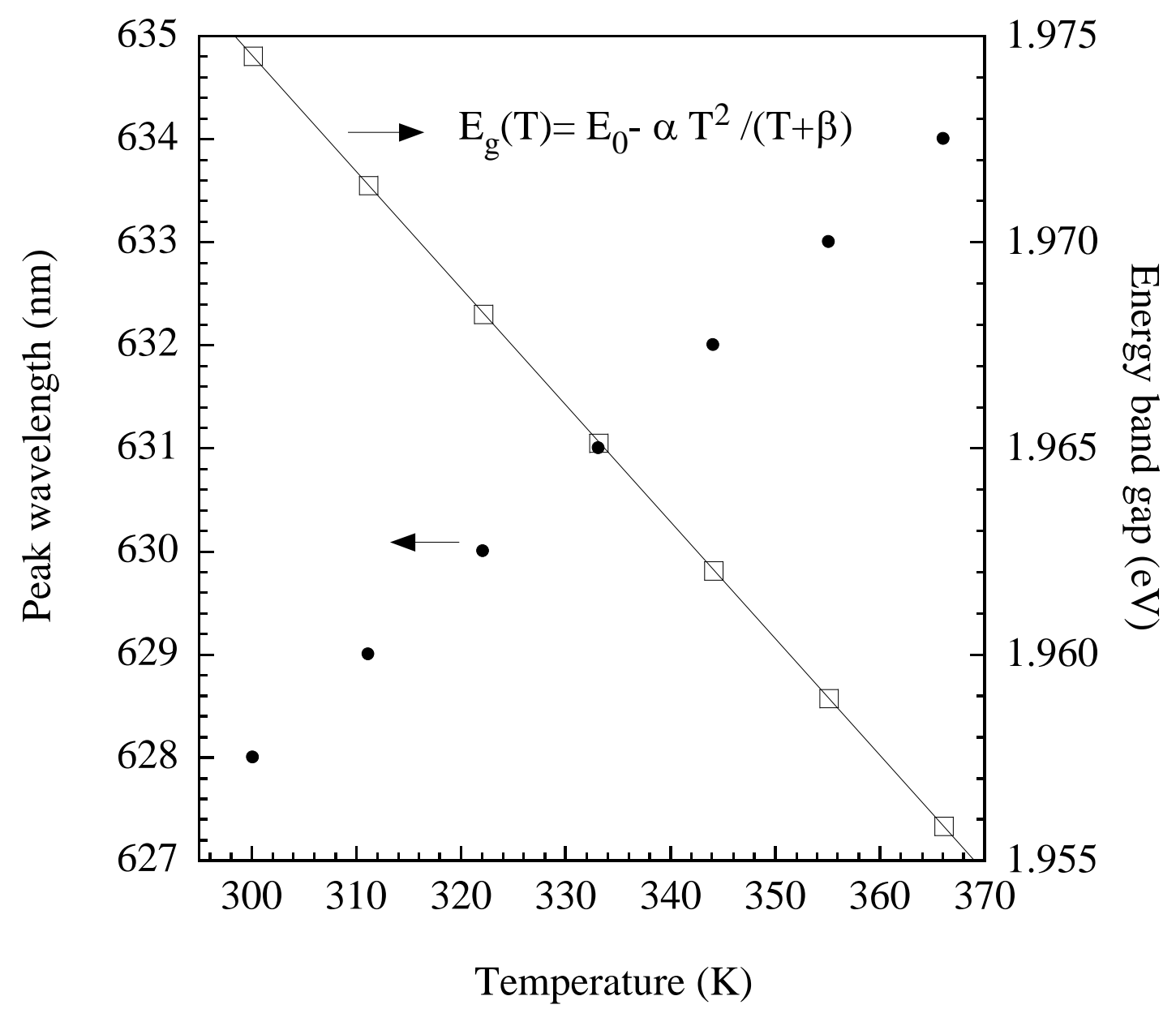

Figure 5-10: PL peak wavelength (circles) versus temperature. Experimental energy band gap for the QD sample (squares) and best fit curve (line). 


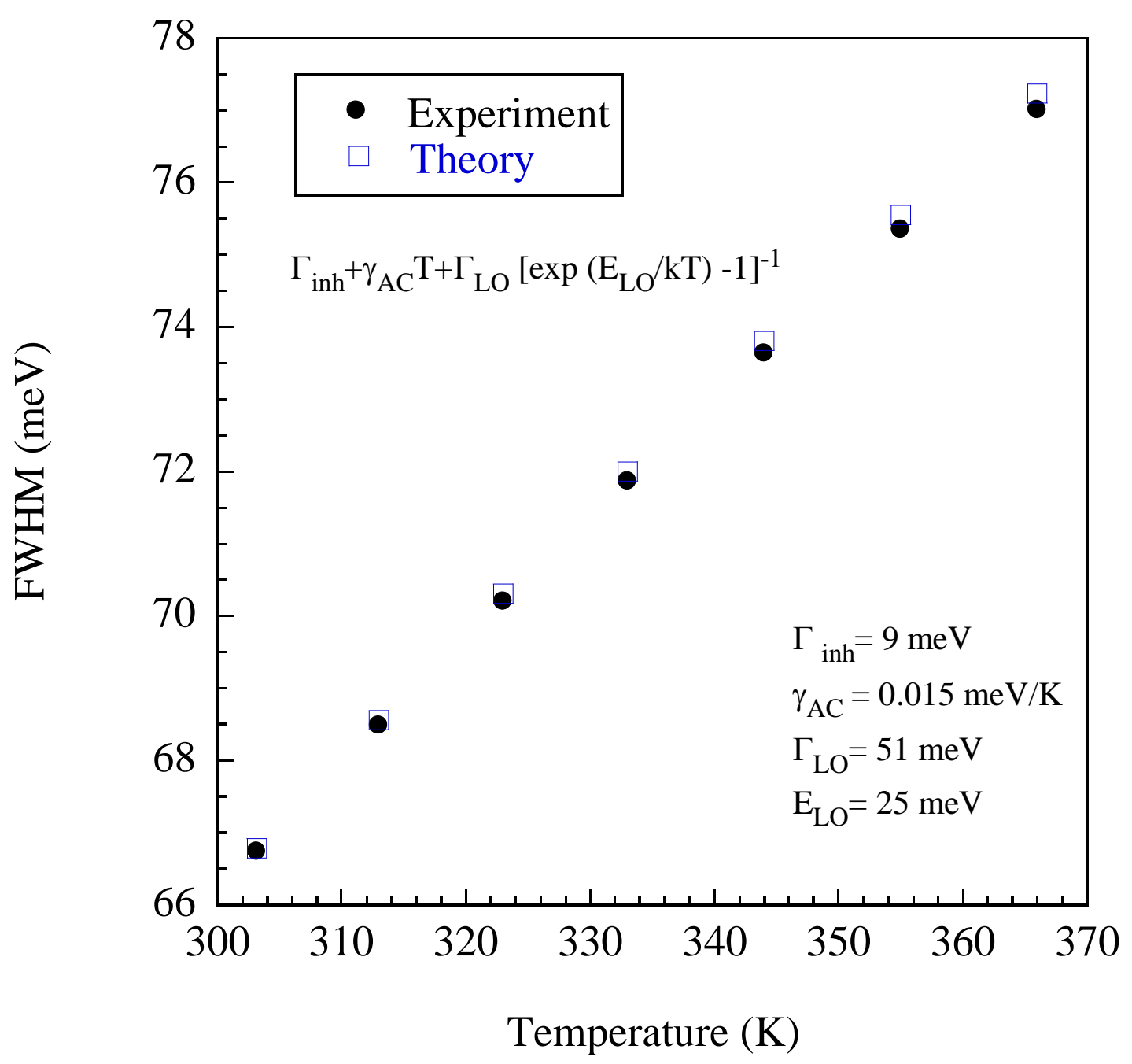

Figure 5-11: Dependence of the CdSe/ZnS:PMMA full width at half maximum (FWHM) with respect to temperature. 
The CdSe/ZnS:PMMA nanocomposite was thermally cycled from 300 - $366 \mathrm{~K}$. Thermal cycling yields a reversible change in PL emission peak wavelength. The nanocomposite response is summarized in Table 5-1 and compared against the existing literature.

Table 5.1 CdSe(ZnS):PMMA Thermal Sensor Response

\begin{tabular}{|c|c|c|c|}
\hline Parameter & This research & Walker [24] & Yu [28] \\
\hline $\mathbf{T}_{\max }$ & $100^{\circ} \mathrm{C}$ & $40^{\circ} \mathrm{C}$ & $90^{\circ} \mathrm{C}$ \\
\hline $\begin{array}{c}\text { Studied } \\
\text { Temperature } \\
\text { Range }\end{array}$ & $22^{\circ} \mathrm{C}-100^{\circ} \mathrm{C}$ & $-173-40^{\circ} \mathrm{C}$ & $30-80^{\circ} \mathrm{C}$ \\
\hline$\sigma_{\mathbf{a}}$ & $1.139-1.32 \times 10^{-18} \mathrm{~cm}^{2}$ & ---- & --- \\
\hline$\Delta \lambda /{ }^{\circ} \mathbf{C}$ & $0.15 \mathrm{~nm} /{ }^{\circ} \mathrm{C}$ & $0.10 \mathrm{~nm} /{ }^{\circ} \mathrm{C}$ & $0.08 \mathrm{~nm} /{ }^{\circ} \mathrm{C}$ \\
& $\left(\lambda_{\mathrm{em}}=628 \mathrm{~nm}\right)$ & $\left(\lambda_{\mathrm{em}}=600 \mathrm{~nm}\right)$ & $\left(\lambda_{\mathrm{em}}=519 \mathrm{~nm}\right)$ \\
\hline$\Delta \mathbf{I} /{ }^{\circ} \mathbf{C}$ & $0.21 \mathrm{~A} . \mathrm{U} . /^{\circ} \mathrm{C}$ & $0.03 \mathrm{~A} . \mathrm{U} . /^{\circ} \mathrm{C}$ & ---- \\
\hline $\mathbf{F W H M} /{ }^{\mathbf{O}} \mathbf{C}$ & $0.03 \mathrm{~nm} /{ }^{\circ} \mathrm{C}$ & $0.28 \mathrm{~nm} /{ }^{\circ} \mathrm{C}$ & ---- \\
\hline $\mathbf{Q . Y}$. & $0.32[85]$ & 0.26 & --- \\
\hline$\tau$ & $30-15 \mathrm{~ns}$ & ---- & ---- \\
\hline
\end{tabular}


Walker et al. [24] measured steady-state PL spectrum of $5.0 \mathrm{~nm} \mathrm{CdSe/ZnS}$ quantum dots dispersed in a PMMA matrix under $480 \mathrm{~nm}$ irradiation. Band-edge recombination of the exciton pairs within the $\mathrm{CdSe}$ core gives rise to near-Gaussian emission profiles. The room temperature peak emission wavelength is $600 \mathrm{~nm}$ at $250 \mathrm{~K}$. Temperature dependent data were recorded in both directions (high to low and low to high) from $278-315 \mathrm{~K}$ and yield a $0.10 \mathrm{~nm} /{ }^{\circ} \mathrm{C}$ sensitivity. In this work, a sensitivity of $0.15 \mathrm{~nm} /{ }^{\circ} \mathrm{C}$ is obtained for extension to $366 \mathrm{~K}$. In 2010 , Yu et al. reported the first investigation on the effect of the PMMA matrix when loaded with quantum dots and subjected to heat treatment [28]. When QDs are subject to temperature changes, the major contributions to changes in the emission are the changes in the band gap energy, refractive index of the surrounding medium, and the quantum dots. For the QD-PMMA system, the latter two components tend not to be as discernible and may cancel each other out due to similarity in the thermo-optic coefficients. The thermo-optic coefficient of $\mathrm{CdSe}$ is of the same magnitude as PMMA $\left(\sim 1.0 \pm 0.3 \times 10^{-4} /{ }^{\circ} \mathrm{C}\right)$ at the emission wavelength [86]. Hence, a matrix material with a larger thermo-optic coefficient and resistance to elevated temperature was sought after this study. For example, sol gel poly methyl methacrylate and modified silicone dioxide, $\mathrm{SiO}_{2}$, with reported coefficients of $2.3 \times 10^{-4} /{ }^{\circ} \mathrm{C}$ and $3.1 \times 10^{-4} /{ }^{\circ} \mathrm{C}$ [86] respectively, can afford the potential for larger wavelength shifts for a given temperature change, and hence a greater overall increased temperature sensor sensitivity. 


\subsection{Temperature Dependent Lifetime Analysis}

The CdSe/ZnS:PMMA absorption cross sections, $\sigma_{\mathrm{a}}$ were calculated in Section 5.1 The oscillator strength was calculated by integrating $\sigma_{\mathrm{a}}$ over the $1 \mathrm{~S}_{3 / 2} \rightarrow 1 \mathrm{~S}_{\mathrm{e}}$ exciton transition absorption band. From small particle theory, the oscillator strength $f$ is given by [87]

$$
f=\frac{4 m \varepsilon_{o} c}{e^{2}} \int_{v_{1}}^{v_{2}} \sigma_{a}(v) d v
$$

where, $v_{1}$ and $v_{2}$ are the initial and end frequencies of the first exciton absorption band, $m$ is the mass of an electron, $c$ is the speed of light, $\varepsilon_{\mathrm{o}}$ is the permittivity of free space, and e denotes the charge of an electron. The radiative lifetime, $\tau$ is extracted using

$$
\tau=\left[\frac{8 \pi}{\lambda^{2}} \int_{v_{1}}^{v_{2}} \sigma_{a}(v) d v\right]^{-1}
$$

where $\lambda$ is the wavelength of the $1 S_{3 / 2} \rightarrow 1 S_{e}$ first transition peak. For $v_{1}=5.00 \times 10^{14} \mathrm{~Hz}$ and $v_{2}=4.76 \times 10^{14} \mathrm{~Hz}$, the oscillator strength and radiative lifetime were calculated and are reported in Table 5-2, columns 5 and 7.

Table 5.2 Oscillator strength and radiative lifetime of $1 S_{3 / 2} \rightarrow 1 S_{e}$ first absorption peak.

\begin{tabular}{|c|c|c||c|c||c|c|}
\hline $\begin{array}{c}\text { Temperature } \\
(\mathrm{K})\end{array}$ & $\begin{array}{c}1 \mathrm{~S}_{3 / 2} \rightarrow 1 \mathrm{~S}_{\mathrm{e}} \\
\text { band } \\
(\mathrm{nm})\end{array}$ & $\begin{array}{c}1 \mathrm{~S}_{3 / 2} \rightarrow 1 \mathrm{~S}_{\mathrm{e}} \\
\text { peak } \\
(\mathrm{nm})\end{array}$ & $\begin{array}{c}\text { PL } \\
\text { emission } \\
(\mathrm{nm})\end{array}$ & $\begin{array}{c}\text { Oscillator } \\
\text { Strength } \\
\text { Experiment }\end{array}$ & $\begin{array}{c}\text { Oscillator } \\
\text { Strength } \\
\text { Theory }\end{array}$ & $\begin{array}{c}\text { Radiative } \\
\text { Lifetime } \\
(\mathrm{ns})\end{array}$ \\
\hline \hline 295 & $598-628$ & 615.5 & 628 & 1.70 & 1.67 & 29.5 \\
\hline 366 & $598-628$ & 617 & 634 & 1.56 & 1.68 & 15.0 \\
\hline
\end{tabular}

${ }^{*}$ Equation 5.8 
In strong quantum confinement, the oscillator strength, $\mathrm{f}_{\mathrm{osc}}$ is

$$
f_{\text {osc }}=\frac{4}{\sqrt{3}} \pi \frac{a_{b}^{3} \omega_{b u l k}}{a^{2} c \omega_{Q F D}} f_{b u l k}
$$

where $a_{\mathrm{b}}$ is the exciton Bohr radius, $\omega_{\mathrm{bulk}}$ is the bulk emission frequency, $\omega_{\mathrm{QD}}$ is the quantum dot emission frequency, $\mathrm{f}_{\text {bulk }}$ represents the oscillator strength in bulk CdSe, and $\mathrm{a}$ and $\mathrm{c}$ are the w-CdSe lattice constants. For CdSe/ZnS:PMMA QD, $a_{\mathrm{b}}=4.0 \mathrm{~nm}$, $\omega_{\mathrm{QD}}=4.5 \times 10^{15} \mathrm{rad} / \mathrm{s}, \quad \mathrm{a}=0.429 \mathrm{~nm}$, and $\mathrm{c}=0.7015 \mathrm{~nm} . \quad$ For bulk CdSe, $\omega_{\text {bulk }}=2.99 \times 10^{15} \mathrm{rad} / \mathrm{s}$ and $\mathrm{f}_{\text {bulk }}=5 \times 10^{-4}$ per CdSe unit cell [88]. The $\mathrm{f}_{\text {osc }}=1.67$ at $\mathrm{T}=295 \mathrm{~K}$ is in strong agreement with experiment. 


\title{
Chapter 6 \\ THERMOMETRY USING QUANTUM DOT \\ $\mathrm{CdSe} / \mathrm{ZnS}: \mathrm{SiO}_{2}$ NANOCOMPOSITE
}

\begin{abstract}
“He leads us. He doesn't push us. He doesn't hand us a map and send us on our way without Him. He wants us to keep our eyes on Him, and follow him one step at a time."
\end{abstract}

Joyce Meyer

\subsection{Temperature Dependent Absorption Spectroscopy}

The optical absorption spectra of the $\mathrm{CdSe} / \mathrm{ZnS}: \mathrm{SiO}_{2}$ quantum dot solutions and thin films were recorded using a Perkin Elmer Lambda 900 spectrophotometer in the spectral range 400 - $700 \mathrm{~nm}$. Experimental details are provided in Chapter 4. The concentration of QDs in methanol was $2.5 \mathrm{mg} / \mathrm{mL}$. Four vials of CdSe/ZnS quantum dots with $520 \mathrm{~nm}, 560 \mathrm{~nm}, 609 \mathrm{~nm}$, and $680 \mathrm{~nm}$ photoluminescence peak wavelengths were purchased from N.O.M. Inc. (see Chapter 5 for experimental details). The samples are identified as Sample A, B, C, and D respectively. The optical absorption spectra on the as-received $\mathrm{CdSe} / \mathrm{ZnS}: \mathrm{CH}_{3} \mathrm{OH}$ QD nanocomposite solutions are shown in Figure 6-1. The band structure was analyzed using PeakFit v4.12 graphical analysis software [89]. Sample A shows a first absorption peak at $496.2 \mathrm{~nm}$. The first exciton peak is clearly observed in Sample B at $532.54 \mathrm{~nm}$. However, samples A, B, and D do not show the fine structure characteristic of quantum confinement in CdSe semiconductor quantum dots [90-92]. Sample D has an absorption onset at $680 \mathrm{~nm}$ which approaches the bulk CdSe energy band gap. The structureless 


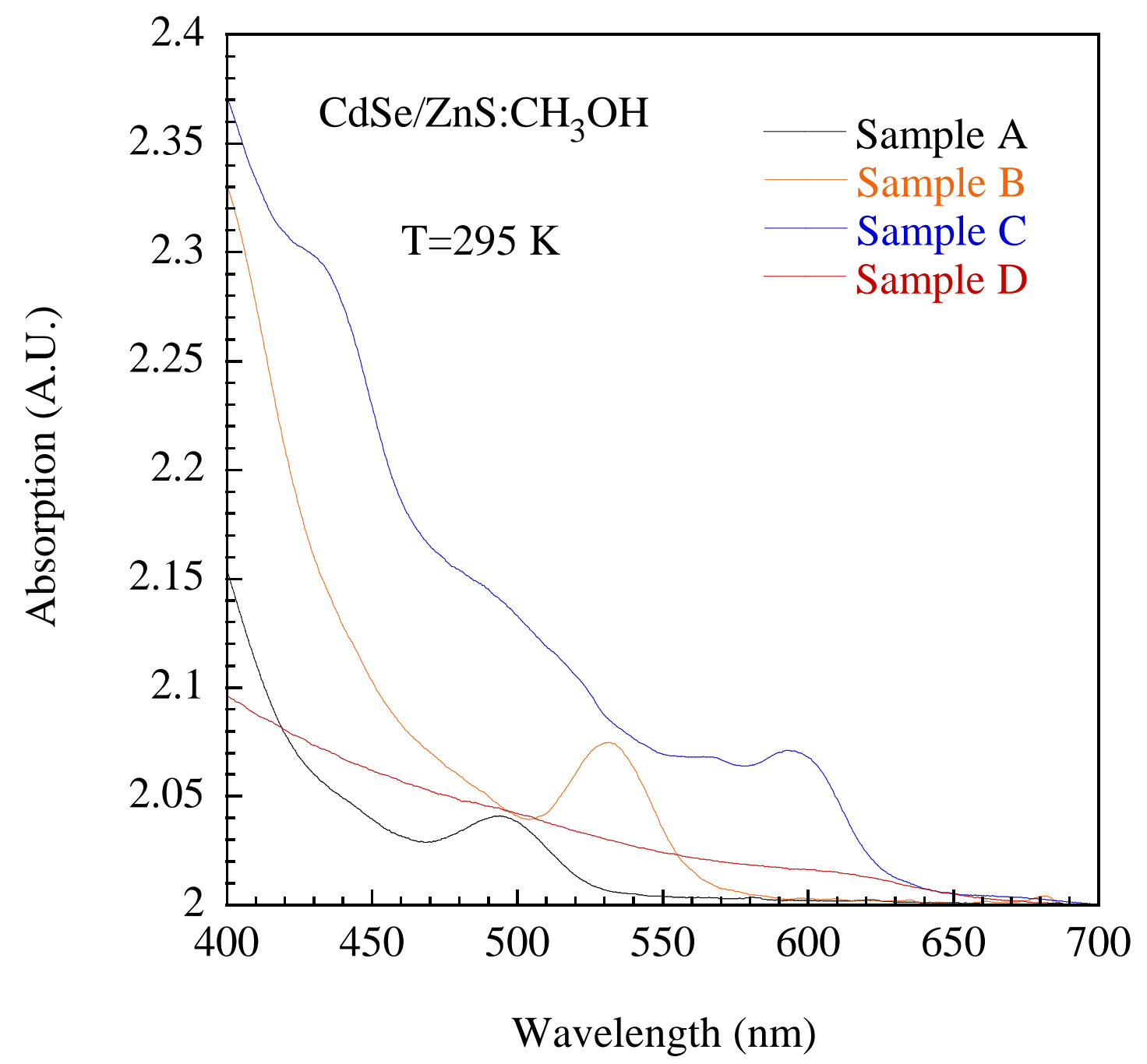

Figure 6-1: Room temperature $\mathrm{CdSe} / \mathrm{ZnS}: \mathrm{CH}_{3} \mathrm{OH}$ nanocomposite solution absorption spectra for Sample A, Sample B, Sample C, and Sample D. 
absorption profile of Sample D indicates the electrons penetrate into the conduction band continuum such that the quantum size effect is not observed. The discrete nature of QD states is often concealed due to inhomegeneities such as variation in dot shape, size distributions, and stoichiometry. The $\mathrm{CdSe} / \mathrm{ZnS}: \mathrm{CH}_{3} \mathrm{OH}$ QDs had a high degree of monodispersity $(<2 \%$ per manufacture specifications). Stoichiometric data was proprietary per the manufacturer. The quantum size effect clearly shows in Sample C with three absorption bands at $1 \mathrm{~S}_{3 / 2} \rightarrow 1 \mathrm{~S}_{\mathrm{e}}, 2 \mathrm{~S}_{3 / 2} \rightarrow 1 \mathrm{~S}_{\mathrm{e}}$, and $1 \mathrm{P}_{3 / 2} \rightarrow 1 \mathrm{P}_{\mathrm{e}}$. Shown in Figure 6-2, the first three quantum dot resonances of the $50 \mu \mathrm{m}$ thick $\mathrm{CdSe} / \mathrm{ZnS}: \mathrm{SiO}_{2}$ nanocomposite thin film were reproduced by the superposition of three Gaussian bands. The first exciton absorption peak dominates at $2.07 \mathrm{eV}$, while the other two peaks are found in the shoulder at $2.50 \mathrm{eV}$ and at $2.87 \mathrm{eV}$. The $\mathrm{CdSe} / \mathrm{ZnS}: \mathrm{SiO}_{2}$ solution and nanocomposite film optical absorption spectra exhibit a $100 \mathrm{~nm}$ blue shift with respect to the $700 \mathrm{~nm}$ fundamental bulk CdSe absorption onset. This critical observation indicates that the QD nanoparticles are smaller than the Bohr radius of bulk CdSe and confirms that the reported observations are in the strong quantum confinement regime. The bulk CdSe absorption spectrum exhibits an absorption band edge at $711 \mathrm{~nm}[93] . \quad$ As shown in Figure 6-3, a rather uniform featureless absorption spectrum is found in bulk CdSe. In contrast, the absorption spectrum for Sample C QDs appears as a series of overlapping peaks with a well resolved peak near the absorption band edge. 


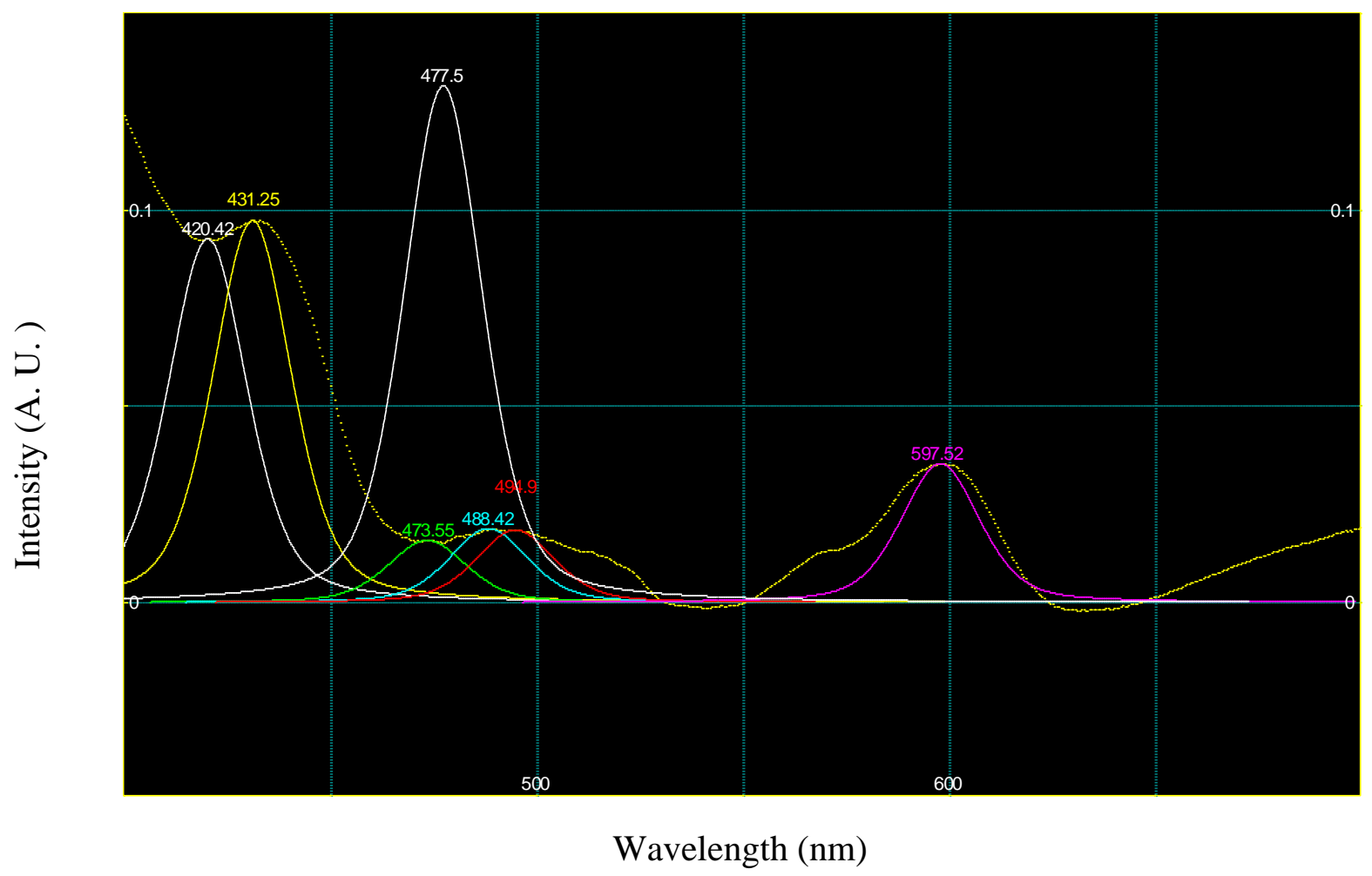

Figure 6-2: Absorption spectrum of $\mathrm{CdSe} / \mathrm{ZnS}: \mathrm{SiO}_{2}$ at $\mathrm{T}=295 \mathrm{~K}$ The yellow dashed line is the absorption spectrum and the solid colored lines represent the fit. 


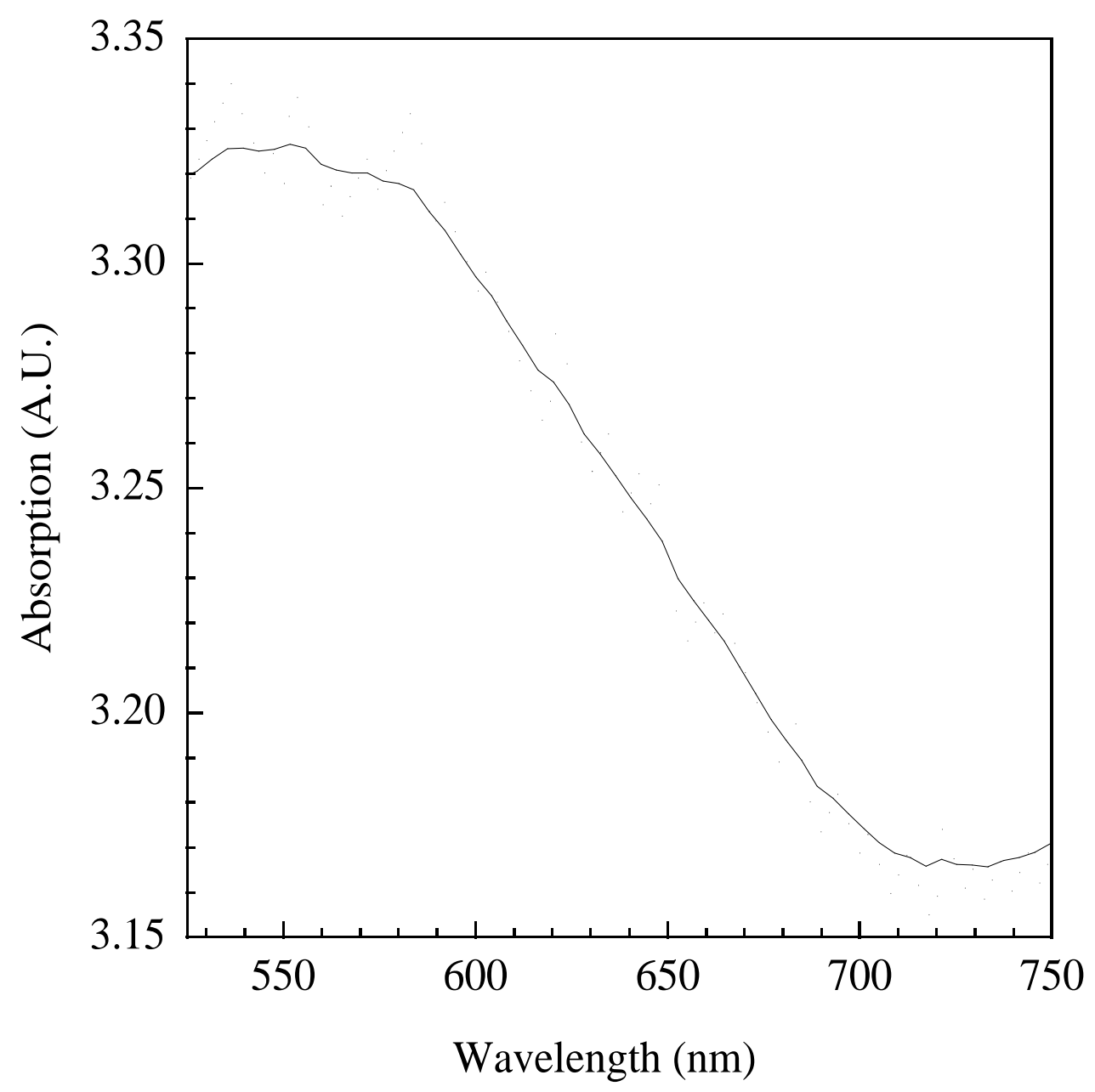

Figure 6-3: Bulk CdSe sample absorption spectra [93]. 
The linear absorption coefficient defines the extent of light absorption through a piece of material. However, the absorption coefficient is not a strong measure of light absorption on the per particle or concentration basis in quantum dot nanostructures. Henceforth, the absorption cross section $\left(\sigma_{a}\right)$ over a range of spectral energies, hu was investigated. The $\mathrm{CdSe} / \mathrm{ZnS}: \mathrm{SiO}_{2}$ QD nanocomposite film was investigated using Sample C. The CdSe/ZnS: $\mathrm{SiO}_{2}$ quantum dot absorption cross section at $295 \mathrm{~K}$ is $0.27 \times 10^{-16} \mathrm{~cm}^{2}$. This measurement on the new $\mathrm{CdSe} / \mathrm{ZnS}: \mathrm{SiO}_{2}$ QD nancomposite is in good agreement with the current literature [94-96]. Absorption cross sections for systems with comparable concentration $(2.5 \mathrm{mg} / \mathrm{mL})$ and QD particle size are reported in Table 6.1 .

Table 6.1 Reported colloidal QD optical absorption cross sections.

\begin{tabular}{|c|c||c|c|}
\hline QD System & QD size (nm) & $\sigma_{\mathbf{a}}\left(\mathbf{c m}^{\mathbf{2}}\right)$ & Reference \\
\hline \hline $\mathrm{CdS} / \mathrm{ZnS} /$ toluene & 3.9 & $6.58 \times 10^{-16}$ & Cheng [94] \\
\hline $\mathrm{CdSe} /$ hexane & 3.4 & $3.7 \times 10^{-15}$ & Leatherdale [95] \\
\hline $\mathrm{CdSe} /$ toluene & 3.0 & $1.80 \times 10^{-16}$ & Donega [96] \\
\hline
\end{tabular}

The structure of the absorbance spectrum is a fingerprint of the quantum dot energy levels. Hence, the absorption cross-section is a critical parameter for quantum emitters. In addition, as shown in Fig. 6-4, $\sigma_{a}$ provides a relationship between the QD concentration and the sample optical density. The absorption cross section determines the per-QD photoexcitation density at a given photon fluence. The utility of obtaining 
reproducible quantum dot absorption cross sections is critical to opto-electronic device design. Despite the recognized usefulness of these values, it has not been straightforward to extract cross sections from chemically synthesized quantum dots. Recently, investigators have reported $\mathrm{CdSe}$ and $\mathrm{CdSe} / \mathrm{ZnS} \mathrm{QD}$ cross sections ranging from $\sigma_{\mathrm{a}} \sim 10^{-14}-10^{-18} \mathrm{~cm}^{2}$ using various optical measurement techniques and approximation models [94-97]. A possible explanation for the range of cross section values for QDs in the literature is the inherent difficulty of accurately counting quantum dot nanocrystals versus ions and molecules in bulk crystals. Unlike molecules or other systems with well defined molecular weights, colloidal quantum dots and other chemically synthesized nanostructures do not possess such exact physical properties. This arises, in part, because wet chemistry fabrication techniques do not yet fully control sample size polydispersities. Even under the most sophisticated synthesis processes, colloidal QDs show dot size distributions between 2 - 15\%. To some extent, polydispersity has been ameliorated by the development of improved chemical syntheses that incorporate a surfactant layer to yield high quality QDs with narrow size distributions. In addition to controlling growth kinetics, increased quantum yield and protection from oxidation are reported for ZnS-capped QDs [57, 98].

The $\mathrm{CdSe} / \mathrm{ZnS}: \mathrm{SiO}_{2}$ QD absorption cross section and absorption coefficient at 295 and $550 \mathrm{~K}$ are plotted in Figure 6-4. At $550 \mathrm{~K}$, the first peak absorption wavelength shifts $8 \mathrm{~nm}$ to a peak wavelength at $605 \mathrm{~nm}$ where, $\sigma_{\mathrm{a}}=0.23 \times 10^{-16} \mathrm{~cm}^{2}$. At elevated temperatures, excitons occupy high-energy states. The linear decrease in the absorption cross section is due to thermally induced intraband absorption. The strength of the interaction of electromagnetic radiation with the electronic energy levels the dots 


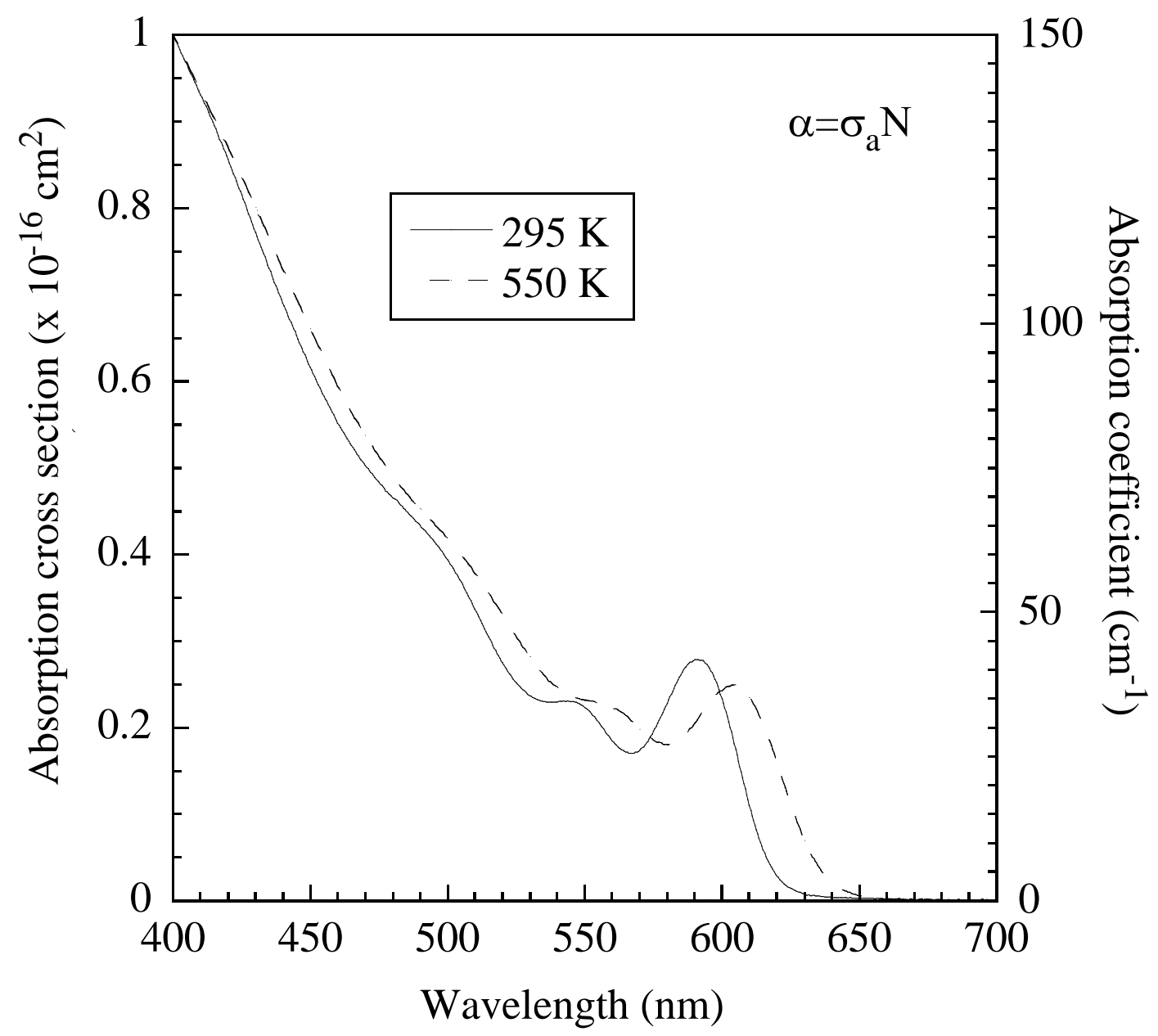

Figure 6-4: Absorption coefficient and absorption cross section for a $50 \mu \mathrm{m}$ $\mathrm{CdSe} / \mathrm{ZnS}: \mathrm{SiO}_{2}$ nanocomposite film. 


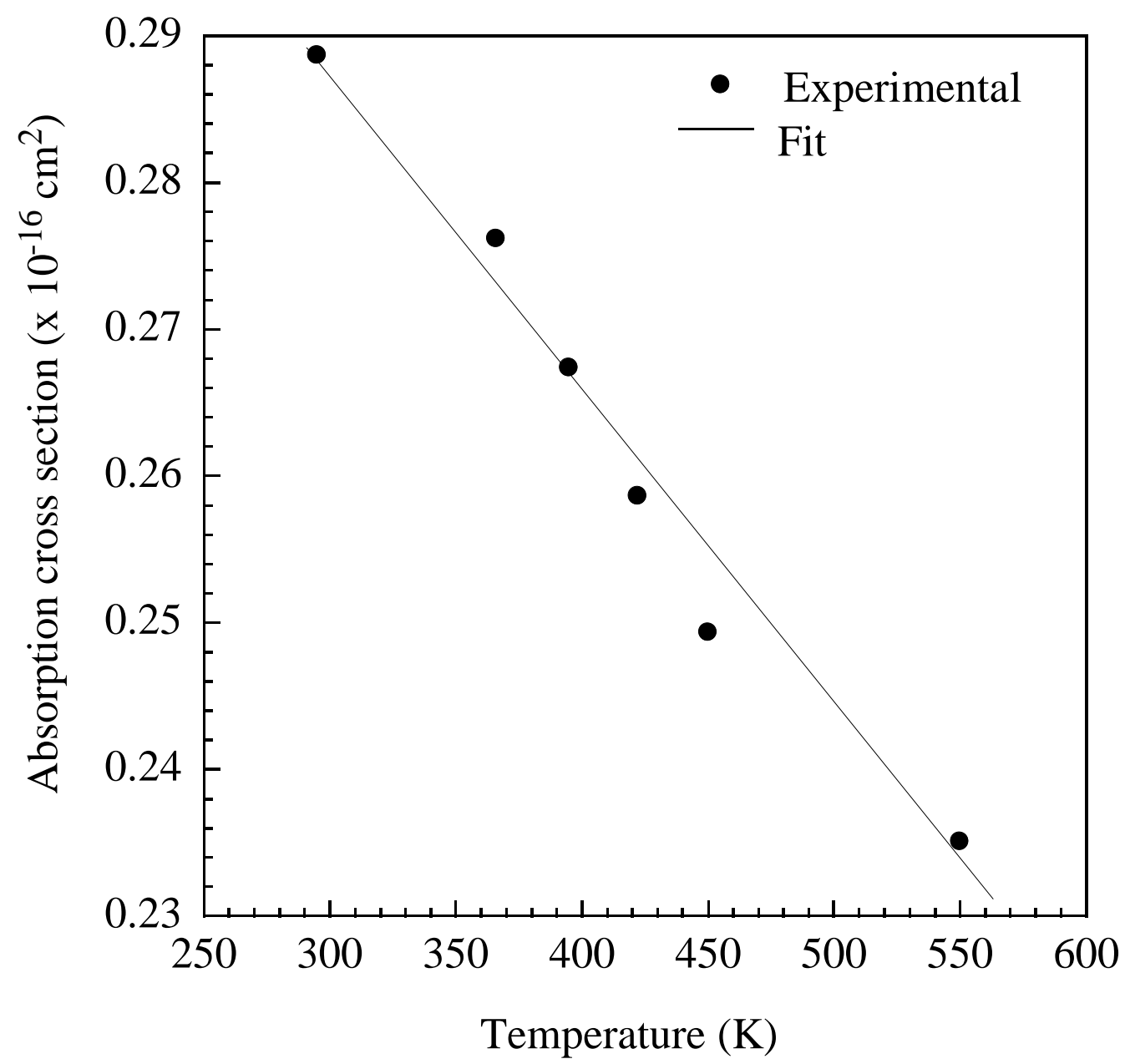

Figure 6-5: Absorption cross section versus temperature at the $1 S_{3 / 2} \rightarrow 1 S_{e}$. 
Table 6.2 Optical absorption cross sections for $\mathrm{CdSe} / \mathrm{ZnS}: \mathrm{SiO}_{2}$ quantum dots at different temperatures.

\begin{tabular}{|c|c|}
\hline Temperature (K) & Absorption cross section $\left(\times 10^{-16} \mathrm{~cm}^{2}\right)$ \\
\hline \hline 295 & 0.283 \\
\hline 366 & 0.271 \\
\hline 395 & 0.260 \\
\hline 422 & 0.251 \\
\hline 450 & 0.243 \\
\hline 550 & 0.232 \\
\hline
\end{tabular}

was investigated by cycling the QD film from 295 to $550 \mathrm{~K}$ and calculating $\sigma_{\mathrm{a}}$. The absorption cross section versus temperature at the absorption onset for the $\mathrm{CdSe} / \mathrm{ZnS}: \mathrm{SiO}_{2}$ quantum dots is plotted in Figure 6-5. The results are summarized in Table 6.2. When compared against bulk crystalline data such as bulk crystalline alexandrite (see Appendix C), the $\mathrm{CdSe} / \mathrm{ZnS}$ QD absorption cross section exceeds the bulk crystalline alexandrite reference sample by a factor of 2 .

The variation in the QD film absorption from the ground state to the first excited state with temperature is plotted in Figure 6-6. Variation in the first absorption peak wavelength with temperature is shown in Figure 6-7. A linear fit yields $\mathrm{R}=0.99$. The change in the full width at half maximum (FWHM) with temperature is shown in Figure 6-8. Non-resonant thermally induced excitation causes promotion of charge carriers to higher energy states which shows as a red shift in the $1 \mathrm{~S}_{3 / 2} \rightarrow 1 \mathrm{~S}_{\mathrm{e}}$ optical transition. Thermal expansion and electron-phonon coupling contributions are investigated using temperature photoluminescence spectroscopy. 


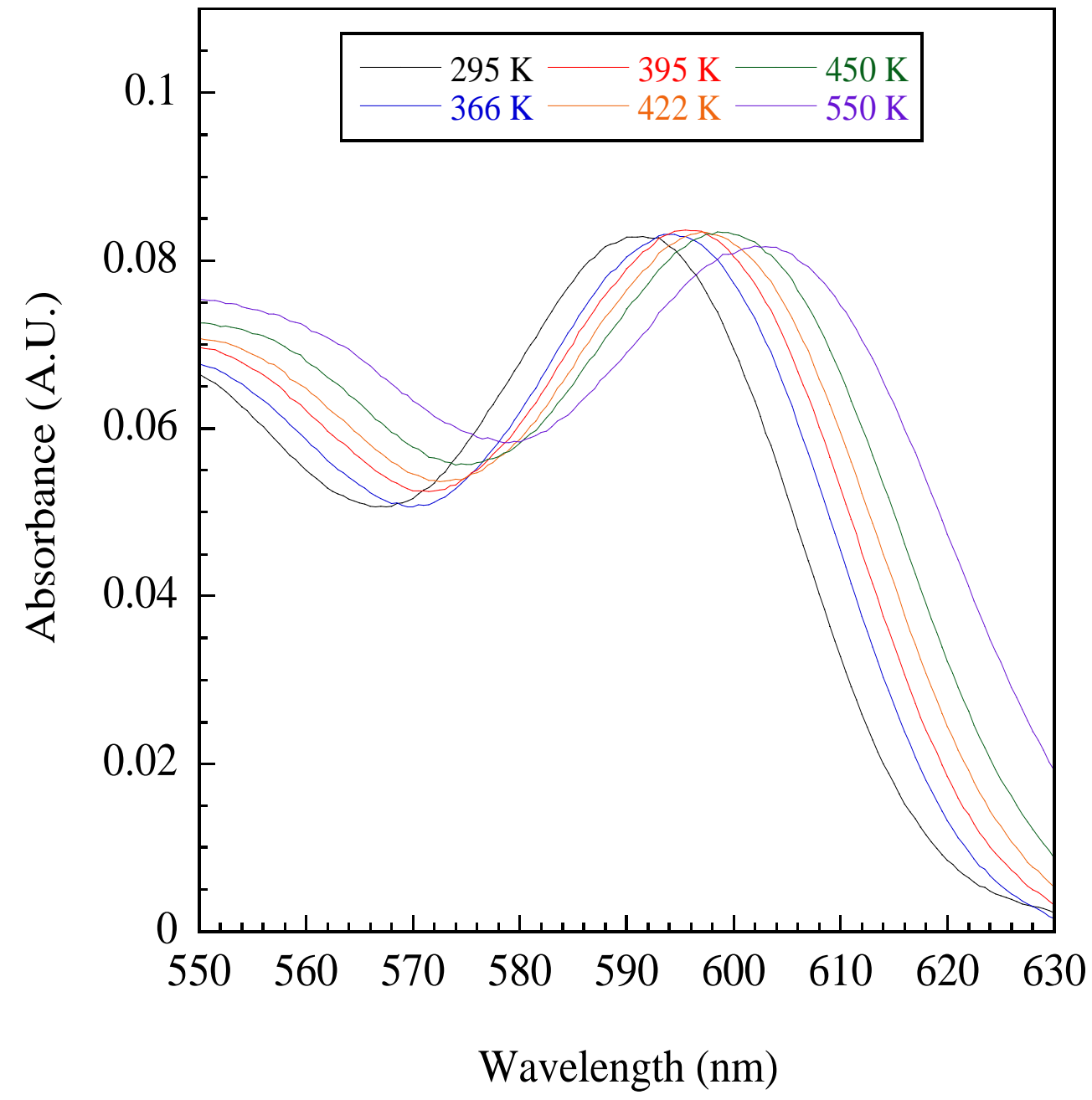

Figure 6-6: Temperature dependent absorption spectra from 295-550 K. 


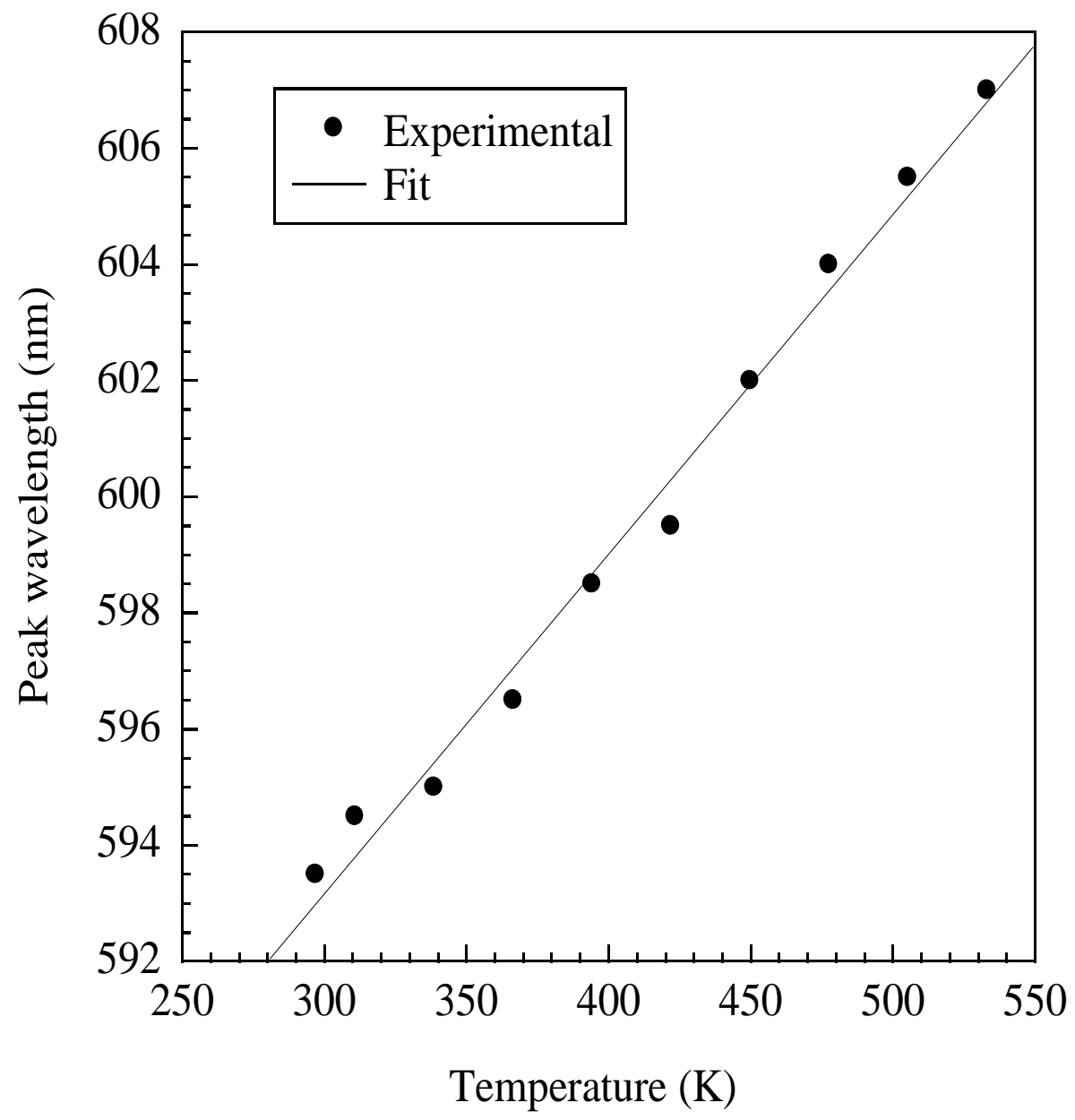

Figure 6-7: Variation in the first absorption peak wavelength with temperature. 


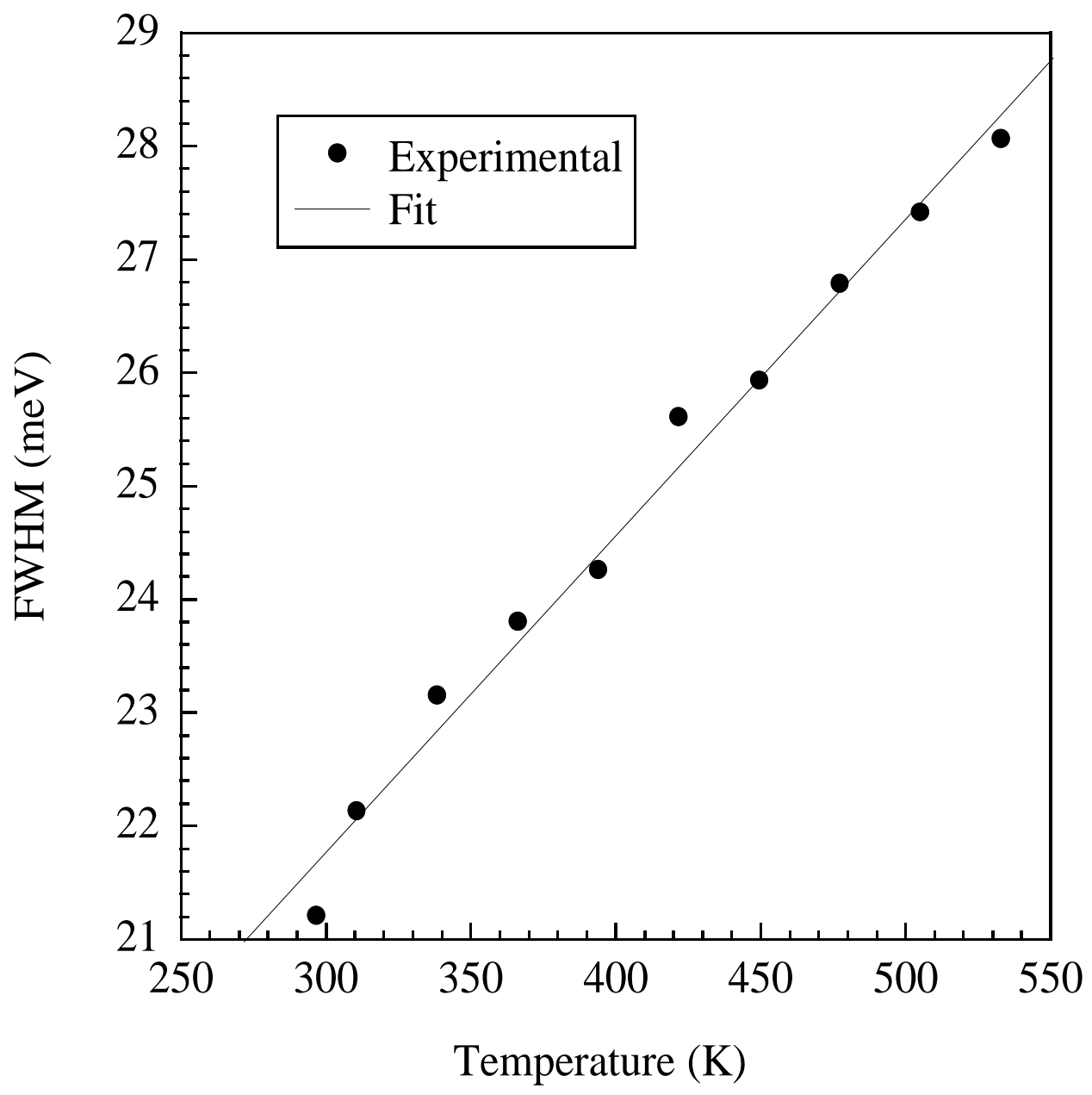

Figure 6-8: Full width at half maximum (FWHM) versus temperature. 


\subsection{Temperature Dependent Photoluminescence Spectroscopy}

The $\mathrm{CdSe} / \mathrm{ZnS}: \mathrm{SiO}_{2}$ quantum dot nanocomposite absorption and photoluminescence emission spectra are shown in Figure 6-9. The peak PL emission Stokes shifts by $11 \mathrm{~nm}$ with respect to the first QD exciton absorption peak. The peak emission wavelength is $608 \mathrm{~nm}(2.04 \mathrm{eV})$. Due to quantum confinement effects, electronic states in the conduction band (hole states in the valence band) become quantized as $E_{c 0}, E_{c 1}$ etc. $\left(E_{v 0}, E_{v 1}\right.$ etc.), where $E_{c 0}$ and $E_{v 0}$ denote the ground electron and hole state, respectively. Hence, from the effective mass approximation, the ground state energy level, $E_{1 s}$ is [99]:

$$
E_{1 s}=\frac{h^{2}}{2 m^{*} d^{2}}
$$

where, $h$ is Planck's constant, $d$ is the quantum dot diameter, and $m^{*}$ is the CdSe reduced mass. The effective band gap, $E_{g}^{e f f}$ of CdSe quantum dots is:

$$
E_{g}^{e f f}=E_{g}(b u l k)+\frac{h^{2}}{2 m^{*} d^{2}}
$$

where, $E_{g}($ bulk) is the band gap of bulk CdSe. The parameters in the approximation are: the CdSe bulk band gap is $1.742 \mathrm{eV}$ and the effective mass of electrons and holes are $0.13 \mathrm{~m}_{\mathrm{o}}$ and $0.45 \mathrm{~m}_{\mathrm{o}}$, respectively, where $\mathrm{m}_{\mathrm{o}}$ is the free electron mass. The approximate particle radius is calculated to be $3.6 \mathrm{~nm}$. Figure 6-10, is a schematic of $\mathrm{CdSe} / \mathrm{ZnS}: \mathrm{SiO}_{2}$ QD energy band structure. 


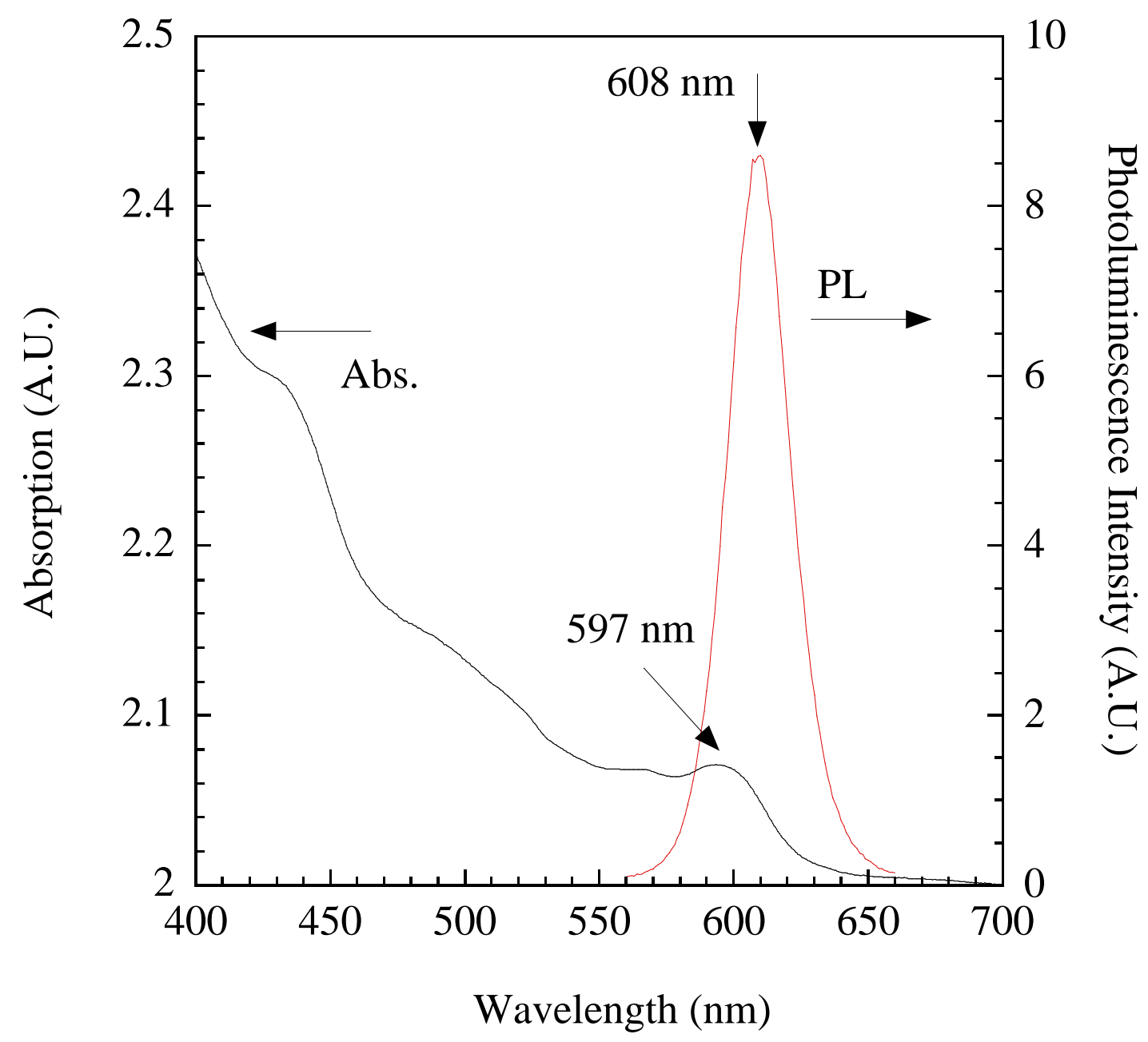

Figure 6-9: Normalized photoluminescence emission and absorption spectrum at $295 \mathrm{~K}$ for $50 \mu \mathrm{m}$ thick $\mathrm{CdSe} / \mathrm{ZnS}: \mathrm{SiO}_{2}$ nanocomposite film. 


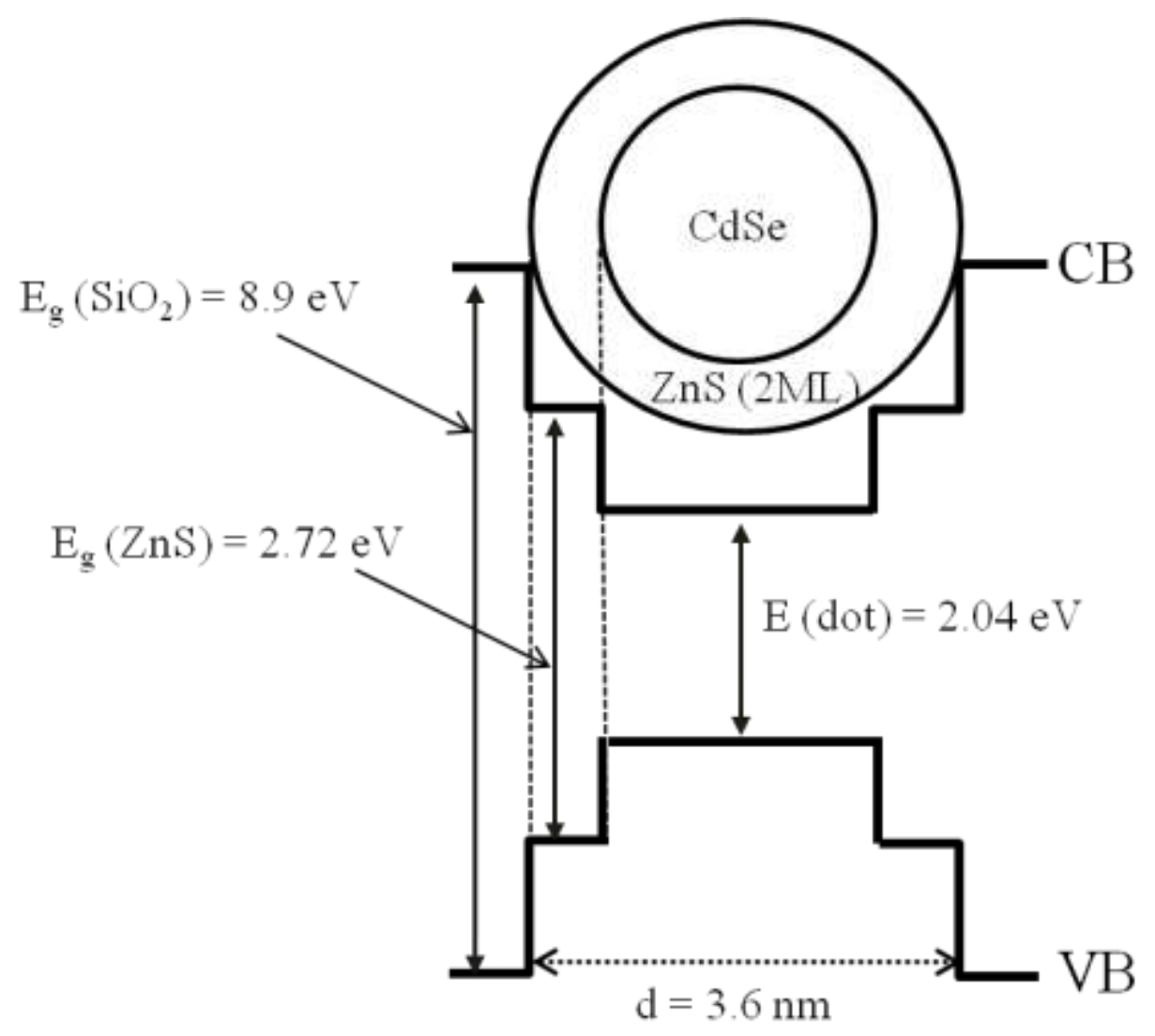

Figure 6-10: $\mathrm{CdSe} / \mathrm{ZnS}: \mathrm{SiO}_{2} \mathrm{QD}$ energy band diagram. 
The photoluminescence spectra were obtained in the range 550- $650 \mathrm{~nm}$, which covered the near-band edge region on $\mathrm{CdSe} / \mathrm{ZnS}: \mathrm{SiO}_{2}$ samples. The evolution of the PL emission spectra when the temperature is increased from 295 to $675 \mathrm{~K}$ is recorded in Figure 6-11a and b. Above $580 \mathrm{~K}$, the PL emission signal was weak but, measurable with some noise. The data were taken for a 2 hour continuous reversible heating process. The PL intensity band shifts and broadens with increasing temperature. Shown in Figure 6-12, a Lorentzian model yields a linear fit to the peak emission wavelength with temperature. The peak position is governed by different contributions to the room temperature PL emission compared to the PL emission in the high temperature regime. Heat expands the crystalline network of the QD material. The expansion causes a change in the energy band gap which is observed by a red-shift in the emission peak wavelength with increasing temperature. Similar to bulk semiconductors, the temperature-dependent variation in the energy band gap is due to thermally-induced lattice expansion and electron-phonon interactions $[69,100]$. The temperature dependent energy band gap, $\mathrm{E}_{\mathrm{g}}$ (T) in bulk semiconductors is given by the Varshni relation defined in Chapter 2, Equation 2.17:

$$
E_{g}(T)=E_{g}(0)-\frac{\alpha T^{2}}{T+\beta}
$$

where, $E_{g}(0)$ is the energy band gap at $0 \mathrm{~K}, \alpha$ and $\beta$ are the Varshni's coefficients. Varshni's relation has been extended to semiconductor quantum dots $[28,70,71]$. 


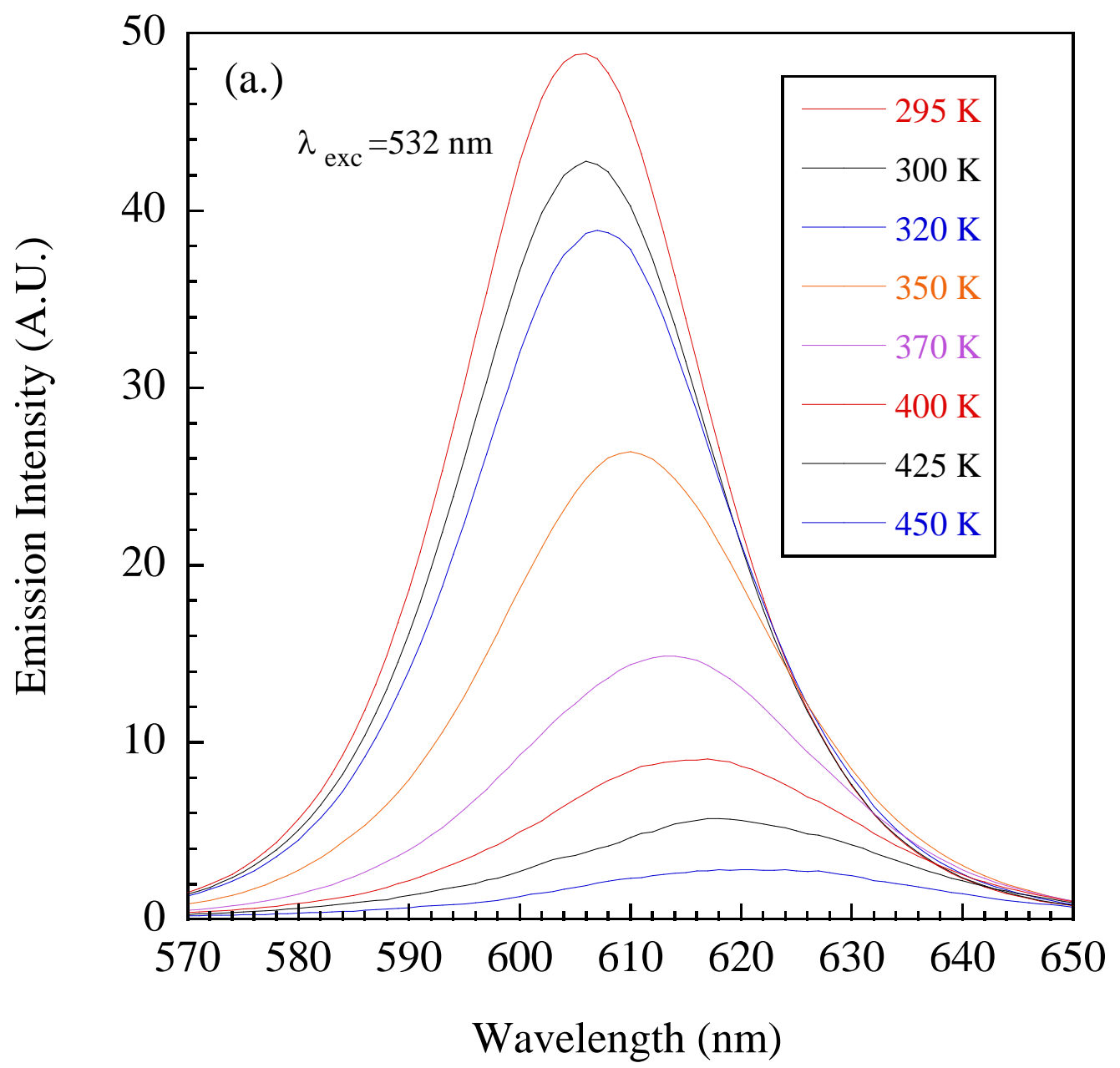




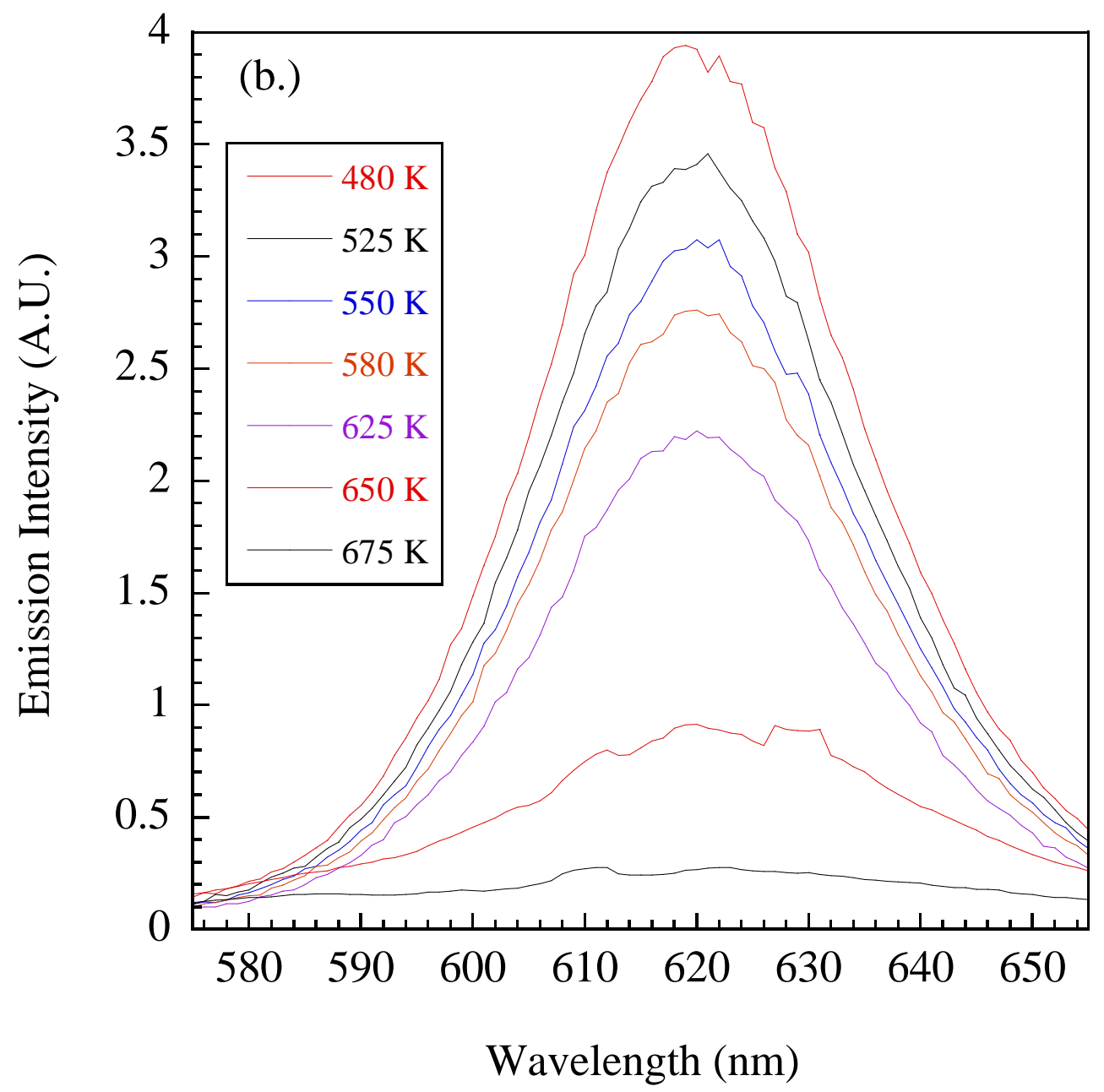

Figure 6-11: (a.) $\mathrm{CdSe} / \mathrm{ZnS}: \mathrm{SiO}_{2}$ photoluminescence emission measured as a function of temperature and (b.) Ultra-high temperature $\mathrm{CdSe} / \mathrm{ZnS}: \mathrm{SiO}_{2} \mathrm{PL}$ signal from $450-675 \mathrm{~K}$. 


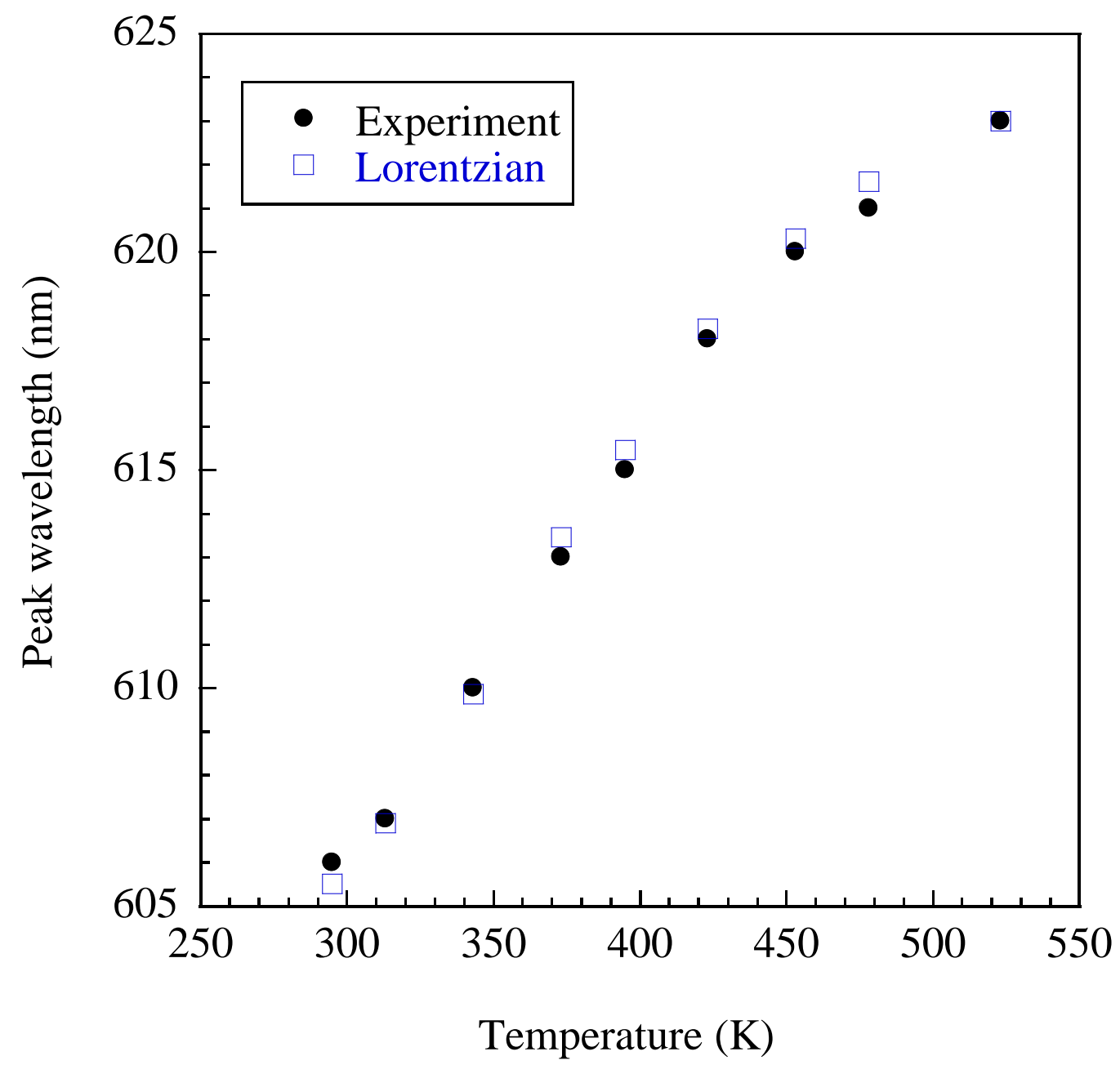

Figure 6-12: $\mathrm{CdSe} / \mathrm{ZnS}: \mathrm{SiO}_{2}$ photoluminescence emission peak wavelength measured as a function of temperature. 
Results on the extension of this empirical relation to semiconductor quantum dots are referenced in this work and are used as a benchmark comparison for the new $\mathrm{CdSe} / \mathrm{ZnS}: \mathrm{SiO}_{2}$ QD nanocomposite material development in this research. Table 6-3 compares the fit parameters for the $\mathrm{CdSe} / \mathrm{ZnS}: \mathrm{SiO}_{2}$ QDs to $\mathrm{CdSe}$ QDs embedded in polystyrene (PS), CdSe QDs embedded into a UV resin [28, 70, 71]. The fit parameters for bulk CdSe are included in the table. Shown in Figure 6-13, a best fit curve with $\alpha=$ $3.8 \times 10^{-4} \mathrm{eV} / \mathrm{K}$ and $\beta=150 \mathrm{~K}$ agrees well with experimental data up to $\sim 480 \mathrm{~K}$.

Table 6-3 Comparison of Varshni coefficients for CdSe/ZnS quantum dots.

\begin{tabular}{||c|c|c||c|c|}
\hline \hline Varshni Coefficient & $\begin{array}{c}\text { This study } \\
\text { CdSe/ZnS:SiO }\end{array}$ & $\begin{array}{c}\text { CdSe/ZnS:PS } \\
{[70]}\end{array}$ & $\begin{array}{c}\text { CdSe/ZnS:Resin } \\
{[71]}\end{array}$ & $\begin{array}{c}\text { CdSe bulk } \\
{[28]}\end{array}$ \\
\hline \hline$\alpha\left(10^{-4} \mathrm{eV} / \mathrm{K}\right)$ & $3.8 \pm 0.2$ & $3.2 \pm 0.2$ & $4.5 \pm 0.6$ & $2.8-4.1$ \\
$\beta(\mathrm{K})$ & $150 \pm 20$ & $220 \pm 30$ & $195 \pm 70$ & $181-315$ \\
$\mathrm{~T}(\mathrm{~K})$ & $293-550$ & $45-295$ & $10-295$ & 295 \\
\hline
\end{tabular}




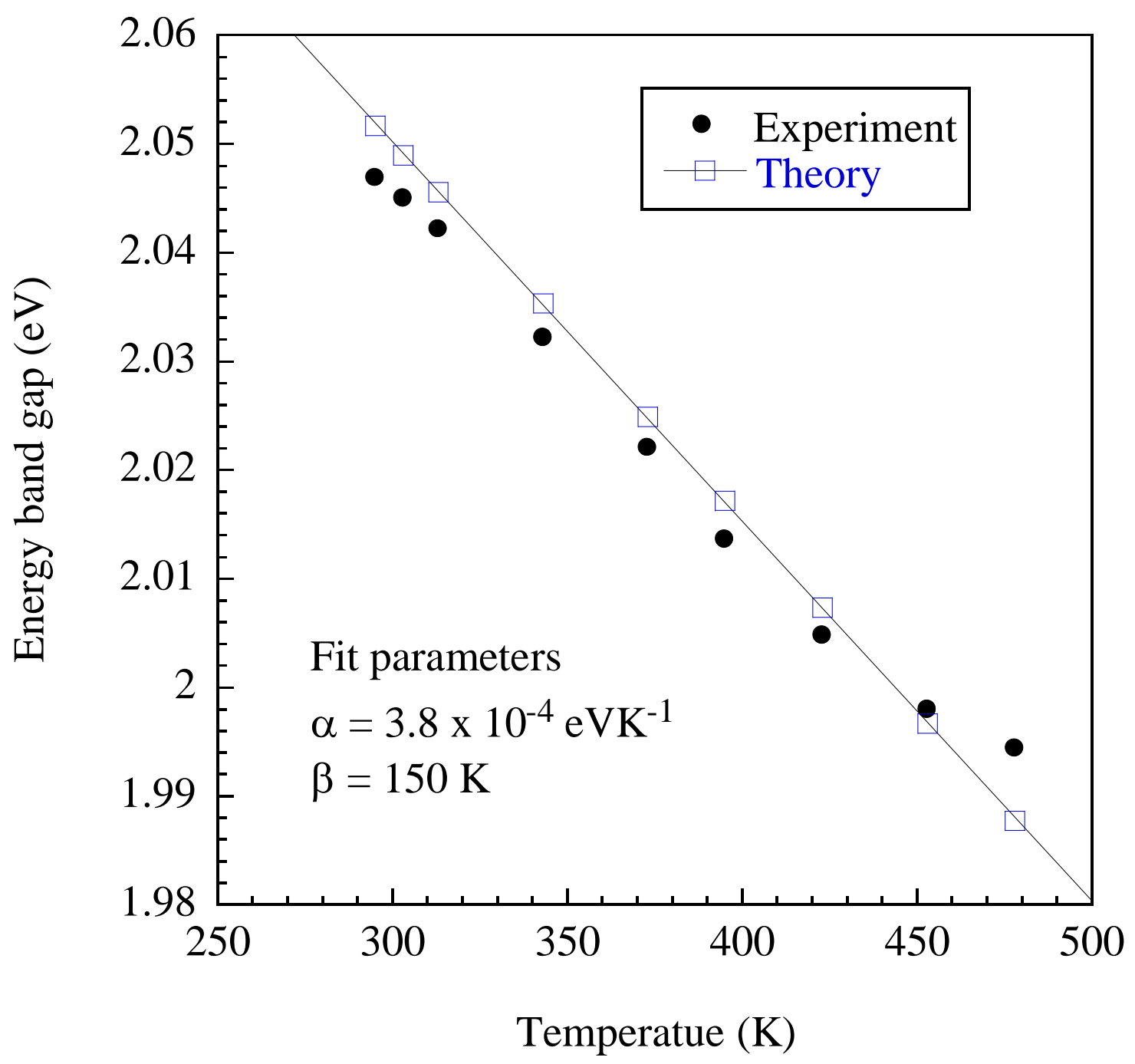

Figure 6-13: Temperature dependent energy band gap of $\mathrm{CdSe} / \mathrm{ZnS}: \mathrm{SiO}_{2} \mathrm{QD}$ film. 
The $\alpha$ parameter represents the linear shift in the band gap with respect to temperature. For $\alpha$ smaller than the $\alpha_{\text {bulk }}$ implies that variation in the energy band gap is weaker for the discrete states in QDs compared to bulk CB-VB transitions. This indicates that QDs have better thermal stability than bulk crystals. A significantly lower $\beta$ indicates increased coupling of the transition to lower energy acoustic phonons and reduced coupling to longitudinal optical phonons. Insight into exciton-phonon coupling is gained from the emission spectrum full width at half maximum (FWHM).

Two factors permit the extension to high temperatures. First, the $\mathrm{ZnS}$ shell passivation enhances the quantum yield. Secondly, the high temperature resistant $\mathrm{SiO}_{2}$ dielectric matrix reduces non-radiative recombination centers on the QD surface and protects the QDs to enable PL signal measurement in the ultra-high temperature regime. Above $625 \mathrm{~K}$, the PL intensity rapidly decreases (see Figure 6-11 b). This means that there are a significant amount of thermally-induced non-radiative traps at the QD-matrix interface which cause thermal quenching of the emission. In the $\mathrm{CdSe} / \mathrm{ZnS}: \mathrm{SiO}_{2}$ quantum dot structure, the QD-surface interface states play a very significant role in the emission, especially the PL emission in the ultra-high temperature regime. Large thermal expansion of the $\mathrm{SiO}_{2}$ lattice induces strain in the $\mathrm{CdSe} / \mathrm{ZnS}$ quantum dots which appears to alter the linear response of the $\mathrm{CdSe} / \mathrm{ZnS}: \mathrm{SiO}_{2}$ energy band gap with temperature. Shown in Figure 6-14, the QD energy band gap asymptotes due to heat, lattice vibrations, and thermally-induced strain in the $\mathrm{SiO}_{2}$ matrix. A few studies on strain sensing with CdSe QDs have recently appeared in the literature [101, 102]. However, at the time of this thesis writing, no single study on the thermally induced strain in colloidal $\mathrm{CdSe} / \mathrm{ZnS}$ quantum dots was found in the literature. 


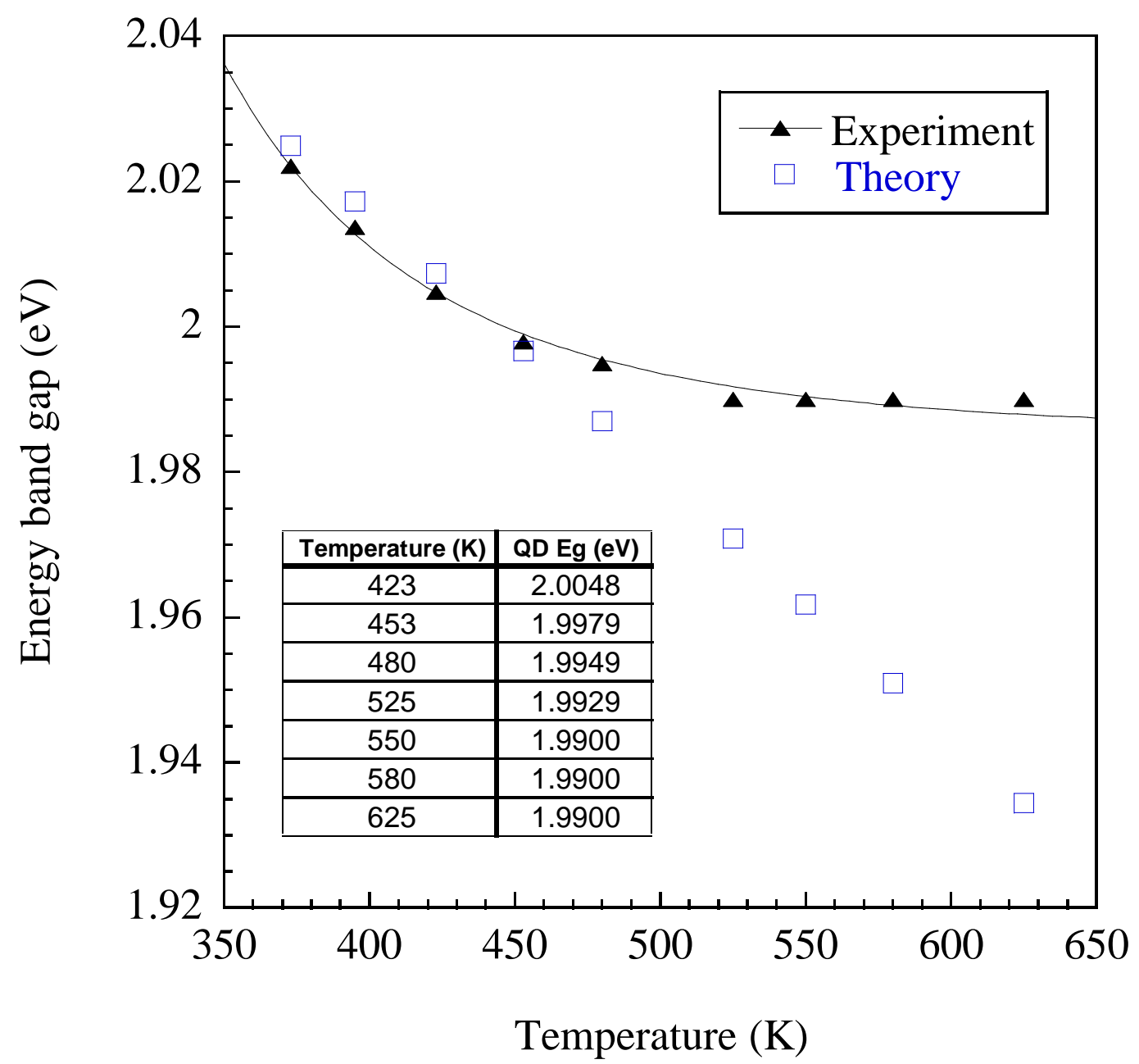

Figure 6-14: Temperature dependent energy band gap of $\mathrm{CdSe} / \mathrm{ZnS}: \mathrm{SiO}_{2} \mathrm{QD}$ film from 423- $625 \mathrm{~K}$. 
Figure 6-15 reports the variation in full width at half maximum (FWHM) with temperature. The FWHM broadens with increasing temperature. The width of the band is due to electron-phonon interactions. In general, line broadening is composed of inhomogeneous and homogeneous terms. The homogeneous term can be obtained at higher temperatures by fitting the PL spectra with a complex Voigt function. To simplify the calculation, the emission peak was fit to a Lorentzian function for all temperatures. The carrier-phonon processes (exciton scattering with acoustic and longitudinal optical (LO) phonons) that result in the broadening of the FWHM, $\Gamma$ with increasing temperature can be defined by Eqn. 2.18 in Chapter 2 (shown in Figure 6-15). $\quad \mathrm{E}_{\mathrm{LO}}$ is taken as 25 meV from Raman spectra on CdSe quantum dots in previous reports [103, 104]. A good agreement between the experimental data and the best fit curve is obtained for $\Gamma_{\mathrm{inh}}=52.5$ $\mathrm{meV}, \Gamma_{\mathrm{LO}}=50 \pm 1 \mathrm{meV}$, and $\gamma_{\mathrm{AC}}=0.031 \mathrm{meV} / \mathrm{K}$. The exciton-LO phonon coupling coefficient is significantly smaller than that of $100 \mathrm{meV}$ in bulk CdSe [70]. The theory of exciton-phonon interaction in quantum dots predicts a reduced exciton-LO phonon coupling and an enhanced exciton-acoustic phonon interaction. These data are consistent with theoretical predictions of a strong increase in the coupling with acoustic phonons in zero-dimensional material systems. 


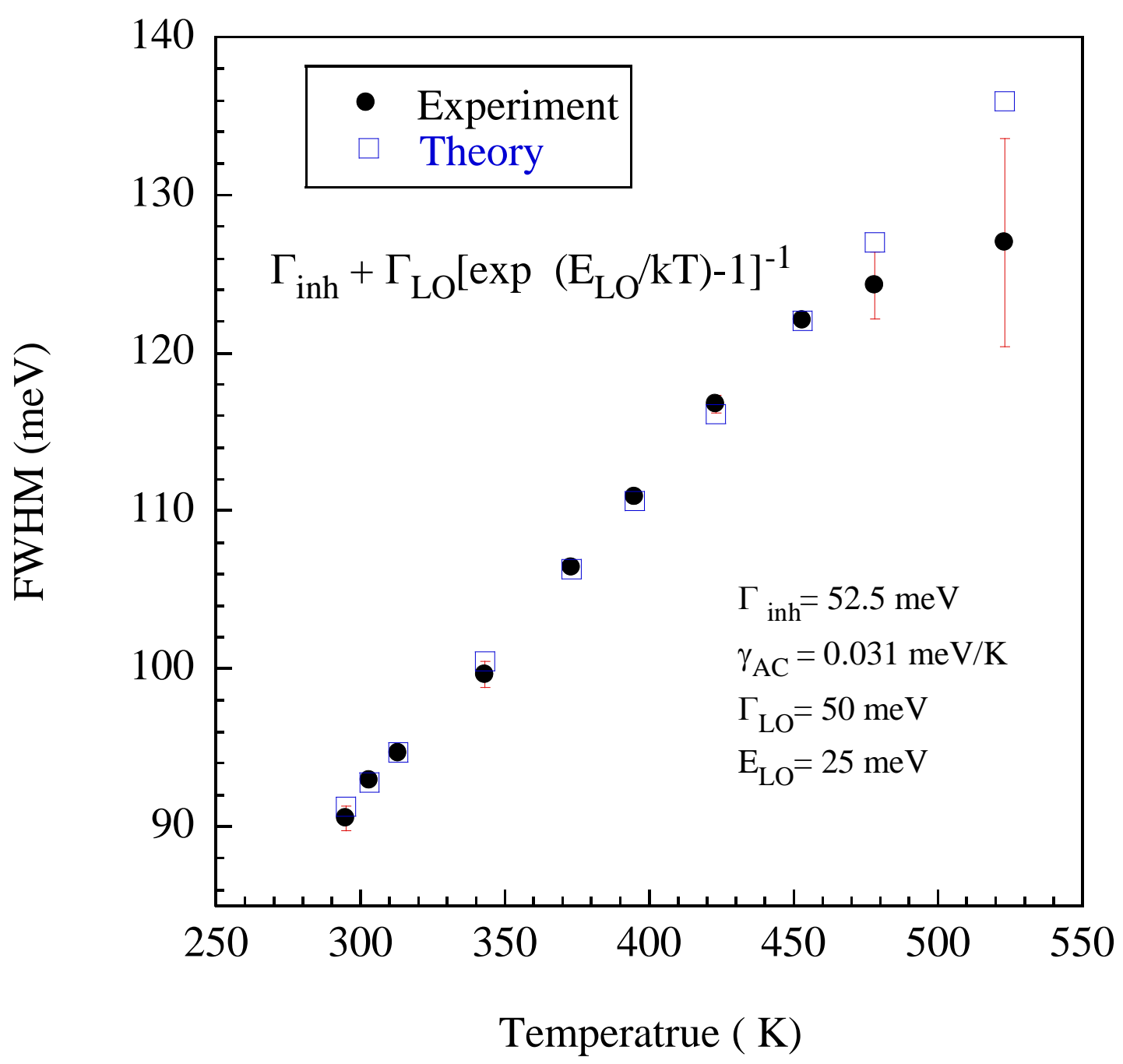

Figure 6-15: Variation in the full width at half maximum with respect to temperature. 
The variation in PL intensity with temperature is shown in Figure 6-16. The PL intensity decreased linearly from room temperature up to $500 \mathrm{~K}$. A faster, exponential decrease is observed from $526-650 \mathrm{~K}$. The data in Fig. 6-16 is approximated by an exponential with a correlation factor of $\mathrm{R}^{2}=0.985$. In general, the PL intensity of quantum dots exponentially decreases with increasing temperature due to thermal quenching. Thermal quenching in core and core/shell dots is attributed to carrier trapping by surface defect states (traps) and thermal escape due to scattering with multiple LO phonons. Furthermore, the ligand shell and the matrix also influence the temperature dependence of the PL intensity. For $\mathrm{CdSe} / \mathrm{ZnS}: \mathrm{SiO}_{2}$ QDs, surface passivation with higher-bandgap quantum shell $\mathrm{ZnS}$ electronically isolates the core for stable, maximized PL emission. ZnS buries the CdSe in a potential energy well, concentrating the charge carriers within the nanocrystal core. The $\mathrm{S}^{2-}$ with a much lower oxidation potential than $\mathrm{Se}^{2-}$ results in a higher threshold to photoxidative degradation and surface defect formation. When embedded into high temperature resistant $\mathrm{SiO}_{2}$ dielectric, extension of $\mathrm{CdSe}(\mathrm{ZnS})$ quantum dots into the high temperature sensing regime is accomplished. 


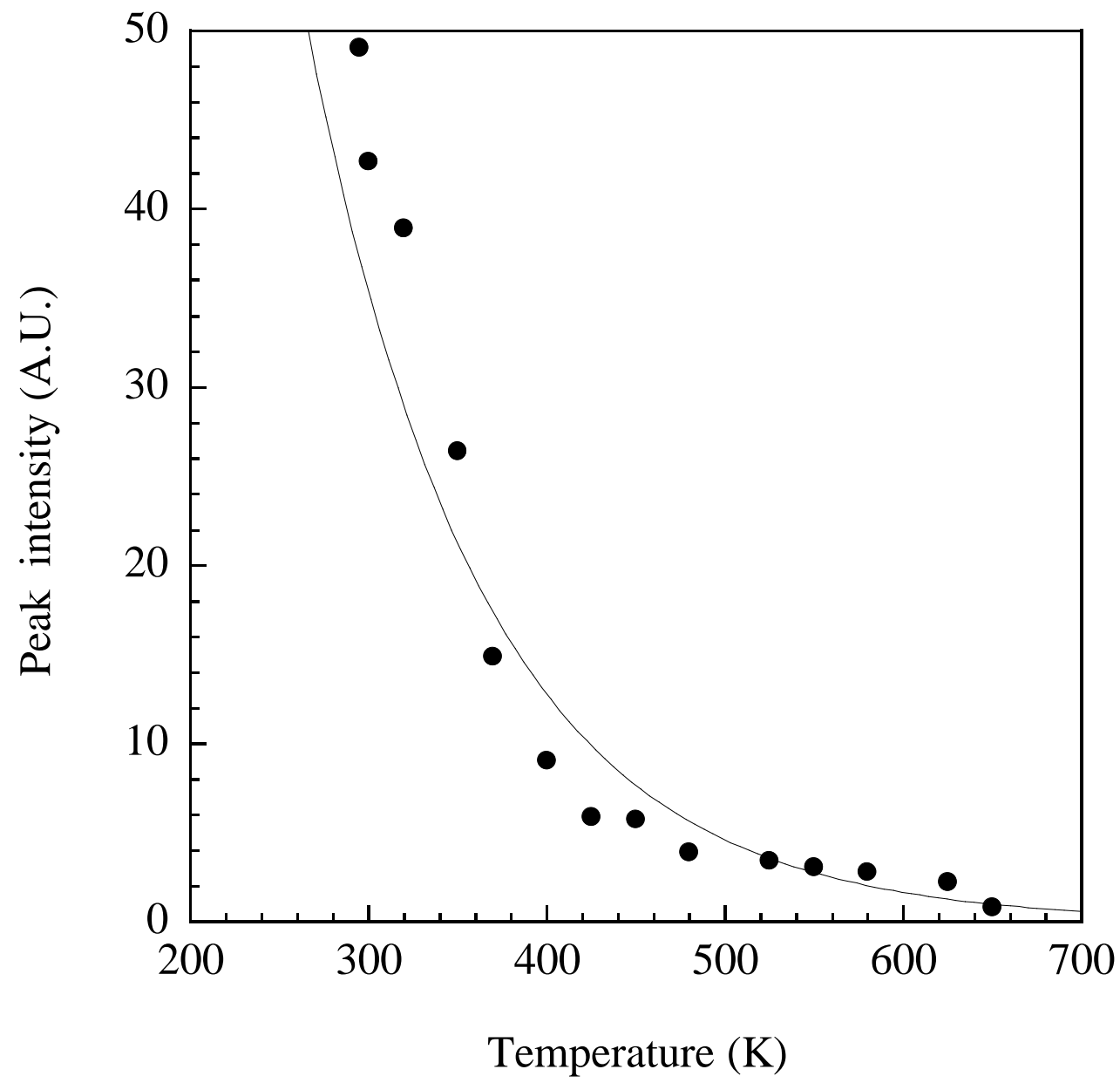

Figure 6-16: Variation in the PL intensity with respect to temperature. 


\subsection{Temperature Dependent Lifetime Analysis}

The first exciton absorption band for the $\mathrm{CdSe} / \mathrm{ZnS}: \mathrm{SiO}_{2}$ nanocomposite film extends from 597 to $570 \mathrm{~nm}$ (see Section 6.1). The oscillator strength represents the strength of the transition. The oscillator strength $f$ was calculated by integrating $\sigma_{\mathrm{a}}$ over the first exciton transition absorption band [87]

$$
f=\frac{4 m \varepsilon_{o} c}{e^{2}} \int_{v_{1}}^{v_{2}} \sigma_{a}(v) d v
$$

where, $v_{1}$ and $v_{2}$ are the initial and end frequencies of the first exciton absorption band, $m$ is the mass of an electron, $c$ is the speed of light, $\varepsilon_{\mathrm{o}}$ is the permittivity of free space, and e denotes the charge of an electron. The integral of the absorption cross section from 295 to $550 \mathrm{~K}$ was calculated over $v_{1}=5.00 \times 10^{14} \mathrm{~Hz}$ and $v_{2}=5.25 \times 10^{14} \mathrm{~Hz}$. The radiative lifetime $\tau$ was extracted using

$$
\tau=\left[\frac{8 \pi}{\lambda^{2}} \int_{v_{1}}^{v_{2}} \sigma_{a}(v) d v\right]^{-1}
$$

where $\lambda$ is the peak wavelength of the $1 S_{3 / 2} \rightarrow 1 S_{e}$ transition. A Lorentzian function was fitted to the first exciton band and then the integral was performed over the lineshape. Table 6.4 gives the temperature dependent oscillator strength and lifetimes for $\mathrm{CdSe} / \mathrm{ZnS}: \mathrm{SiO}_{2}$ QD nanocomposite film. The radiative lifetime calculated from absorption cross section is expected to decrease with temperature. A $20 \%$ decrease in the radiative lifetime is calculated for the $\mathrm{CdSe} / \mathrm{ZnS}: \mathrm{SiO}_{2}$ QD material system. QD PL and radiative decay is a complex process that involves contributions from carrier 
trapping, e-h interactions, local environment responses. These contributions to the quantum efficiency being consider to further characterize and extend QD thermal sensing regime [85].

Table 6.4 Oscillator strength and radiative lifetime of $\mathrm{CdSe} / \mathrm{ZnS}: \mathrm{SiO}_{2} \mathrm{QD}$ film $1 \mathrm{~S}_{3 / 2} \rightarrow 1 \mathrm{~S}_{\mathrm{e}}$ first absorption peak.

\begin{tabular}{|c|c|c||c|c|}
\hline Temperature (K) & $\begin{array}{c}1 \mathrm{~S}_{3 / 2} \rightarrow 1 \mathrm{~S}_{\mathrm{e}} \text { band } \\
(\mathrm{nm})\end{array}$ & $\begin{array}{c}1 \mathrm{~S}_{3 / 2} \rightarrow 1 \mathrm{~S}_{\mathrm{e}} \\
\text { peak } \\
(\mathrm{nm})\end{array}$ & Oscillator Strength & $\begin{array}{c}\text { Radiative Lifetime } \\
(\mathrm{ns})\end{array}$ \\
\hline \hline 295 & $570-597$ & 593.5 & 4.3 & 101.8 \\
\hline 366 & $570-597$ & 594.5 & 3.9 & 95.6 \\
\hline 395 & $570-597$ & 595.0 & 3.98 & 92 \\
\hline 422 & $570-597$ & 598.5 & 3.87 & 85.53 \\
\hline 450 & $570-597$ & 599.5 & 3.67 & 80.2 \\
\hline 550 & $570-597$ & 602.0 & 3.66 & \\
\hline
\end{tabular}




\section{Chapter 7 \\ FIBER OPTIC QD-LUMINESCENCE THERMAL SENSOR}

"Nothing can dim the light which shines from within."

Maya Angelou

\subsection{Introduction}

Luminescence thermometers measure the temperature variations using the spectral and temporal modes from active center of select materials. In general, a UV-vis light beam is focused into an optical fiber and excites rare earth elements or transition metal ions in a bulk crystal mounted to the end of fiber. The emission light is guided through fiber and detected for single point temperature measurement. Luminescence thermometry offers many advantages over conventional temperature transducers. No probe such as the highly conductive metals used with thermocouples and resistance thermometers are required to conduct heat to or away from the surface resulting in temperature measurement error. In addition, optical signals are not perturbed by electromagnetic fields which can make electronic thermometry near impossible. Fiber optic luminescence-based thermal sensors enable remote sensing in locations that are

difficult to access. These fundamental differences give fiber optic fluorescence thermometry the following advantages over traditional thermometers:

1. Electrical, Magnetic, and Electromagnetic Immunity. The probe materials are good electrical insulators. They do not introduce electrical shorting paths or cause 
electrical problems. The probes do not absorb significant amounts of electromagnetic radiation or become heated by stray fields.

2. Small Sensor Size. The small size also means small thermal mass.

3. Rapid Response. The optical sensor exhibits very rapid thermal response.

4. Capability of Remote Measurement. The electrical, chemical, and thermal inertness allow for long-term location of the sensor inside complex equipment.

In this chapter, the benefits of $\mathrm{CdSe} / \mathrm{ZnS}$ QDs such as fast response time are combined with the optical fiber advantages sited above for a high temperature thermal sensor demonstration. Section 7.2 presents the sensor design and fabrication processes. Section 7.3 presents the experimental setup and sensor characterization data.

\subsection{Fiber Optic Based Quantum Dot Thermal Sensor Design and Fabrication}

A diagram of the thermal sensor design is shown in Figure 7-1. The sensor was fabricated using high temperature resistant bare 100/140 $\mu \mathrm{m}$ core/clad diameter hard clad silica (HCS) optical fiber. The same wet chemistry that was developed for the $\mathrm{CdSe} / \mathrm{ZnS}: \mathrm{SiO}_{2}$ QD nanocomposite film fabrication (Chapter 3) was used to coat the end tip of the optical fiber. Coating the fiber end tip with the $\mathrm{CdSe} / \mathrm{ZnS}: \mathrm{SiO}_{2}$ nanocomposite solution was a nontrivial task. First, the plastic cladding of one end of the fiber was stripped. Then the fiber was cleaved using a splicing machine. Repeated trials at straightedge cleaving of the optical fiber did not give a uniform deposit of QDs and also resulted 
in weak PL emission. The $\mathrm{CdSe} / \mathrm{ZnS}: \mathrm{SiO}_{2}$ solution dried at the periphery of the end-tip, similar to the initial QD sol-gel film fabrication on quartz wafers discussed in Chapter 3. Cleaving the end tip at a $45^{\circ}$ angle gave a asymmetrical surface on which to cast the QDs yielding a more uniform deposit. Figure 7-2 a-f shows the QD-coated optical fiber under 100, 200 and 500X magnification. The images were captured by mounting the $\mathrm{CdSe} / \mathrm{ZnS}: \mathrm{SiO}_{2}$ QD fiber optic sensor on the microscope stage as shown in Figure 7-3. The dots are made visible by a UV lamp (shown in the right-hand side of the figure). The $\mathrm{CdSe} / \mathrm{ZnS}: \mathrm{SiO}_{2}$ QD fiber optic sensor was then mounted into the experimental setup and characterized.

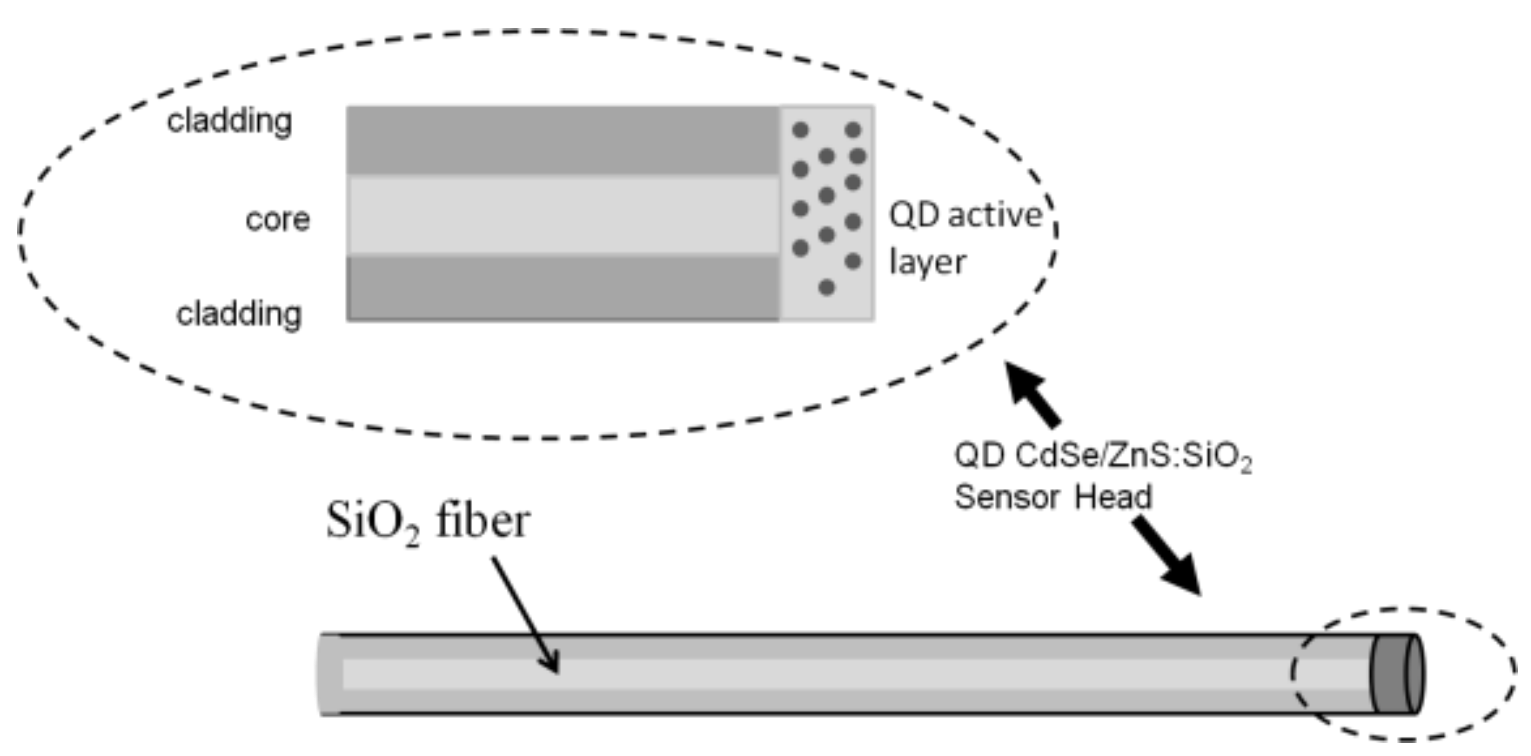

Figure 7-1: Compact $\mathrm{CdSe} / \mathrm{ZnS}: \mathrm{SiO}_{2}$ thermal sensor design. 

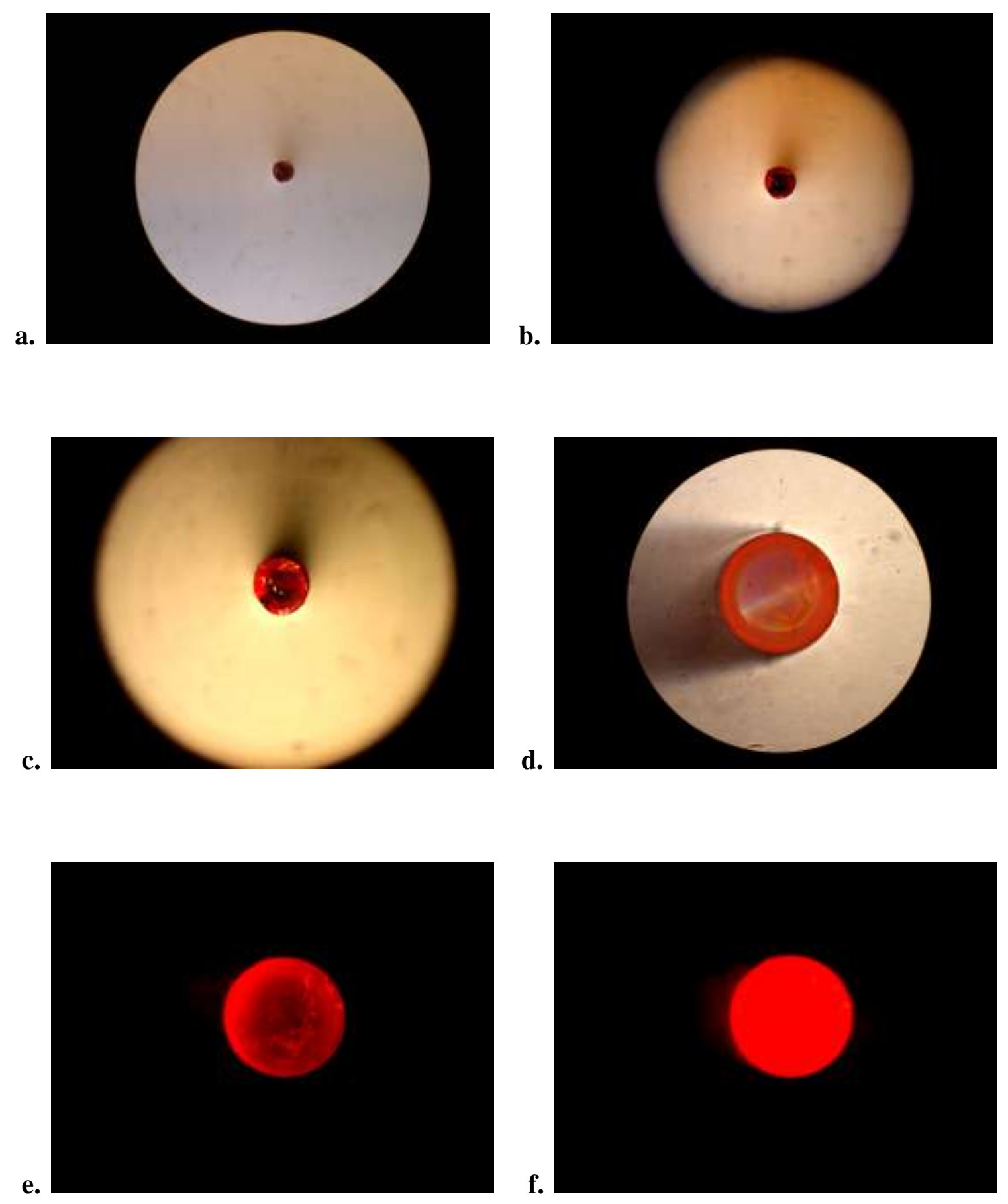

Figure 7-2: $\mathrm{CdSe} / \mathrm{ZnS}: \mathrm{SiO}_{2}$ end tip coated optical fiber imaged under (a.) 100X, (b.) and (c.) 200X, and (d-f) 500X microscope objective. Images were acquired with the microscope in (a.) transmission mode, (b-e.) transmission + UV mode, and (f.) UV mode. UV-mode means that the fiber was under UV illumination during imaging. 


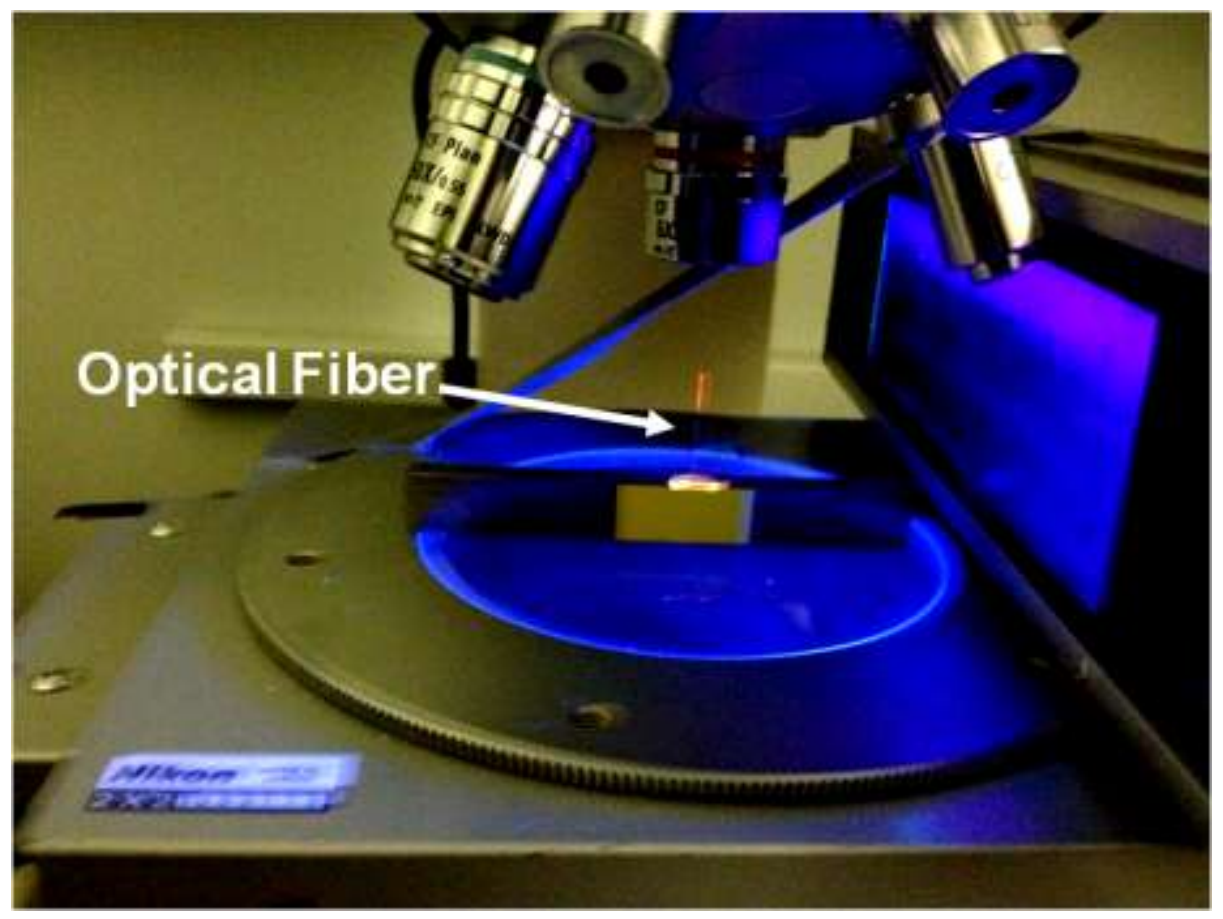

Figure 7-3: $\mathrm{CdSe} / \mathrm{ZnS}: \mathrm{SiO}_{2} \mathrm{QD}$ coated optical fiber on optical microscope stage. 


\subsection{Fiber Optic Based QD-Thermal Sensor Characterization}

\subsubsection{Instrumentation}

The sensor was mounted into the experimental setup shown in Figure 7-4. A VD III-A-532 laser was used to excite the QDs at the end-tip of the optical fiber. A 60x objective lens was used to couple the laser light into the fiber. The length of the fiber was $12.7 \mathrm{~cm}(5 \mathrm{in})$. It was found that longer QD-doped optical fibers produced low emission. The photoluminescence was collected at the fiber output and coupled with a second objective lens, into a multimode fiber connected to an Ocean Optics HR 4000 spectrometer. A $560 \mathrm{~nm}$ colored filter paper removed the pump beam.

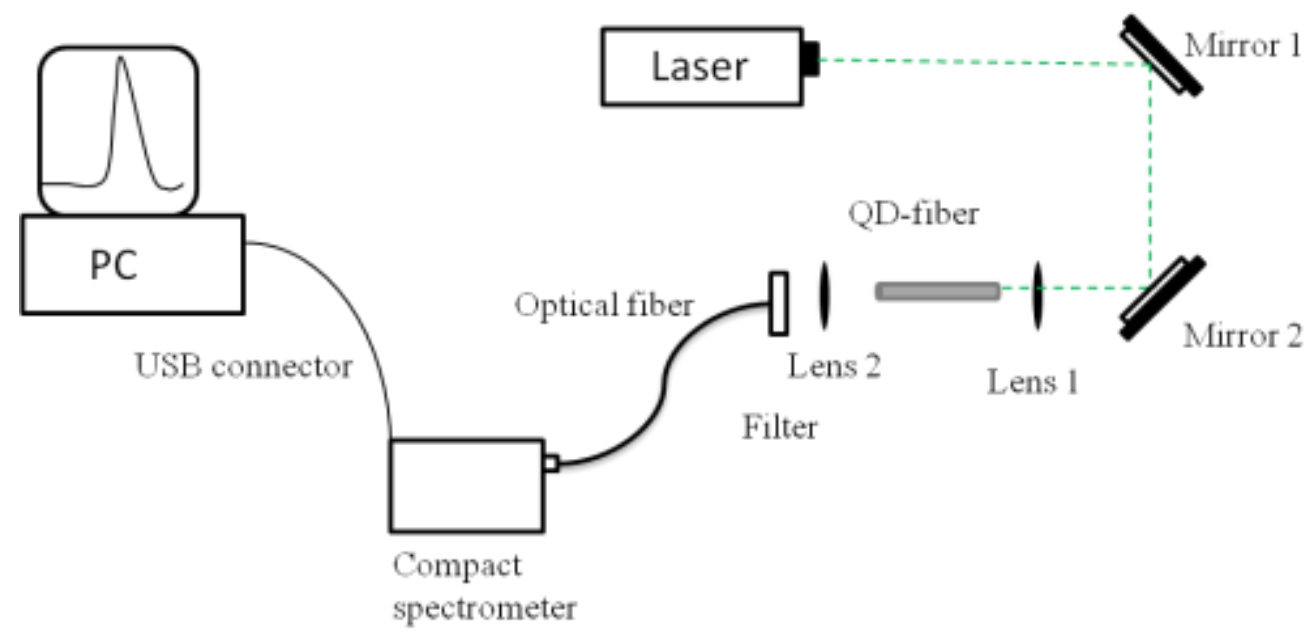

Figure 7-4: Experimental setup used to characterize thermal sensor. 


\subsubsection{Characterization}

Figure 7-5 shows the emission spectra for several temperatures. At $\mathrm{T}=295 \mathrm{~K}$, the emission peak was at $609 \mathrm{~nm}$ with a FWHM of $22.81 \mathrm{~nm}$. The spectrometer records the QD emission spectrum. As shown in Figure 7-6, the peak intensity decreases when the temperature rises. To simulate a real-time sensor, data was taken under a rapid ramp thermal cycle from 295-6205 K back down to room temperature. It took on average of $30 \mathrm{~s}$ to reach each set point. Figure 7-7 shows the spectral wavelength shift with temperature under high temperature thermal cycle. The PL emission wavelength increases linearly with ramp up and decreases linearly during the cool down tests. The sensor recovers the initial wavelength shift after thermal cycle. The sensor sensitivity is $0.11 \mathrm{~nm} /{ }^{\circ} \mathrm{C}$. However, the PL intensity does not completely recover after thermal cycling. The PL intensity recovery improves after two heating cycles compared to a single heating and cooling cycle. Compaction of the $\mathrm{SiO}_{2}$ matrix after the heat cycles helps in the signal recovery and sensor stability. Repeated trial revealed that the QD PL emission remained stable for at least a few days, demonstrating a practical QDluminescence based high temperature sensor. The $\mathrm{CdSe} / \mathrm{ZnS}: \mathrm{SiO}_{2}$ QD luminescencebased thermal sensor is more sensitive than conventional luminescence sensor based on bulk crystals. Table 7.1 compares the new $\mathrm{CdSe} / \mathrm{ZnS}: \mathrm{SiO}_{2}$ quantum dot material system to a conventional alexandrite bulk crystal sensor characterized in Appendix C. 


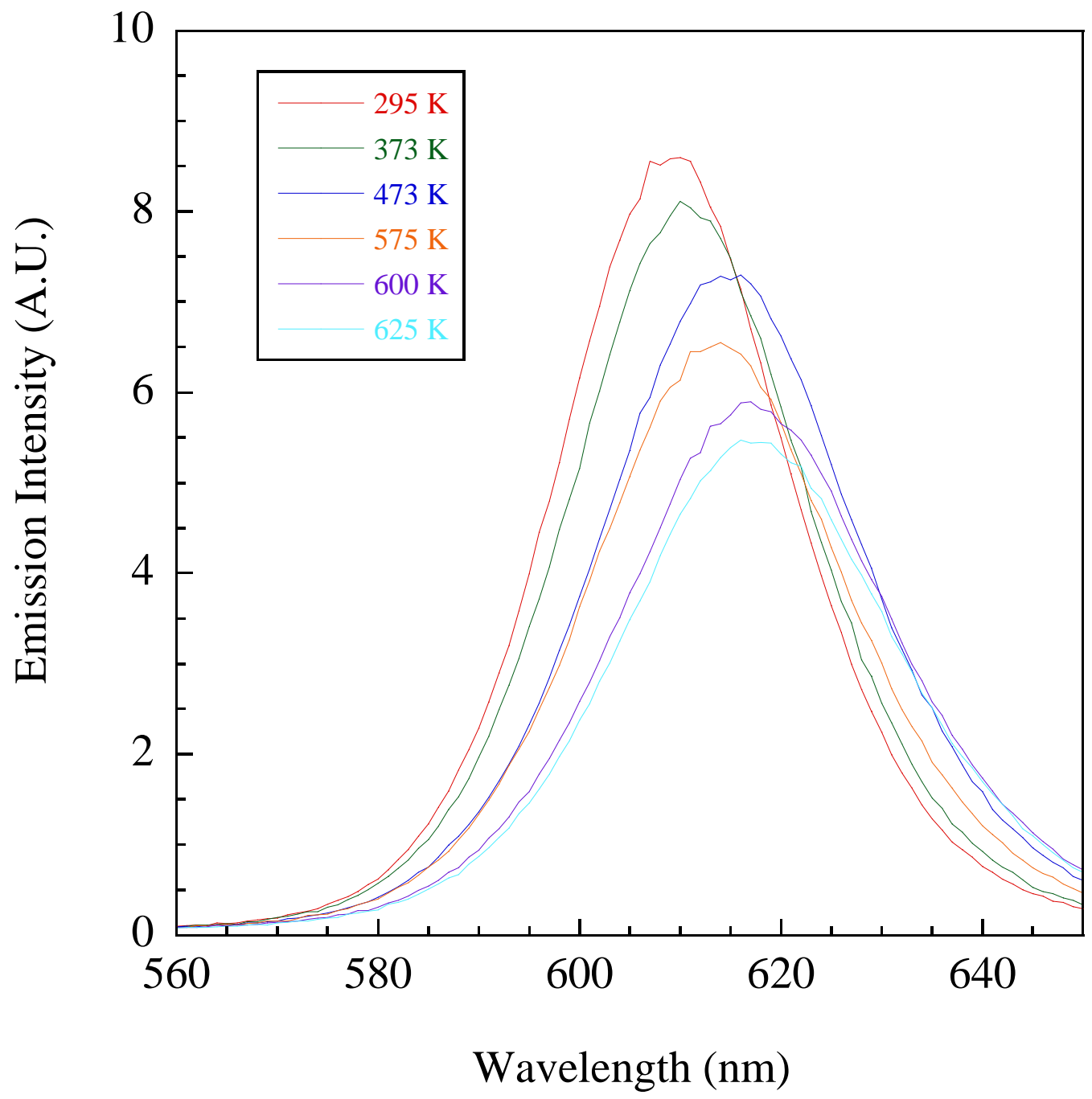

Figure 7-5: PL signal output for QD-fiber optic sensor from 295- $625 \mathrm{~K}$. 


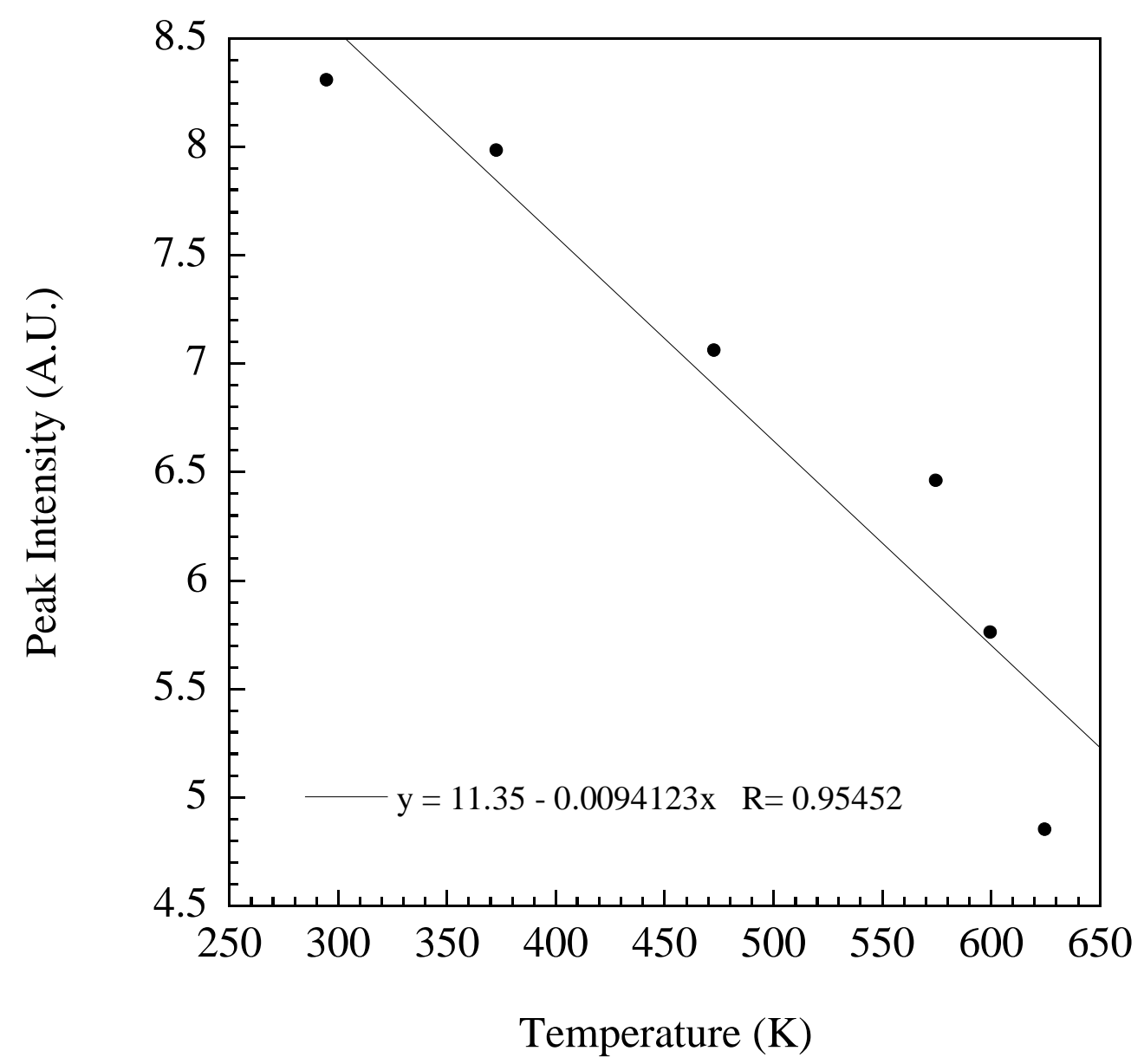

Figure 7-6: Peak intensity versus temperature for $\mathrm{CdSe} / \mathrm{ZnS}: \mathrm{SiO}_{2}$ thermal sensor. 


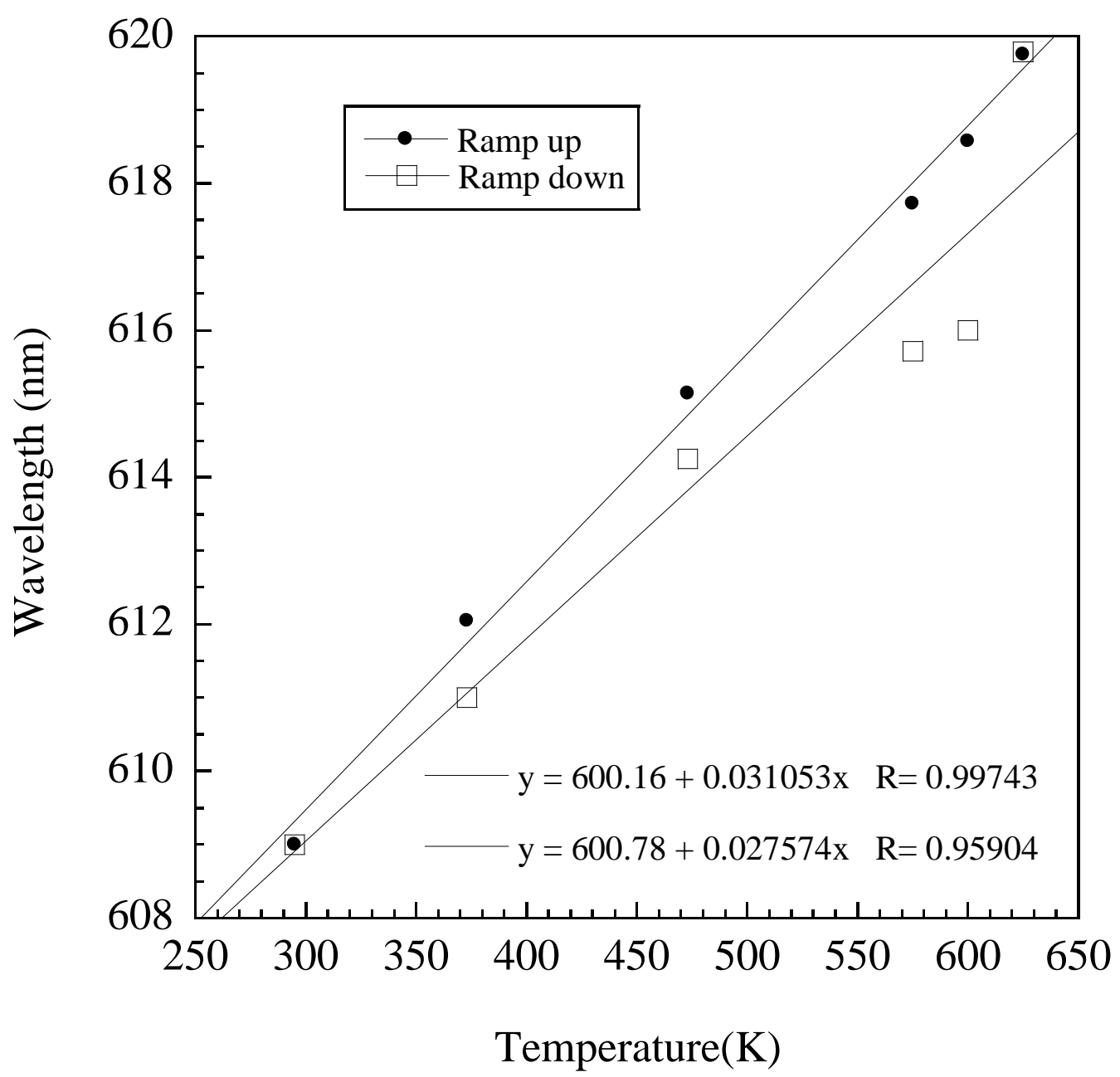

Figure 7-7: Peak wavelength versus temperature for $\mathrm{CdSe} / \mathrm{ZnS}: \mathrm{SiO}_{2}$ thermal sensor. Heating (circles) and cool down (squares). 
Table 7.1 Comparison of thermal QD sensor to bulk crystal response.

\begin{tabular}{|c|c|c|}
\hline Parameter & $* \mathrm{BeAl}_{2} \mathrm{O}_{4}: \mathrm{Cr}^{3+}$ & $\bar{C} \mathrm{CdSe}(\mathrm{ZnS}): \mathrm{SiO}_{2}$ \\
\hline Range & $22^{\circ} \mathrm{C}-230^{\circ} \mathrm{C}$ & $22^{\circ} \mathrm{C}-380^{\circ} \mathrm{C}$ \\
\hline$\Delta \lambda /{ }^{\circ} \mathrm{C}$ & $\begin{array}{l}\mathrm{R}_{1} \text { line }=0.01 \\
\mathrm{~nm} /{ }^{\circ} \mathrm{C} \\
\mathrm{R}_{2} \text { line }=1.43 \\
\mathrm{~nm} /{ }^{\circ} \mathrm{C}\end{array}$ & $0.11 \mathrm{~nm} /{ }^{\circ} \mathrm{C}$ \\
\hline$\Delta \mathrm{I} /{ }^{\circ} \mathrm{C}$ & $\begin{array}{l}\mathrm{R}_{1} \text { line }=0.10 \\
\text { A. } \mathrm{U} . /^{\circ} \mathrm{C} \\
\mathrm{R}_{2} \text { line }=0.33 \\
\text { A. } \mathrm{U} /{ }^{\circ} \mathrm{C}\end{array}$ & 0.21 A.U. $/{ }^{\circ} \mathrm{C}$ \\
\hline $\mathrm{FWHM} /{ }^{\circ} \mathrm{C}$ & $\begin{array}{l}\mathrm{R}_{1} \text { line }=0.005 \\
\mathrm{~nm} /{ }^{\circ} \mathrm{C} \\
\mathrm{R}_{2} \text { line }=0.003 \\
\mathrm{~nm} /{ }^{\circ} \mathrm{C}\end{array}$ & $0.07 \mathrm{~nm} /{ }^{\circ} \mathrm{C}$ \\
\hline
\end{tabular}




\section{Chapter 8 \\ CONCLUSIONS AND FUTURE WORK}

"The horizon leans forward, offering you space to place new steps of change."

Maya Angelou

Many accomplishments were achieved in this work. An experimental setup for fluorescence thermometry on quantum dot thin films was designed and built. The setup consisted of a custom-built sample mount with a specialized heater and surround. The mount was incorporated into an optical imaging and photoluminescence signal collection system which included collection optics, a monochromator, and a data acquisition system. CdSe quantum dots in PMMA have been the archetypal system for studies on quantum confinement with all but a few studies being carried out above cryogenic temperatures. The results published in this dissertation are the first in which the optical properties are quantified for $\mathrm{CdSe} / \mathrm{ZnS}$ QDs in the higher temperature regime of PMMA. A solution processing technique was established for fabricating high-temperature resistant quantum dot sol-gel nanocomposite films. In a separate study, uniformly dispersed highly luminescent $\mathrm{CdSe} / \mathrm{ZnS}$ quantum dots were prepared in a modified $\mathrm{SiO}_{2}$ solution and thin films were fabricated. Absorption spectroscopy was used to investigate the temperature dependent optical absorption properties. Temperature dependent photoluminescence emission was measured and the spectra were characterized and compared against theory. The $\mathrm{SiO}_{2}$ matrix accommodated the $\mathrm{CdSe} / \mathrm{ZnS}$ QDs well 
giving rise to strong excitonic absorption and emission. The data enables establishment of QD thin films that can withstand high temperatures which is critical to quantum dotbased device applications. The nanoscience of the behavior of $\mathrm{CdSe} / \mathrm{ZnS}$ quantum dots under thermal extremes from 295 - $600 \mathrm{~K}$ was studied. The results were compared against rare earth and transition metal ion-doped bulk crystalline materials which are the state-of-the-art in luminescence base thermometry. Specifically, a comprehensive optical spectroscopic study was carried out on bulk crystal alexandrite, $\mathrm{BeAl}_{2} \mathrm{O}_{4}: \mathrm{Cr}^{3+}$. Temperature-dependent luminescent emission from $\mathrm{BeAl}_{2} \mathrm{O}_{4}: \mathrm{Cr}^{3+} \mathrm{R}$ lines were measured and served as a bench mark reference for research on quantum dots. It was found that the QD optical absorption cross section is two times higher than bulk crystalline materials and remains high at thermal extremes. The absorption cross sections for the $\mathrm{CdSe} / \mathrm{ZnS}: \mathrm{SiO}_{2}$ thin films are in general agreement with what has been published in the field to date which has been limited to quantum dots in solution. It was found that the band gap energy shows a linear response with temperature up to $580 \mathrm{~K}$. The Varshni relation is applied to the CdSe/ZnS:PMMA nanocomposite and the $\mathrm{CdSe} / \mathrm{ZnS}: \mathrm{SiO}_{2}$ nanocomposite. The results show that the Varshni relation fit the data up to $580 \mathrm{~K}$ well. The variation in the energy band gap with increasing temperature is more sensitive in QDs than bulk which is a desired characteristic for thermal sensing. The data extends the thermal sensing limit of $\mathrm{CdSe} / \mathrm{ZnS}: \mathrm{SiO}_{2}$ QD thin films and is leveraged toward a luminescence-based thermal sensor.

The CdSe/ZnS: $\mathrm{SiO}_{2}$ quantum dot nanocomposite was implemented as the active element for a first generation thermal sensor. The luminescence-based high temperature fiber optic sensor was designed, fabricated, and tested. The sensors were shown to be 
sensitive over the 295-625 K temperature range. This sensor has potential for aerospace and other applications. In partnership with NASA, specialty fiber optic sensors were designed, fabricated, and tested in a simulated high-temperature atmospheric reentry environment. The sensors were shown to be sensitive over the 295-900 K temperature range for in-flight sensor applications. The sensors maintained structural integrity during 60 seconds. Ionizing species were identified from fluorescence measurements using the optical instrumentation coupled to the sensors. The gaseous species in the flow were identified. The research was a milestone, first-time project in the history of NASA.

Many firsts were accomplished and new experimental processes for QD manipulation came out of this thesis research. As a result, several directions for future work can be cited. A few directions for future research are discussed below.

Photoluminescence decreases with increasing temperature. The PL intensity decreases to low level values above $550 \mathrm{~K}$ making it difficult to measure changes in temperature. This is one limitation that needs to be overcome. High temperature luminescence thermometry encompassed many material processes that can change under thermal extremes. As bulk semiconductors are subjected to heat, thermally induced lattice expansion causes a change in optical and electronic properties. Increased electronphonon coupling is the dominate contribution to the energy band gap shift with increasing temperature in bulk semiconductors. In QDs, lattice spacing changes due to thermal expansion. This change causes a shift in the band gap. Above $580 \mathrm{~K}$, the $\mathrm{CdSe} / \mathrm{ZnS}: \mathrm{SiO}_{2}$ fluorescence rapidly quenches. The PL emission was weak yet, measureable above $580 \mathrm{~K}$. The luminescence properties of CdSe/ZnS core-shell quantum dots can be affected by host materials, film thickness, particle size, and shape. Since the 
$\mathrm{SiO}_{2}$ host matrix can technically withstand much higher temperature $\left(\mathrm{T}_{\mathrm{m}}=1500 \mathrm{~K}\right)$, future work should investigate the effect of QD film thickness on the PL emission at temperatures above $580 \mathrm{~K}$.

It is equally important to investigate the effect of the QD size and shape on the temperature-dependent luminescence properties of the $\mathrm{CdSe} / \mathrm{ZnS}$ quantum dots. In this work, the $\mathrm{CdSe} / \mathrm{ZnS}$ quantum dot particle size calculations and measurements accounted for the $\mathrm{ZnS}$ shell. $\mathrm{ZnS}$ acts as a passivation layer to enhance PL from CdSe. Further analysis of the role of the shell may be useful for high accuracy device modeling.

Over the past few years there has been some work on using nanowires (NWs) for various device applications. Unlike quantum dots, nanowires are asymmetric and should in principle facilitate band transport of photogenerated carriers along the NW length. As a consequence, the mobility of charge carries will be larger than in quantum dots. Nanowires are intrinsically better absorbers than colloidal QDs. Hence, a potential order of magnitude or higher enhancement in the absorption cross sections may be realized which can be used for temperature characterization measurements via absorption spectroscopy. In conventional luminescence thermometry, the issue is not the dopant but the dopant-matrix interaction. If the rare earth (RE) ion or transition metal (TM) ion can marry with the quantum dot then, powerful new nanostructures may be realized for luminescence thermometry. A few studies have recently emerged on the basic science towards RE- and TM ions doped in various low dimensional nanostructures. In an ideal system, the quantum dot emission and the emission from thermally coupled dopant ions would combine to yield a self-referenced temperature sensor. A QD:RE:TM nanocomposite could also support dual-parameter sensing in which the PL emission 
from the $\mathrm{QD}$ is used to measure temperature while the RE or TM is used to measure gases or chemical species.

This dissertation research accomplished a proof-of-concept demonstration of a high temperature luminescence-based QD sensor. For fiber optic thermal sensing devices the numbers published in this dissertation advance the current literature and have provided good accuracy for device framework as demonstrated with $\mathrm{CdSe} / \mathrm{ZnS}: \mathrm{SiO}_{2}$ thermal sensor. The demonstration was achieved after dip-coating $\mathrm{CdSe} / \mathrm{ZnS}: \mathrm{SiO}_{2}$ nanocomposite onto the end tip of an optical fiber. After numerous attempts, a usable coating was cast at the end tip of the fiber. However, alternative QD coated fiber methods such as capillary diffusion can be explored as a way to further enhance the PL quantum yield. Further work will enable the sensor to be used for a specific thermal sensing application. For aerospace applications, testing the fiber stability under vacuum and under various gas flows such as that tested in the HyMETS experiments (see Appendix A) will give stability and sensitivity data needed for a real-time sensor. Miniaturized optics coupled to optical fiber end tip will support a single input/output device. Rare earth oxides have been used for thermometry above $1100 \mathrm{~K}$. Fabricating rare earth oxides at quantum dimensions may enable the development of a new class of quantum confined nanoparticles. These nanoparticles could extend the temperature sensing capability of a QD-based thermal sensor. Advanced sensor applications that can come out from the thermal sensor data include a strain sensor and a multi-sensing QD stress, strain, temperature sensor. 


\section{REFERENCES}

[1] R. P. Feynman, "There's plenty of room at the bottom " J. Microelectromech. Syst., vol. 1, pp. 60-66, 1960.

[2] N. Taniguchi, "On the basic concept of nanotechnology," Proc. Intl Conf. Prof. Eng. Tokyo Part II, 1974.

[3] K. Krishen, "New technology innovations with potential for space applications," Acta Astronautica, vol. 63, pp. 324-333, 2008.

[4] E. Magison, "Physical principles underlie the four common methods," Intech, vol. 48, pp. 39-41, 2001.

[5] P. Nuebert, "Device for indicating the temperature distribution of hot bodies," $U S$ Patent No. 2-071-471, 1937.

[6] L. C. Bradley, "A temperature sensitive phosphor used to measure surface temperatures in aerodynamics," Rev. Sci. Instrum., vol. 24, pp. 219-220, 1953.

[7] P. Czysz and W. P. Dixon, "Quantitative heat transfer measurement using thermographic phosphors," SPIE, vol. 7, pp. 77-79, 1969.

[8] A. Khalid and K. Kontis, "Thermographic phosphors for high temperature measurements: Principles, current state of the art and recent applications," Sensors, vol. 8, pp. 5673-5744, 2008.

[9] T. Katsumata, N. Kawaguchi, Y. Fujimaki, Y. Kiyokawa, H. Aizawa, S. Komuro, T. Morikawa, and E. Toba, "Rare-earth aluminate crystals for fluorescence thermometer application," SICE 2004 Annual Conference, vol. 3, pp. 2512-2515, 2004.

[10] H. Peng, H. Song, B. Chen, J. Wang, S. Lu, X. Kong, and J. Zhang, "Temperature dependence of luminescent spectra and dynamics in nanocrystalline $\mathrm{Y}_{2} \mathrm{O}_{3}: \mathrm{Eu}^{3}, " J$. Phys. Chem. , vol. 118, pp. 3277-3282, 2003.

[11] S. W. Allison and G. T. Gillies, "Remote thermometry with thermographic phosphors: Instrumentation and applications," Rev. Sci. Instrum., vol. 68, pp. 2615-2650, 1997.

[12] H. Uchiyama, H. Aizawa, T. Katsumata, S. Komuro, T. Morikawa, and E. Toba, "Fiber-optic thermometer using Cr-doped $\mathrm{YAlO}_{3}$ sensor head," Rev. Sci. Instrum., vol. 74, pp. 3883-3885, 2003.

[13] H. C. Seat and J. H. Sharp, "Dedicated temperature sensing with C-axis oriented single crystal ruby $\left(\mathrm{Cr}^{3+} \mathrm{Al}_{2} \mathrm{O}_{3}\right)$ fibers: Temperature and strain dependences of $\mathrm{R}$ line fluorescence," IEEE Trans. Instrum. Meas., vol. 53, pp. 140-154, 2004. 
[14] L. H. Ye, J. F. Zhang, and Y. Shi, "Growth and characteristics of $\mathrm{Cr}^{3+}$ : YAG crystal fiber for fluorescence decay temperature sensor," Rev. Sci. Instrum., vol. $77,2006$.

[15] B. Dong, T. Yang, and M. K. Lei, "Optical high temperature sensor based on green up-conversion emissions in $\mathrm{Er}^{3+}$ doped $\mathrm{Al}_{2} \mathrm{O}_{3}$," Sen. Actuators, B vol. 123, pp. 667-670, 2007.

[16] B. Dong, X. J. Wang, C. R. Li, and D. P. Liu, "Er ${ }^{3+}-\mathrm{Y}^{3+}$-codoped $\mathrm{Al}_{2} \mathrm{O}_{3}$ for hightemperature sensing," IEEE Photonics Technol. Lett., vol. 20, pp. 117-119, 2008.

[17] V. K. Rai and C. B. de Araujo, "Fluorescence intensity ratio technique for $\mathrm{Sm}^{3+}$ doped calibo glass," Spectrochim. Acta, Part A-, vol. 69, pp. 509-512, 2008.

[18] H. Xiao, J. D. Deng, G. Pickrell, R. G. May, and A. B. Wang, "Single-crystal sapphire fiber-based strain sensor for high-temperature applications," $J$.

Lightwave Technol., vol. 21, pp. 2276-2283, 2003.

[19] F. Sidiroglou, S. A. Wade, N. M. Dragomir, G. W. Baxter, and S. F. Collins, "Effects of high-temperature heat treatment on $\mathrm{Nd}^{3+}$-doped optical fibers for use in fluorescence intensity ratio based temperature sensing," Rev. Sci. Instrum., vol. 74, pp. 3524-3530, 2003.

[20] H. Aizawa, H. Uchiyama, T. Katsumata, S. Komuro, T. Morikawa, H. Ishizawa, and E. Toba, "Fibre-optic thermometer using sensor materials with long fluorescence lifetime," Meas. Sci. Technol., vol. 15, pp. 1484-1489, 2004.

[21] M. A. M. Gamez, S. G. C. Vicente, A. V. Kir'yanov, and A. Martinez-Rios, "Effect of erbium-ytterbium on the sensitivity of sensors of temperature based on fibre optic preform Er-Yb: $\mathrm{BaF}_{2}-\mathrm{P}_{2} \mathrm{O}_{5}, "$ Meas. Sci. Technol., vol. 18, pp. 32533256, 2007.

[22] G. Schmid, "Nanopaticles: From Theory to Application," John Wiley \& Sons, 1991.

[23] Y. H. Wu, K. Arai, and T. Yao, "Temperature dependence of the photoluminescence of $\mathrm{ZnSe} / \mathrm{ZnS}$ quantum-dot structures," Phys. Rev. B., vol. 53, pp. 10485-10488, 1996.

[24] G. W. Walker, V. C. Sundar, C. M. Rudzinski, A. W. Wun, M. G. Bawendi, and D. G. Nocera, "Quantum-dot optical temperature probes," Appl. Phys. Lett., vol. 83, pp. 3555-3557, 2003.

[25] O. Labeau, P. Tamarat, and B. Lounis, "Temperature dependence of the luminescence lifetime of single CdSe/ZnS quantum dots," Phys. Rev. Lett., vol. 90, p. 257404, 2003. 
[26] V. Biju, Y. Makita, A. Sonoda, H. Yokoyama, Y. Baba, and M. Ishikawa, "Temperature-sensitive photoluminescence of CdSe quantum dot clusters," $J$. Phys. Chem. B., vol. 109, pp. 13899-13905, 2005.

[27] H. Song and S. Lee, "Photoluminescent (CdSe) ZnS quantum dotpolymethylmethacrylate polymer composite thin films in the visible spectral range," Nanotechnol., vol. 18, p. 055402, 2007.

[28] H. C. Y. Yu, S. G. Leon-Saval, A. Argyros, and G. W. Barton, "Temperature effects on emission of quantum dots embedded in polymethylmethacrylate," Appl. Opt., vol. 49, pp. 2749-2752, 2010.

[29] S. Li, Zhang, K., Yang, J. M.,Lin, L. W., Yang, H., "Single quantum dots as local temperature markers," Nano Lett., vol. 7, pp. 3102-3105, 2007.

[30] A. M. Smith and S. M. Nie, "Semiconductor nanocrystals: structure, properties, and band gap engineering," Acc. Chem. Res., vol. 43, pp. 190-200, 2010.

[31] Y. Wang and N. Herron, "Nanometer-sized semiconductor clusters - materials, synthesis, quantum size effects, and photophysical properties," J. Phys. Chem., vol. 95, pp. 525-532, 1991.

[32] A. L. Efros and M. Rosen, "The electronic structure of semiconductor nanocrystals," Annu. Rev. Mater. Sci., vol. 30, pp. 475-521, 2000.

[33] P. Daukantas, "Light in flight: Optical applications in civilian aviation," Optics and Photonics News, vol. 22, pp. 40-45, 2011.

[34] "High-temperature sensing with quantum dots," in www.nanotechweb.org, ed, 2011.

[35] G. Konstantatos and E. H. Sargent, "Nanostructured materials for photon detection," Nat. Nanotechnol., vol. 5, pp. 391-400, 2010.

[36] M. Faraday, "Experimental relations of gold (and other metals) to light " Philos. Trans. R Soc. London, Ser. A vol. 147, pp. 145-181, 1857.

[37] A. I. Ekimov and A. A. Onushchenko, "Quantum Size Effect in the OpticalSpectra of Semiconductor Micro-Crystals," Solid State Commun., vol. 16, pp. 775-778, 1982.

[38] A. I. Ekimov, A. L. Efros, and A. A. Onushchenko, "Quantum size effects in semiconductor microcrystals," Solid State Commun., vol. 56, pp. 921-924, 1985.

[39] R. Rossetti, S. Nakahara, and L. Brus, "Quantum size effects in the redox potentials, resonance Raman spectra, and electronic spectra of CdS crystallites in aqueous solution," J. Chem. Phys., vol. 79, pp. 1086-1087, 1983. 
[40] L. E. Brus, "A simple model for the ionization potential, electron affinity, and aqueous redox potentials of small semiconductor crystallites," J. Chem. Phys., vol. 79, pp. 5566-5571, 1983.

[41] A. L. Efros, "Interband absorption of light in a semiconductor sphere," S. Phys. Semicond., vol. 16, pp. 772-775, 1982.

[42] C. B. Murray and M. G. Bawendi, "Synthesis and physical characterization of IIVI semiconductor nanocrystallites (Quantum Dots)," J. Am. Chem. Soc., vol. 205, pp. 136-140, 1993.

[43] http://en.wikipedia.org/wiki/File:Wurtzite_polyhedra.png.

[44] B. Cordero, V. Gomez, A. E. Platero-Prats, M. Reves, J. Echeverria, E. Cremades, F. Barragan, and S. Alvarez, "Covalent radii revisited," Dalton Trans., pp. 28322838, 2008.

[45] http://smu.edu/catco/research/nanotech34.html.

[46] V. I. Klimov, "Spectral and dynamical properties of multilexcitons in semiconductor nanocrystals," Annu. Rev. Phys. Chem., vol. 58, pp. 635-673, 2007.

[47] C. B. Murray, C. R. Kagan, and M. G. Bawendi, "Synthesis and characterization of monodisperse nanocrystals and close-packed nanocrystal assemblies," Annual Review of Materials Science, vol. 30, pp. 545-610, 2000.

[48] http://ookaboo.com/o/pictures/picture/13447035.

[49] H. Mao, J. Chen, J. Wang, Z. Li, N. Dai, and Z. Zhu, "Photoluminescence investigation of CdSe quantum dots and the surface state effect," Physica E, vol. 27, pp. 124-128, 2005.

[50] L. E. Brus, "Electron-electron and electron-hole interactions in small semiconductor crystallites: The size dependence of the lowest excited electronic state," J. Chem. Phys., vol. 80, pp. 4403-4409, 1984.

[51] J.-B. Xia, "Electronic structures of zero-dimensional quantum wells," Phys. Rev. B, vol. 40, pp. 8500-8507, 1989.

[52] D. I. Chepic, A. L. Efros, A. I. Ekimov, M. G. Ivanov, V. A. Kharchenko, I. A. Kudriavtsev, and T. V. Yazeva, "Auger ionization of semiconductor quantum drops in a glass matrix," J. Lumin., vol. 47, pp. 113-127, 1990.

[53] L. Brus, "Electronic wave-functions in semiconductor clusters - experiment and theory," J. Phys. Chem., vol. 90, pp. 2555-2560, 1986. 
[54] C. J. Murphy and J. L. Coffer, "Quantum dots: A primer," Appl. Spectrosc., vol. 56, pp. 16a-27a, 2002.

[55] M. V. R. Krishna and R. A. Friesner, "Quantum confinement effects in semiconductor clusters," J. Chem. Phys., vol. 95, pp. 8309-8322, 1991.

[56] A. Franceschetti, A. Williamson, and A. Zunger, "Addition spectra of quantum dots: The role of dielectric mismatch," J. Phys. Chem. B, vol. 104, pp. 3398-3401, 2000.

[57] M. A. Hines and P. Guyot-Sionnest, "Synthesis and characterization of strongly luminescing ZnS-capped CdSe nanocrystals," J. Phys.Chem., vol. 100, pp. 468$471,1996$.

[58] D. V. Talapin, A. L. Rogach, A. Kornowski, M. Haase, and H. Weller, "Highly luminescent monodisperse $\mathrm{CdSe}$ and $\mathrm{CdSe} / \mathrm{ZnS}$ nanocrystals synthesized in a hexadecylamine-trioctylphosphine oxide-trioctylphospine mixture," Nano Lett., vol. 1, pp. 207-211, 2001.

[59] I. Mekis, D. V. Talapin, A. Kornowski, M. Haase, and H. Weller, "One-pot synthesis of highly luminescent $\mathrm{CdSe} / \mathrm{CdS}$ core-shell nanocrystals via organometallic and "greener" chemical approaches," J. Phys.Chem. B., vol. 107, pp. 7454-7462, 2003.

[60] P. M. Jorge, M. A. Trindade, T. Santos, J. L. Farahi, F., "Optical fiber sensing using quantum dots," Sensors vol. 7, pp. 3489-3534, 2007.

[61] B. O. Dabbousi, J. RodriguezViejo, F. V. Mikulec, J. R. Heine, H. Mattoussi, R. Ober, K. F. Jensen, and M. G. Bawendi, "(CdSe)ZnS core-shell quantum dots: Synthesis and characterization of a size series of highly luminescent nanocrystallites," J. Phys. Chem. B, vol. 101, pp. 9463-9475, 1997.

[62] B. T. Suo, X. Su, J. Wu, D. Chen, A. Wang, and Z. H. Guo, "Poly (vinyl alcohol) thin film filled with CdSe-ZnS quantum dots: Fabrication, characterization, and optical properties," Mater. Chem. and Phys., vol. 119, pp. 237-242, 2010.

[63] T. V. Torchynska, J. Douda, and R. P. Sierra, "Photoluminescence of core-shell CdSe/ZnS quantum dots of different sizes," Phys. Status Solidi C, vol. 6, pp. S143-S145, 2009.

[64] Z. Zhang, J. C. Li, and Q. Jiang, "Modelling for size-dependent and dimensiondependent melting of nanocrystals," J. Phys. D: Applied Physics, vol. 33, pp. 2653-2656, 2000.

[65] M. F. Ashby, Ferriera Paulo J., Schodeck, Daniel L., Nanomaterials, Nanotechnologies, and Design. Burlington: Elsievier, 2009. 
[66] K. K. Nanda, S. N. Sahu, and S. N. Behera, "Liquid-drop model for the sizedependent melting of low-dimensional systems," Phys. Rev. A, vol. 66, p. 013208, 2002.

[67] D. Ricard, M. Ghanassi, and M. C. Schanneklein, "Dielectric confinement and the linear and non-linear optical properties of semiconductor-doped glasses," Opt. Commun., vol. 108, pp. 311-318, 1994.

[68] P. R. Yu, M. C. Beard, R. J. Ellingson, S. Ferrere, C. Curtis, J. Drexler, F. Luiszer, and A. J. Nozik, "Absorption cross-section and related optical properties of colloidal InAs quantum dots," J. Phys. Chem. B., vol. 109, pp. 7084-7087, 2005 .

[69] Y.P. Varshni, "Temperature dependence of the energy gap in semiconductors," Physica, vol. 34, pp. 149-154, 1967.

[70] D. Valerini, A. Creti, M. Lomascolo, L. Manna, R. Cingolani, and M. Anni, "Temperature dependence of the photoluminescence properties of colloidal $\mathrm{CdSe} / \mathrm{ZnS}$ core/shell quantum dots embedded in a polystyrene matrix," Phys. Rev. $B$, vol. 71, p. 235409, 2005.

[71] A. Joshi, K. Y. Narsingi, M. O. Manasreh, E. A. Davis, and B. D. Weaver, "Temperature dependence of the band gap of colloidal CdSe/ZnS core/shell nanocrystals embedded into an ultraviolet curable resin," Appl. Phys. Lett., vol. 89, pp. 1319071-3, 2006.

[72] S. Rudin, T. L. Reinecke, and B. Segall, "Temperature-dependent exciton linewidths in semiconductors," Phys. Rev. B, vol. 42, pp. 11218-11231, 1990.

[73] F. Gindele, K. Hild, W. Langbein, and U. Woggon, "Temperature-dependent line widths of single excitons and biexcitons," J. Lumin., vol. 87-9, pp. 381-383, 2000.

[74] P. T. Jing, J. J. Zheng, M. Ikezawa, X. Y. Liu, S. Z. Lv, X. G. Kong, J. L. Zhao, and Y. Masumoto, "Temperature-dependent photoluminescence of CdSe-Core CdS/CdZnS/ZnS-multishell quantum dots," J. Phys. Chem. C, vol. 113, pp. 13545-13550, 2009.

[75] R. D. Deegan, O. Bakajin, T. F. Dupont, G. Huber, S. R. Nagel, and T. A. Witten, "Capillary flow as the cause of ring stains from dried liquid drops," Nature, vol. 389, pp. 827-829, 1997.

[76] R. D. Deegan, O. Bakajin, T. F. Dupont, G. Huber, S. R. Nagel, and T. A. Witten, "Contact line deposits in an evaporating drop," Phys. Rev. E, vol. 62, pp. 756-765, 2000.

[77] J. C. Loudet, A. G. Yodh, and B. Pouligny, "Wetting and contact lines of micrometer-sized ellipsoids," Phys. Rev. Lett., vol. 97, pp. 018304-1-018304-4, 2006. 
[78] S. Maenosono, C. D. Dushkin, S. Saita, and Y. Yamaguchi, "Growth of a semiconductor nanoparticle ring during the drying of a suspension droplet," Langmuir, vol. 15, pp. 957-965, 1999.

[79] P. J. Yunker, T. Still, M. A. Lohr, and A. G. Yodh, "Suppression of the coffeering effect by shape-dependent capillary interactions," Nature, vol. 476, pp. 308$311,2011$.

[80] I. Horcas, R. Fernandez, J. M. Gomez-Rodriguez, J. Colchero, J. Gomez-Herrero, and A. M. Baro, "WSxM: A software for scanning probe microscopy and a tool for nanotechnology," Rev. Sci. Instrum., vol. 78, p. 0137051, 2007.

[81] "Lambda 800-900 Users Guide."

[82] V. Svorcík, J. Králová, V. Rybka, J. PleSek, J. Cervená, and V. Hnatowicz, Temperature dependence of the permittivity of polymer composites vol. 39: John Wiley \& Sons, Inc., 2001.

[83] M. S. Gaponenko, A. A. Lutich, N. A. Tolstik, A. A. Onushchenko, A. M. Malyarevich, E. P. Petrov, and K. V. Yumashev, "Temperature-dependent photoluminescence of $\mathrm{PbS}$ quantum dots in glass: Evidence of exciton state splitting and carrier trapping," Phys. Rev. B, vol. 82, p. 125320, 2010.

[84] A. Al Salman, A. Tortschanoff, M. B. Mohamed, D. Tonti, F. van Mourik, and M. Chergui, "Temperature effects on the spectral properties of colloidal CdSe nanodots, nanorods, and tetrapods," Appl. Phys. Lett., vol. 90, pp. 093104-1093104-3, 2007.

[85] D. Pugh-Thomas, B. M. Walsh, and M. C. Gupta, "Quantum dot quantum efficiency measurements," Under preparation, 2012.

[86] E. D. Palik, "Handbook of optical constants of solids," ed: Elsevier, 1983.

[87] R. C. Hilborn, "Einstein coefficients, cross-sections, f values, dipole moments, and all that," Am. J. Phys., vol. 50, pp. 982-986, 1982.

[88] A. Striolo, J. Ward, J. M. Prausnitz, W. J. Parak, D. Zanchet, D. Gerion, D. Milliron, and A. P. Alivisatos, "Molecular weight, osmotic second virial coefficient, and extinction coefficient of colloidal CdSe nanocrystals," J. Phys. Chem. B, vol. 106, pp. 5500-5505, 2002.

[89] "PeakFit Graphical Analysis Software v.4.12."

[90] C. D. Donega, "Formation of nanoscale spatially indirect excitons: Evolution of the type-II optical character of CdTe/CdSe heteronanocrystals," Phys. Rev. B, vol. 81, pp. 165303-1-165303-20, 2010. 
[91] A. Franceschetti and A. Zunger, "Optical transitions in charged CdSe quantum dots," Phys. Rev. B, vol. 62, pp. R16287-R16290, 2000.

[92] V. I. Klimov, S. A. Ivanov, J. Nanda, M. Achermann, I. Bezel, J. A. McGuire, and A. Piryatinski, "Single-exciton optical gain in semiconductor nanocrystals," Nature, vol. 447, pp. 441-446, 2007.

[93] R. S. Singh, S. Bhushan, and A. K. Singh, "Studies on nano-crystalline properties of chemically deposited CdSe films," Chalcogenide Lett., vol. 7, pp. 375-381, 2010 .

[94] C. Cheng and W. Xiaoyan, "A comparative study of spectral characteristics of $\mathrm{CdSe}$ and CdSe/ZnS quantum dots," in Int. Symp. on Biophotonics, Nanophotonics, and Metamaterials 2006 Hangzhou, China.

[95] C. A. Leatherdale, W. K. Woo, F. V. Mikulec, and M. G. Bawendi, "On the absorption cross section of CdSe nanocrystal quantum dots," J. Phys. Chem. B., vol. 106, pp. 7619-7622, 2002.

[96] C. D. Donega and R. Koole, "Size dependence of the spontaneous emission rate and absorption cross section of CdSe and CdTe quantum dots," J. Phys. Chem. C, vol. 113, pp. 6511-6520, 2009.

[97] J. Jasieniak, L. Smith, J. van Embden, P. Mulvaney, and M. Califano, "Reexamination of the size-dependent absorption properties of CdSe quantum dots," J. Phys. Chem. C., vol. 113, pp. 19468-19474, 2009.

[98] J. McBride, J. Treadway, L. C. Feldman, S. J. Pennycook, and S. J. Rosenthal, "Structural basis for near unity quantum yield core/shell nanostructures," Nano Lett., vol. 6, pp. 1496-1501, 2006.

[99] D. Pugh-Thomas, B. M. Walsh, and M. C. Gupta, "CdSe(ZnS) nanocomposite luminescent high temperature sensor," Nanotechnol., vol. 22, p. 185503, 2011.

[100] N. M. Ravindra and V. K. Srivastava, "Temperature dependence of the energy gap in semiconductors," J. Phys. Chem. Solids, vol. 40, pp. 791-793, 1979.

[101] V. Veilleux, D. Lachance-Quirion, K. Dore, D. B. Landry, P. G. Charette, and C. N. Allen, "Strain-induced effects in colloidal quantum dots: lifetime measurements and blinking statistics," Nanotechnol., vol. 21, p. 134024, 2010.

[102] S. H. Park and Y. H. Cho, "Strain and piezoelectric potential effects on optical properties in $\mathrm{CdSe} / \mathrm{CdS}$ core/shell quantum dots," J. Appl. Phys., vol. 109, p. $113103,2011$.

[103] A. V. Baranov, Y. P. Rakovich, J. F. Donegan, T. S. Perova, R. A. Moore, D. V. Talapin, A. L. Rogach, Y. Masumoto, and I. Nabiev, "Effect of ZnS shell 
thickness on the phonon spectra in CdSe quantum dots," Phys. Rev. B, vol. 68, p. 165306, 2003.

[104] H. Lange, M. Artemyev, U. Woggon, T. Niermann, and C. Thomsen, "Experimental investigation of exciton-LO-phonon couplings in CdSe/ZnS core/shell nanorods," Phys. Rev. B, vol. 77, p. 193303, 2008. 


\section{APPENDIX A \\ Advanced Optical Instrumentation for Exploration Vehicles: Orion Le-X Radiative Heating Project}

\section{Introduction}

Research and development on electro-optical instrumentation for radiation measurements was carried out under the NASA Orion Le-X TPS Radiative Heating Project. The mission of Orion Le-X Radiative Heating Project was to demonstrate the utility of using specialty sensors embedded in an ablative thermal protection system (TPS) for in-flight measurements. The target measurements were bow shock radiation and TPS heating. The study was sought to further minimize risks for controlled reentry into extra-terrestrial atmospheres. The reentry test environments under study in Orion Le-X were the Earth and moon. Using innovative optical instrumentation, a series of emission spectroscopic experiments were carried out in the NASA Hypersonic Materials Environmental Test System (HYMETS). Shock heated air ahead of the entry vehicle's aeroshell was simulated in HYMETS. Chemical species such as $\mathrm{N}_{2} \mathrm{O}_{2}, \mathrm{NO}, \mathrm{NO}^{+}, \mathrm{N}_{2}{ }^{+}, \mathrm{N}$, $\mathrm{O}$, and $\mathrm{e}^{-}$were identified. This series of experiments were the first use of optical fibers within ablative TPS materials.

\section{Background}

Significant advances in photonics in the post-Apollo decades have enabled the development of new technologies and materials for thermal protection systems. Table A1 gives a brief review of select NASA flight programs in which emission spectroscopic experiments were carried out to obtain in-flight flow data. The Flight Investigation of the Reentry Environment (Fire II), Apollo, and the Bow Shock Ultraviolet Experiments (BSUV) have flown and collected data in-situ. Fire II is to date the only flight spectral data at velocities greater than $8 \mathrm{~km} / \mathrm{s}^{1}$. Fire II data was for a non-ablative heat shield. BSUV flights demonstrated that optical fibers obtained good views of the shock layer radiation. The BSUV work also showed the use of fiber optics allowed flexibility in the placement of the detector hardware (monochromator). Fire II and Apollo both incorporated high-temperature resistant windows as the signal collection entry pathway ${ }^{2}$. Two of the major challenges with the window-concept are (1) maintaining structural integrity in extreme environments and (2) remaining transmissive over the spectral range of interest. These challenges extend to present-day flight programs and remain nontrivial. Table A-1 clearly demonstrates the need for new data to help drive TPS development for new crew exploration vehicles and planetary probes. 
Table A-1 Select Flight Program Data

\begin{tabular}{|c||c|l|c|c|c|}
\hline $\begin{array}{c}\text { Flight } \\
\text { Program }\end{array}$ & Year & $\begin{array}{c}\text { Design } \\
\text { Concept }\end{array}$ & $\begin{array}{c}\text { Spectral } \\
\text { Range }\end{array}$ & $\begin{array}{c}\text { Velocity } \\
(\mathrm{km} / \mathrm{s})\end{array}$ & $\begin{array}{c}\text { Altitude } \\
(\mathrm{km})\end{array}$ \\
\hline Fire-II & 1965 & $\begin{array}{l}\text { Radiometer } \\
\text { quartz- } \\
\text { window }\end{array}$ & UV & $>8$ & $40-80$ \\
\hline Apollo 4 & 1967 & $\begin{array}{l}\text { Radiometer } \\
\text { quartz- } \\
\text { window }\end{array}$ & $200 \mathrm{~nm}$ & 10.7 & $80-40$ \\
\hline BSUV & 1990 & $\begin{array}{l}\text { Lens-capped } \\
\text { Fiber-optic } \\
\text { bundles }\end{array}$ & $200-400 \mathrm{~nm}$ & 3.5 & $38-70$ \\
\hline DEBI & 2003 & $\begin{array}{l}\text { Fiber-optics } \\
\text { bundles, } \\
\text { sapphire lens } \\
\text { caps }\end{array}$ & $\begin{array}{l}\text { MWIR and } \\
\text { UV }\end{array}$ & 3.5 & $40-70$ \\
\hline
\end{tabular}

*Dual-Mode Experiment on Bow-Shock Interactions

Planetary probes are used to explore the solar system and study phenomena not observable from Earth. Travel to planets and their moons is characterized by high speeds, large masses, and long distances associated with the major bodies of the solar system. Atmospheric reentry is required as the terminal or intermediate phase of missions. Flight speeds range from $6 \mathrm{~km} / \mathrm{s}$ for Titan entry to $50 \mathrm{~km} / \mathrm{s}$ for direct entry to Jupiter. For vehicles traveling at hypersonic speeds, the thermal protection system (TPS) is a single point-failure system. The TPS shields the vehicle structure and payload. It enables safe deployment of probes, landers, balloons, and other spacecraft. An understanding of the spacecraft operation at these speeds is obtained through characterization of heating, inertial and viscous loads, and dynamic and static control. These measurands can be obtained using instrumented thermal protection systems. 


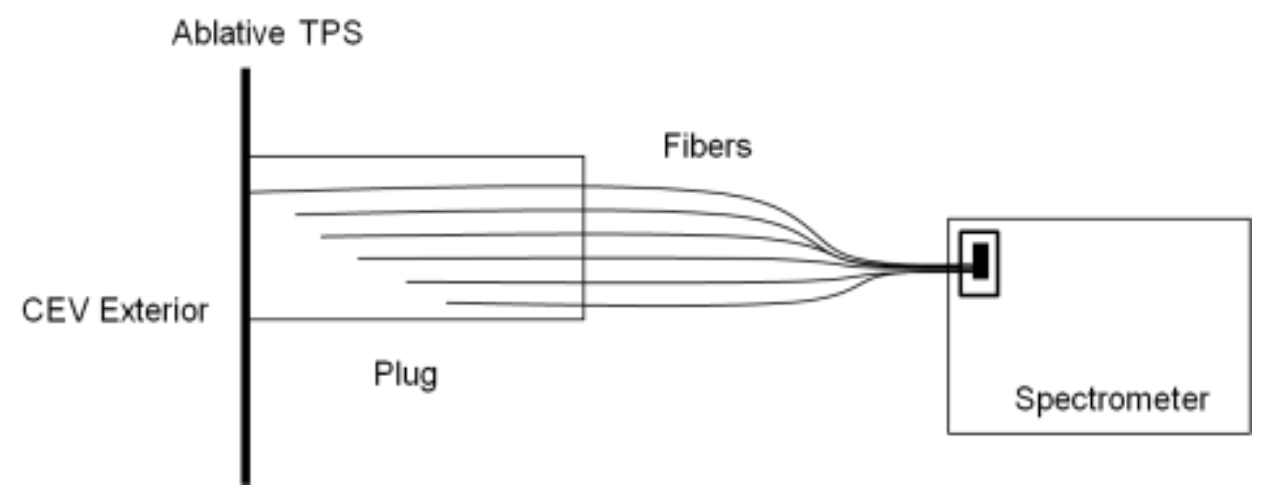

Figure A1. Schematic of step-wise optical flight sensor.

On promising approach to instrumented TPS is the use of optical fibers to transmit collected radiation. The NASA Orion Le-X TPS Radiative Heating Project builds from lessons learned in BSUV and $\mathrm{DEBI}^{3}$. Le-X considers new ground-test strategies. The project proposed the use of optical fibers and compact spectrometers to collect and transmit signal. Miniaturized grating spectrometers coupled to optical fibers will support multiplexing and versatility in measurements. The new design proposed the use of optical fibers embedded into a structural composite thermal protection material. The Apollo 4 design used a thermopile quartz window mounted at the bottom of the well. In the Le-X design the fiber bundle replaces the quartz window and is recessed such that ablative materials is not caught up in the well but will deposit to the side. The schematic of the stepwise fiber-optic assembly is shown in Figure A1. Fiber bundles are embedded in a step-wise manner into a multilayered "plug" of TPS ablator material. As the TPS material ablates, the end-tip of the fiber optic is exposed, burns off or melts. The next fiber then becomes the light conduit into the spectrometer. The small plug enables fibers to be multiplexed at the spectrometer, allowing at low cost, the placement of several of these sensors around the spacecraft.

The Le-X TPS Radiative Heating Project goals were to develop a compact, low intrusive shuttle health monitoring (SHM) sensor for the measurement of parameters, such as temperature at locations not readily accessible. The complete proposal and the preliminary data report are found elsewhere ${ }^{4,5}$. The research objectives carried out in this investigation were (1) test the feasibility of a new fiber optic-based concept for optical access through an aeroshell heat shield for spectroscopic measurements, (2) examine the transmission characteristics of the specially fiber under high enthalpy flow, and (3) determine the most favorable configuration for maximizing the life of embedded optical fibers. In this research, small prototypes were designed, tested, and evaluated in the NASA Langley Hypersonic Materials Environmental Test System (HyMETS) arc jet facility. The arc jet test facility and optical instrumentation used for data collection are 
described below. The test model design and assembly is presented. Results are reported. Then the conclusions from the project and future directions are brought forward.

\section{Arc jet Facility}

The NASA Langley HyMETS is an arc jet test facility used to evaluate the response of materials and coatings to hypersonic air flows. It is primarily used for developing measurement techniques and screening new materials. An arc jet is a device in which gases are heated to very high temperatures and high supersonic and hypersonic speeds by a continuous electrical arc between two sets of electrodes. The gases pass through a nozzle aimed at a test sample under vacuum. The gases flow over the test sample providing an approximation of surface temperature, pressure, and gas enthalpies experienced by a space vehicle during atmospheric reentry. Process gases include nitrogen $\left(\mathrm{N}_{2}\right)$, oxygen $\left(\mathrm{O}_{2}\right)$, carbon dioxide $\left(\mathrm{CO}_{2}\right)$, and argon $(\mathrm{Ar})$. Figure A2 is picture of the HyMETS facility. The gas cylinders and mass flow controllers sit in the rear of the chamber.

A $400 \mathrm{~kW}$ power supply runs the arc jet up to mach 5 . Ar (5\% by mass) is injected at the anode. Two different test gases were used in this research: an $\mathrm{N}_{2}$ and an Earth-Air simulant were mixed and injected into the flow. The gases are heated and expanded to high temperatures and speeds by a continuous electrical arc between two set of electrodes. User specified test gases are sheathed over copper electrodes and are accelerated through a nozzle aimed at the test coupon. The HYMETS supports a small 25.4 ( 1 inch) diameter test coupon. The nozzle is made of copper with a $12.7 \mathrm{~mm}(0.5$ inch) diameter throat and a $63.5 \mathrm{~mm}(2.5 \mathrm{inch})$ diameter exit. The inside of the test chamber and a schematic of the arc plasma generator and test nozzle are shown in Figure A3. A 150 ton cooling tower is used for the arc column. A two-stage set of rotary piston vacuum pumps maintain the pressure. A separate room is equipped with the data acquisition system which allows automated control of the test conditions. Table A-2 is a list of the facility operating parameters ${ }^{6}$. 


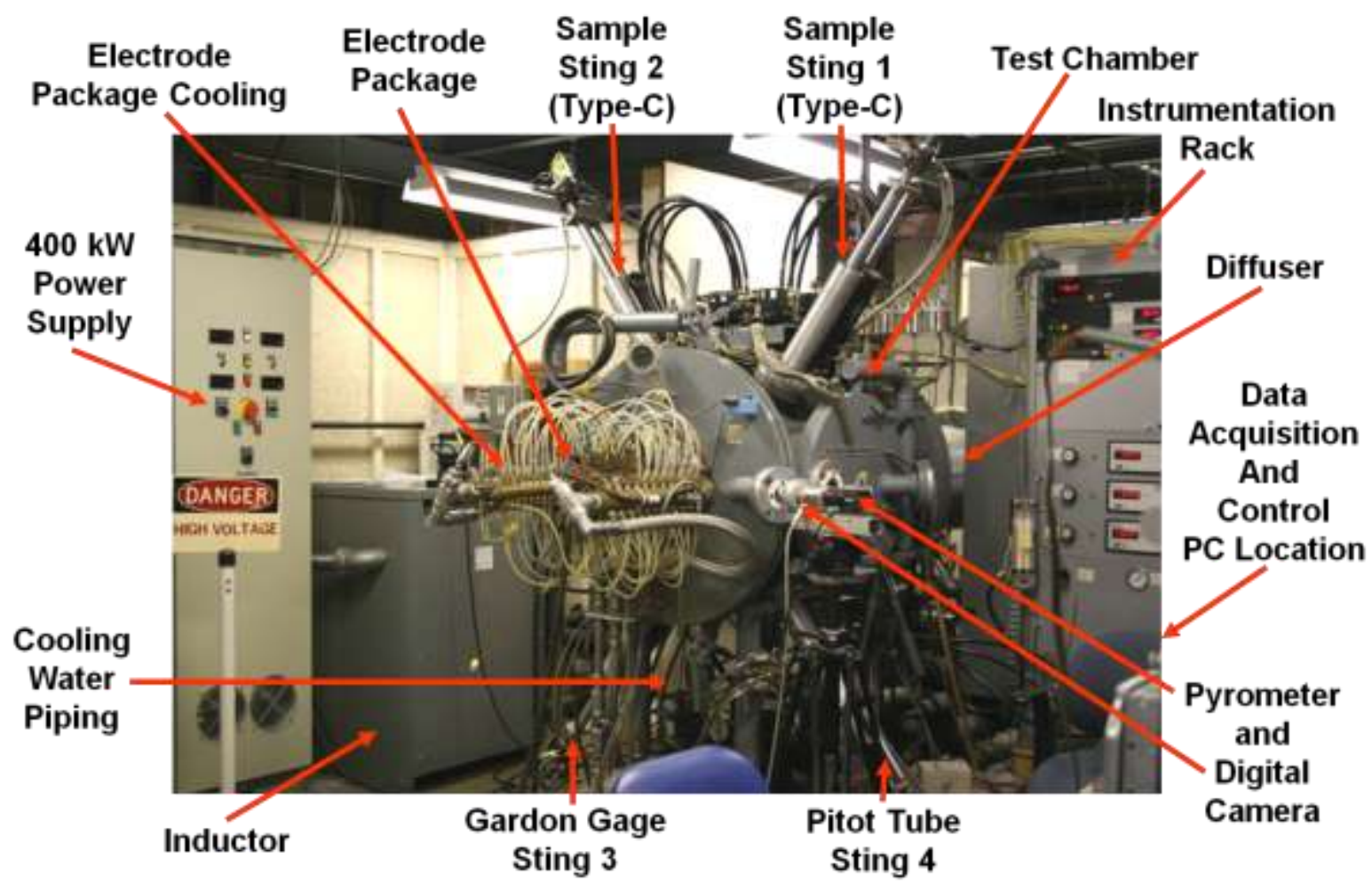

Figure A2. HyMETS Facility. 


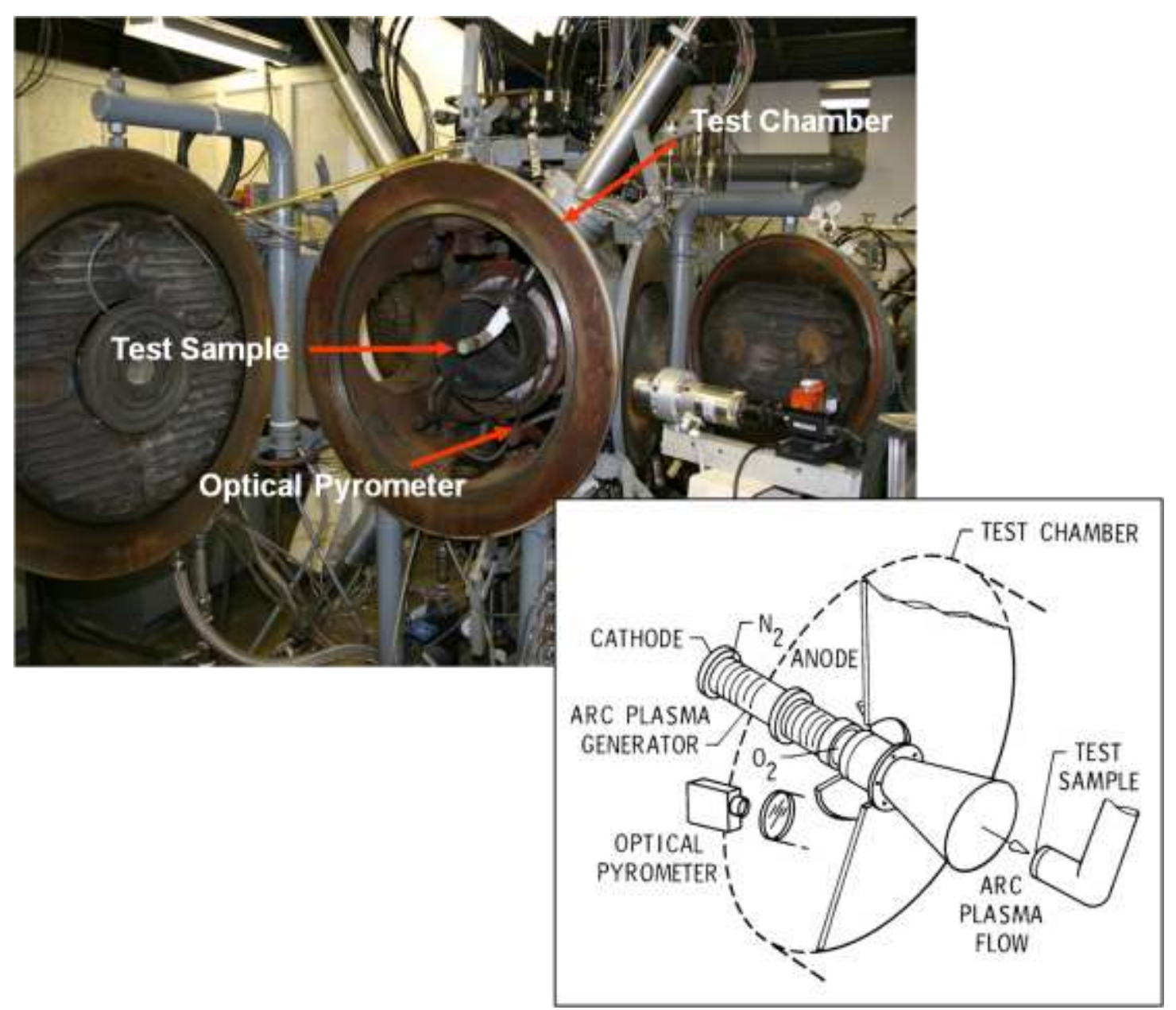

Figure A3. NASA HyMETS test chamber and a schematic of the arc plasma generator and test nozzle. 
Table A-2 HyMETS operating parameters. Data shown in the third column specifies the loads used for the runs taken in this work.

\begin{tabular}{|c|c|}
\hline Parameter & Operating Range \\
\hline \hline Surface Temperature & $1000-3500^{\circ} \mathrm{F}$ \\
\hline Cold Wall Heat Flux & $\sim 166-\sim 736 \mathrm{BTU} / \mathrm{ft}^{2}$-sec \\
\hline Stagnation Pressure & $0.009-0.053 \mathrm{~atm}(7$ to $\sim 40$ Torr) \\
\hline Enthalpy & Bulk: $2400-\sim 11125 \mathrm{BTU} / \mathrm{lb}$ \\
\hline Current & $70-400$ Amps \\
\hline Voltage & $350-800$ Volts \\
\hline Mass Flow Rates & $35-300$ SLPM \\
\hline Arc Pressure & $0.13-1.29$ atm (100- 980 Torr) \\
\hline Chamber Pressure & $1.3 \mathrm{E}-4-2.3 \mathrm{E}-3$ atm (0.1 to 2.0 Torr) \\
\hline
\end{tabular}




\section{Model Design and Optical Fiber Assembly}

Figure A4 is a schematic of a test model. The test models were 2 inches in diameter and 1.5 inches thick. As shown in the figure, the TPS puck was mounted to an insulating tile that was supported by an aluminum backing plate. Alignment dowels were used for stability and to maintain orientation. Two candidate thermal protection system (TPS) materials were investigated: phenolic impregnated carbon ablator (PICA) and phenolic carbon (PhenCarb 28). Extensive testing, modeling, and development have been carried out on PICA and PhenCarb as well as other TPS materials such as Avocoat. PICA is a low density $\left(0.257 \mathrm{~g} / \mathrm{cm}^{3}\right)$ composite ablative tile. It is composed of carbon fibers impregnated with phenolic resin. PICA was used most recently on Stardust and the Mars Science Laboratory. Like PICA, PhenCarb $28\left(\rho=0.45 \mathrm{~g} / \mathrm{cm}^{3}\right)$ also has an extensive NASA flight heritage including Galileo.

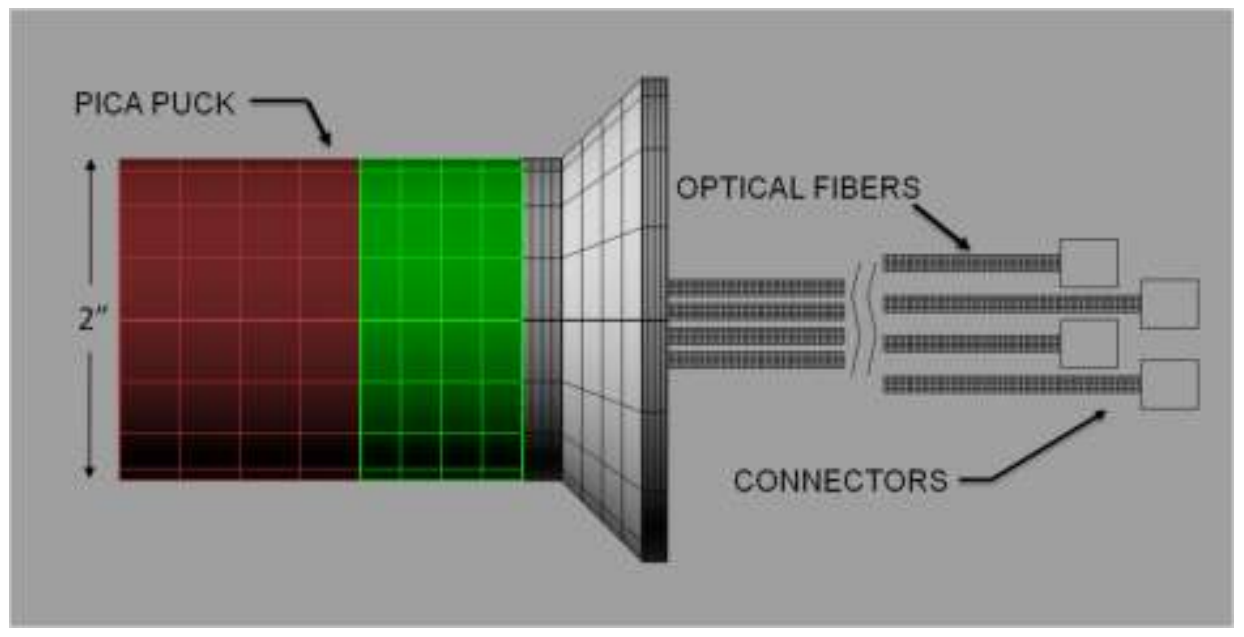

Figure A4. Schematic of test model.

Samples of PICA and PhenCarb 28 used in this research are shown in Figure A5. Figure A6 is a ghost-view of the model showing bare fused silica optical fibers installed via offaxis machined holes. Defined in Figure A7, the fibers were installed $15-20^{\circ}$ from normal away from the model centerline axis. The fused silica fibers had diameters of 300, 400, and $600 \mu \mathrm{m}$. The optical fibers were installed in the models at depths of 0.10,0.20, and 0.30 inches. The fibers were approximately 50 inches in length and had either FC or SMA connectors. The fibers were routed from the test article through Sting Arm 1 (see Figure A2) to the data acquisition system. 

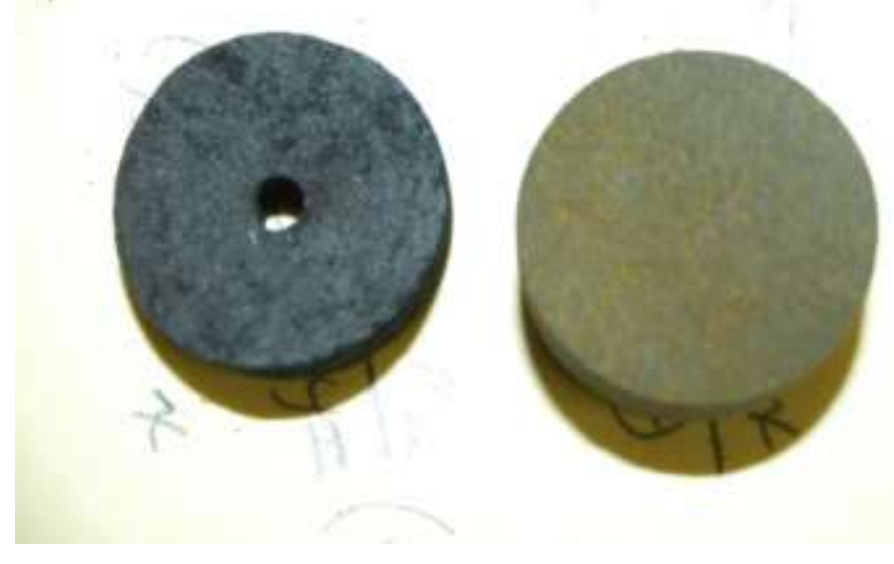

Figure A5. PICA and PhenCarb 28 (black) TPS samples.

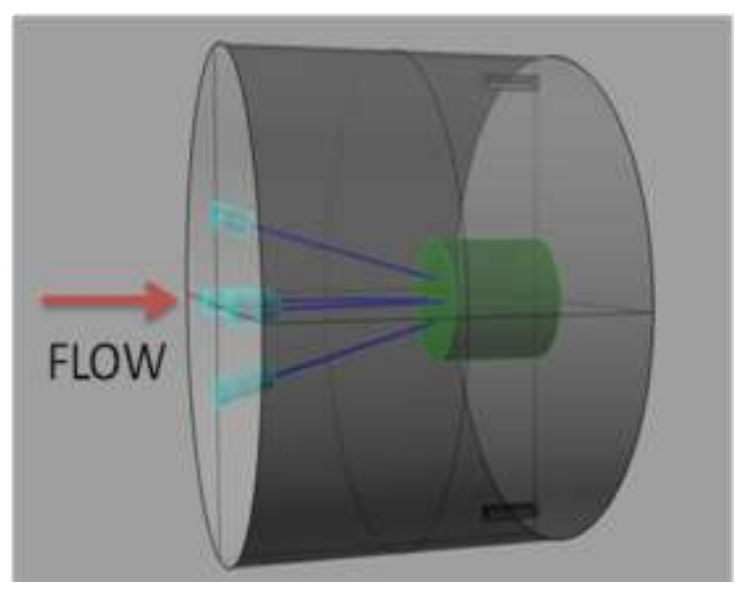

Figure A6. Ghosted view of the test coupon. 


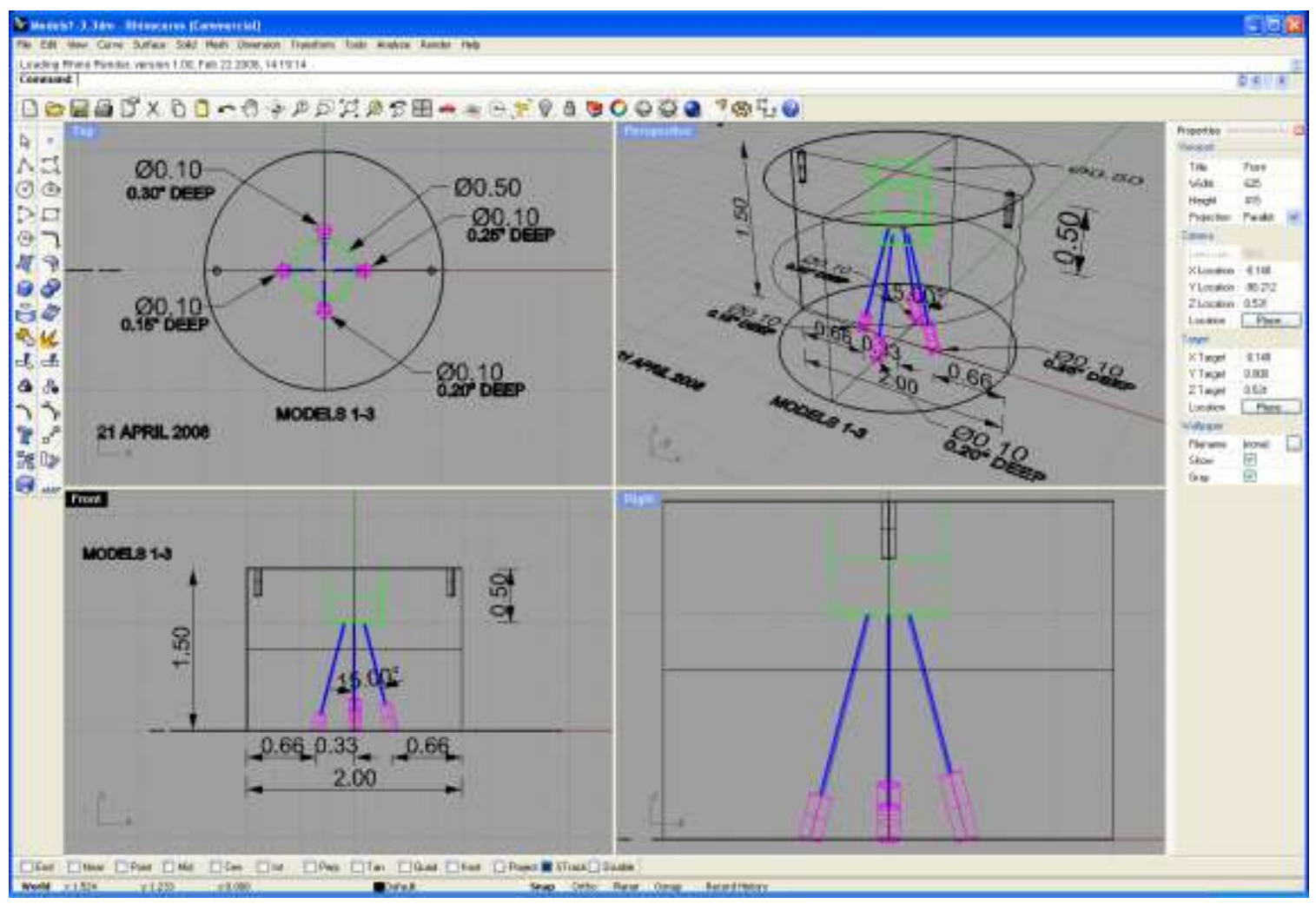

Figure A7. Model design specifications. 


\section{Instrumentation}

The spectrometer used was a commercial off the shelf Czerny-Turner configured Ocean Optics HR4000 high resolution unit (Figure A8). The grating has 300 lines/mm. A $10 \mu \mathrm{m}$ slit provides a spectral resolution of $1.1 \mathrm{~nm}$ full-width-half-max (FWHM). The detector is a Toshiba TCD1304AP linear charged coupled device array with 3636 pixels. The features and acquisition settings for these measurements are listed in Table A-3. The spectrometer was connected to the models through a 25 -foot long, $400 \mu \mathrm{m}$ diameter fiber patchcord (see Figure A9). The fiber was fed into the test chamber through an instrumentation plate and secured in place with a Conax Technologies gland seal. Once the patchcord was in place, it was left throughout the testing and connected to the models using a mating sleeve. The optical fiber models were installed flush at the bottom of counterbores using a high temperature adhesive. After a 48 hour cure the test coupons were mounted inside of the HyMETS test chamber. The spectrometer software was set up for external triggering by the rising sting position signal and worked properly during pre-run testing. However, the first attempt to collect data for a run failed due to premature triggering on noise which left insufficient time to reset the software before the model was inserted into the flow. The external trigger was abandoned and a manual direct signal communication was used.

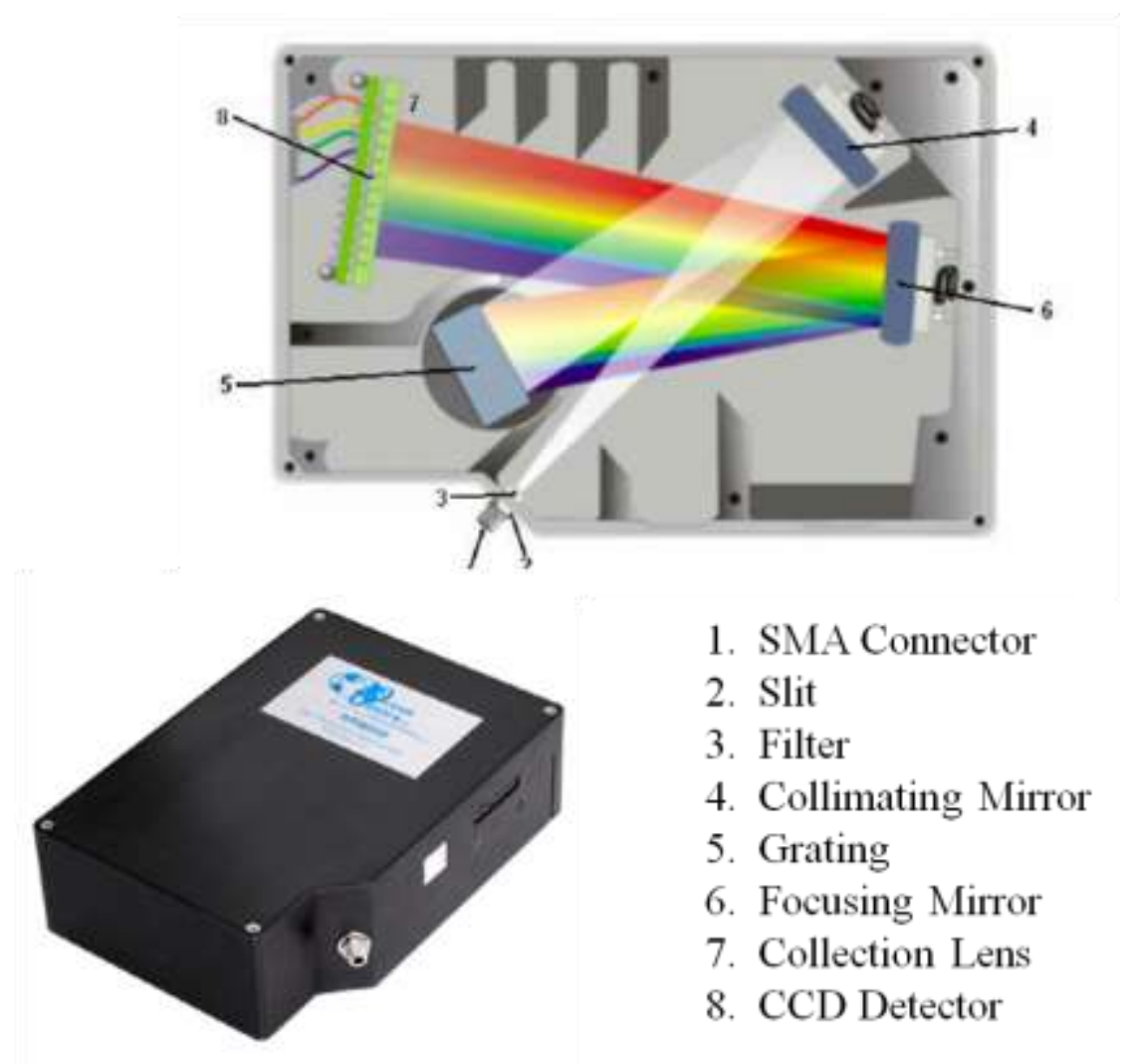

Figure A8. Ocean Optics HR4000 high resolution spectrometer and optical bench. 
Table A-3: Optical spectrum analyzer HR-4000

\begin{tabular}{|c|c|}
\hline Detector & Toshiba TCD1304AP linear CCD, 3636 pixels \\
\hline Effective Range & $10 \mu \mathrm{m}$ \\
\hline Slits & SMA 905 \\
\hline Connector & $300 \mathrm{gr} / \mathrm{mm}$ \\
\hline Grating & $0.02-8.4 \mathrm{~nm}$ FWHM (1.1 nm ) \\
\hline Resolution & $300: 1($ at full signal $)$ \\
\hline Signal-to-noise & $<0.06 \%$ at $600 \mathrm{~nm}$ \\
& $<0.10 \%$ at $435 \mathrm{~nm}$ \\
\hline Stray light & $3.8 \mathrm{~ms}-10 \mathrm{~s}(4 \mathrm{~ms})$ \\
\hline Integration time & $148.6 \times 104.8 \times 45.1 \mathrm{~mm}$ \\
\hline Dimensions & $570 \mathrm{grams}$ \\
\hline Weight & \\
\hline
\end{tabular}




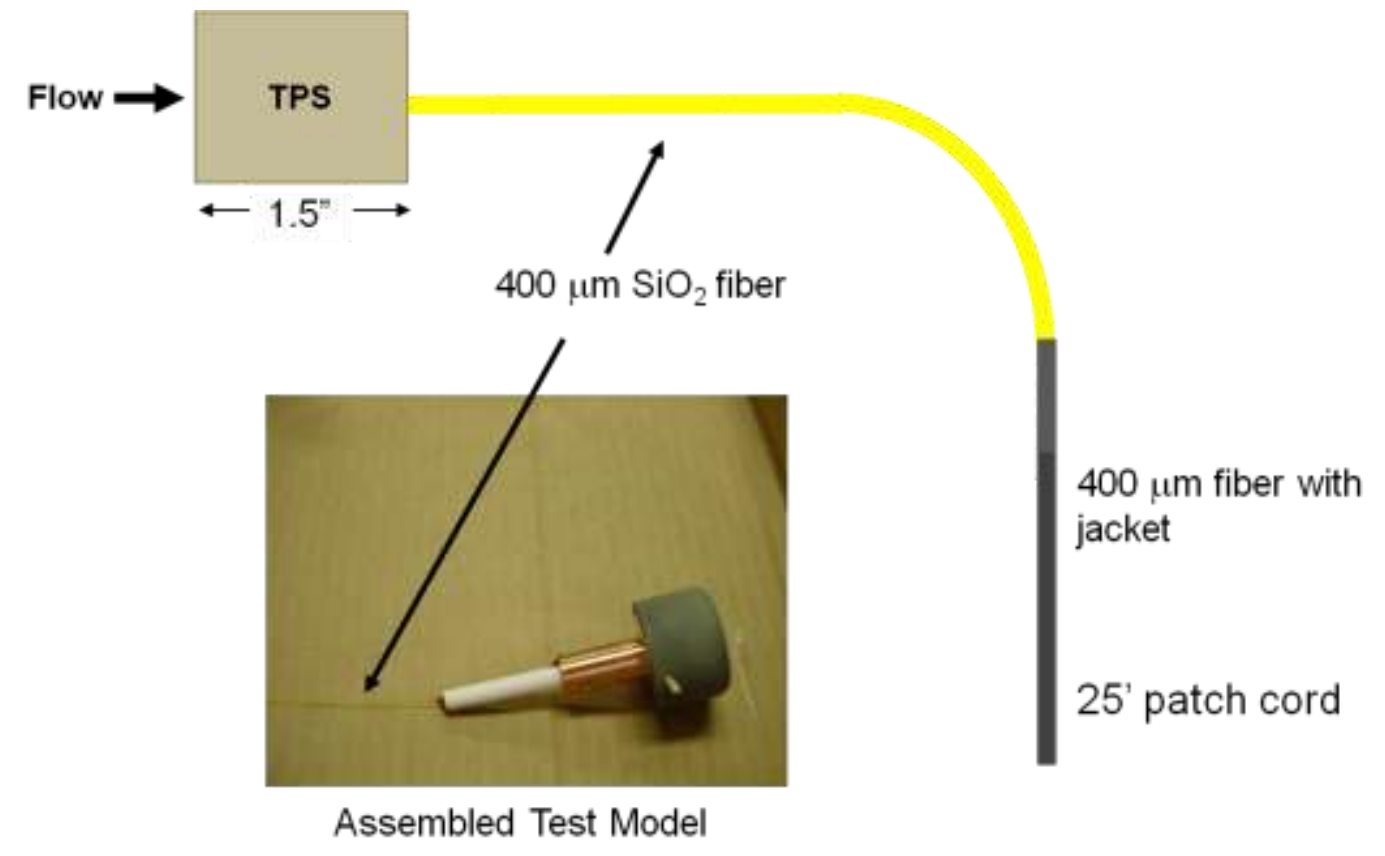

Figure A9. Test model schematic. Inset is a photograph of an assembled test model. 


\section{High Temperature Optical Fiber Measurements}

A total of 8 different models were cycled in the HyMETS facility. Each model consisted of four optical access ports which held the optical fiber. Table A-4 provides a description each test model. The type of TPS material and the configuration including the port size and diameter of the optical fiber are listed in the table. Model 8 incorporated a $0.5 \mathrm{~mm}$ sapphire window. The window was mounted recessed in between the PICA and the fiber. Model 8 was not cycled in the arc jet.

Pre-HyMETS experimentation was critical for successful runs in the arc jet. Light levels were a primary importance. Sufficient light levels were needed to record signal yet, too high light levels would have the potential to damage the spectrometer. A Hamamastu UV-vis PIN detector was used to characterized ambient light levels throughput. In the flow, light detected by the optical fiber passed through a repeatable two-peak pattern before the fiber failed. Figure A10 is plot of voltage versus time for Model 3, 6, and 7. The first peaks occurs $\sim 3$ seconds in the flow. The signal then drops by $50 \%$ over the next 20 seconds. The signal increases to nearly twice the initial peak voltage. After 60 seconds, noise dominates any remaining signal prior to fiber degradation. Fibers cycled at $700 \mathrm{BTU} / \mathrm{ft}^{2}$ lasted approximately $75 \%$ longer than fiber cycled at $550 \mathrm{BTU} / \mathrm{ft}^{2}$. The spectra acquired for Model 4 at 5, 30, and 60 seconds in the flow are shown in Figure A11. The Figure A11 inset shows the model in real-time in the arc jet. Optical spectra were acquired from 200-1100 nm wavelength range. The intensity of the blackbody radiation spectrum increases with time in the flow. The signal is lost due to fiber degradation and melting after keeping the test coupon in the flow for $60 \mathrm{~s}$. This time is sufficient for in-flight signal collection. Gaseous species are clearly identified in the Figure A12. Oxygen, Nitrogen, and traces of Argon emissions are detected. Many of the $\mathrm{N}$ atom transitions have fine structure and nearby lines. The 1.1 $\mathrm{nm}$ spectrometer resolution enabled detection of these lines as shown in Figure A12 with the strongest peak intensity lines labeled. Low intensity peaks at 325 and $328 \mathrm{~nm}$ are possibly due to traces of copper in the flow due to some oxidation of the electrodes. Peaks at 384 and $387 \mathrm{~nm}$ have been observed form CN-violet transitions. 
Table A-4: Model Configuration.

\begin{tabular}{|c|c|c|c|c|c|}
\hline Model & $\begin{array}{c}\text { TPS } \\
\text { Material }\end{array}$ & $\begin{array}{c}\text { Optical Access } \\
\text { Port } 1\end{array}$ & $\begin{array}{c}\text { Optical Access } \\
\text { Port } 2\end{array}$ & $\begin{array}{c}\text { Optical Access } \\
\text { Port } 3\end{array}$ & $\begin{array}{c}\text { Optical Access } \\
\text { Port } 4\end{array}$ \\
\hline 1 & PICA & $\begin{array}{l}300 \mu \mathrm{m} \text { fiber; } \\
0.1 \text { dia.; } 0.10 \\
\text { depth }\end{array}$ & $\begin{array}{c}300 \mu \mathrm{m} \text { fiber; } 0.1 \\
\text { dia.; } 0.20 \text { depth }\end{array}$ & $\begin{array}{c}300 \mu \mathrm{m} \text { fiber; } 0.1 \\
\text { dia.; } 0.30 \text { depth }\end{array}$ & $\begin{array}{c}300 \mu \mathrm{m} \text { fiber; } 0.1 \\
\text { dia.; } 0.40 \text { depth }\end{array}$ \\
\hline 2 & PICA & $\begin{array}{c}300 \mu \mathrm{m} \text { fiber; } .10 \\
\text { dia; } .20 \text { depth }\end{array}$ & OPEN & OPEN & OPEN \\
\hline 3 & PICA & $\begin{array}{c}400 \mu \mathrm{m} \text { fiber; } .10 \\
\text { dia; } \\
0.10 \text { depth }\end{array}$ & $\begin{array}{c}400 \mu \mathrm{m} \text { fiber; } .10 \\
\text { dia; } 0.20 \text { depth }\end{array}$ & $\begin{array}{c}400 \mu \mathrm{m} \text { fiber; } .10 \\
\text { dia; } 0.30 \text { - depth }\end{array}$ & $\begin{array}{c}400 \mu \mathrm{m} \text { fiber; } 10 \\
\text { dia; } 0.40 \text { depth }\end{array}$ \\
\hline 4 & PICA & $\begin{array}{c}600 \mu \mathrm{m} \text { fiber; } .07 \\
\text { dia; } \\
.10 \text { depth }\end{array}$ & $\begin{array}{c}600 \mu \mathrm{m} \text { fiber; } .07 \\
\text { dia; } .20 \text { depth }\end{array}$ & $\begin{array}{c}600 \mu \mathrm{m} \text { fiber; } .05 \\
\text { dia; } .30 \text { depth }\end{array}$ & $\begin{array}{c}600 \mu \mathrm{m} \text { fiber; } .05 \\
\text { dia; } .40 \text { depth }\end{array}$ \\
\hline 5 & PhenCarb & $\begin{array}{c}600 \mu \mathrm{m} \text { fiber; } .07 \\
\text { dia; } .10 \text { depth }\end{array}$ & OPEN & OPEN & OPEN \\
\hline 6 & PhenCarb & $\begin{array}{c}400 \mu \mathrm{m} \text { fiber; } .10 \\
\text { dia; } .15 \text { depth }\end{array}$ & $\begin{array}{c}400 \mu \mathrm{m} \text { fiber; } .10 \\
\text { dia; } .20 \text { depth }\end{array}$ & $\begin{array}{c}400 \mu \mathrm{m} \text { fiber; } .10 \\
\text { dia; } .25 \text { depth }\end{array}$ & $\begin{array}{c}400 \mu \mathrm{m} \text { fiber; } .10 \\
\text { dia; } .30 \text { depth }\end{array}$ \\
\hline 7 & PhenCarb & $\begin{array}{c}600 \mu \mathrm{m} \text { fiber; } .10 \\
\text { dia; } .15 \text { depth }\end{array}$ & $\begin{array}{c}600 \mu \mathrm{m} \text { fiber; } .10 \\
\text { dia; } .20 \text { depth }\end{array}$ & $\begin{array}{c}600 \mu \mathrm{m} \text { fiber; } .10 \\
\text { dia; } .25 \text { depth }\end{array}$ & $\begin{array}{c}600 \mu \mathrm{m} \text { fiber; } .10 \\
\text { dia; } .30 \text { depth }\end{array}$ \\
\hline 8 & PICA & $\begin{array}{c}300 \mu \mathrm{m} \text { fiber; } .10 \\
\text { dia; } .29 \text { depth } \\
\text { Sapphire Window }\end{array}$ & OPEN & OPEN & OPEN \\
\hline 9 & PhenCarb & $\begin{array}{c}400 \mu \mathrm{m} \text { fiber; } .10 \\
\text { dia; } .10 \text { depth }\end{array}$ & $\begin{array}{c}400 \mu \mathrm{m} \text { fiber; } .10 \\
\text { dia; } .20 \text { depth }\end{array}$ & $\begin{array}{l}400 \mu \mathrm{m} \text { fiber; } .10 \\
\text { dia; } .30 \text { depth }\end{array}$ & $\begin{array}{l}400 \mu \mathrm{m} \text { fiber; } .10 \\
\text { dia; } .40 \text { depth }\end{array}$ \\
\hline
\end{tabular}




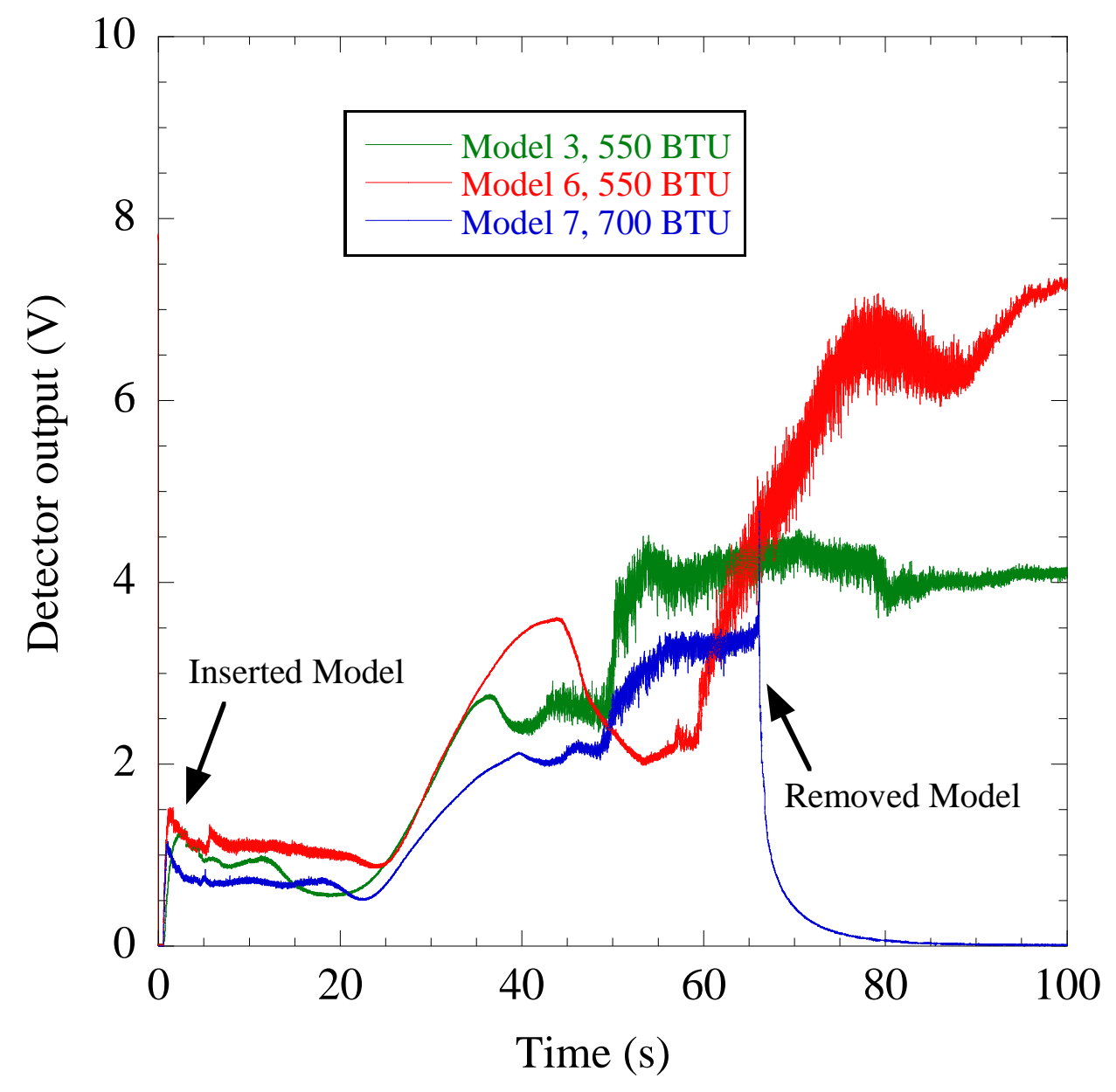

Figure A10. Voltage versus time for Model 3, Model 6, and Model 7. 


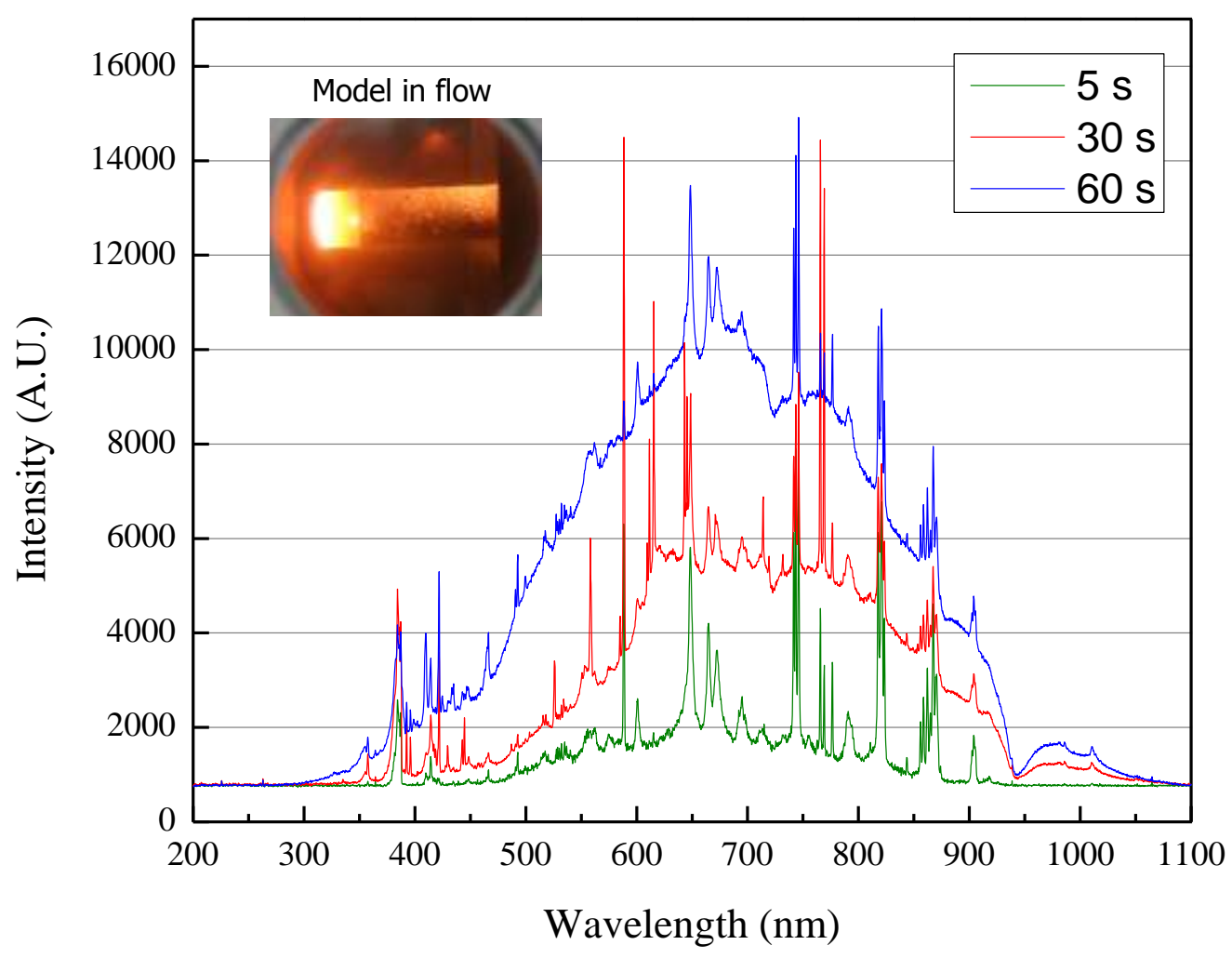

Figure A11. Intensity versus wavelength for Model 4. 


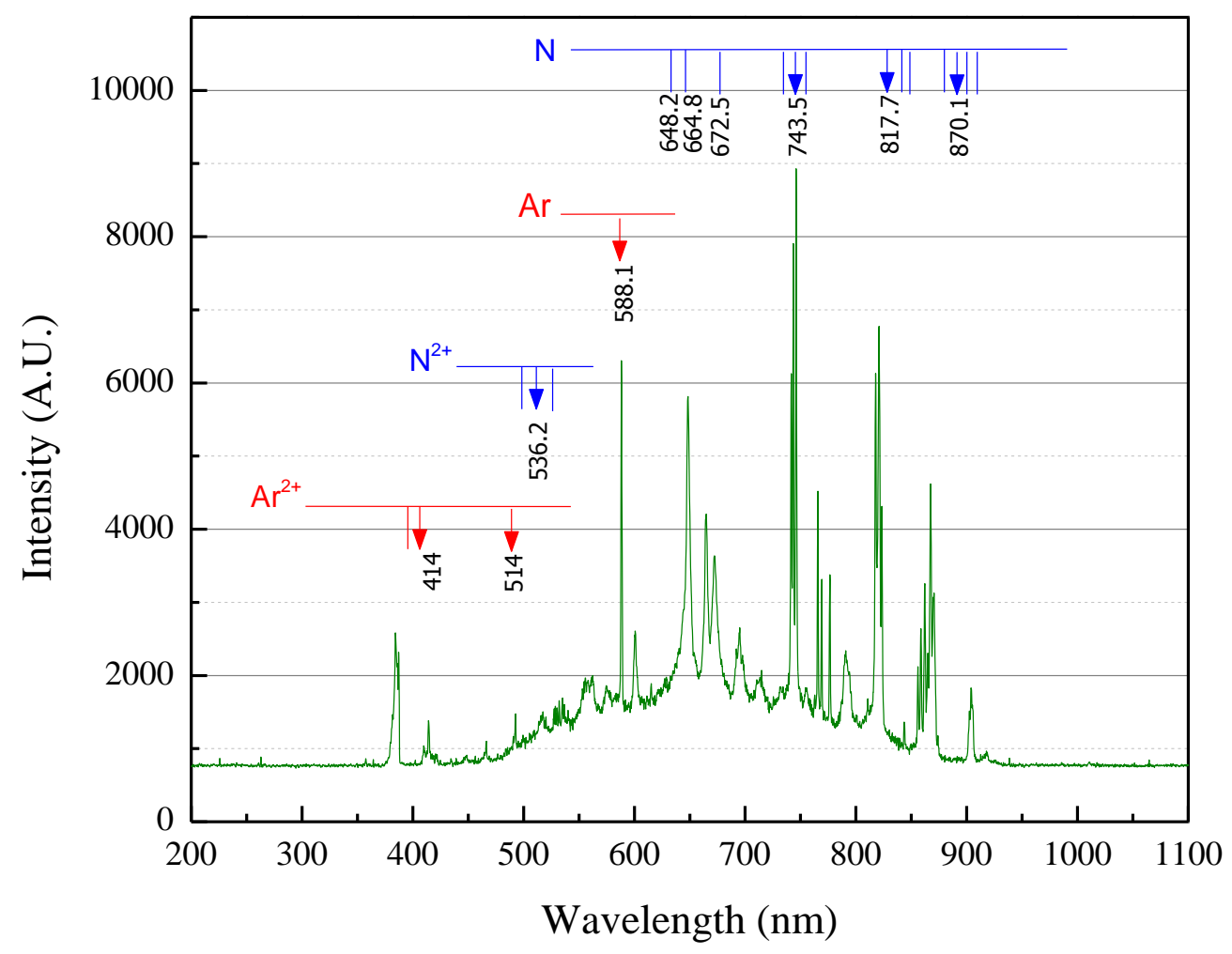

Figure A12. Emission spectra for Earth reentry simulants. 


\section{Conclusions}

A fiber-based approach to obtaining optical spectra of a glowing bow shock in highenthalpy air flow is demonstrated. The testing was carried out on ground in the NASA Langley HyMETS Facility. Commercial optical fiber was embedded into the simulatednose of an ablating blunt-body reentry test model. The design provided direct line-ofsight view into the flow. Baseline data on fused silica optical fiber embedded in TPS were collected in the runs. Results show that embedding optical fiber in the recessedgeometry supports the optical conduit and allows for signal collection under simulated flight conditions. The results show that $600 \mu \mathrm{m}$ test prototypes remained transmissive over the spectral wavelength-region of interest. Fibers in holes 0.05 inch diameter at 0.11 inch depth lasted approximately 50\% longer than fiber embedded with 0.10 diameter at 0.22 in depth. The models maintained structrual integrity after $\sim 60$ second exposure to the flow. This study marks the first time in the NASA history in which optical fibers within an ablative TPS material were characterized in a simulation atmospheric reentry environment. The results contribute new data useful for further study to reduce TPS modeling uncertainties. The concept of embedding fiber optics within ablative TPS material has merit and the potential to yield successful implementation into flight-tests.

\section{References}

D. L. Cauchon, "Radiative heating results from the fire 2 flight experiment in a reentry velocity of 11.3 km/s," NASA TM X-1402, July 1967.

C. Park, "Stagnation-point Radiation for Apollo 4," Journal of Thermophysics and Heat Transfer, V 18, p. 349-357, 2004.

C. B. Phillips, M. G. Lovern, S. D. Russell, C. Howlett, D. A. Levin, P. W. Erdman, "Dual-Mode Experiment on Bow-Shock Interactions (DEBI)," Navigation and Applied Sciences, p. 222-227, 2001. E. Martinez, D. Levin, S. G. Bilen, L. Engle, P. Erdman, T.D. Schott, W. C. Kelliher, R. E. Davis, I. A. Carlberg, "Advanced Optical Instrumentation for Crew Exploration Vehicle," Center White Paper, NASA Langley Research Center, 2006.

T. Smith, S. G. Bilen, D. Morris, D. Levin, T. D. Schott, J. Cecconi, K. Page, SBIR-Phase I Final Report: Low-Intrusive Fiber-Optic Plug for Thermal Protection System Materials, 2008.

S. C. Splinter, R. C. Sykes, T. A. Wallace, and J. G. Gragg, NASA LaRC Hypersonic Materials Environmental Test System (HyMETS) Facility, facility presentation, 2007. 


\title{
APPENDIX B \\ Research Proposal Submitted to NASA LaRC C\&I
}

\section{Tailor-made: Quantum Dots for Remote Sensing}

\author{
Devin Pugh-Thomas*, Brian M. Walsh ${ }^{\dagger}$ \\ *NASA Langley Research Center-University of Virginia, Hampton, VA 23681 \\ Phone: (757) 864-3854, Fax (757) 864-8828, E-mail: devin.pugh-thomas@nasa.gov \\ ${ }^{\dagger} N A S A$ Langley Research Center, Hampton, VA 23681 \\ Phone: (757) 864-7112, Fax (757) 864-8828, E-mail: brian.m.walsh@nasa.gov
}

New NASA exploration pathways to remote sensing, renewable energy, and novel flight sensors are possible with quantum dots. Quantum dots (QDs) are distinguished from all other nanoparticles by their characteristics of size and composition-dependent luminescence. Advances in the science of these quantum-confined nanoparticles will pioneer low cost, light weight, low power communication and sensing in extreme environments. In theory, QDs exhibit potential to rival traditionally used rare-earth ions and transition metal ions as optically active centers for opto-electronic sensing. II-VI, III$\mathrm{V}$, and IV-VI semiconductor QDs with size dependent emission wavelengths from UVVIS to FIR can be tailored for optical sensing and high temperature aerospace applications on NASA missions.

Today, sophisticated synthesis methods yield quantum dots with precisely controlled size distribution. The main hurdle to utilizing QDs in devices is twofold: First, how to matrix quantum dots into a media to yield a thermally stable, rigid, optically transparent nanocomposite. Secondly, what is the physics framework that supports the data? Research on the temperature dependent spectroscopic properties of quantum dots has centered on the detection of lower energy states for temperatures from 15 to $300 \mathrm{~K}$. However, for optoelectronic device development, this range is too low. The behavior of quantum dots at elevated temperatures and explanation of these processes should be investigated to support the development of novel sensors with unique spectral signatures.

We propose a systematic spectroscopic study on optically transparent matrices loaded with quantum dots from the low to high temperature regime. The fundamental physics and supporting measurements on QD exciton interactions and decay kinetics at elevated temperature is missing in the literature. The proposed measurements and analysis will advance the science required for rational design of QD-based materials for remote sensing. The insensitivity of the QD emission to oxygen and certain chemical species presages these materials for aerospace applications. Hence, we hypothesize that by combining QD luminescent indicators, tailor-made fluorescence-based sensors can be realized. 
The proposed research involves intensive experimental work, including the measurement of absorption, photoluminescence, excitation, and lifetime over a wide range of temperatures. The laboratories at NASA Langley have the specialized equipment to support low to high temperature regime optical spectroscopy. A 5-10 K funding will support quantum dot materials and laser beam delivery and signal collection components.

\section{Outcome of Proposal Submission:}

Awarded Funding Amount: \$9,100.00

Materials Procured: -Chemistry laboratory supplies

-Optical signal collection components

-Semiconductor quantum dots 


\section{APPNDIX $\mathbf{C}$ \\ Publications}

[1] D. Pugh-Thomas, B. M. Walsh, M. C. Gupta, "CdSe(ZnS) nanocomposite luminescent high temperature sensor," Nanotechnol., 22, 185503 (2011).

[2] D. Pugh-Thomas, M. C. Gupta, and B. M. Walsh, "Quantum Dots for High Temperature Sensing," Technical digest of the Conference on Lasers and ElectroOptics, Baltimore, Maryland, May 1-6, 2011, paper JWA58.

[3] D. Pugh-Thomas, B. M. Walsh, M. C. Gupta, "Spectroscopy of $\mathrm{BeAl}_{2} \mathrm{O}_{4}: \mathrm{Cr}^{3+}$ with application to high temperature sensing," Appl. Opt., 15, 2891-2897 (2010).

[4] D. Pugh-Thomas, B. M. Walsh, M. C. Gupta, "Absorption cross section and temperature dependent optical properties of CdSe/ZnS quantum dots," Submitted to Applied Optics.

[5] D. Pugh-Thomas and M. C. Gupta, "CdSe/ZnS: $\mathrm{SiO}_{2}$ Optical Fiber Thermal Sensor," Under preparation, 2012.

[6] D. Pugh-Thomas, B. M. Walsh, and M. C. Gupta, "Quantum Dot Quantum Efficiency Measurements,” Under preparation, 2012. 


\title{
$\mathrm{CdSe}(\mathrm{ZnS})$ nanocomposite luminescent high temperature sensor
}

\author{
Devin Pugh-Thomas ${ }^{1,2}$, Brian M Walsh ${ }^{2}$ and Mool C Gupta ${ }^{1}$ \\ ${ }^{1}$ Charles L Brown Department of Electrical and Computer Engineering, \\ University of Virginia, Charlottesville, VA 22904, USA \\ ${ }^{2}$ NASA Langley Research Center, Hampton, VA 23681, USA \\ E-mail: mgupta@ virginia.edu
}

Received 18 December 2010, in final form 14 February 2011

Published 22 March 2011

Online at stacks.iop.org/Nano/22/185503

\begin{abstract}
High temperature luminescence-based sensing is demonstrated by embedding colloidal $\mathrm{CdSe}(\mathrm{ZnS})$ quantum dots into a high temperature $\mathrm{SiO}_{2}$ dielectric matrix. The nanocomposite was fabricated by a solution process method. As-prepared $\mathrm{CdSe}(\mathrm{ZnS})$ quantum dots in the nanocomposite sensor show an absorption band at a wavelength of $600 \mathrm{~nm}(2.06 \mathrm{eV})$. Photoluminescence (PL) measurements show a room temperature emission peak at $606 \mathrm{~nm}$ $(2.04 \mathrm{eV})$. The temperature-dependent emission spectra study shows for the first time a $\mathrm{CdSe}(\mathrm{ZnS})-\mathrm{SiO}_{2}$ nanocomposite-based high temperature sensor. The temperature-dependent spectral and intensity modes of the nanocomposite thin film photoluminescence were investigated from $295-525 \mathrm{~K}$. The sensor shows a variation of the emission wavelength as a function of temperature with a sensitivity of $\sim 0.11 \mathrm{~nm}{ }^{\circ} \mathrm{C}^{-1}$. The film morphology and roughness are characterized using AFM.
\end{abstract}

(Some figures in this article are in colour only in the electronic version)

\section{Introduction}

Colloidal CdSe quantum dots have been highlighted as one of the most distinguished photonic materials in nanotechnology. CdSe semiconductor nanomaterials are of interest to researchers for various reasons. One driver for such research is the potential for the application of quantum dots (qdots) in next-generation electronic and photonic devices [1]. Due to quantum confinement, q-dots have unique optical properties that resemble those of single molecules. $\mathrm{CdSe}(\mathrm{ZnS})$ quantum dots exhibit nearly full visible-range light emission with size variation. Narrow fluorescence emission, relatively high quantum yield, and outstanding photostability have been reported for $\mathrm{CdSe}(\mathrm{ZnS}) \mathrm{q}$-dot solutions and thin films. The ability to tailor the optical properties by changing the particle size gives these materials the potential to solve many of the challenges with luminescence-based temperature sensors. For example, it has been proposed to use an alexandrite crystal to measure temperature [2, 3]. Zhang et al reports data from room temperature up to $900 \mathrm{~K}$ [4]. Reproducible intensity-based sensing with alexandrite has recently been shown to be limited to $\sim 550 \mathrm{~K}$ [5]. Investigations on other transition metal ion and rare-earth doped bulk crystals have reported luminescence- based high temperature sensing [6]. Higher quantum efficiency due to an increase in oscillator strength give quantum dots the potential to rival traditionally used rare-earth ions as optically active centers for thermal sensing. The insensitivity of the emission to oxygen has established $\mathrm{CdSe}(\mathrm{ZnS})$ quantum dots as an attractive class of optical indicators for luminescence thermometry.

A step towards developing useful low cost opto-sensing devices consists of immobilizing quantum dots in solid support structures. Optically transparent matrices containing quantum dots are desired for opto-sensing applications. The temperature-dependent emission of $\mathrm{CdSe}$ and $\mathrm{CdTe}$ q-dots has recently been reported for low temperature ranges (below $300 \mathrm{~K}$ ), thus opening new possibilities for optical temperature sensing devices [7, 8]. When embedded into select matrices, quantum dot-clusters offer stabilized emission, protection from oxygen, and prevention from deposits that interfere with the luminescence. For high temperature applications, the matrix must not impede the luminescence and needs to be chemically and thermally stable. We show that modified silicon dioxide, which is solution processable, fulfills these requirements. To the best of our knowledge, the high temperature dependence of q-dot nanoparticle luminescence 


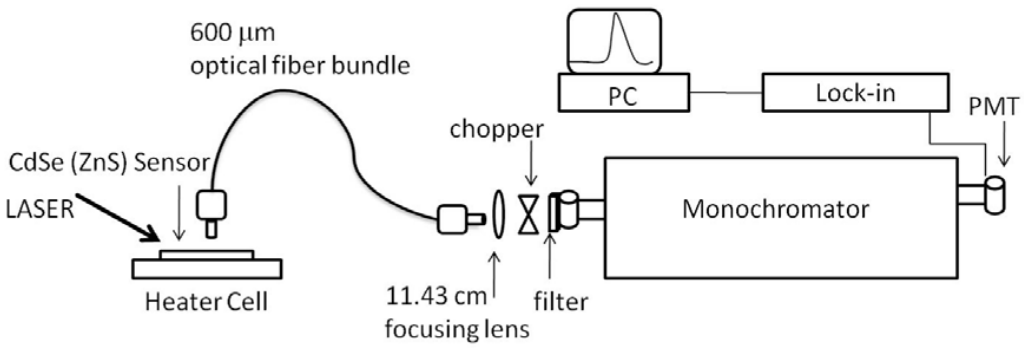

Figure 1. Experimental setup used to characterize the temperature sensor.

in a high temperature resistant matrix, which is important to many thermometry applications, has not been previously reported. In this work, $\mathrm{CdSe}(\mathrm{ZnS}): \mathrm{SiO}_{2}$ luminescence-based high temperature optical sensing is demonstrated for the first time. Extensive research into quantum dots of II-VI compound semiconductors, particularly $\mathrm{CdSe}$, is due to the greater possibility of bandgap tuning for obtaining desired optoelectronic properties. Bulk CdSe has a direct intrinsic bandgap of $1.74 \mathrm{eV}$. On the nanoscale, the q-dot bandgap can be tuned across the visible, making CdSe an interesting material for light-emitting diodes, bio-labeling, and optosensors [9]. The photoluminescent emission of CdSe has been shown to be enhanced by surface passivation with a larger bandgap material such as $\mathrm{ZnS}(2.72 \mathrm{eV})$ [10]. Encapsulating the nanocrystal core gives quantum yields on the order of $50 \%$ larger. These factors motivated this research toward a luminescence-based $\mathrm{CdSe}(\mathrm{ZnS})$ high temperature sensor.

\section{Experimental details}

\subsection{General}

The nanocomposite thin films were solution-fabricated and the temperature-sensitive parameters were characterized by optical spectroscopy. High temperature resistant $\mathrm{SiO}_{2}$ solution was purchased from Filmtronics Materials. Ocean Nanotechnology Company supplied the quantum dots. Core shell $\mathrm{CdSe}(\mathrm{ZnS})$ quantum dots were synthesized by colloidal growth solution phase processing. The nanocrystals were overcoated with zinc sulfide to yield stable and highly emissive $\mathrm{CdSe}(\mathrm{ZnS})$ quantum dots.

\subsection{QD nanocomposite sensor fabrication}

A variety of techniques like spray pyrolysis, thermal decomposition, molecular beam epitaxy, chemical vapor deposition, and laser ablation have been widely used in the synthesis of nanomaterials and nanostructures. In this work, $\mathrm{CdSe}(\mathrm{ZnS}): \mathrm{SiO}_{2}$ nanocomposite thin films were fabricated by spin coating and heat treatment. The QD powder was measured to $10 \mathrm{mg}$ and added in the $\mathrm{SiO}_{2}$ solution. Dispersion of the qdots in the $\mathrm{SiO}_{2}$ solution was carried out by sonicating for $1 \mathrm{~h}$ at $300 \mathrm{~K}$. The film was formed by dropping $50 \mu 1$ of $\mathrm{SiO}_{2}$-QD solution onto a stationary $1.27 \mathrm{~cm}$ diameter quartz substrate followed by spin coating for $30 \mathrm{~s}$ at $1000 \mathrm{rpm}$. The films were then subjected to heat treatment in air. Thermal treatment yields a compact glass layer $\sim 1 \mu \mathrm{m}$ thick that protects the nanoparticles from oxidation during characterization.

\subsection{Sensor characterization}

After fabrication of the sensors, optical absorption spectra were taken using a Cary $5 \mathrm{E}$ spectrophotometer. Atomic force microscopy (AFM) images were obtained in the acoustic AC mode on a TopoMetrix Explorer AFM. The height profile analysis was carried out using WSxM software [11]. The temperature-dependent emission of the sensor was performed using the experimental setup shown in figure 1. In order to study the temperature response the sensor was mounted into a custom built high temperature cell. The heating element used was a Watlow ULTRAMIC 600 ceramic heater. A 532-AP Omnichrome Argon laser at $514 \mathrm{~nm}$ operating at an output power of $35 \mathrm{~mW}$ was used as the excitation source for the luminescence measurements. The luminescence was focused by a $5 \mathrm{~cm}$ lens into a $600 \mu \mathrm{m}$ optical fiber. The signal was modulated by a chopper placed at the entrance to the monochromator. The collected luminescence was focused onto the $100 \mu \mathrm{m}$ slit of a $0.5 \mathrm{~m}$ SPEX monochromator. A $0.10 \mathrm{~nm}$ spectral resolution was obtained with a $100 \mu \mathrm{m}$ slit width. A $1200 \mathrm{~g} \mathrm{~mm}^{-1}$ grating blazed at $0.75 \mu \mathrm{m}$ was used in all measurements. An RG580 long pass glass filter blocked the pump beam. An Oriel 77348 photomultiplier tube detected the signal. Data was acquired using an SRS 530 lock-in amplifier coupled to a computer. Data storage and processing were accomplished using the SRS/Cd2A data acquisition computer program [12].

\section{Results and discussion}

The optical absorption spectra of the $\mathrm{CdSe}(\mathrm{ZnS}): \mathrm{SiO}_{2}$ thin film nanocomposite and solution are shown in figure 2 . The spectra in figures 2(a) and (b) are of 1 and $5 \mu \mathrm{m}$ thick films. The absorption peaks are due to the quantum dots, as reported in room temperature measurements and theoretical studies by Ekimov et al and Woggon et al [13, 14]. The spectra show several peaks corresponding to different optical transitions. The first three quantum dot resonances were reproduced by the superposition of three Gaussian bands. The first peak dominates at $2.08 \mathrm{eV}$, while the other two peaks show up as a shoulder at $\sim 2.50$ and $\sim 2.76 \mathrm{eV}$. The peaks are due to $1 \mathrm{~S}_{3 / 2} \rightarrow$ $1 \mathrm{~S}_{e}, 2 \mathrm{~S}_{3 / 2} \rightarrow 1 \mathrm{~S}_{e}$ and $1 \mathrm{P}_{3 / 2} \rightarrow 1 \mathrm{P}_{e}$ transitions [13, 14]. 


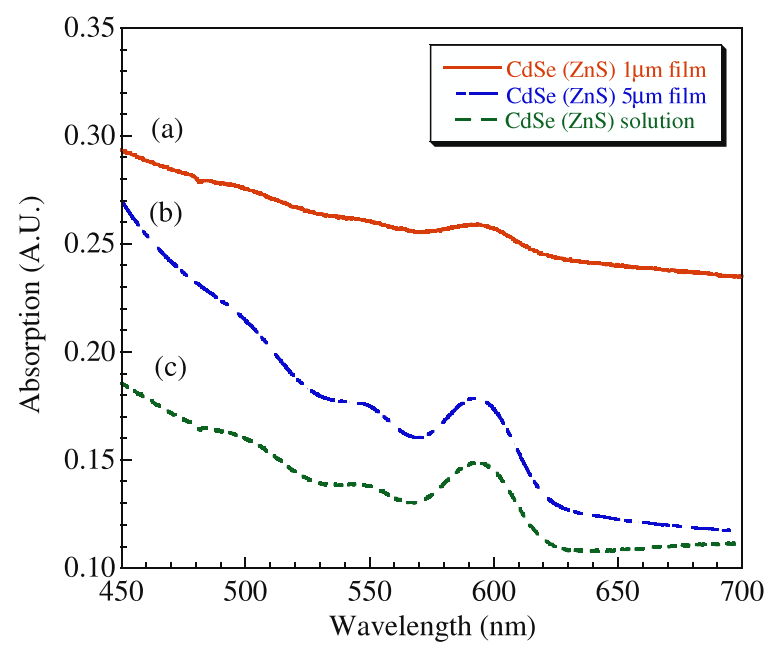

Figure 2. UV-vis absorption spectra of $\mathrm{CdSe}(\mathrm{ZnS}): \mathrm{SiO}_{2}$ quantum dot nanocomposite (a) $1 \mu \mathrm{m}$ film, (b) $5 \mu \mathrm{m}$ film and (c) solution recorded at $T=295 \mathrm{~K}$.

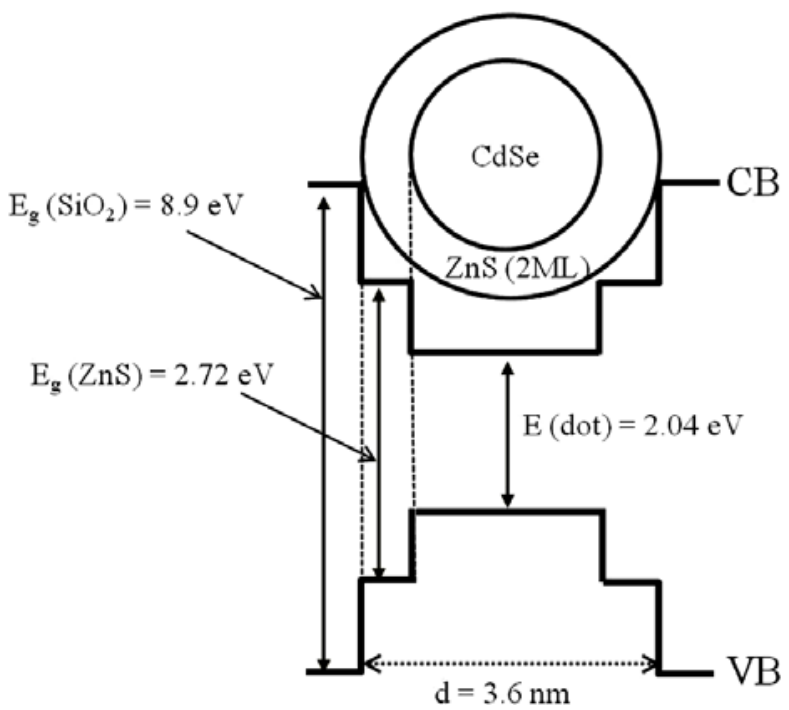

Figure 3. Schematic structure of $\mathrm{CdSe}(\mathrm{ZnS})$ quantum dots.

In bulk CdSe, the direct energy bandgap is $1.74 \mathrm{eV}$ and the Bohr exciton radius is $\sim 9.6 \mathrm{~nm}$. An absorption peak is expected in the bulk at $\sim 700 \mathrm{~nm}$. We observe a strong blue shift in both the solution and sensor optical absorption spectra. This observation indicates that the particles are smaller than the Bohr exciton radius of $\mathrm{CdSe}$ and confirms that the reported observations are in the strong quantum confinement regime. The UV-vis absorption peak positions of q-dots are determined by their bulk energy bandgap and quantum confinement. The latter is observed from particles smaller than the exciton Bohr radius. The size-dependent energy bandgap of semiconductor quantum dots is approximated using the following equation [15]:

$$
E=E_{\mathrm{g}}(\mathrm{bulk})+\frac{h^{2}}{2 m^{*} d^{2}}-\frac{1.8 e^{2}}{2 \pi d \varepsilon \varepsilon_{o}}
$$

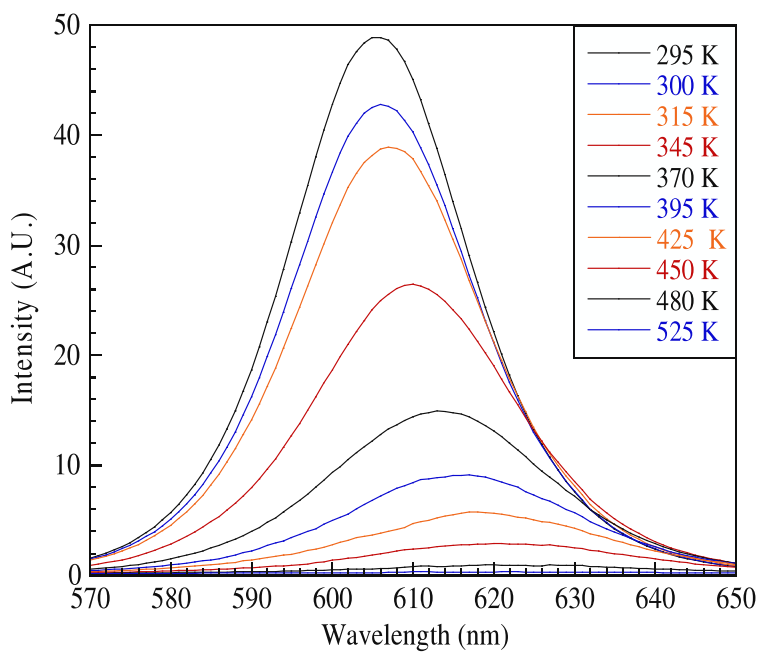

Figure 4. Temperature response of the $\mathrm{CdSe}(\mathrm{ZnS})-\mathrm{SiO}_{2}$ photoluminescence from room temperature to $525 \mathrm{~K}$.

where $E$ and $E_{\mathrm{g}}$ (bulk) are the energy bandgap of the quantum dot nanocrystal and the bulk semiconductor, $h$ is Planck's constant, $d$ is the diameter of the nanocrystal, and $m^{*}$ is the reduced mass of the exciton. The electron charge, dielectric constant, and the space dielectric constant are denoted by $e$, $\varepsilon$, and $\varepsilon_{0}$. The energy band structure of the CdSe q-dot is schematically shown in figure 3 , where $\mathrm{CB}$ identifies the conduction band and VB the valence band. Referring to the vacuum level as potential energy zero, the conduction band of $\mathrm{CdSe}$ is at $-4.95 \mathrm{eV}$ (electron affinity of CdSe). The CdSe q-dots used in this research have a diameter of $\sim 3-4 \mathrm{~nm}$ (per supplier specifications), which includes a 2 monolayer (ML) surface passivation of $\mathrm{ZnS}$. The energy separation between the ground electron state of the conduction band and the ground hole state in the valence band is $2.04 \mathrm{eV}$, corresponding to the emission wavelength at $606 \mathrm{~nm}$. Due to quantum confinement effects in q-dots, electronic states in the conduction band (hole states in the valence band) become quantized as $E_{c 0}, E_{c 1}$ etc ( $E_{v 0}, E_{v 1}$ etc), where $E_{c 0}$ and $E_{v 0}$ denote the ground electron and hole state, respectively. Hence, from the effective mass approximation, the ground state energy levels, $E_{1 \mathrm{~s}}$ are:

$$
E_{1 \mathrm{~s}}=\frac{h^{2}}{2 m^{*} d^{2}}
$$

where $h$ is Planck's constant, $d$ is the quantum dot diameter, and $m^{*}$ is the CdSe reduced mass. As a result of quantum confinement, the effective bandgap, $E_{\mathrm{g}}^{\text {eff }}$ of CdSe quantum dots can be written as:

$$
E_{\mathrm{g}}^{\mathrm{eff}}=E_{\mathrm{g}}(\text { bulk })+\frac{h^{2}}{2 m^{*} d^{2}}
$$

where $E_{\mathrm{g}}$ (bulk) is the bandgap of bulk CdSe. The parameters in the approximation are: the CdSe bulk bandgap is $1.74 \mathrm{eV}$ and the effective mass of electrons and holes are $0.13 m_{\mathrm{o}}$ and $0.45 m_{\mathrm{o}}$, respectively, where $m_{\mathrm{o}}$ is the free electron mass [16]. From (3), the approximate particle radius is calculated to be $3.6 \mathrm{~nm}$. 

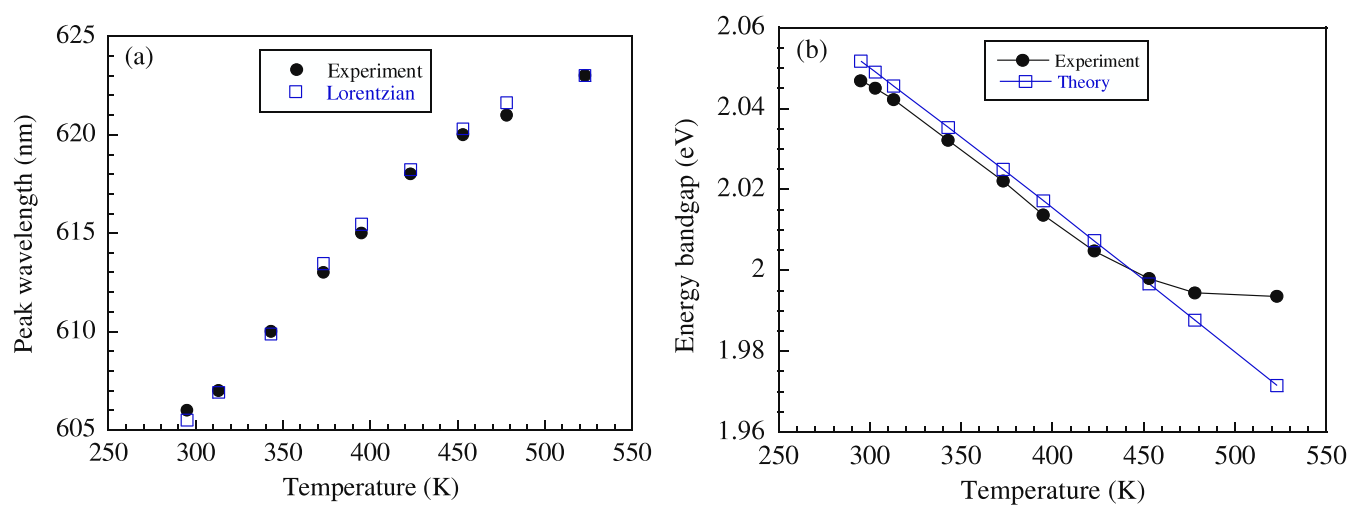

Figure 5. Dependence of the $\mathrm{CdSe}(\mathrm{ZnS})-\mathrm{SiO}_{2}$ (a) emission peak wavelength and (b) energy bandgap with respect to temperature.

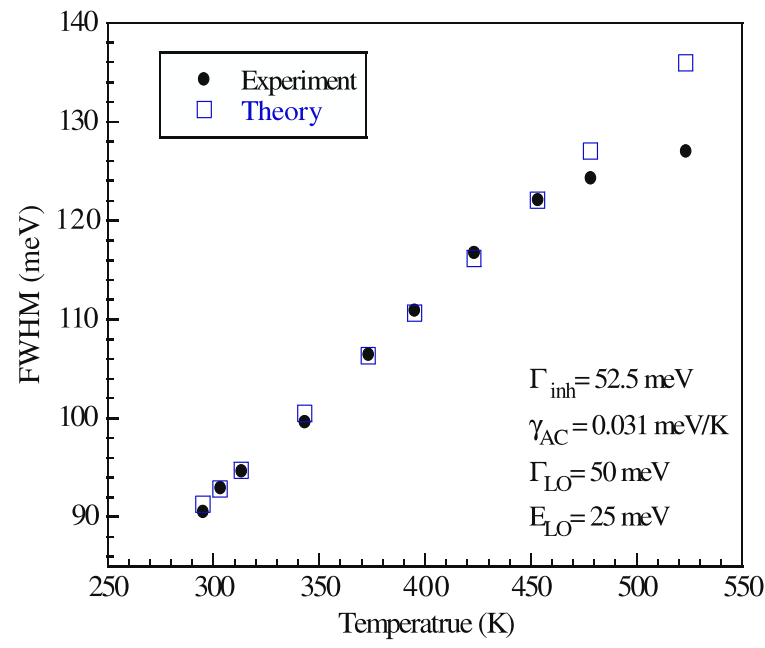

Figure 6. Variation in the full width at half maximum (FWHM) with respect to temperature.

Figure 4 shows the evolution of the luminescence spectra when the temperature is increased from 295 to $525 \mathrm{~K}$. The data were taken for a $2 \mathrm{~h}$ continuous reversible heating process. Under analysis the data are normalized with respect to the room temperature measurement. We observed the PL intensity band red-shift and broaden with increasing temperature. A Lorentzian fit yields a nearly linear change in peak emission wavelength with temperature, as shown in figure 5(a). The wavelength shift can be explained by the fact that heat expands the crystalline network of the q-dot material. The expansion causes a change in the bandgap, which is evident by the red-shift in the emission peak wavelength with increasing temperature. The temperature-dependent bandgap in bulk semiconductor materials is described by the Varshni relation [17]:

$$
E_{\mathrm{g}}(T)=E_{\mathrm{g} 0}-\alpha \frac{T^{2}}{T+\beta}
$$

where $E_{\mathrm{g} 0}$ is the energy gap at $0 \mathrm{~K}$, and $\alpha$ and $\beta$ are material constants termed Varshni coefficients. Specifically, $\alpha$ represents the linear shift in the bandgap with temperature and $\beta$ is near the Debye temperature, $\theta_{\mathrm{D}}$. Results on extension of this empirical relation to semiconductor quantum dots have been reported in the literature [18-20] and are referenced in this work as a benchmark comparison for our new material system. Table 1 compares the fitting parameters from this study to CdSe q-dots embedded in polystyrene (PS), CdSe qdots embedded into a UV resin, and bulk CdSe. Shown in figure 5(b), a best-fit curve with $\alpha=3.8 \times 10^{-4} \mathrm{eV} \mathrm{K}^{-1}$ and $\beta=150 \mathrm{~K}$ is in agreement with the experimental data.

Figure 6 shows the variation of full width at half maximum (FWHM) with temperature. In general, line broadening is composed of inhomogeneous and homogeneous terms. The homogeneous term can be obtained at higher temperatures by fitting the PL spectra with a complex Voigt function. To simplify the calculation, we fit the emission peak with a Lorentzian function for all temperatures. The carrier-phonon processes (exciton scattering with acoustic and longitudinal optical (LO) phonons) that result in the broadening of the FWHM, $\Gamma$, with increasing temperature can be defined by [21]:

$$
\Gamma(T)=\Gamma_{\mathrm{inh}}+\gamma_{\mathrm{AC}} T+\Gamma_{\mathrm{LO}}\left[\exp \left(\frac{E_{\mathrm{LO}}}{k_{\mathrm{B}} T}\right)-1\right]^{-1}
$$

where $\Gamma_{\text {inh }}$ is the inhomogeneous broadening, $\gamma_{\mathrm{AC}}$ is the exciton-acoustic phonon scattering coefficient, $\Gamma_{\mathrm{LO}}$ represents the exciton-LO phonon coupling strength, $E_{\mathrm{LO}}$ is the LOphonon energy, and $k_{\mathrm{B}}$ is the Boltzmann constant. The first

Table 1. Comparison of Varshni coefficients for CdSe/ZnS quantum dots.

\begin{tabular}{lcccl}
\hline Varshni coefficient & This study & Valerini et al $[18]$ CdSe/ZnS:PS & Joshi et al [19] CdSe/ZnS & Hellwege [20] CdSe bulk \\
\hline$\alpha\left(10^{-4} \mathrm{eV} \mathrm{K}^{-1}\right)$ & $3.8 \pm 0.2$ & $3.2 \pm 0.2$ & $4.5 \pm 0.6$ & $2.8-4.1$ \\
$\beta(\mathrm{K})$ & $150 \pm 20$ & $220 \pm 30$ & $195 \pm 70$ & $181-315$ \\
$T(\mathrm{~K})$ & $293-525$ & $50-300$ & $10-300$ & Room temperature \\
\hline
\end{tabular}




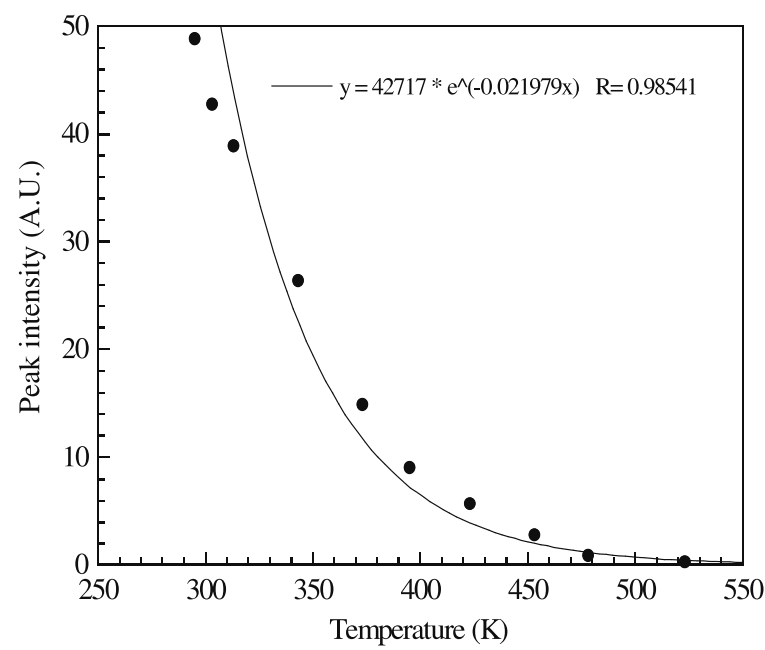

Figure 7. Dependence of the photoluminescence peak intensity with respect to temperature.

term in (5) is due to the size, shape, and composition of the nanoparticles. The other two terms take into account exciton-phonon interactions. $E_{\mathrm{LO}}$ is taken as $25 \mathrm{meV}$ from Raman spectra on CdSe q-dots in previous reports [22, 23]. A good agreement between the experimental data and the bestfit curve (see figure 6) is obtained for $\Gamma_{\text {inh }}=52.5 \mathrm{meV}$, $\Gamma_{\mathrm{LO}}=50 \pm 1 \mathrm{meV}$, and $\gamma_{\mathrm{AC}}=0.031 \mathrm{meV} \mathrm{K}^{-1}$. The excitonLO phonon coupling coefficient is significantly smaller than that of $100 \mathrm{meV}$ in bulk CdSe [18]. Rudin et al estimates a theoretical value of $\gamma_{\mathrm{AC}}=0.06 \times 10^{-3} \mathrm{meV} \mathrm{K}^{-1}$ for bulk CdSe [24]. This value, possibly in error, is significantly smaller than values usually reported for bulk CdSe, which are $\sim 0.008 \mathrm{meV} \mathrm{K}^{-1}[18,25]$. The theory of exciton-phonon interaction in quantum dots predicts a reduced exciton-LO phonon coupling and an enhanced exciton-acoustic phonon interaction [25]. Our results are consistent with theoretical predictions of a strong increase in the coupling with acoustic phonons in zero-dimensional material systems.

The variation in PL intensity with temperature is shown in figure 7. We observe a linear decrease in the evolution of the PL intensity from room temperature up to $373 \mathrm{~K}$. A faster, exponential decrease is observed from 373-525 K. The data in figure 7 is approximated by an exponential with a correlation factor of $R^{2}=0.985$. In general, the PL intensity of quantum dots exponentially decreases with increasing temperature due to thermal quenching. Thermal quenching in core and core/shell dots is attributed to carrier trapping by surface defect states (traps) and thermal escape due to scattering with multiple LO phonons [17, 26, 27]. Furthermore, the ligand shell and the matrix also influence the temperature dependence of the PL intensity. In this study, surface passivation with higher bandgap quantum shell $\mathrm{ZnS}$ electronically isolates the core for stable, maximized PL emission. The $\mathrm{ZnS}$ buries the $\mathrm{CdSe}$ in a potential energy well, concentrating the charge carriers within the nanocrystal core. The $\mathrm{S}^{2-}$ with a much lower oxidation potential than $\mathrm{Se}^{2-}$ results in a higher threshold to photo-oxidative degradation

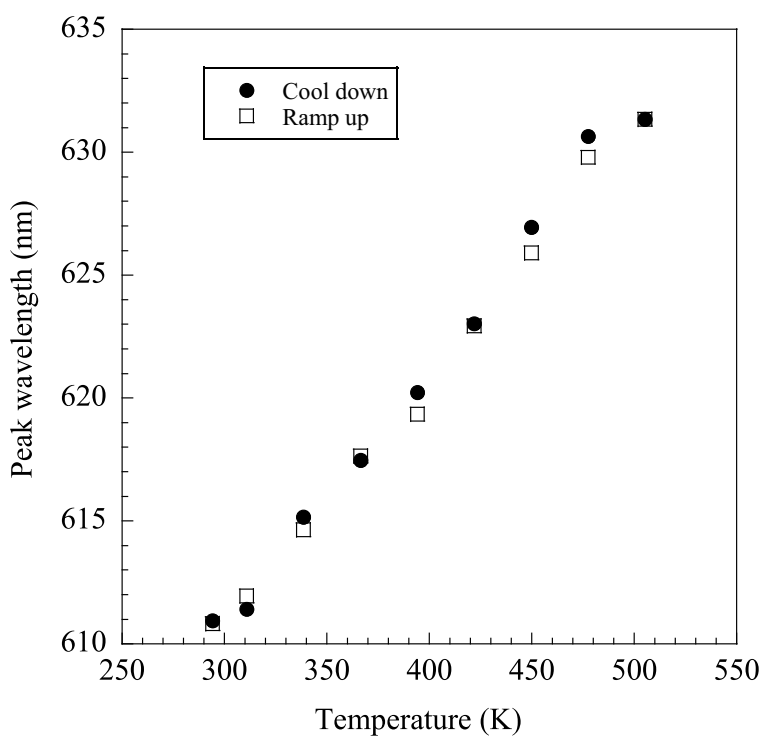

Figure 8. Sensor stability under thermal cycling for the wavelength shift.

and surface defect formation [10]. When embedded into high temperature resistant $\mathrm{SiO}_{2}$ dielectric, extension of $\mathrm{CdSe}(\mathrm{ZnS})$ quantum dots into the high temperature sensing regime is accomplished.

The $\mathrm{CdSe}(\mathrm{ZnS}): \mathrm{SiO}_{2}$ sensor stability was characterized. The wavelength shift under thermal cycle is shown in figure 8 . The sensor recovers the wavelength shift. Relaxation of the strained $\mathrm{SiO}_{2}$ network upon cooling allows for some recovery of the signal at high temperatures. The mechanism of thermal quenching of the PL intensity has been studied for quantum dots at low temperatures $[26,27]$. In this work, the lattice protects the quantum dots, which is observed by the peak wavelength recovery under thermal cycle. The mechanism of PL intensity quenching in $\mathrm{CdSe}(\mathrm{ZnS}): \mathrm{SiO}_{2}$ nanocomposite films at elevated temperatures is under investigation.

Figure 9 shows the film microstructure and line scan obtained by AFM for the as-received $\mathrm{CdSe}(\mathrm{ZnS})$ quantum dots embedded in the thin film matrix. AFM reveals the nanoparticles are dispersed throughout the nanocomposite with an average particle size $\sim 3.6 \mathrm{~nm}$. An absorption maxima at $586 \mathrm{~nm}$ corresponds to a particle diameter of $3.4 \mathrm{~nm}[19,27]$, in good agreement with our absorption peak at $593 \mathrm{~nm}$ and average particle size AFM measurement of $3.6 \mathrm{~nm}$. The isolation and the subsequent resolution of individual dispersed q-dots from a colloidal (cluster) solution are difficult, as extensively reported in the literature $[18,28]$. In this work, the difficulty associated with size selection is possibly due to the aggregation of the dots during solution processing. The cluster effect strongly enhances the PL emission in $\mathrm{CdSe}(\mathrm{ZnS}): \mathrm{SiO}_{2}$, which is a benefit for application to high temperature sensors.

\section{Conclusions}

To realize low cost opto-sensing devices, we synthesized nanocomposite thin films of photochemically and thermally 

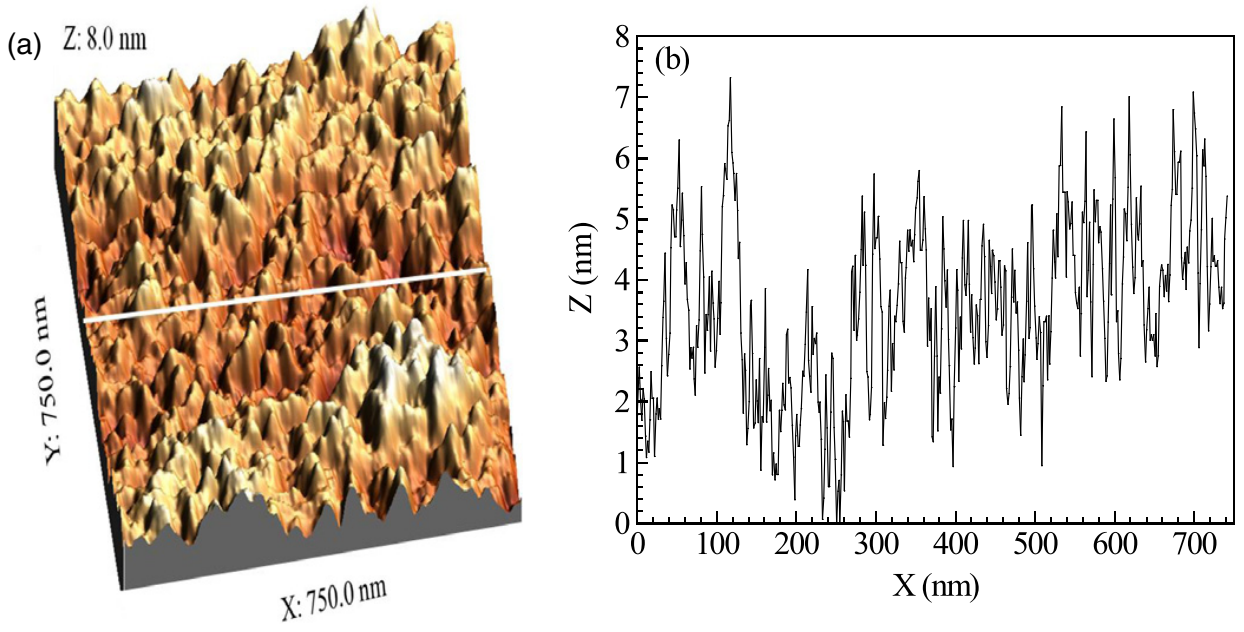

Figure 9. (a) AFM surface topographical images $(0.75 \mu \mathrm{m} \times 0.75 \mu \mathrm{m})$ and (b) line scan of $\mathrm{CdSe}(\mathrm{ZnS}): \mathrm{SiO}_{2}$ film.

stable semiconductor quantum dots. The sensor is solutionfabricated by loading q-dots into a high temperature resistant dielectric matrix. The room temperature optical absorption is measured and the temperature dependence of PL emission is investigated from 295 to $525 \mathrm{~K}$. The emission peak wavelength red-shifts and the FWHM increases with temperature. Varshni's relation is applied to q-dot materials in this high temperature range. The coefficients were determined by thermal property estimation and spectrum analysis to be $\alpha=$ $3.8 \pm 0.2 \times 10^{-4} \mathrm{eV} \mathrm{K}^{-1}$ and $\beta=150 \pm 20 \mathrm{~K}$. The temperature-dependent spectral shift of the q-dots PL emission under thermal cycling enables self-referenced intensity-based temperature measurements with $0.11 \mathrm{~nm}^{\circ} \mathrm{C}^{-1}$ sensitivity. For the first time, we show a $\mathrm{CdSe}(\mathrm{ZnS}): \mathrm{SiO}_{2}$ luminescence-based high temperature sensor. This $\mathrm{CdSe}(\mathrm{ZnS}): \mathrm{SiO}_{2}$ sensor can be applied in combination with other luminescent indicators for multi-sensing applications using the same immobilization chemistry. Analysis of the temperature-dependent PL intensity and its relaxation dynamics is under further study. The dependence of the exciton absorption of $\mathrm{CdSe}(\mathrm{ZnS}): \mathrm{SiO}_{2}$ for high temperature sensing is also being investigated.

\section{Acknowledgments}

The authors thank William C Edwards and William S Luck for their support. We thank NASA/National Institute of Aerospace (NIA) for support through the Langley Professor program.

\section{References}

[1] Konstantatos G and Sargent E H 2010 Nanostructured materials for photon detection Nat. Nanotechnol. 5 391-400

[2] Kobe A, Babnik A and Mozina J 1998 Design aspects of alexandrite fluorescence lifetime fiber optic thermometer Proc. SPIE 3483 45-8

[3] McSherry M, Fitzpatrick C and Lewis E 2005 Review of luminescent based fiber optic temperature sensors Sensors Rev. 25 56-62
[4] Zhang Z, Grattan G T V and Palmer A W 1992 Fiber-optic high temperature sensor based on the fluorescence lifetime of alexandrite Rev. Sci. Instrum. 63 3869-73

[5] Pugh-Thomas D, Walsh B M and Gupta M C 2010 Spectroscopy of $\mathrm{BeAl}_{2} \mathrm{O}_{4}: \mathrm{Cr}^{3+}$ with application to high temperature sensing Appl. Opt. 49 2891-7

[6] Khalid A and Kontis K 2008 Thermographic phosphors for high temperature measurements: principles, current state of the art and recent applications Sensors Actuators 8 5673-744

[7] Walker G W, Sundar V C, Rudzinski C M, Wun D M, Bawendi G and Nocera D G 2003 Quantum-dot optical temperature probes Appl. Phys. Lett. 83 3555-7

[8] de Bastida G, Arregui F J, Goicoechea J and Matias I R 2006 Quantum dots-based optical fiber temperature sensors fabricated by layer-by-layer IEEE Sensors J. 6 1378-9

[9] Bera D, Qian L, Tseng T and Holloway P H 2010 Quantum dots and their multimodal applications: a review Materials 3 2260-345

[10] Smith A M and Nie S 2010 Semiconductor nanocrystals: structures, properties, and band gap engineering Acc. Chem. Res. 43 190-200

[11] Horcas I, Fernandes R, Gomez-Rodriguez J M, Colchero J, Gomez-Herrero J and Baro A M 2007 WSxM: a software for scanning probe microscopy and a tool for nanotechnology Rev. Sci. Instrum. $\mathbf{7 8} 0137051$

[12] Walsh B M SRS/Cd2A Program written by coauthor

[13] Ekimov A I, Hache F, Schanne-Klein M C, Ricard D and Flytzanis C 1993 Absorption and intensity-dependent photoluminescence measurements on CdSe quantum dots: assignment of the first electronic transitions J. Opt. Soc. Am. B 10 100-7

[14] Woggon U, Wind O, Gindele F, Tsitsishvili E and Muller M 1996 Optical transitions in CdSe quantum dots: from discrete levels to broad gain spectra J. Lumin. 70 269-80

[15] Kwak W, Kim T G, Chae W and Sung Y 2007 Tuning the energy bandgap of $\mathrm{CdSe}$ nanocrystals via $\mathrm{Mg}$ doping Nanotechnology 18 1-4

[16] Huibing M, Chen J, Wang J, Li Z, Dai N and Zhu Z 2005 Photoluminescence investigation of CdSe quantum dots and the surface state effect Physica E 27 124-8

[17] Varshni Y P 1967 Temperature dependence of the energy gap in semiconductors Physica 34 149-54

[18] Valerini D, Creti A, Lomascolo M, Manna L, Cingolani R and Ani M 1996 Temperature dependence of the photoluminescence properties of colloidal $\mathrm{CdSe} / \mathrm{ZnS}$ 
core/shell quantum dots embedded in a polystyrene matrix Phys. Rev. B 71235409

[19] Joshi A, Narsingi K Y, Manasreh M O, Davis E A and Weaver B D 2006 Temperature dependence of the band gap of colloidal CdSe/ZnS core/shell nanocrystals embedded into an ultraviolet resin Appl. Phys. Lett. 89131907

[20] Hellwege K H and Hellege A M (ed) Landolt Bornstein: Numerical Data and Functional Relationship in Science and Technology (New Series Group III, vol 17) (Berlin: Springer)

[21] Zhang L G, Shen D, Fan Z and Lu S 2002 Exciton-phonon scattering in $\mathrm{CdSe} / \mathrm{ZnSe}$ quantum dots Chin. Phys. Lett. $19578-80$

[22] Baranov A V, Rakovich Y P, Donegan J F, Perova T S, Moore R A, Talapin D V, Rogach A L, Masumoto Y and Nabiev I 2003 Effect of ZnS shell thickness on the phonon spectra in CdSe quantum dots Phys. Rev. B 68165306

[23] Lange H, Artemyev M, Woggon U, Niermann T and Thomsen C 2008 Experimental investigation of exciton-LO-phonon coupling in CdSe/ZnS core/shell nanorods Phys. Rev. B 771933031
[24] Rubin S, Reinecke T L and Segall B 1990 Temperature-dependent exciton linewidths in semiconductors Phys. Rev. B 42 11218-31

Rubin S, Reinecke T L and Segall B 1995 Erratum: temperature-dependent exciton linewidths in semiconductors ERRATA, Phys. Rev. B 5211517

[25] Gindele F, Hild K, Langbein W and Woggon U 2000 Temperature-dependent line widths of single excitons and biexcitons J. Lumin. 87 381-3

[26] Wu Y and Arai K 1996 Temperature dependence of the photoluminescence of $\mathrm{ZnSe} / \mathrm{ZnS}$ quantum dot structures Phys. Rev. B 53 485-8

[27] Torchynska T V, Douda J and Sierra R P 2009 Photoluminescence of core-shell $\mathrm{CdSe}(\mathrm{ZnS})$ quantum dots of different sizes Phys. Status Solidi 6 S143-5

[28] Biju V, Makita Y, Sonoda A, Yokoyama H, Baba Y and Ishikawa M 2005 Temperature-sensitive photoluminescence of CdSe quantum dot clusters J. Phys. Chem. B 109 13899-905 


\title{
Spectroscopy of $\mathrm{BeAl}_{2} \mathrm{O}_{4}: \mathrm{Cr}^{3+}$ with application to high-temperature sensing
}

\author{
Devin Pugh-Thomas, ${ }^{1}$ Brian M. Walsh, ${ }^{2}$ and Mool C. Gupta ${ }^{1, \star}$ \\ 'Department of Electrical and Computer Engineering, University of Virginia, \\ Charlottesville, Virginia 22904, USA \\ ${ }^{2}$ NASA Langley Research Center, Hampton, Virginia 23681, USA \\ *Corresponding author: mgupta@ virginia.edu
}

Received 10 March 2010; revised 13 April 2010; accepted 15 April 2010; posted 15 April 2010 (Doc. ID 125250); published 17 May 2010

\begin{abstract}
Characterization of absorption, emission, and temperature-dependent luminescent features is of significant interest for the development of optical temperature sensors and photonic devices. In this work, we conduct a comprehensive study to evaluate the orientation axis-dependent absorption and emission cross sections of $\mathrm{Cr}^{3+}$ ions in $\mathrm{BeAl}_{2} \mathrm{O}_{4}$. In addition, we present new data for the temperature-dependent Starklevel energies for alexandrite. Laser-induced temperature-dependent luminescence data from 300-520 K on the $R$-line transitions are presented for application to high-temperature sensing. (C) 2010 Optical Society of America

OCIS codes: $\quad 140.5680,300.1030,300.2530,140.6810,280.6780$.
\end{abstract}

\section{Introduction}

Rare earth-doped and select transition metal iondoped optical materials are an active research area for numerous photonics applications, including fiberoptic lasers, fiber-optic amplifiers, and fiber-optic temperature sensors [1]. Solid state materials, such as phosphors, glasses, and crystals, have been used in commercial temperature measurement instruments and in various laboratory research and development temperature sensing applications. Cr-doped phosphors such as ruby $\left(\mathrm{Al}_{2} \mathrm{O}_{3}: \mathrm{Cr}^{3+}\right)$, spinel $\left(\mathrm{MgAl}_{2}\right.$ $\left.\mathrm{O}_{4}: \mathrm{Cr}^{3+}\right)$, and alexandrite $\left(\mathrm{BeAl}_{2} \mathrm{O}_{4}: \mathrm{Cr}^{3+}\right)$ have been reported for fiber-optic thermometers [2,3]. Crion-doped oxide crystals are strong candidates for temperature sensors because of their high melting temperature, visible photoluminescence, and long lifetime, on the ms order [4]. The use of alexandrite fluorescence for temperature sensing was first reported by Augusti [5] et al. It has been the subject of a temperature-sensing study using a $670 \mathrm{~nm}$ laser diode to induce alexandrite fluorescence [6]. The

0003-6935/10/152891-07\$15.00/0

(C) 2010 Optical Society of America room-temperature and low-temperature spectroscopic properties of alexandrite have been published $[7,8]$. Scalvi et al. report an increase in fluorescence intensity after natural alexandrite is subjected to a long annealing [9].

While alexandrite has been fairly well studied [5-9], the information on this material is scattered in various studies by different investigators and is incomplete in some respects. It could be said there is an absence of a comprehensive characterization in the literature. This article intends to bridge the gap between previous studies and something more comprehensive, while seeking consistency with earlier studies. Our literature search revealed no single publication on the axis-dependent fluorescence emission cross sections of alexandrite, a biaxial orthorhombic crystal. In response, we present a study of the absorption, for comparison, and emission cross sections for all axes. We also present some temperaturedependent studies in characterization. In particular, temperature-dependent emission spectra of $\mathrm{BeAl}_{2} \mathrm{O}_{4}$ : $\mathrm{Cr}^{3+}$ were acquired to assess the use of emission cross sections and the thermal line shift of the ${ }^{4} T_{1} \rightarrow{ }^{2} A_{2}$ and ${ }^{4} T_{2} \rightarrow{ }^{2} A_{2}$ transitions for thermometry. We give the first report on the temperature-dependent crystal 
field splitting of states of the $\mathrm{Cr}^{3+}$ ion in alexandrite from room temperature up to $500 \mathrm{~K}$.

\section{Experiment}

Alexandrite spectroscopic crystal samples were cut and polished from a $6.35 \mathrm{~mm} \times 12 \mathrm{~mm}$ rod with an estimated $\mathrm{Cr}^{3+}$ concentration of $0.14 \%$. Absorption spectra were acquired with a PerkinElmer Lambda 900 spectrophotometer. Absorption spectra in the wavelength range 200 to $750 \mathrm{~nm}$ were acquired at room temperature.

For continuous luminescence measurements, the excitation source used was a Spectra Physics model 2017 argon-ion laser operated at an output power of $2 \mathrm{~W}$. The luminescence is focused by an objective lens and modulated by a chopper placed at the entrance to the monochromator. The collected luminescence was focused onto a $100 \mu \mathrm{m}$ slit of a $2 \mathrm{~m}$ SPEX monochromator. A $0.10 \mathrm{~nm}$ resolution was obtained with a $100 \mu \mathrm{m}$ slit width. A 600 grooves/ mm grating blazed at $1.2 \mu \mathrm{m}$ was used in all measurements. An RG680 long-pass glass filter blocked the pump beam. A Hamamatsu R636 photomultiplier tube (PMT) detected the signal. Data were acquired using an SRS 830 lock-in amplifier coupled to a computer. Data storage and processing were accomplished using the SRS/Cd2A data acquisition computer program [10]. A mercury lamp was used to calibrate the wavelength, and a graybody light source with a color temperature of $2854 \mathrm{~K}$ was used to correct for grating efficiency, detector response, and other optical elements of the system. Temperature-dependent emission spectra of $\mathrm{BeAl}_{2} \mathrm{O}_{4}: \mathrm{Cr}^{3+}$ samples were acquired after some modification to the experimental setup. The samples were mounted in a custom-built high-temperature cell. The heating element used was a Watlow ULTRAMIC 600 ceramic heater.

Lifetime measurements were conducted using a tunable pulsed Opotek Vibrant LD 355II optical parametric oscillator. The luminescence signal was collected using an RG-695 cutoff filter, a monochromator, and a PMT. A digital oscilloscope averaged 1024 traces, and the files were saved for computer analysis. Opotek control software (V1.090) provided fully automated control of wavelength selection and pulse energy. The monochromator acted as a wavelength filter for the luminescence. The cutoff filter served to further discriminate against the pump radiation.

\section{Results and Discussion}

\section{A. Absorption Cross Sections}

The optical properties of $\mathrm{Cr}^{3+}$ in alexandrite are similar to those of $\mathrm{Cr}$ in $\mathrm{Al}_{2} \mathrm{O}_{3}$ hosts [3]. Both compounds show two well-defined $R$ lines and two broad absorption bands. However, the intensity and relative position of these transitions vary from host to host. Using a sample with a known dopant concentration $N$, absorption cross sections, $\sigma_{\mathrm{Abs}}(\lambda)$ were calculated from the transmission data using

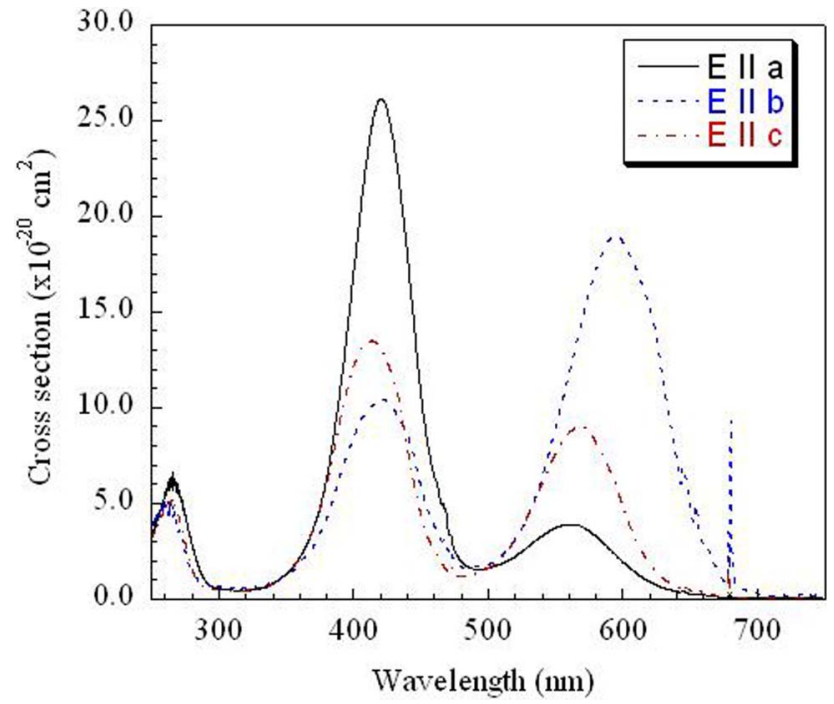

Fig. 1. (Color online) Absorption spectra of $\mathrm{BeAl}_{2} \mathrm{O}_{4}: \mathrm{Cr}^{3+}$ at $295 \mathrm{~K}$.

$$
\sigma_{\mathrm{Abs}}(\lambda)=-\frac{1}{N l} \ln \left\{\frac{[n(\lambda)+1]^{4}}{16 n^{2}(\lambda)} T\right\}
$$

where $l$ is the sample absorption path length, $T$ is the measured transmission, and $n$ is the wavelengthdependent index of refraction. The spectra were corrected for Fresnel losses at the crystal faces using the Sellimeier's coefficients for alexandrite [11]. The absorption spectra for three polarizations of light are reported in Fig. 1. The absorption spectra consists of two broad bands centered at 420 and $580 \mathrm{~nm}$ along the $b$ axis, and 420,560,400, and $563 \mathrm{~nm}$ along the $a$ and $c$ axes, respectively. An absorption band edge peak appears at $260 \mathrm{~nm}$. The absorption data are in agreement with reports made by other authors [7-9]. Table 1 summarizes the peak absorption cross section values. Our results show best agreement with the Northrop data for $\mathbf{E} \| \mathbf{b}$ [12]. However, no $\mathbf{E} \| \mathbf{a}$ or $\mathbf{E} \| \mathbf{c}$ data are available for comparison. The Walling et al. data [7] are consistently lower by about $20 \%$. The Watanabe et al. data [13] are consistently higher by about $20 \%$, on average. This is reasonable, considering the uncertainty inherent in absorption cross section measurements, where the $\mathrm{Cr}$ concentration is

Table 1. Comparison of Peak Absorption Cross Sections $\left(\times 10^{-19} \mathrm{~cm}^{2}\right)$ of $\mathrm{R}$ Lines in $\mathrm{BeAl}_{2} \mathrm{O}_{4}: \mathrm{Cr}^{3+}$

\begin{tabular}{ccccc}
\hline Transition & $\begin{array}{c}\text { This } \\
\text { Study }\end{array}$ & $\begin{array}{c}\text { Walling } \\
\left.\text { et al. }[]^{a}\right]^{a}\end{array}$ & $\begin{array}{c}\text { Northrop } \\
{[12]}\end{array}$ & $\begin{array}{c}\text { Watanabe } \\
\text { et al. [13] }\end{array}$ \\
\hline $\mathbf{E} \| \mathbf{a}$ & & & - & 3.5 \\
${ }^{2} A_{2} \rightarrow{ }^{4} T_{1}$ & 2.6 & 1.9 & - & 0.4 \\
${ }^{2} A_{2} \rightarrow{ }^{4} T_{2}$ & 0.39 & 0.32 & 1.05 & 1.25 \\
$\mathbf{E} \| \mathbf{b}$ & & & 1.78 & 2.15 \\
${ }^{2} A_{2} \rightarrow{ }^{4} T_{1}$ & 1.04 & 0.77 & & 2.2 \\
${ }^{2} A_{2} \rightarrow{ }^{4} T_{2}$ & 1.9 & 1.4 & - & 1.2 \\
$\mathbf{E} \| \mathbf{c}$ & & & - & \\
${ }^{2} A_{2} \rightarrow{ }^{4} T_{1}$ & 1.35 & 1.27 & 0.68 & \\
${ }^{2} A_{2} \rightarrow{ }^{4} T_{2}$ & 0.9 & & & \\
\hline
\end{tabular}

${ }^{a}$ Derived from Fig. 3 in this reference. 
not always exactly known. We validate our $\mathrm{Cr}$ concentration of $\sim 0.14 \%$ through reciprocity of absorption and emission using McCumber's theory, giving confidence to our measurements presented in this article.

\section{B. Emission Cross Sections}

In the 1960s, D. E. McCumber published a paper in which he derived generalizations of Einstein's concepts of stimulated emission and absorption for impurity centers in insulating solids [14]. McCumber, along the lines of Einstein's theory, showed that emission and absorption are reciprocal, and theore-
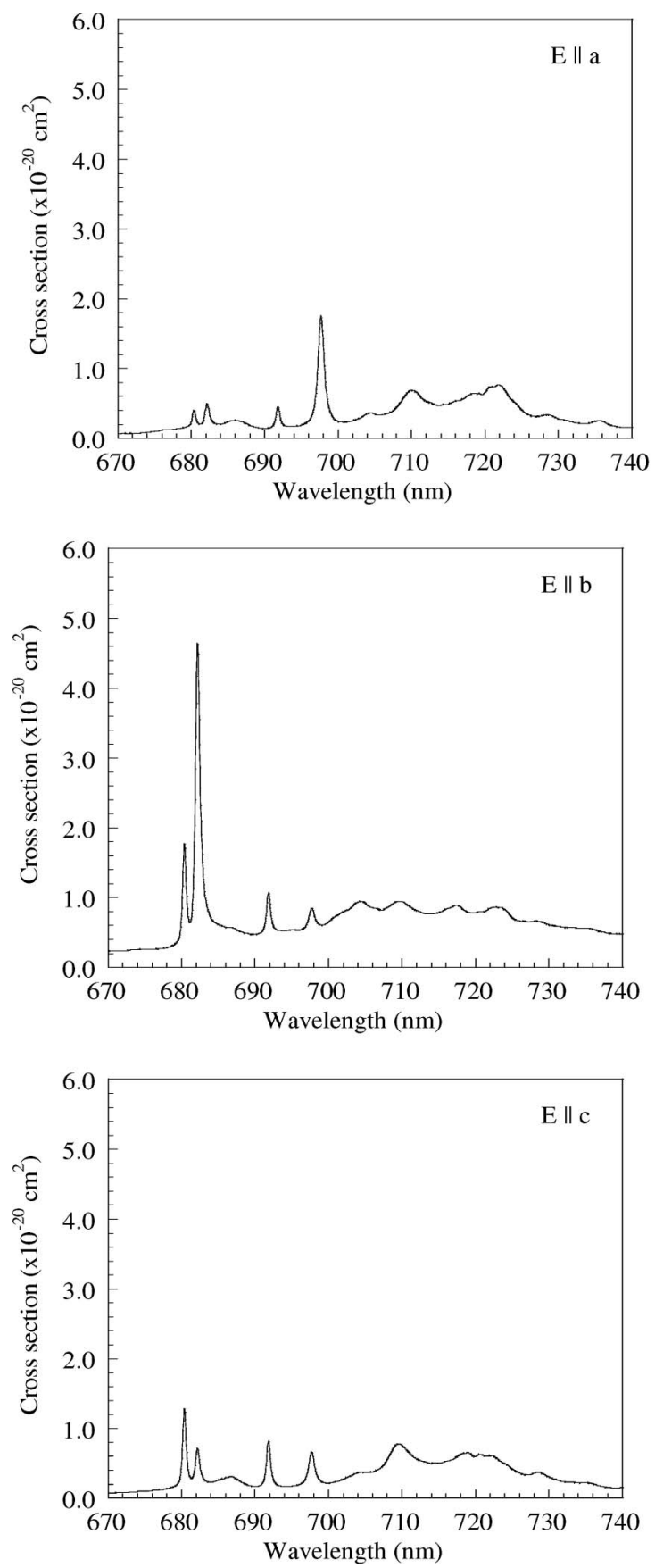

tically related in quantifiable terms. The ideas of McCumber are often useful in spectroscopic studies to validate measurements in absorption and emission studies, and to test Judd-Ofelt theory [15]. The emission cross section can be written as

$$
\sigma_{\mathrm{em}}(\lambda)=\frac{\lambda^{5}}{8 \pi c n^{2}\left(\tau_{r} / \beta\right)} \frac{I(\lambda)}{\int \lambda I(\lambda) \mathrm{d} \lambda}
$$

where $I(\lambda)$ is the measured emission intensity at a wavelength $\lambda, \tau_{r}$ is the radiative lifetime, $\beta$ is the branching ratio, $n$ denotes the index of refraction,
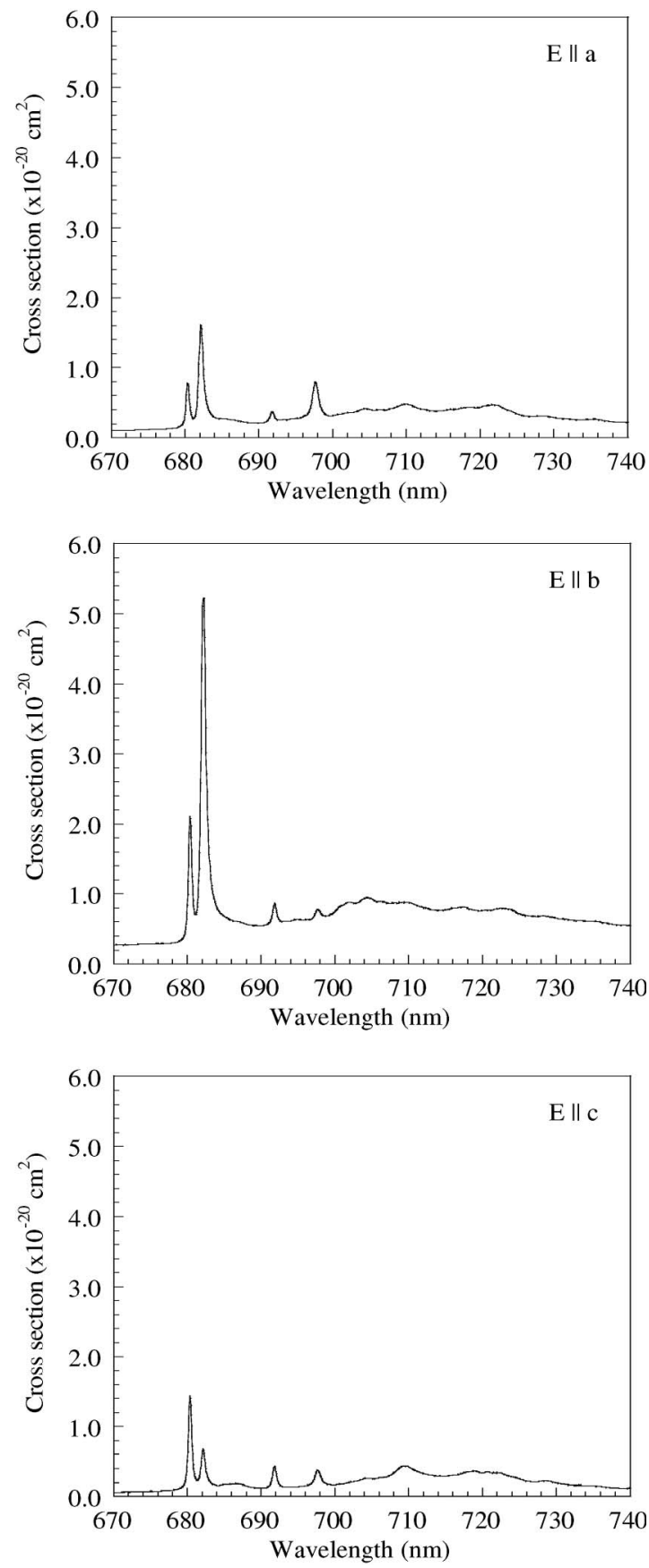

Fig. 2. Alexandrite emission cross section: $\mathbf{E} \| \mathbf{a}$ (top row), $\mathbf{E} \| \mathbf{b}$ (middle row), $\mathbf{E} \| \mathbf{c}$ (bottom row), pump wavelength $=488 \mathrm{~nm}$ (left column), pump wavelength $=514 \mathrm{~nm}$ (right column), and temperature $=295 \mathrm{~K}$. 

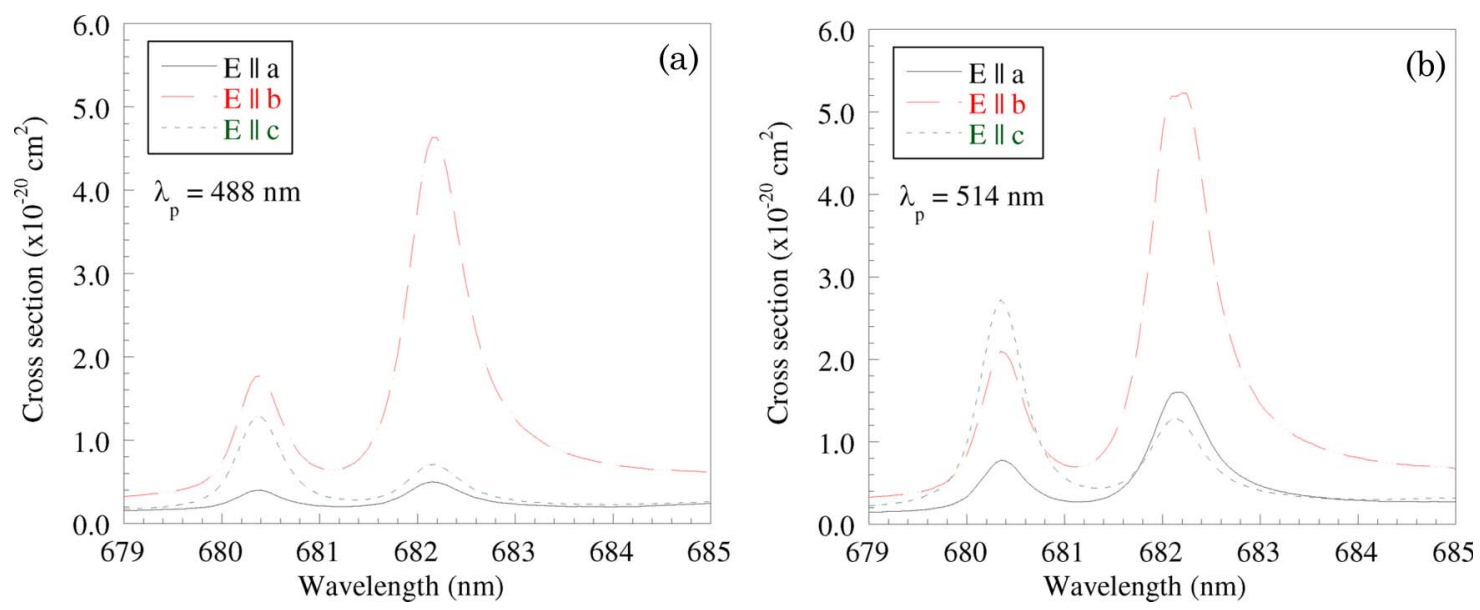

Fig. 3. (Color online) Emission cross sections of alexandrite $R$ lines at $T=295 \mathrm{~K}$ for (a) $488 \mathrm{~nm}$ and (b) $514.5 \mathrm{~nm}$.

and $c$ is the speed of light. Equation (2) can be extended to biaxial crystals for the electric field of emission polarized parallel to the $a, b$, and $c$ axes in the following way:

$$
\sigma_{\mathrm{em}}(\lambda)=\frac{\lambda^{5}}{8 \pi c n^{2}\left(\tau_{r} / \beta\right)} \frac{I_{a}(\lambda)}{\int\left[\frac{1}{3} I_{a}(\lambda)+\frac{1}{3} I_{b}(\lambda)+\frac{1}{3} I_{c}(\lambda)\right] \lambda \mathrm{d} \lambda} .
$$

The theory of McCumber has been discussed in many articles. Some exploration of the McCumber theory can be found in the articles of Walsh et al. [15], Payne et al. [16], and Miniscalco and Quimbly [17]. In fundamental ways, based on McCumber's work, the reciprocity of absorption and emission can be expressed as [15-17]

$$
\sigma_{\mathrm{em}}(\lambda)=\sigma_{\mathrm{abs}}(\lambda) \frac{Z_{l}}{Z_{u}} \exp \left[\frac{E_{\mathrm{ZL}}-h c / \lambda}{k T}\right]
$$

where $Z_{1} / Z_{u}$ is the ratio of partition functions of the lower and upper manifolds and $E_{\mathrm{ZL}}$ is the zero line representing the difference in energy $\left(\mathrm{cm}^{-1}\right)$ from the lowest level of the upper manifold to the lowest level of the lower manifold. Boltzmann's constant is $k$, Planck's constant is $h$, and $T$ is the temperature.

The measured room-temperature fluorescence spectra in the wavelength range $670-740 \mathrm{~nm}$ are shown in Fig. 2. for excitation wavelengths at 488 and $514.5 \mathrm{~nm}$. A lifetime $\tau=260 \mu \mathrm{s}[7,18]$ at room temperature was used for calculating the cross section in Eq. (3). The calculations are supported by a measured radiative quantum efficiency of $0.95 \pm$ 0.05 for alexandrite [19]. A room-temperature lifetime, $\tau=260 \mu \mathrm{s}$ is the same as is measured in this study. Temperature-dependent lifetime will be presented later in Subsection 3.C. The fluorescence spectrum of $\mathrm{BeAl}_{2} \mathrm{O}_{4}: \mathrm{Cr}^{3+}$ consists of two sharp $R$ lines superimposed on a broad vibronic emission background with a sideband extending out to $750 \mathrm{~nm}$.
The $R$ line transitions from the excited states ${ }^{4} T_{1}$ and ${ }^{4} T_{2}$ to the ground state ${ }^{2} A_{2}$ at room temperature are shown in Fig. 3. This figure presents the same data as Fig. 2, but just shows the $R$ lines only in an expanded view.

Our analysis of the emission cross section here is done for comparison with the results of Shand and Jenssen [18]. Comparing their Fig. 1 with our results here, we find an order of magnitude agreement, and it is within a factor of 2 . While a discrepancy of $\pm 20 \%$ might be expected for experimental uncertainty, a factor of 2 in difference cannot be entirely explained. It is unclear from the work of Shand and Jenssen if they corrected their emission spectra for system response with a calibrated standard lamp, as the details of their experiment are not presented in this regard. The orientation of the crystal for polarization studies could also be a factor. We found that, due to the large difference in emission yield from the $b$ axis compared to the $a$ and $c$ axis, alignment of the crystal with respect to the pump source as well as orientation of the polarizer

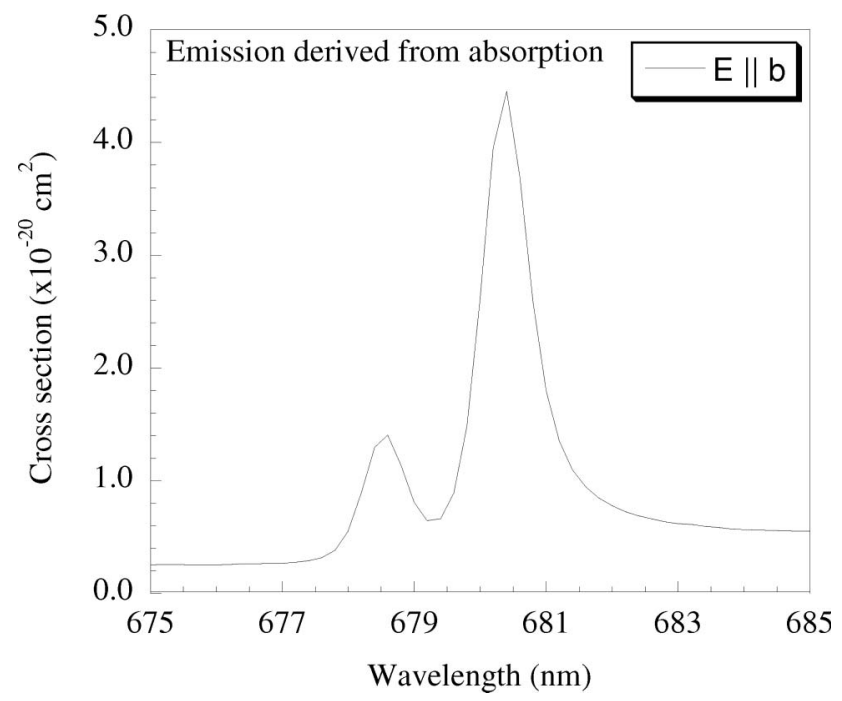

Fig. 4. Derived emission cross section for $\mathbf{E} \| \mathbf{b}$ polarization in $\mathrm{BeAl}_{2} \mathrm{O}_{4}: \mathrm{Cr}^{3+}$. 
for fluorescence selection is sensitive and care must be exhibited. In our studies, we sought reproducibility in our experiments. In addition, different pump wavelengths were utilized. While there is some additional structure in the long wavelength sideband at different pump wavelengths, the $R$ lines had approximately the same cross sections in all cases. In addition to the 488 and $514 \mathrm{~nm}$ pump wavelengths, we also conducted studies at a $632 \mathrm{~nm}$ pump wavelength. While the results of the $632 \mathrm{~nm}$ studies are not presented here, the cross sections are approximately the same as the other pump wavelength studies presented here. It is noted here that the longer the pump wavelength, the less structure there appeared to be in the vibronic sideband out to $750 \mathrm{~nm}$. Nevertheless, the $R$ lines demonstrated similar cross sections, regardless of the pump wavelength. Within our study, the results are self-consistent.

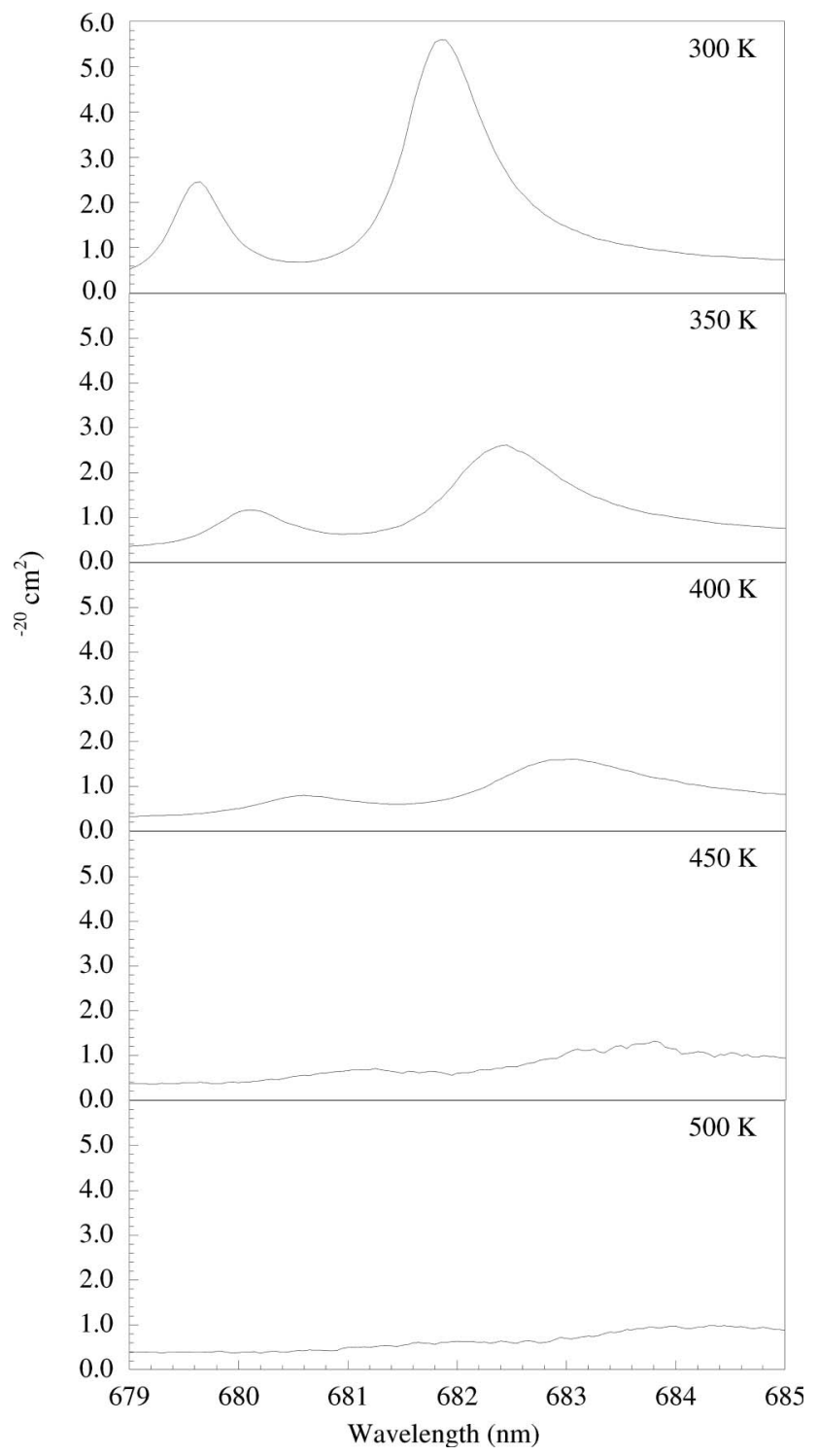

Fig. 5. Emission cross section versus temperature of $\mathrm{BeAl}_{2} \mathrm{O}_{4}: \mathrm{Cr}^{3+}$ for $\mathbf{E} \| \mathbf{b}$ polarization.

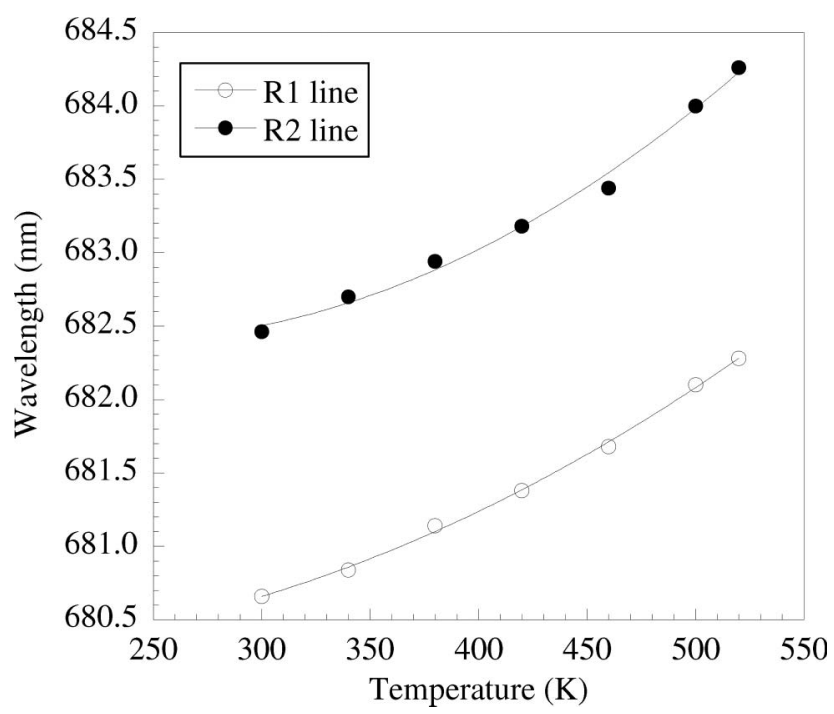

Fig. 6. Peak wavelength versus temperature of emission lines in $\mathrm{BeAl}_{2} \mathrm{O}_{4}: \mathrm{Cr}^{3+}$ for $\mathbf{E} \| \mathbf{b}$ polarization.

It is useful to determine the emission cross-section spectrum as derived from absorption measurements using the McCumber theory. This provides a check on the absorption and emission measurements, as mentioned previously. The derived emission cross-section spectrum for $\mathbf{E} \| \mathbf{b}$ in $\mathrm{BeAl}_{2} \mathrm{O}_{3}: \mathrm{Cr}^{3+}$ is shown in Fig. 4 . The agreement is reasonably good with the measured emission in Fig. $\underline{3}$, noting the Stokes shift in wavelength of $\sim 2 \mathrm{~nm}$.

\section{Temperature-Dependent Fluorescence}

Temperature changes affect the observed transition wavelengths and the relative occupation probabilities within the sublevels. The latter effect is often more pronounced. The lower the temperature, the more the level populations are concentrated on the lowest-lying sublevels. Higher temperatures allow for absorption at longer wavelengths (starting from

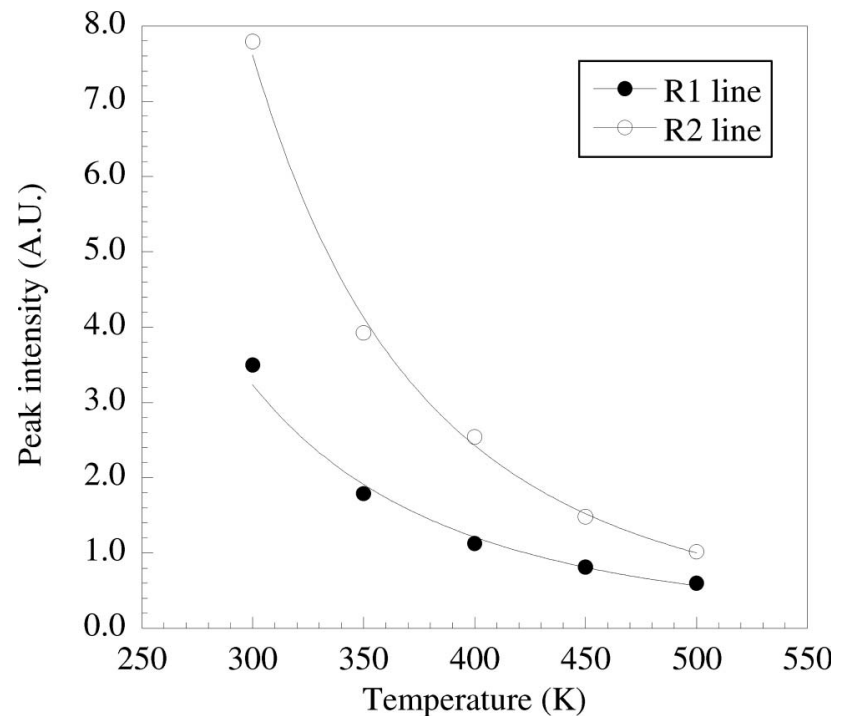

Fig. 7. Peak intensity versus temperature of emission lines in $\mathrm{BeAl}_{2} \mathrm{O}_{4}: \mathrm{Cr}^{3+}$ for $\mathbf{E} \| \mathbf{b}$ polarization. 
Table 2. Stark-Level Energy $\left(\mathrm{cm}^{-1}\right)$ of $\mathrm{BeAl}_{2} \mathrm{O}_{4}: \mathrm{Cr}^{3+}$ for $\mathrm{E} \| \mathrm{b}$ Polarization

\begin{tabular}{cccccccc}
\hline Multiplet & Degeneracy & Transition & $300 \mathrm{~K}$ & $350 \mathrm{~K}$ & $400 \mathrm{~K}$ & $450 \mathrm{~K}$ \\
\hline${ }^{2} E$ & 2 & $R_{1}$ & 14,695 & 14,687 & 14678 & 14668 \\
& 2 & $R_{2}$ & 14,734 & 14,726 & 14719 & 14710 \\
${ }^{2} T_{1}$ & 2 & $T_{1}$ & 15,506 & 15,497 & 15486 & 15477 \\
& 2 & $T_{2}$ & 15,273 & 15,266 & 15259 & 15248 & 15472 \\
& 2 & $T_{3}$ & 15,076 & 15,076 & 15076 & 15053 & 15048 \\
\hline
\end{tabular}

higher-lying sublevels of the lower manifold) and for emission at shorter wavelengths (starting from higher-lying sublevels of the upper manifold). In alexandrite, higher temperatures tend to increase the absorption and decrease emission from $675-685 \mathrm{~nm}$, and as a consequence, reduced emission of the nophonon $R$-line peaks is observed. At high temperatures, excitations can spread over a large number of levels in the excited vibronic states. Radiative transitions can take place between any set of upper and lower vibronic states, but phonons and photons are competing. In such situations, a broadening and shift of the $R$ lines is expected and indeed observed in Fig. 5. The variation in peak wavelength with temperature is reported in Fig. 6 . The variation of intensity with temperature of the $R$ lines is shown in Fig. 7. Table 2 reports the Stark-level energy variation of transitions from 300-500 K. Our literature search found Stark-level energy data at 300 and $375 \mathrm{~K}$ [7]. To the best of our knowledge, this study is the first series of measurements reporting on Stark-level energies at elevated temperatures above $375 \mathrm{~K}$. Measurements here are presented up to $500 \mathrm{~K}$ for the energy levels and emission cross sections.

Alexandrite is an interesting material, and this is especially true of its temperature-dependent properties. Our studies here reveal that the spectroscopic properties are highly temperature dependent. It would be interesting to see some studies of this material extending above $500 \mathrm{~K}$, which is beyond the

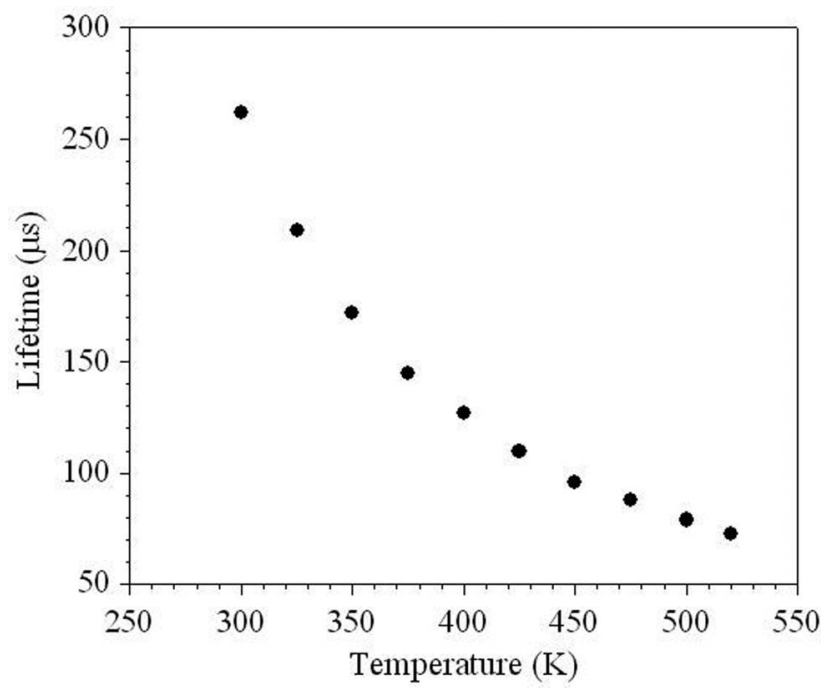

Fig. 8. Temperature-dependent lifetime of $\mathrm{BeAl}_{2} \mathrm{O}_{4}: \mathrm{Cr}^{3+}$. scope of this article. Our extension to $500 \mathrm{~K}$ has revealed some interesting aspects that warrant further study at even higher temperatures.

In Fig. 8 we present the temperature-dependent lifetime of alexandrite. The lifetime results presented here are in agreement with previous studies [6-8] and support a fluorescent lifetime of $\sim 260 \mu$ s at room temperature, which was used to calculate emission cross sections in this work. The elevated temperature lifetime tracks well with the references given here.

Thermal effects can be exploited for thermometry, as reported by several authors for different materials. One such effect, the line shift of alexandrite $R$ lines exhibits a temperature dependence. The thermal line shift, SE can be approximated by the following equation [20]:

$$
\mathrm{SE}\left(\mathrm{cm}^{-1}\right)=\alpha\left(\frac{T}{T_{D}}\right)^{4} \int_{0}^{T_{D} / T} \frac{x^{3}}{e^{x}-1} \mathrm{~d} x
$$

where $\alpha$ is the coupling coefficient for the electronphonon interaction and $T_{D}$ is the Debye temperature of the crystal. The line shift due to thermal expansion of the host lattice is negligible compared to the shift associated with the electron-phonon interaction. Equation (5) was used to fit experimental data, taking $T_{D}$ and $\alpha$ as adjustable parameters. The solid line in Fig. 9 is calculated to the best fit with $T_{D}=440 \mathrm{~K}$ and $\alpha=200 \mathrm{~cm}^{-1}$ for both $R$ lines. The Debye

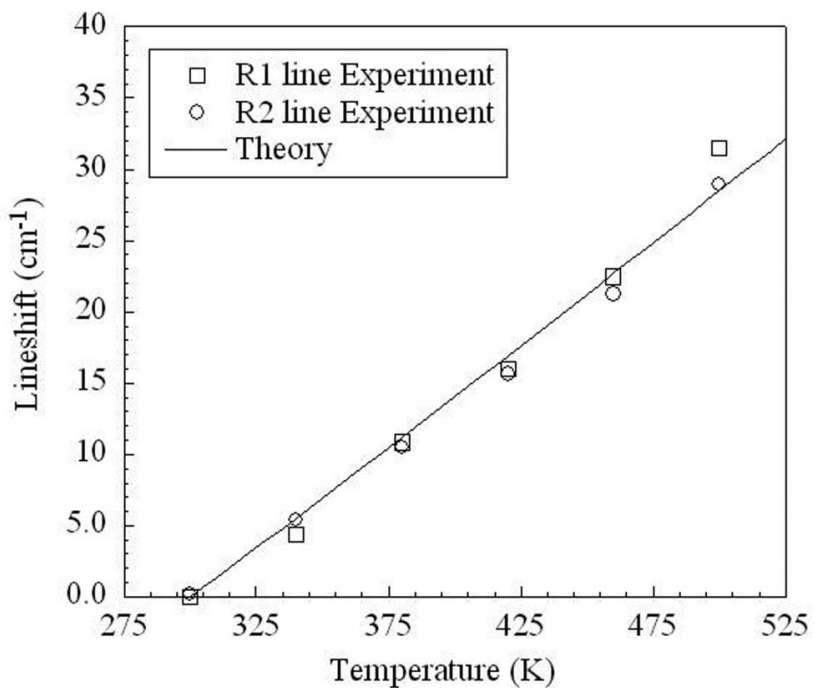

Fig. 9. Thermal line shift of $R$ lines versus temperature with respect to its position at $300 \mathrm{~K}$ for $\mathbf{E} \| \mathbf{b}$ polarization. 
temperature revealed here is in approximate agreement with the estimate of Powell et al. [8] of $T_{D} \sim 500 \mathrm{~K}$.

\section{Summary}

The room-temperature absorption and laser-induced emission features of alexandrite are reported. Temperature-dependent luminescent emission from $\mathrm{BeAl}_{2} \mathrm{O}_{4}: \mathrm{Cr}^{3+} R$ lines have been measured for application to optical temperature sensing. The absorption and emission cross sections associated with the $\mathrm{Cr}^{3+}$ ion transitions have been determined, and by applying the McCumber theory, reciprocity is shown. Temperature-dependent emission spectra of $\mathrm{BeAl}_{2} \mathrm{O}_{4}: \mathrm{Cr}^{3+}$, axis orientation-dependent luminescence, and the thermal line shift are applicable for temperature sensing and can be exploited for optical temperature sensors. The line shift is used here as a demonstration. New data for the $\mathrm{BeAl}_{2} \mathrm{O}_{4}: \mathrm{Cr}^{3+}$ Stark-energy levels above $300 \mathrm{~K}$ are provided. New data on the calculated $R$-line thermal line shift versus experimental data on alexandrite are reported. We show good agreement between the theory and experimental data, demonstrating that the line shift can be used as a potential thermal sensor. This can be compared to other methods [21].

The authors thank William S. Luck and William C. Edwards for their support. We also acknowledge fellowship support from the NASA Graduate Student Researchers program. We thank NASA/National Institute of Aerospace (NIA) for support through the Langley Professor program.

\section{References}

1. A. Khalid and K. Kontis, "Thermographic phosphors for high temperature measurements: principles, current state of the art and recent applications," Sensors 8, 5673-5744 (2008).

2. M. McSherry, C. Fitzpatrick, and E. Lewis, "Review of luminescent based fiber optic temperature sensors," Sensor Rev. 25, 56-62 (2005).

3. H. C. Seat, J. H. Sharp, Z. Y. Zhang, and K. T. V. Grattan, "Single-crystal ruby fiber temperature sensor," Sens. Actuators A, Phys. 101, 24-29 (2002).

4. H. Aizawa, H. Uchiyama, T. Katsumata, S. Komuro, T. Morikawa, H. Ishizawa, and E. Toba, "Fiber-optic thermometer using sensor materials with long fluorescence lifetime," Meas. Sci. Technol. 15, 1484-1489 (2004).
5. A. T. Augousti, K. T. V. Grattan, and A. W. Palmer, "A laserpumped temperature sensor using the fluorescent decay time of alexandrite," J. Lightwave Technol. 5, 759-762 (1987).

6. Z. Zhang, G. T. V. Grattan, and A. W. Palmer, "Fiber-optic high-temperature sensor based on the fluorescence lifetime of alexandrite," Rev. Sci. Instrum. 63, 3869-3873 (1992).

7. J. C. Walling, O. G. Peterson, H. P. Jenssen, and R. C. Morris, “Tunable alexandrite lasers," IEEE J. Quant. Electron. 16, 1302-1314 (1980).

8. R. C. Powell, L. Xi, X. Gang, and G. J. Quarles, "Spectroscopic properties of alexandrite crystals," Phys. Rev. B 32, 27882797 (1985).

9. R. M. Scalvi, M. S. Li, and L. V. A. Scalvi, "Annealing effects on optical properties of natural alexandrite," J. Phys. Condens. Matter 15, 7437-7443 (2003).

10. SRS/Cd2A program written by coauthor B. M. Walsh.

11. N. P. Barnes, "Solid-state lasers from an efficiency perspective," IEEE J. Quant. Electron. 13, 435-447 (2007).

12. http://www.as.northropgrumman.com/products .synoptics_alexandrite/assets/Alexandrite.

13. S. Watanabe, T. Sasaki, R. Taniguchi, T. Ishii, and K. Ogasawara, "First principles calculation of ground and excited-state absorption spectra of ruby and alexandrite considering lattice relaxation," Phys. Rev. B. 79, 075109 (2009).

14. D. E. McCumber, "Einstein relations connecting broadband emission and absorption spectra," Phys. Rev 136, A954-A957 (1964).

15. B. M. Walsh, N. P. Barnes, and B. Di Bartolo, "Branching ratios, cross sections, and radiative lifetimes of rare earth ions in solids; application to $\mathrm{Tm}^{3+}$ and $\mathrm{Ho}^{3+}$ ions in $\mathrm{LiYF}_{4}$," J. Appl. Phys. 83, 2772-2787 (1998).

16. S. A. Payne, L. L. Chase, L. K. Smith, W. L. Kray, and W. P. Krupke, "Infrared cross section measurements for crystals doped with $\mathrm{Er}^{3+}, \mathrm{Tm}^{3+}$, and $\mathrm{Ho}^{3+}$," IEEE J. Quant. Electron. 28, 2619-2630 (1992).

17. W. J. Miniscalco and R. S. Quimbly, "General procedure for the analysis of $\mathrm{Er}^{3+}$ cross sections," Opt. Lett. 16, 258-260 (1991).

18. M. L. Shand and H. P. Jenssen, "Temperature dependence of the excited-state absorption of alexandrite," IEEE J. Quant. Electron. 19, 480-484 (1983).

19. M. L. Shand, "Quantum efficiency of alexandrite," J. Appl. Phys. 54, 2602-2604 (1983).

20. B. Di Bartolo, Optical Interactions in Solids (Wiley, 1968).

21. T. Sun, Z. Y. Yang, K. Y. V. Grattan, and A. W. Palmer, "Alexandrite-based optical temperature sensing: comparison of different fluorescence-based approaches," in Proceedings of the 12th International Conference on Optical Fiber Sensors (Optical Society of America, 1997), Vol. 16. 


\section{DEVIN PUGH-THOMAS}

\section{Education}

Doctor of Philosophy (Electrical Engineering)

University of Virginia, Charlottesville, VA

May 2012

Master of Science (Materials Science and Engineering)

January 2003

University of Virginia, Charlottesville, VA

Bachelor of Science (Physics)

May 2000

Norfolk State University, Norfolk, VA

\section{Experience}

NASA Langley Research Center, Hampton, VA

Electrical Engineer, Graduate Co-op

4/2010- Present

University of Virginia-National Institute of Aerospace

8/ 2007-3/2010

Hampton, VA

Graduate Research Assistant

Norfolk State University, Norfolk, VA

$5 / 2003-8 / 2007$

Instructor

University of Virginia, Charlottesville, VA

$8 / 2000-12 / 2002$

Graduate Research Assistant

NASA Goddard Space Flight Center, Greenbelt, MD

$6 / 2000-8 / 2000$

Electrical Engineer, Intern

NASA Langley Research Center, Hampton, VA

6/1998-8/1999

Optical Engineer, Intern 


\section{$\underline{\text { Honors and Awards }}$}

2011

2007

1998

1998
Incubic/Milton Chang Travel Grant Award Recipient

Outstanding University Service Award

Golden Key International Honour Society, Lifetime Member

Alpha Kappa Mu National Honor Society

Fellowships

2008 NASA Graduate Student Researchers Program (GSRP) Fellow

$2007 \quad$ National Institute of Aerospace (NIA) Fellow

2000 National Science Foundation Integrative Graduate Education and Research Traineeship (NSF-IGERT) Fellow

1996 Dozoretz National Institute for Mathematics and Applied Sciences (DNIMAS) Scholar

\section{Grantsmanship}

“Tailor-Made: Quantum Dots for Remote Sensing," Co-Principal Investigator, NASA Langley Research Center Creativity and Innovation (C\&I) Fund, Awarded Funding: 10K, March 2011.

"Students in Technology Enhanced Physical Science (STEPS): From Passive to Active Learning," Principle Investigator, Norfolk State University College of Science, Engineering, and Technology, Awarded Funding: 5K, September 2006.

\section{$\underline{\text { Presentations }}$}

Invited Speaker, "Quantum Dots for Luminescence Thermometry and Remote Sensing," Nondestructive Evaluation Sciences Branch, NASA Langley Research Center, Hampton, VA, January 2012

"Quantum Dots for Luminescence Thermometry and Remote Sensing," Graduate Student Review, National Institute of Aerospace, Hampton, VA, October 2011.

Keynote Speaker, NASA OSSI Regional Awareness Day, North Carolina A\&T State University, Greensboro, NC, February 2011.

"Luminescent Quantum Dots for High Temperature Sensing," Graduate Student Review, National Institute of Aerospace, Hampton, VA, January 2011. 
Invited Speaker, "High Temperature Fluorescence Sensor," NIA Technical Advisory Committee Meeting, National Institute of Aerospace, Hampton, VA, May 2010.

Invited Speaker, “High Temperature Fluorescence Sensor," Laser Remote Sensing Branch Technical Forum, NASA Langley Research Center, Hampton, VA, May 2010.

"High Temperature Fluorescence Sensor,” NASA Graduate Student Researchers Program Orientation, NASA Langley Research Center, Hampton, VA, July 2009.

Invited Speaker, "High Temperature Fluorescence Sensor," American Institute of Aeronautics and Astronautics (AIAA) Meeting, National Institute of Aerospace, Hampton, VA, April 2009.

Invited Speaker, "Characterization of Nanoscale Porosity and Cracking in Amorphous Solid Water Ice,” Norfolk State University, Norfolk, VA, November 2003.

Best Poster Award, National Science Foundation Materials Research Science and Engineering Center (NSF-MRSEC) Annual Conference, Charlottesville, VA, November 2002.

Best Poster Award, Graduate Student Poster Contest, Center for Innovative Technology, Herndon, VA, June 2001.

Best Oral Presentation, "High Resolution Rayleigh Scattering," Summer Intern Technical Presentations, NASA Langley Research Center, Hampton, VA, August 1998.

Invited Speaker, "Michelin Tire Project," Summer Internship Closing Ceremony, NASA Langley Research Center, Reid Conference Center, Hampton, VA, August 1995.

\section{Conferences}

Conference on Lasers and Electro-Optics (CLEO), Speaker, "Quantum Dots for High Temperature Sensing,” Baltimore Convention Center, Baltimore, MD, May 2011.

$5^{\text {th }}$ Annual Aerospace Days in the State Capitol, Panelist, "The NASA Pipeline: Students in STEM Disciplines,” State Capitol Building, Richmond, VA, February 2010.

Future Faculty: Diverse Leaders of Tomorrow Workshop, Chalk-talk Presenter, "Quantum Dots for Thermal Sensing," Carnegie Mellon University, Pittsburgh, PA, August 2009. 


\section{$\underline{\text { Professional Affiliations }}$}

2010- Present Optical Society of America (OSA) Member

2012- Present Institute of Electrical and Electronics Engineers (IEEE) Member

\section{$\underline{\text { Media Appearances }}$}

NASA Langley Research Center “Center Snapshot: Devin Pugh-Thomas”, Researcher News, http://www.nasa.gov/centers/langley/news/researchernews/snapshot_pugh-thomas.html , February 2010.

NASA Langley Research Center, “The Langley Story” Video, June 2010.

National Institute of Aerospace, Graduate Education Student Promotional Video, http://www.nianet.org/resources/Education/Graduate-Education/Graduate-Program.aspx , June 2009. 